\title{
(1)
}

UNIVERSIDAD PERUANA DE CIENCIAS APLICADAS ESCUELA DE POSTGRADO

PROGRAMA DE MAESTRÍA EN DIRECCIÓN DE LA CONSTRUCCIÓN

\section{PROPUESTA DE IMPLEMENTACIÓN DE MEJORAMIENTO PARA REDUCIR EL TRABAJO NO CONTRIBUTORIO. CASO DE ESTUDIO: VIVIENDA MULTIFAMILIAR “TWENTY” EN EL DISTRITO DE LINCE- LIMA}

\section{TRABAJO DE INVESTIGACIÓN}

Para optar el grado académico de Maestro en Dirección de la Construcción

\section{AUTORES}

Hinostroza Capani, José Cristhian (0000-0002-4857-9192)

Jurado Tasayco, Sebastian (0000-0003-0205-7660)

Manrique Tarazona, María Katty (0000-0002-6827-795X)

\section{ASESOR DE TRABAJO DE INVESTIGACIÓN}

Uehara Yagi, José Daniel (0000-0002-9190-6443)

Lima, 30 de octubre de 2019 


\section{DEDICATORIA}

A nuestros padres, por su amor, trabajo y sacrificio en todos estos años, gracias a ustedes hemos logrado llegar hasta aquí y convertirnos en lo que somos. 


\section{AGRADECIMIENTOS}

Agradecemos a nuestros docentes de la Escuela de Postgrado de la Universidad Peruana de Ciencias Aplicada (UPC), por haber compartido sus conocimientos a lo largo de la preparación de nuestra profesión, de manera especial, al ingeniero José Daniel Uehara Yagi asesor de nuestro trabajo de investigación quien ha guiado con su paciencia, y su rectitud como docente. 


\section{RESUMEN}

El presente trabajo de investigación realiza el análisis, evaluación y mejoramiento de la productividad en el caso de estudio: Edificio multifamiliar Twenty - Lince. Mediante la implementación y uso de herramientas Last Planner en la ejecución de la obra en mención.

Los indicadores de productividad mostrados en este trabajo de investigación son: Trabajo productivo, trabajo contributorio y trabajo no contributorio, los cuales se miden con ayuda de la herramienta carta balance. Esto con el objetivo de ser mejorados con la implementación de procesos basados en la metodología Last Planner.

En el primer capítulo se plantea la problemática, se establece el objetivo general y los objetivos específicos, así como la justificación, metodología y límites de la misma. El segundo capítulo refiere al marco teórico. En el tercer capítulo se expone la metodología usada y el levantamiento de información en el caso de estudio, esto se refiere a la toma de datos en la ejecución de la obra con el uso de la carta balance. En el cuarto capítulo se muestra los resultados de la implementación de las mejoras de solución propuestas en este mismo capítulo. La identificación y liberación de restricciones, también se aborda como tema central en este capítulo. En el quinto y último capítulo se presentan las conclusiones y recomendaciones a las que se llegaron en este trabajo de investigación. Se responde al objetivo general y objetivos específicos de la presente; y se finaliza con las recomendaciones para el aumento de la productividad en las obras.

Palabras clave: Last Planner; Trabajo productivo; Trabajo contributorio; Trabajo no contributorio; Productividad. 


\title{
IMPLEMENTATION PROPOSAL FOR IMPROVEMENT TO REDUCE NON- CONTRIBUTORY WORK. CASE STUDY: “TWENTY” MULTIFAMILIAR HOUSING IN THE DISTRICT OF LINCE - LIMA
}

\begin{abstract}
This research work carries out the analysis, evaluation and improvement of productivity in the case study: Twenty - Lince multifamily building. Through the implementation and use of Last Planner tools in the execution of the work in question.

The productivity indicators shown in this research work are: Productive work, contributory work and non-contributory work, which are measured with the help of the balance sheet tool. This with the objective of being improved with the implementation of processes based on the Last Planner methodology.

In the first chapter the problem is raised, the general objective and the specific objectives are established, as well as the justification, methodology and limits of the same. The second chapter refers to the theoretical framework. In the third chapter, the methodology used and the gathering of information in the case study is presented, this refers to the collection of data in the execution of the work with the use of the balance sheet. The fourth chapter shows the results of the implementation of the solution improvements proposed in this chapter. The identification and release of restrictions is also addressed as a central issue in this chapter. The conclusions and recommendations reached in this research paper are presented in the fifth and final chapter. It responds to the general objective and specific objectives of the present; and ends with recommendations for increasing productivity in the works.
\end{abstract}

Keywords: Last Planner; Productive work; Contributory work; Non-contributory work; Productivity. 


\section{TABLA DE CONTENIDOS}

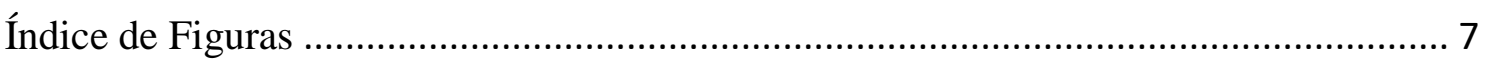

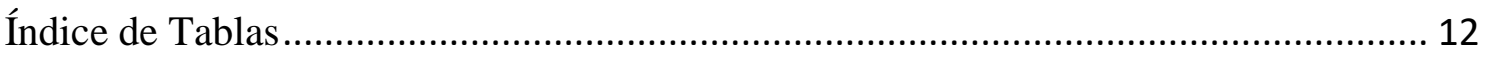

Índice de Fotografías ............................................................................................ 14

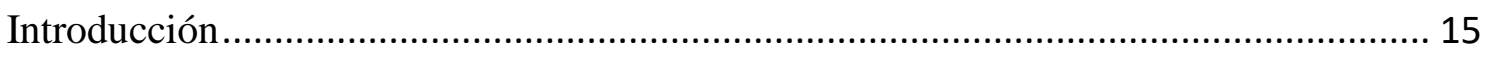

Capítulo I: Aspectos Generales ......................................................................... 16

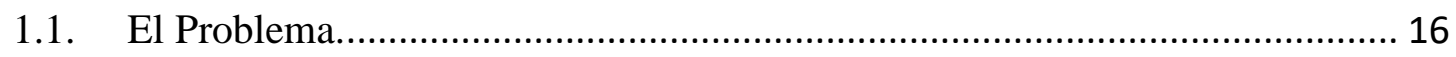

1.2. Formulación del Problema.............................................................................. 17

1.3. Objetivo General.................................................................................... 18

1.4. Objetivo Específicos.............................................................................. 18

1.5. Justificación del Problema........................................................................... 18

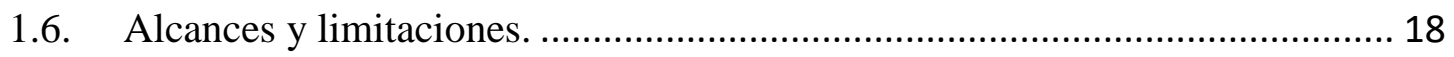

1.7. Metodología de Investigación................................................................... 19

Capítulo II: Marco Teórico ...................................................................................... 20

2.1. Productividad en la Construcción.................................................................... 20

2.2. Categorías de Trabajo en la Construcción. ...................................................... 26

2.2.1. Trabajo No Contributorio: Investigaciones y Causas................................. 28

2.2.2. Herramienta de medición de Trabajo: Carta Balance................................. 37

2.3. Estrategias para mejorar la Productividad..................................................... 38

2.3.1. Ciclo de Mejoramiento de la Productividad ................................................. 39

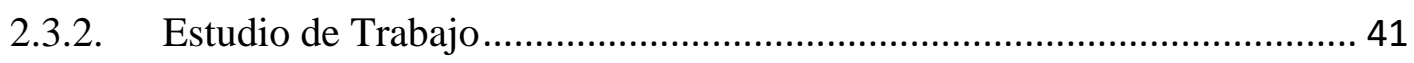

2.3.3. Last Planner System .............................................................................. 43

Capítulo III: Metodología y Levantamiento de Información .......................................... 46

3.1. Estrategia: Ciclo de Mejoramiento de la Productividad................................. 46 
3.2. Herramienta: Carta Balance ….............................................................. 47

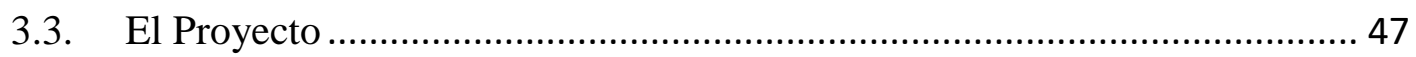

3.4. Descripción de las Partidas Analizadas en el Caso de Estudio ..................... 48

3.4.1. Enchape de Cerámicos................................................................... 49

3.4.2. Asentado de Ladrillo Blanco ............................................................. 53

3.5. Levantamiento de Información y Análisis de Resultados .......................... 57

Capítulo IV: Propuesta de Mejora y Análisis de los Resultados .................................. 64

4.1 Modelo propuesto de mejora de la situación.............................................. 64

4.2 Planes de acción para la implementación de la propuesta ........................... 65

4.3 Resultados después de la implementación de la mejora ............................ 69

4.4 Evaluación económica de la propuesta ........................................................... 74

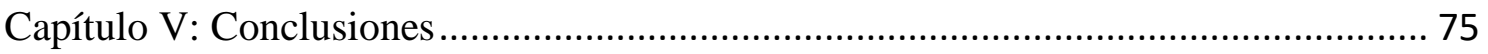

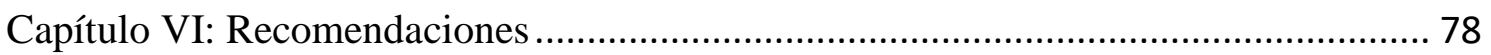

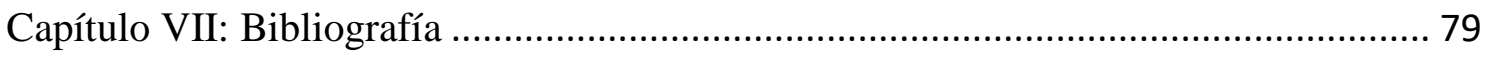

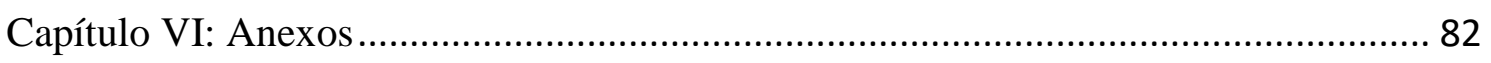




\section{ÍNDICE DE FIGURAS}

Figura $\mathrm{N}^{\mathrm{o}} 1$ : Principales relaciones de la productividad.............................................. 20

Figura $\mathrm{N}^{\mathrm{o}} 2$ : Esquema del Sistema Productivo............................................................. 21

Figura No 3: Principales Causas de Pérdidas en la Productividad.................................. 22

Figura $N^{\circ}$ 4: Principales Categorías de Pérdidas en la Productividad............................. 25

Figura $N^{o}$ 5: Composición normal del contenido de trabajo (Serpell, 2002). ................ 27

Figura $\mathrm{N}^{\mathrm{o}}$ 6: Composición ideal del contenido de trabajo (Serpell, 2002)..................... 28

Figura No 7: Promedios generales de categorías de trabajo en Chile (Serpell, 2002).... 29

Figura No 8: Distribución de categorías de trabajo en Chile (Botero \& Álvarez, 2003) 30

Figura No 9: Distribución del tiempo no contributivo en Chile (Botero \& Álvarez, 2003)

Figura No 10: Distribución de categoría de trabajo - Colombia (Botero \& Álvarez, 2003)

Figura No 11: Distribución del tiempo no contributivo - Colombia (Botero \& Álvarez,

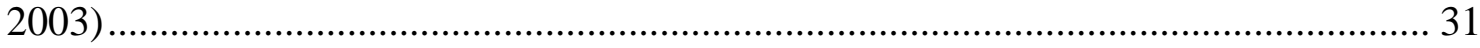

Figura No 12: Distribución de categorías de trabajo en Perú (Ghio, 2001) ..................... 33

Figura No 13: Distribución de categorías de trabajo en Perú (Morales \& Galeas, 2006)36

Figura No 14: Ciclo de mejoramiento de la productividad (Serpell, 2002) ..................... 39

Figura No 15: Procedimiento del Análisis de Métodos (Harris \& McCaffer, 2005) ...... 42

Figura No 16: Esquema del Sistema Last Planner (Rodriguez, Alarcón, \& Pellicer, 2013)

Figura No 17: Edificio Residencial Twenty - HUBY Inmobiliaria................................ 47

Figura N ${ }^{\circ}$ 18: Distribución de Trabajo en Asentado de Ladrillo.................................... 58

Figura No 19: Distribución de Trabajo Productivo en Asentado de Ladrillo. ................. 58

Figura No 20: Distribución de Trabajo Contributorio en Asentado de Ladrillo .............. 59

Figura No 21: Distribución de Trabajo No Contributorio en Asentado de Ladrillo ........ 59

Figura $N^{o}$ 22: Distribución de Trabajo en Enchape de Cerámico .................................. 61

Figura No 23: Distribución de Trabajo Productivo en Enchape de Cerámico ................. 61

Figura Nº 24: Distribución de Trabajo Contributorio en Enchape de Cerámico............. 62

Figura No 25: Distribución de Trabajo No Contributorio en Enchape de Cerámico...... 62 
Figura $N^{0}$ 26: Identificación de Problemas y Propuesta de Solución - Enchape de

Cerámico 65

Figura $N^{\circ}$ 27: Flujo de trabajo Asentado de Ladrillo Blanco..... 68

Figura No 28: Flujo de trabajo Enchape de Cerámico 68

Figura $N^{\circ}$ 29: Distribución Promedio de Trabajo de Asentado de Ladrillo - Post Implementación de Mejoras 69

Figura No 30: Distribución Promedio de Trabajo Productivo de Asentado de Ladrillo Post Implementación de Mejoras 69

Figura No 31: Distribución Promedio de Trabajo Contributorio de Asentado de Ladrillo Post Implementación de Mejoras 70

Figura $N^{\circ}$ 32: Distribución de Trabajo No Contributorio - Carta Balance ${ }^{\circ} 22$............... 70

Figura $N^{\circ}$ 33: Distribución Promedio de Trabajo de Enchape de Cerámico - Post Implementación de Mejoras 71

Figura No 34: Distribución Promedio de Trabajo Productivo de Enchape de Cerámico Post Implementación de Mejoras 71

Figura No 35: Distribución Promedio de Trabajo Contributorio de Enchape de Cerámico - Post Implementación de Mejoras 72

Figura $N^{\circ}$ 36: Distribución Promedio de Trabajo No Contributorio de Enchape de Cerámico - Post Implementación de Mejoras 72

Figura $\mathrm{N}^{\mathrm{o}}$ 37: TP, TC y TCN antes y después de la implementación de la mejora......... 75

Figura No 38: Proceso Propuesto para Incremento de la Productividad.......................... 76

Figura $N^{\circ}$ 39: Distribución de Trabajo de Asentado de Ladrillo - Carta Balance ${ }^{\circ} 1$..... 83

Figura $N^{\circ} 40$ : Distribución de Trabajo Productivo - Carta Balance ${ }^{\circ} 1$............................ 83

Figura No 41: Distribución de Trabajo Contributorio - Carta Balance ${ }^{\circ} 1$........................ 84

Figura $N^{\circ} 42$ : Distribución de Trabajo No Contributorio - Carta Balance ${ }^{\circ} 1$................. 84

Figura No 43: Distribución de Trabajo de Asentado de Ladrillo - Carta Balance ${ }^{\circ} 2$..... 86

Figura $N^{\circ}$ 44: Distribución de Trabajo Productivo - Carta Balance ${ }^{\circ} 2$........................... 86

Figura No 45: Distribución de Trabajo Contributorio - Carta Balance ${ }^{\circ} 2$....................... 87

Figura No 46: Distribución de Trabajo No Contributorio - Carta Balance ${ }^{\circ} 2$................. 87

Figura No 47: Distribución de Trabajo de Asentado de Ladrillo - Carta Balance ${ }^{\circ} 3$...... 89

Figura No 48: Distribución de Trabajo Productivo - Carta Balance ${ }^{\circ} 3$............................ 89

Figura No 49: Distribución de Trabajo Contributorio - Carta Balance ${ }^{\circ 3}$........................ 90 
Figura $N^{\circ}$ 50: Distribución de Trabajo No Contributorio - Carta Balance ${ }^{\circ 3}$.................. 90

Figura $N^{\circ}$ 51: Distribución de Trabajo de Asentado de Ladrillo - Carta Balance ${ }^{\circ} 4$..... 92

Figura No 52: Distribución de Trabajo Productivo - Carta Balance ${ }^{\circ} 4$........................... 92

Figura $N^{0}$ 53: Distribución de Trabajo Contributorio - Carta Balance ${ }^{\circ} 4$....................... 93

Figura No 54: Distribución de Trabajo No Contributorio - Carta Balance ${ }^{\circ} 4$................. 93

Figura No 55: Distribución de Trabajo de Asentado de Ladrillo - Carta Balance ${ }^{\circ} 5$..... 95

Figura No 56: Distribución de Trabajo Productivo - Carta Balance ${ }^{\circ} 5$............................ 95

Figura No 57: Distribución de Trabajo Contributorio - Carta Balance ${ }^{\circ} 5$......................... 96

Figura No 58: Distribución de Trabajo No Contributorio - Carta Balance ${ }^{\circ} 5$.................. 96

Figura $N^{\circ}$ 59: Distribución de Trabajo de Asentado de Ladrillo - Carta Balance ${ }^{\circ} 6$...... 98

Figura $N^{\circ}$ 60: Distribución de Trabajo Productivo - Carta Balance ${ }^{\circ} 6$........................... 98

Figura $N^{\circ}$ 61: Distribución de Trabajo Contributorio - Carta Balance ${ }^{\circ} 6$....................... 99

Figura $N^{\circ}$ 62: Distribución de Trabajo No Contributorio - Carta Balance ${ }^{\circ} 6$.................. 99

Figura Nº 63: Distribución de Trabajo de Asentado de Ladrillo - Carta Balance ${ }^{\circ} 7$... 101

Figura N ${ }^{\circ}$ 64: Distribución de Trabajo Productivo - Carta Balance ${ }^{\circ} 7$......................... 101

Figura No 65: Distribución de Trabajo Contributorio - Carta Balance ${ }^{\circ} 7$...................... 102

Figura Nº 66: Distribución de Trabajo No Contributorio - Carta Balance ${ }^{\circ} 7$................ 102

Figura No 67: Distribución de Trabajo de Asentado de Ladrillo - Carta Balance 8 ... 104

Figura No 68: Distribución de Trabajo Productivo - Carta Balance ${ }^{\circ} 8$.......................... 104

Figura $N^{\circ}$ 69: Distribución de Trabajo Contributorio - Carta Balance ${ }^{\circ} 8$...................... 105

Figura No 70: Distribución de Trabajo No Contributorio - Carta Balance ${ }^{\circ} 8$................ 105

Figura No 71: Distribución de Trabajo de Asentado de Ladrillo - Carta Balance ${ }^{\circ} 22$. 107

Figura No 72: Distribución de Trabajo Productivo - Carta Balance ${ }^{\circ} 22$....................... 107

Figura No 73: Distribución de Trabajo Contributorio - Carta Balance ${ }^{\circ} 22$................... 108

Figura N $N^{\circ} 74$ : Distribución de Trabajo No Contributorio - Carta Balance ${ }^{\circ} 22$............ 108

Figura N $N^{\circ}$ 75: Distribución de Trabajo de Asentado de Ladrillo - Carta Balance ${ }^{\circ} 23$. 110

Figura No 76: Distribución de Trabajo Productivo - Carta Balance ${ }^{\circ} 23$....................... 110

Figura No 77: Distribución de Trabajo Contributorio - Carta Balance ${ }^{\circ} 23$.................... 110

Figura No 78: Distribución de Trabajo No Contributorio - Carta Balance ${ }^{\circ} 23$............. 111

Figura No 79: Distribución de Trabajo de Asentado de Ladrillo - Carta Balance ${ }^{\circ} 24$. 113

Figura No 80: Distribución de Trabajo Productivo - Carta Balance ${ }^{\circ} 24$....................... 113

Figura No 81: Distribución de Trabajo Contributorio - Carta Balance ${ }^{\circ} 24$................... 114 
Figura Nº 82: Distribución de Trabajo No Contributorio - Carta Balance ${ }^{\circ} 24$

Figura N 83: Distribución de Trabajo de Enchape de Cerámico - Carta Balance `9 .. 116

Figura No 84: Distribución de Trabajo Productivo - Carta Balance ${ }^{\circ} 9$ 116

Figura N ${ }^{\circ}$ 85: Distribución de Trabajo Contributorio - Carta Balance ${ }^{\circ} 9$..................... 117

Figura No 86: Distribución de Trabajo No Contributorio - Carta Balance ${ }^{\circ} 9$............... 117

Figura No 87: Distribución de Trabajo de Enchape de Cerámico - Carta Balance 10119

Figura No 88: Distribución de Trabajo Productivo - Carta Balance ${ }^{\circ} 10$........................ 119

Figura N 89: Distribución de Trabajo Contributorio - Carta Balance ${ }^{\circ} 10$................... 120

Figura No 90: Distribución de Trabajo No Contributorio - Carta Balance ${ }^{\circ} 10$.............. 120

Figura N 91: Distribución de Trabajo de Enchape de Cerámico - Carta Balance `11 122

Figura No 92: Distribución de Trabajo Productivo - Carta Balance ${ }^{\circ} 11$....................... 122

Figura $N^{\circ}$ 93: Distribución de Trabajo Contributorio - Carta Balance ${ }^{\circ} 11$.................... 123

Figura No 94: Distribución de Trabajo No Contributorio - Carta Balance ${ }^{\circ} 11$............. 123

Figura Nº 95: Distribución de Trabajo de Enchape de Cerámico - Carta Balance 12125

Figura No 96: Distribución de Trabajo Productivo - Carta Balance ${ }^{\circ} 12$........................ 125

Figura No 97: Distribución de Trabajo Contributorio - Carta Balance ${ }^{\circ} 12$.................... 126

Figura No 98:Distribución de Trabajo No Contributorio - Carta Balance ${ }^{\circledR} 12$.............. 126

Figura No 99: Distribución de Trabajo de Enchape de Cerámico - Carta Balance 13128

Figura No 100: Distribución de Trabajo Productivo - Carta Balance ${ }^{\circ} 13$..................... 128

Figura No 101: Distribución de Trabajo Contributorio - Carta Balance ${ }^{\circ} 13$................. 129

Figura N ${ }^{\circ}$ 102: Distribución de Trabajo No Contributorio - Carta Balance ${ }^{\circ} 13$........... 129

Figura Nº 103: Distribución de Trabajo de Enchape de Cerámico - Carta Balance ` 14

Figura No 104: Distribución de Trabajo Productivo - Carta Balance ${ }^{\circ} 14$..................... 131

Figura No 105: Distribución de Trabajo Contributorio - Carta Balance ${ }^{\circ} 14$................. 132

Figura No 106: Distribución de Trabajo No Contributorio - Carta Balance ${ }^{\circ} 14$........... 132

Figura No 107: Distribución de Trabajo de Enchape de Cerámico - Carta Balance 15

Figura No 108: Distribución de Trabajo Productivo - Carta Balance ${ }^{\circ} 15$...................... 134

Figura N N 109: Distribución de Trabajo Contributorio - Carta Balance ${ }^{\circ} 15$................. 135

Figura N ${ }^{\circ}$ 110: Distribución de Trabajo No Contributorio - Carta Balance ${ }^{\circ} 15$........... 135 
Figura Nº 111: Distribución de Trabajo de Enchape de Cerámico - Carta Balance ` 16 137

Figura N ${ }^{\circ}$ 112: Distribución de Trabajo Productivo - Carta Balance ${ }^{\circ} 16$...................... 137

Figura $N^{0}$ 113: Distribución de Trabajo Contributorio - Carta Balance ${ }^{\circ} 16$................. 138

Figura No 114: Distribución de Trabajo No Contributorio - Carta Balance ${ }^{\circ} 16$........... 138

Figura No 115: Distribución de Trabajo de Enchape de Cerámico - Carta Balance ` 17 140

Figura N 116: Distribución de Trabajo Productivo - Carta Balance ${ }^{\circ} 17$..................... 140

Figura No 117: Distribución de Trabajo Contributorio - Carta Balance ${ }^{\circ} 17$.................. 141

Figura N ${ }^{\circ}$ 118: Distribución de Trabajo No Contributorio - Carta Balance ${ }^{\circ} 17$........... 141

Figura Nº 119: Distribución de Trabajo de Enchape de Cerámico - Carta Balance ` 18

Figura No 120: Distribución de Trabajo Productivo - Carta Balance ${ }^{\circ} 18$..................... 143

Figura No 121: Distribución de Trabajo Contributorio - Carta Balance ${ }^{\circ} 18$.................. 144

Figura N 122: Distribución de Trabajo No Contributorio - Carta Balance ${ }^{\circ} 18$........... 144

Figura Nº 123: Distribución de Trabajo de Enchape de Cerámico - Carta Balance ` 19

Figura No 124: Distribución de Trabajo Productivo - Carta Balance ${ }^{\circ} 19$..................... 146

Figura No 125: Distribución de Trabajo Contributorio - Carta Balance ${ }^{\circ} 19$.................. 147

Figura No 126: Distribución de Trabajo No Contributorio - Carta Balance ${ }^{\circ} 19$........... 147

Figura N 127: Distribución de Trabajo de Enchape de Cerámico - Carta Balance ${ }^{\circ} 20$

Figura No 128: Distribución de Trabajo Productivo - Carta Balance ${ }^{\circ} 20$..................... 149

Figura N ${ }^{\circ}$ 129: Distribución de Trabajo Contributorio - Carta Balance ${ }^{\circ} 20$................. 150

Figura No 130: Distribución de Trabajo No Contributorio - Carta Balance ${ }^{\circ} 20$........... 150

Figura Nº 131: Distribución de Trabajo de Enchape de Cerámico - Carta Balance ${ }^{\circ} 21$

Figura No 132: Distribución de Trabajo Productivo - Carta Balance ${ }^{\circ} 21$...................... 152

Figura No 133: Distribución de Trabajo Contributorio - Carta Balance ${ }^{\circ} 21$................. 153

Figura No 134: Distribución de Trabajo No Contributorio - Carta Balance ${ }^{\circ} 21$........... 153 


\section{ÍNDICE DE TABLAS}

Tabla No 1: Principales Categorías de Pérdidas de Productividad 25

Tabla No 2: Resultado de la distribución de categorías de trabajo en las 50 obras en Lima (Ghio, 2001) 32

Tabla No 3: Principales causas de pérdidas de la construcción en el Perú (Ghio, 2001) 34 Tabla No 4: Porcentaje de tiempo empleado por actividad. (Morales \& Galeas, 2006). 36 Tabla No 5: Comparativo de Sistema Last Planner y Sistema Tradicional. (Kalsaas, Grindheim y Laeknes, 2014) 45

Tabla No 6: Clasificación de las actividades de Enchape de Cerámico........................... 52

Tabla N $N^{\circ}$ : Clasificación de las actividades de Asentado de Ladrillo Blanco................ 56

Tabla No 8: Clasificación de las actividades de Asentado de Ladrillo Blanco................ 57

Tabla No 9: Identificación de Problemas y Propuesta de Solución - Asentado de Ladrillo Blanco 64

Tabla No 10: Comparación de los TP, TC y TNC de las partidas analizadas 73

Tabla N ${ }^{0}$ 11: Estimado de costos antes y después de la implementación de la mejora Asentado de Ladrillo Blanco

Tabla $\mathrm{N}^{\mathrm{0}}$ 12: Estimado de costos antes y después de la implementación de la mejora Enchape de Cerámico 74

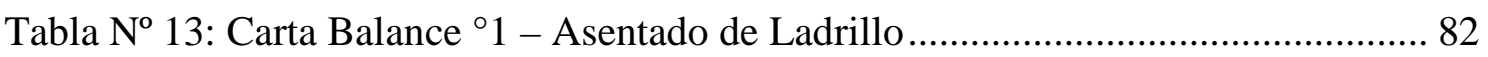

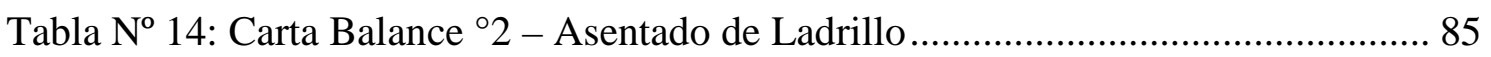

Tabla N 15: Carta Balance ${ }^{\circ} 3$ - Asentado de Ladrillo ................................................... 88

Tabla No 16: Carta Balance ${ }^{\circ} 4$ - Asentado de Ladrillo ..................................................... 91

Tabla No 17: Carta Balance ${ }^{\circ} 5$ - Asentado de Ladrillo ..................................................... 94

Tabla No 18: Carta Balance ${ }^{\circ} 6$ - Asentado de Ladrillo ..................................................... 97

Tabla N ${ }^{\circ}$ 19: Carta Balance ${ }^{\circ} 7$ - Asentado de Ladrillo................................................. 100

Tabla No 20: Carta Balance ${ }^{\circ} 8$ - Asentado de Ladrillo ................................................... 103

Tabla No 21: Carta Balance ${ }^{\circ} 22$ - Asentado de Ladrillo.............................................. 106

Tabla No 22: Carta Balance ${ }^{\circ} 23$ - Asentado de Ladrillo............................................. 109

Tabla N 23: Carta Balance ${ }^{\circ} 24$ - Asentado de Ladrillo............................................... 112

Tabla No 24: Carta Balance ${ }^{\circ} 9$ - Enchape de Cerámico................................................ 115

Tabla No 25: Carta Balance ${ }^{\circ} 10$ - Enchape de Cerámico................................................ 118 
Tabla Nº 26: Carta Balance ${ }^{\circ} 11$ - Enchape de Cerámico............................................. 121

Tabla No 27: Carta Balance ${ }^{\circ} 12$ - Enchape de Cerámico............................................... 124

Tabla No 28: Carta Balance ${ }^{\circ} 13$ - Enchape de Cerámico............................................... 127

Tabla No 29: Carta Balance ${ }^{\circ} 14$ - Enchape de Cerámico............................................. 130

Tabla No 30: Carta Balance ${ }^{\circ} 15$ - Enchape de Cerámico............................................. 133

Tabla No 31: Carta Balance ${ }^{\circ} 16$ - Enchape de Cerámico............................................. 136

Tabla No 32: Carta Balance ${ }^{\circ} 17$ - Enchape de Cerámico.............................................. 139

Tabla No 33: Carta Balance ${ }^{\circ} 18$ - Enchape de Cerámico................................................ 142

Tabla No 34: Carta Balance ${ }^{\circ} 19$ - Enchape de Cerámico................................................ 145

Tabla No 35: Carta Balance ${ }^{\circ} 20$ - Enchape de Cerámico................................................ 148

Tabla No 36: Carta Balance ${ }^{\circ} 21$ - Enchape de Cerámico............................................. 151 


\section{ÍNDICE DE FOTOGRAFÍAS}

Fotografía $\mathrm{N}^{\mathrm{o}}$ 1: Enchape de Cerámicos - Edificio Twenty......................................... 50

Fotografía N $\mathrm{N}^{\mathrm{0}}$ 2Enchape de Cerámicos - Edificio Twenty.......................................... 50

Fotografía No 3: Enchape de Cerámicos - Edificio Twenty....................................... 51

Fotografía No 4: Enchape de Cerámicos - Edificio Twenty....................................... 51

Fotografía No 5: Asentado de Ladrillo Blanco - Edificio Twenty................................. 54

Fotografía No 6: Asentado de Ladrillo Blanco - Edificio Twenty.................................. 54

Fotografía $\mathrm{N}^{\circ}$ 7: Asentado de Ladrillo Blanco - Edificio Twenty................................. 55

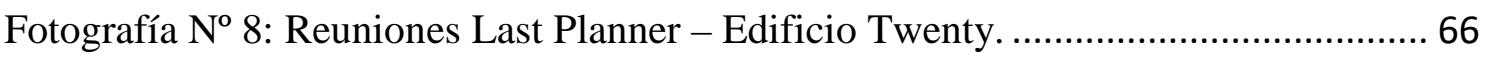

Fotografía No 9: Liberación de restricciones con los jefes de grupo - Edificio Twenty. 66

Fotografía Nº 10: Planificación semanal y liberación de restricciones - Edificio Twenty. 67

Fotografía No 11: Resultado de planificación semanal - Edificio Twenty. 67 


\section{INTRODUCCIÓN}

En el presente trabajo de investigación se aborda la implementación de mejoramiento para reducir el trabajo no contributorio en el caso de estudio: Vivienda Multifamiliar “Twenty en el distrito de Lince - Lima”. Se estima que aproximadamente el 30\% del costo de construcción de un edificio es la mano de obra (Heinrich, 2018), esto hace que sea importante el análisis de esta parte de la construcción.

La investigación de este tema se ha realizado por el interés de poder mejorar los tiempos de producción y reducir los tiempos no contributorios en la construcción, es que en la actualidad los proyectos inmobiliarios se han visto obligados a mejorar sus procesos de construcción, ya que en los últimos años hubo una caída en el sector construcción. Esto ha obligado a que las empresas constructoras tengan que reducir los gastos de producción, buscando y analizando herramientas que optimicen su producción, para ser más competitivos en el mercado y ofrecer un menor precio de venta.

Para lograr el objetivo, en el presente trabajo de investigación, se usó la herramienta Carta Balance, con el fin de medir los trabajos productivos, contributorios y no contributorios, posteriormente se realizó el análisis haciendo uso de herramientas de Lean Contruction y análisis de flujos de trabajo obteniendo como resultados las propuestas de mejoramiento (planes de trabajo).

Los resultados obtenidos luego de la implementación de las mejoras mostraron una reducción del trabajo no contributorio de aproximadamente un 5\% y 31\% en las partidas de Asentado de Ladrillo y Enchape de Cerámico, respectivamente. Además, se observa un ahorro económico del orden 30\% aproximadamente en las partidas mencionadas líneas arriba. 


\section{CAPÍTUlo I: ASPECTOS GENERALES}

\subsection{El Problema.}

En la actualidad, la construcción es uno de los principales sectores que impulsan el crecimiento de la economía en el Perú y se postula como el sector económico que liderará el crecimiento del PBI en los siguientes años debido a su continuo desarrollo (IEDEP, 2018). Sin embargo, el sector de la construcción en el Perú presenta deficiencias en la construcción, lo que genera una falta de efectividad al momento de la ejecución de los proyectos.

Uno de los objetivos de las empresas de construcción es mejorar los índices de productividad para garantizar el éxito en la ejecución de sus proyectos. Un factor fundamental para lograr esta meta consiste en controlar y disminuir de manera efectiva los trabajos no contributorios, que son las actividades que no tienen ningún aporte a la ejecución de alguna unidad de construcción y que no son necesarias. Sin embargo, en la actualidad, el porcentaje de trabajos no contributorios involucra, aproximadamente, un tercio del total de trabajos que se ejecutan en las obras. Por lo tanto, la productividad en el sector de la construcción no evoluciona a pesar del desarrollo económico constante que tiene (Santana, 1989).

Según Santana (1989), uno de los principales factores que incurren en los trabajos no contributorios en obra es el empleo del sistema tradicional para la gestión de la producción en obra, el cual no incluye un control continuo de las actividades, así como no existe un análisis y eliminación de restricciones. Esto se traduce en una planificación y programación deficiente de actividades (esto genera esperas por materiales y/o indicaciones, traslados innecesarios, retrabajos, reprocesos, entre otros). Del mismo modo, Serpell (2002) señala que existen factores críticos que inciden en los trabajos no contributorios, como la espera por las herramientas y/o materiales necesarios para la ejecución de su trabajo y el empleo de métodos de trabajo ineficientes. Por otro lado, según estudios realizados en Colombia, se muestra que el trabajo no contributorio se origina principalmente porque no existe una planificación eficiente para la entrega y localización de los recursos para las cuadrillas de trabajo, lo que se traduce en tiempo 
perdido en esperas y transportes (Botero \& Álvarez, Identificación de Pérdidas en el Proceso Productivo de la Construcción, 2003).

El efecto que tiene el aumento de los trabajos no contributorios incide principalmente en el presupuesto del proyecto. Como señala Enshassi (2013), la baja productividad es un problema alarmante debido a su impacto sobre los costos, así como en la competitividad de la empresa en el mercado y la viabilidad de la planificación y programación del proyecto. Asimismo, Enshassi (2007) indica que los trabajos no contributorios es una de las principales causas por la que las empresas de construcción no logran sus objetivos en relación a las utilidades. Por otro lado, Ponz-Tienda (2014) presenta un estudio en España que muestra que el costo de la mano de obra representa un $30 \%$ a $50 \%$ del presupuesto total del proyecto, así que la efectividad con la que empleas este recurso es fundamental para reducir este porcentaje dentro del presupuesto.

Debido al impacto que tiene los trabajos no contributorios en el sector de la construcción, se han realizado diversas investigaciones en relación a este problema en el Perú. El Ing. Virgilio Ghio (2000), realizó una investigación sobre la proporción de trabajos productivos, contributorios y no contributorios que existe en las obras de construcción en el Perú (en función a 50 obras analizadas). Como resultado, el 36\% del tiempo en obra se realizan trabajos no contributorios, es decir, una tercera parte de la jornada laboral no tiene producción alguna. Asimismo, Morales \& Galeas (2006) investigaron sobre el problema en función a 26 obras analizadas. Los resultados mostraron que existe un 25\% del tiempo que se ejecutan trabajos no contributorios. Cabe señalar, que existen investigaciones que indican que el valor óptimo del tiempo empleado por trabajos no contributorios durante la jornada debe ser de un 15\%. Por esta razón, según los muestreos observados, el índice de trabajo no contributorio es muy alto, 25\% - 35\%, en relación al valor objetivo de 15\% (Ghio, 2001).

\subsection{Formulación del Problema.}

El alto porcentaje de trabajo no contributorio en las obras de construcción de edificaciones debido a las ineficiencias administrativas que existen en las obras (planificación y programación deficiente de actividades y/o materiales). 


\subsection{ObJetivo General.}

Implementar una propuesta de mejoramiento, en el proyecto de Vivienda Multifamiliar TWENTY en el distrito de Lince - Lima, para reducir el porcentaje del trabajo no contributorio.

\subsection{OBJetivo ESPECíficos.}

- Medir y analizar los resultados obtenidos en relación a los trabajos no contributorios en el caso de estudio, antes y después de implementar las propuestas de mejora.

- Plantear la implementación de un proceso de mejoramiento, con las herramientas de Last Planner, para reducir los trabajos no contributorios.

- Identificar los costos y beneficios de la presente implementación.

\subsection{Justificación del Problema.}

Según lo mencionado anteriormente, diversas investigaciones evidencian que existen obras de construcción con un porcentaje elevado (25\% - 35\%) de trabajos no contributorios con respecto al total de trabajos. Esto se debe a distintos factores como la ineficiencia administrativa, la motivación del personal obrero, necesidades fisiológicas, entre otros. Como consecuencia, el alto porcentaje de trabajos no contributorios tiene un impacto negativo en el presupuesto y plazo en el proyecto, ya que la mano de obra es el recurso con mayor incidencia en estos dos aspectos.

Debido a la problemática en mención, se realizará una propuesta de proceso de mejoramiento para reducir el porcentaje de los trabajos no contributorios y, de esta forma, ser más eficientes en la ejecución. Asimismo, este proceso se implementará en el caso de estudio "Vivienda Multifamiliar TWENTY" en el distrito de Lince - Lima.

\subsection{AlCANCES y Limitaciones.}

El trabajo de investigación se limitará a la reducción del trabajo no contributorio, mediante el uso de herrmaientas de Lean Contruction y análisis de flujos, en la obra: "Vivienda Multifamiliar TWENTY” en el distrito de Lince - Lima. 


\subsection{MEtodología de INVESTIGACión.}

Para el presente trabajo de investigación, se realizará el análisis del estado actual de los trabajos: productivo, contributorio y no contributorio, del proyecto elegido como caso de estudio. Para ello se hará uso de herramientas como la carta balance. De esta forma, se tendrá el punto de partida el cual será comparado con el resultado final, luego de implementar las mejoras propuestas.

Luego de la implementación de las soluciones propuestas se realizará la medición final para ver el efecto en los indicadores de: trabajo productivo, trabajo contributorio y no contributorio. 


\section{CAPÍTULO II: MARCO TEÓRICO}

\subsection{Productividad en la Construcción.}

Hoy en día, en el sector de la construcción se menciona con más frecuencia los términos relacionados con la productividad, puesto que, actualmente, existen empresas constructoras que buscan ser más productivas para durar a lo largo del tiempo en un mercado altamente competitivo. Por este motivo, las empresas constructoras están en la constante búsqueda de métodos y/o herramientas que permitan ejecutar las obras con mayor efectividad, disminuyendo los sobrecostos y los reprocesos.

Asimismo, gran parte de las metodologías modernas de mejoramiento en el sector de la construcción tienen como base de su estrategia maximizar el valor y minimizar las pérdidas, por lo que la productividad es uno de los indicadores más influyentes para medir el desempeño de la gestión de la obra. Incluir a la productividad dentro de la estrategia de la gestión en las obras es fundamental para medir el desarrollo constructivo, ya que involucra diversos elementos claves que tienen relación directa con el desempeño de los procesos, como la planificación, la calidad, el costo, el tiempo, el control y la seguridad. Asimismo, la productividad en construcción involucra la productividad en sus procesos, recursos, mano de obra o cuadrillas de trabajo, equipos, entre otros (Mejía \& Hernández, 2007). A continuación, en la Figura $N^{\circ} 1$ se presenta las principales relaciones de la productividad de la construcción.

Figura $N^{o} 1$ : Principales relaciones de la productividad.

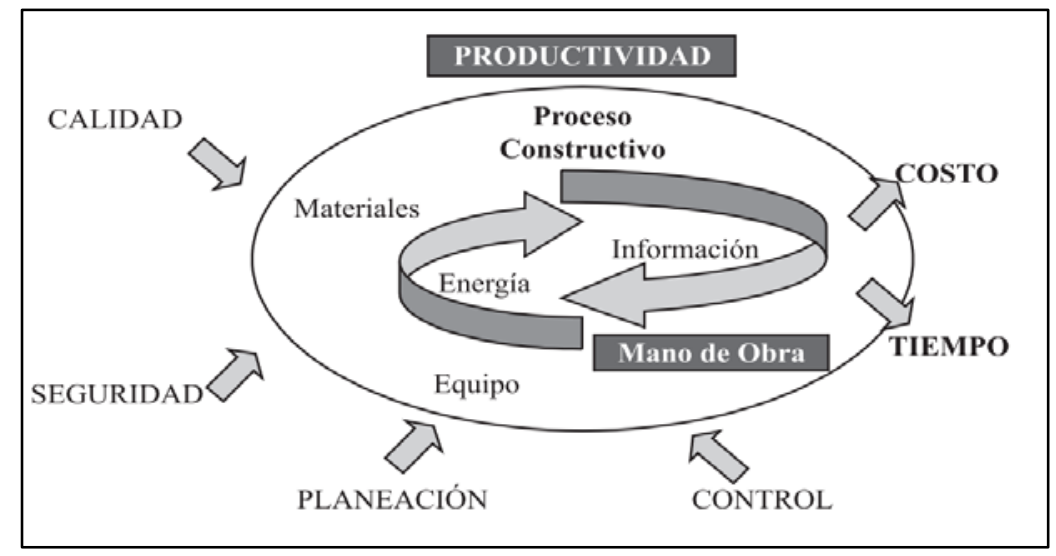


Por lo tanto, según lo descrito anteriormente, a la productividad se le entiende como un indicador de efectividad en un sistema o proceso, en el cual, relaciona la eficacia y la eficiencia dentro de un marco sinérgico. En este caso, la eficacia se presenta como el logro de obtener un producto con un alcance definido, el cual cumple con las condiciones estándares de calidad y con un plazo de entrega determinado. Asimismo, la efectividad se representa como el aprovechamiento de los recursos empleado, con el fin de obtener el producto mencionado con el menor costo posible. En síntesis, la productividad es un indicador que mide la eficiencia con la que se emplea los recursos disponibles para ejecutar y culminar un producto específico, dentro de los estándares de calidad y en un plazo determinado (Mejía \& Hernández, 2007).

El indicador de productividad consiste en la relación entre la cantidad de trabajo producido con respecto a los recursos utilizados:

$$
\text { PRODUCTIVIDAD }=\frac{\text { CANTIDAD EJECUTADA }}{\text { RECURSOS EMPLEADOS }}
$$

Una productividad mayor se traduce en ejecutar más trabajo con la misma cantidad de recursos o también consiste en realizar lo mismo, pero con menos recursos.

Como se mencionó anteriormente, el objetivo de las empresas constructoras es lograr alcanzar una alta productividad en los procesos productivos (o por lo menos en los más incidentes). Estos procesos cuentan con recursos que son transformados en productos esperados (Serpell, 2002). En la Figura $N^{\circ} 2$ se muestra el esquema del sistema productivo en general.

Figura $N^{\circ} 2$ : Esquema del Sistema Productivo.

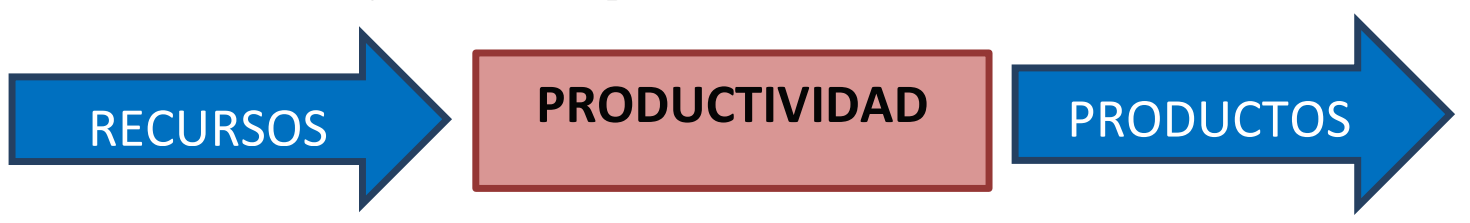

En el caso de la construcción, los principales recursos empleados dentro de este sistema productivo son los siguientes:

- Mano de Obra

- Materiales y Herramientas 
- Equipos y Maquinarias

- Información

En base a los recursos mencionados, es necesario considerar la productividad en cada uno de ellos dentro de la ejecución de la obra de construcción. En el caso de los materiales es fundamental evitar los desperdicios, como es en el caso del concreto, arena fina, arena gruesa, ladrillos, cemento, entre tantos otros insumos que se emplean en las construcciones. Asimismo, en el caso de los equipos y mano de obra es importante controlar los tiempos muertos o no productivos que estos tengan (Botero, 2004).

Por otro lado, existen diferentes factores que afectan la productividad los cuales se denominan “pérdidas”. Se consideran pérdidas o desperdicios a las actividades humanas que uizan recursos de manera ineficiente y, por lo tanto, no crean valor al producto. Según Serpell (2002), estas “pérdidas” en la productividad se clasifican en siete categorías, las cuales se presentan en forma de esquema en la Figura $\mathrm{N}^{\circ} 3$.

Figura No 3: Principales Causas de Pérdidas en la Productividad.

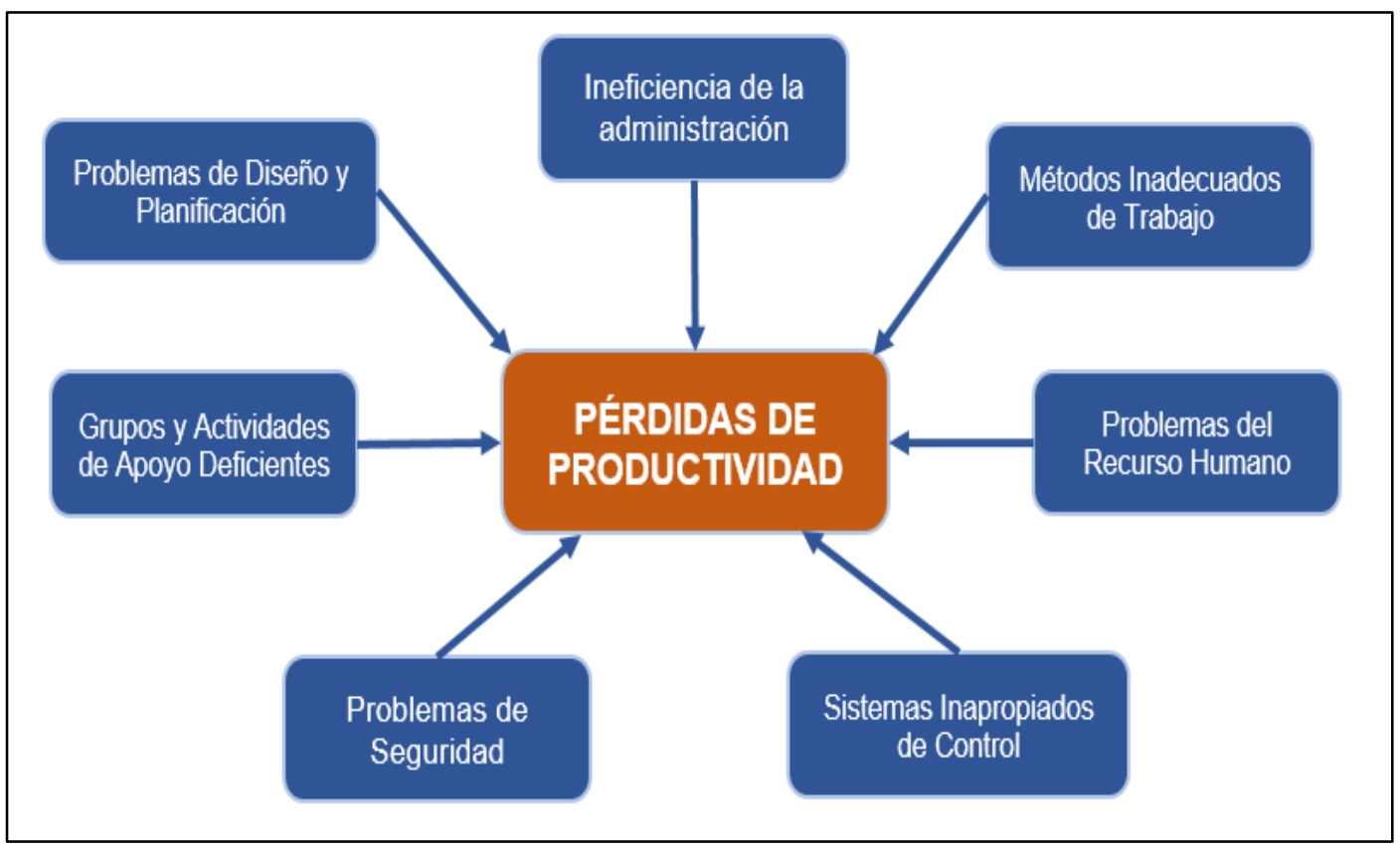

A continuación, se describirá ejemplos de cada una de las principales causas de pérdidas en la productividad de las obras de construcción según Serpell (2002):

a) Problemas de diseño y planificación: 
- Deficiencias en la interfase Ingeniería-Construcción, que consisten en los problemas de diseño.

- Falta de planificación preliminar y operacional para la ejecución de la obra.

- Falta de información y herramientas para la adecuada gestión de la obra.

- Poca constructibilidad de los diseños, así como de los métodos de construcción.

b) Ineficiencia de la administración:

- Falta de supervisión efectiva de los trabajos.

- Problemas de coordinación y comunicación entre las distintas áreas de trabajo.

- Incorrecta planificación de los trabajos a ejecutar.

- La administración tiende a ser más reactiva (“apagar incendios”) que preventiva.

c) Métodos inadecuados de trabajo:

- Ineficiente empleo de los recursos asignados.

- Deficiente uso de las tecnologías para el trabajo.

- Falta de innovación para mejorar procesos de trabajo.

- Baja calidad de los trabajos ejecutados.

- Falta de lecciones aprendidas y no existe una mejora continua en los proyectos.

d) Grupos y actividades de apoyo deficientes:

- Falta de recursos para ejecutar los trabajos.

- No existe disponibilidad de recursos por razones del mismo mercado o por el inadecuado proceso de adquisiciones.

- Inadecuada gestión del empleo de los recursos, así como del control de inventarios y de las órdenes de compra.

- Falta de mantenimiento de los equipos y maquinarias.

e) Problemas del recurso humano:

- Falta de capacitación al personal, que trae consigo problemas durante la ejecución de los trabajos. 
- Inexistente o casi nula gestión del recurso humano en las obras, que se traduce en la falta de motivación del personal.

- Falta de reconocimiento a los trabajadores por la correcta ejecución de los trabajos y cumpliendo el índice de productividad planificado.

- No se tiene en cuenta la experiencia del personal.

f) Problemas de seguridad:

- Desconocimiento por parte de la administración sobre la importancia de la seguridad en las obras de construcción.

- Falta de conciencia acerca del impacto que genera un ambiente en condiciones inseguras en la motivación y productividad del personal.

g) Sistemas inapropiados de control:

- Generalmente no mide la productividad, por lo tanto no se pueden brindar acciones correctivas o mejoras en los distintos procesos de construcción.

- Falta de identificación de los problemas de productividad, por lo tanto, los problemas que existan no se pueden corregir.

- Distorsión de la información del estado actual de la obra para esconder los problemas existentes.

- Empleo de recursos que exceden el presupuesto proyectado sin tener en cuenta otros elementos que pueden ser grandes potenciales de ahorro dentro de la obra.

Estas siete principales causas de pérdida de productividad se pueden resumir en las cinco categorías de factores que reducen la productividad establecidos por el mismo Serpell (2002). Es necesario que el personal de obra conozca tengan bien en claro los factores que afectan la productividad, para que puedan disminuir o eliminar los efectos negativos que estos generan. A continuación, se presenta las cinco categorías de pérdidas de productividad en la Figura $\mathrm{N}^{\circ} 4$. 
Figura No 4: Principales Categorías de Pérdidas en la Productividad.

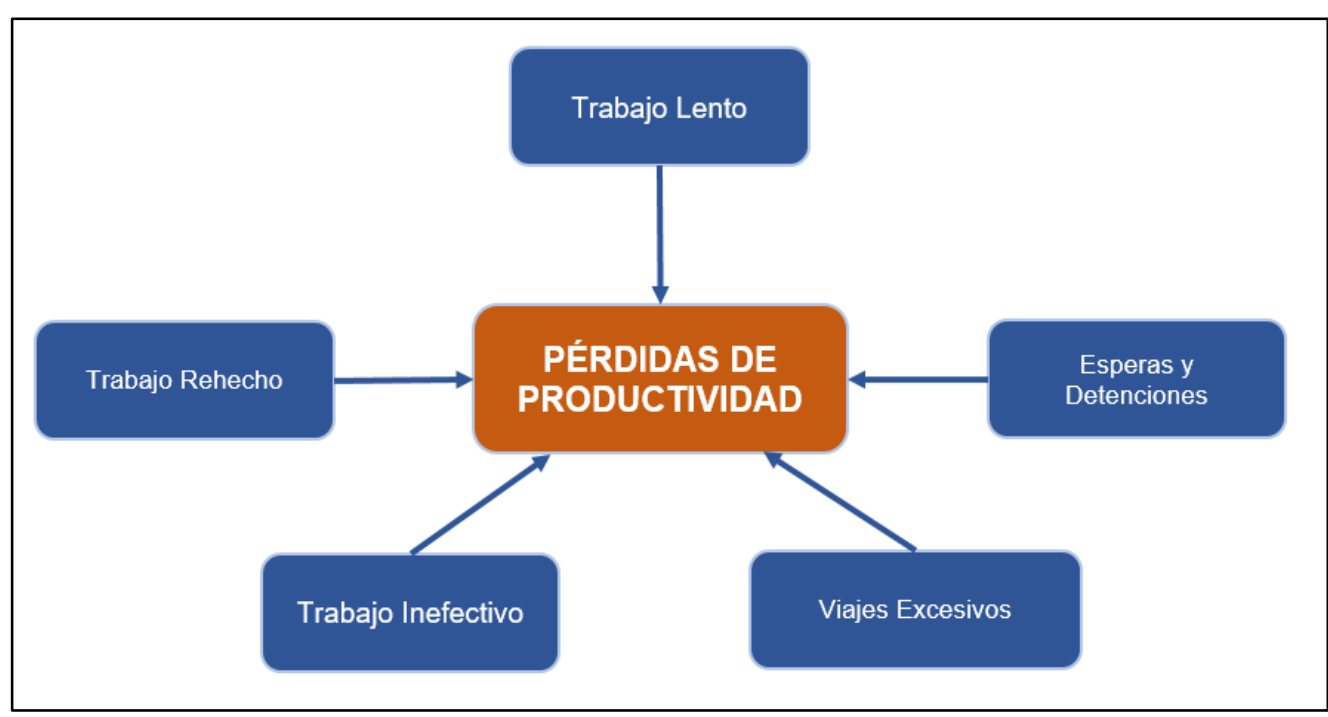

Cada una de las presentes categorías está compuesta por diversos factores que están relacionados entre sí. En la siguiente Tabla $\mathrm{N}^{\circ} 1$, se muestra algunos factores, como ejemplo, que están involucrados en cada uno de las categorías en mención (Serpell, 2002).

Tabla No 1: Principales Categorías de Pérdidas de Productividad

\begin{tabular}{|c|c|}
\hline Categoria & Factores \\
\hline \multirow{4}{*}{$\begin{array}{c}\text { Esperas y } \\
\text { Detenciones }\end{array}$} & Espera por materiales \\
\hline & Espera por información \\
\hline & Espera por indicaciones \\
\hline & Espera de uso del espacio de trabajo \\
\hline \multirow{4}{*}{ Viajes Excesivos } & Deficiente distribución de espacios \\
\hline & Caminos largos y complicados \\
\hline & Varios lugares para ejecutar un proceso \\
\hline & Ubicación inapropiada de los materiales y herramientas \\
\hline \multirow{4}{*}{ Trabajo Lento } & Falta de motivación en los obreros \\
\hline & Falta de capacitación a los obreros \\
\hline & Clima adverso y fatiga \\
\hline & Agrupamiento de trabajadores en espacios reducidos \\
\hline \multirow{2}{*}{ Trabajo Inefectivo } & Trabajos inventados para mantener ocupado al personal \\
\hline & Métodos complicados cuando existen métodos más ágiles \\
\hline \multirow{4}{*}{ Trabajo Rehecho } & Retrabajos en actividades mal ejecutadas \\
\hline & Cambios de diseño, diseños incompletos o atrasados \\
\hline & Errores y omisiones en planos y especificaciones \\
\hline & Muchas modificaciones durante la ejecución de la obra \\
\hline
\end{tabular}

Las distintas pérdidas mencionadas en el presente capítulo son o incluyen factores que de una u otra forma afecta directamente a la mano de obra, puesto que son los encargados de ejecutar las actividades planificadas. Por lo tanto, es importante conocer estos factores 
negativos dentro de la obra para tomar en cuenta las siguientes acciones y comenzar el camino para aumentar la productividad en las obras (Gómez \& Morales, 2016):

- Desarrollar el conocimiento de la mano de obra y reconocer su desempeño en el trabajo para que sean más eficientes y efectivos.

- Indicar el desempeño de la productividad en la situación actual de la obra.

- Establecer estrategias de mejora, involucrando a la gerencia de la obra, para incrementar la productividad, teniendo como base la mano de obra.

- Mejorar el ambiente y condiciones de trabajo para la mano de obra.

Cabe recalcar que dentro de todos los recursos mencionados que involucran las obras de construcción, se considera que el pilar principal para el mejoramiento de la productividad son los recursos humanos, puesto que el capital invertido o la tecnología que se emplea se desempeñarán en función a como las personas lo gestionen. A pesar de esto, no siempre se le considera con la importancia que le corresponde, considerando que la mano de obra es un recurso importante en el proceso constructivo. Asimismo, la mano de obra tiene una incidencia fuerte dentro del presupuesto de la obra. Como señaló Ponz-Tienda (2014), el costo de la mano de obra generalmente representa entre el $30 \%$ - 50\% del presupuesto total del proyecto, por lo que es importante que la gestión de este recurso sea efectiva.

\subsection{Categorías de Trabajo en la Construcción.}

Como se mencionó anteriormente, en la actualidad el sector de la construcción está buscando mejorar los índices de productividad durante la ejecución de las obras de construcción. Para aumentar estos índices, es necesario mejorar los procesos constructivos de manera que sean más efectivas y eficientes. Para esto, es necesario tener en cuenta que la ejecución de estas actividades está compuesta por trabajos que son productivos e improductivos. El contenido de trabajo de una actividad o proceso constructivos está compuesto por las siguientes categorías de trabajo (Ghio, 2001):

- Trabajo Productivo: Trabajo que aporta directamente a la ejecución de una unidad de construcción. Ejemplos: Asentar ladrillo, tarrajeo de muros, vaciar concreto, montaje de acero, encofrado de columnas, entre otros. 
- Trabajo Contributorio: Trabajo de apoyo necesario para la ejecución de los trabajos productivos. Ejemplos: Ordenar zona de trabajo, acarreo de materiales y herramientas, recibir o dar instrucciones, leer planos, armado de andamios o plataformas, remover mortero sobrante, entre otros.

- Trabajo No Contributorio: Cualquier trabajo que no genere valor y que esté dentro de las categorías de pérdidas. Ejemplos: Esperas del personal, caminar con las manos vacías, tiempo de ocio, traslado de botellas de agua y/o alimentos, necesidades fisiológicas, entre otros.

Por otro lado, en la Figura $\mathrm{N}^{\circ} 5$ se observa la composición normal del contenido de trabajo, en donde la productividad del trabajo se mide en base a la cantidad de trabajo productivo.

Figura No 5: Composición normal del contenido de trabajo (Serpell, 2002).

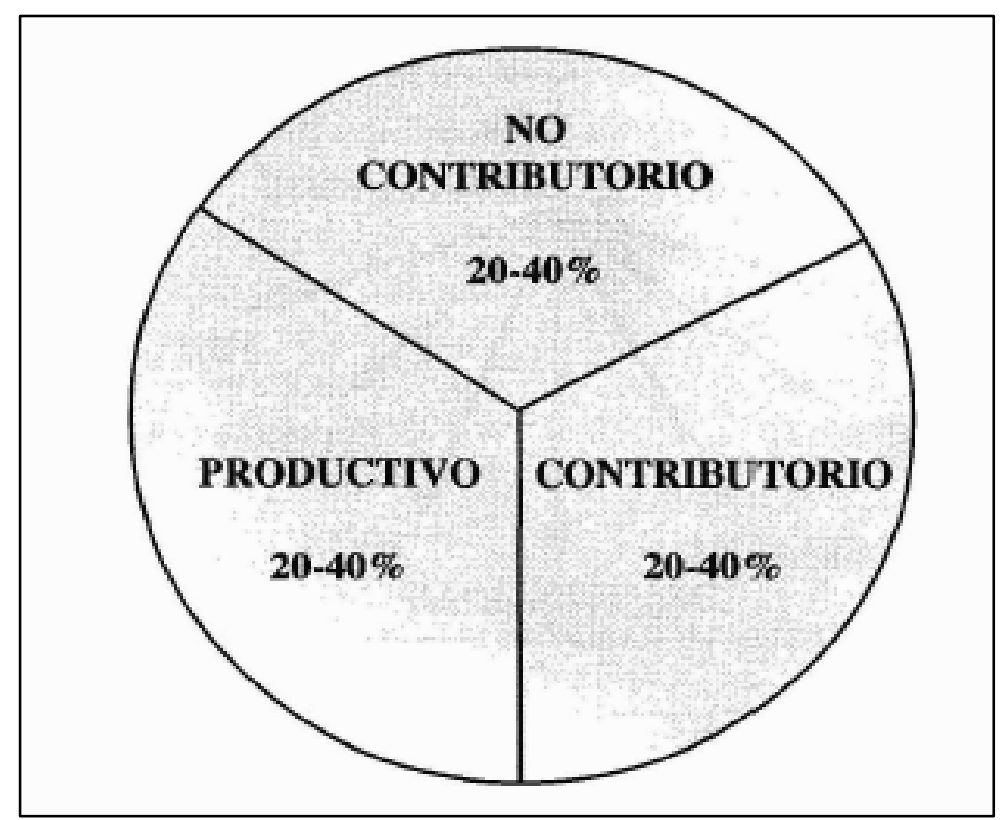

Teniendo en cuenta la composición del contenido de trabajo, Serpell (2002) realizó una investigación en varias obras que consistía en el seguimiento de los índices de trabajo de las categorías mencionadas. En las obras donde se aplicaron sistemas de mejoramiento de la productividad se alcanzaron ciertos valores que se consideran como los valores óptimos para la distribución ideal de los trabajos productivos, contributorios y no contributorios. Esta composición ideal del contenido de trabajo se muestra en la Figura $\mathrm{N}^{\circ} 6$. 
Figura Nº 6: Composición ideal del contenido de trabajo (Serpell, 2002).

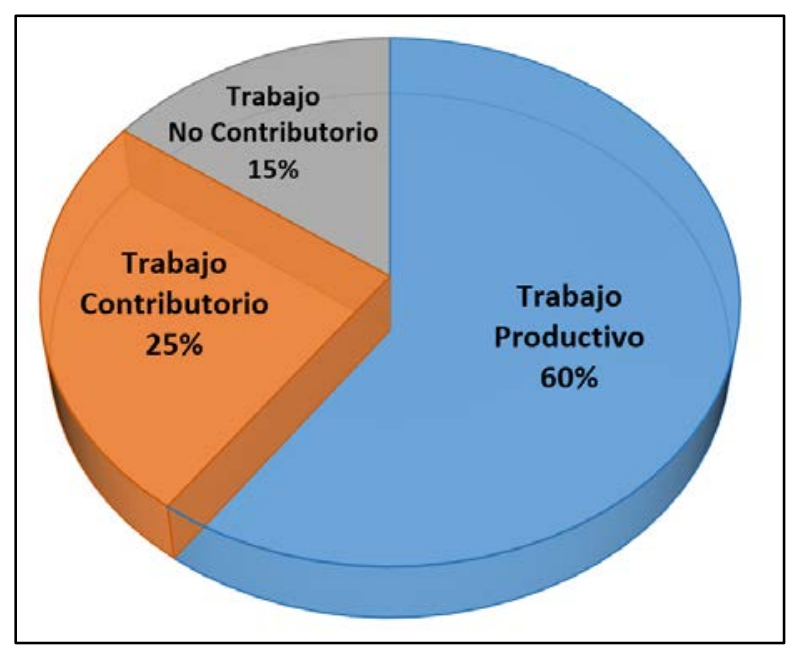

\subsubsection{Trabajo No CONTRIBUTORIO: InVESTIGACIONES Y CAUSAS}

El trabajo no contributorio se define como el porcentaje de la jornada de trabajo que involucra los tiempos "muertos" o improductivos concebidos en una obra de construcción. Los tiempos improductivos que generan los trabajos no contributorios originan ineficiencias en la gestión de los recursos empleados y en la dirección general de las obras.

Serpell (2002) presentó un análisis realizado por el Servicio de Productividad y Gestión del Departamento de Ingeniería y Gestión de la Construcción de la Pontificia Universidad Católica de Chile sobre el porcentaje de los trabajos productivos, contributorios y no contributorios que se producen durante la ejecución de las actividades. Como muestra, se tomaron 30 obras de construcción de diferentes tipos en Chile a lo largo de dos años. Cabe señalar que el número de obras era muy limitado y estas estaban atravesando por una etapa de mejoramiento de la productividad. En la siguiente Figura $\mathrm{N}^{\circ} 7$ se observa los promedios generales para cada categoría de trabajo en relación a la muestra investigada. 
Figura Nº 7: Promedios generales de categorías de trabajo en Chile (Serpell, 2002).

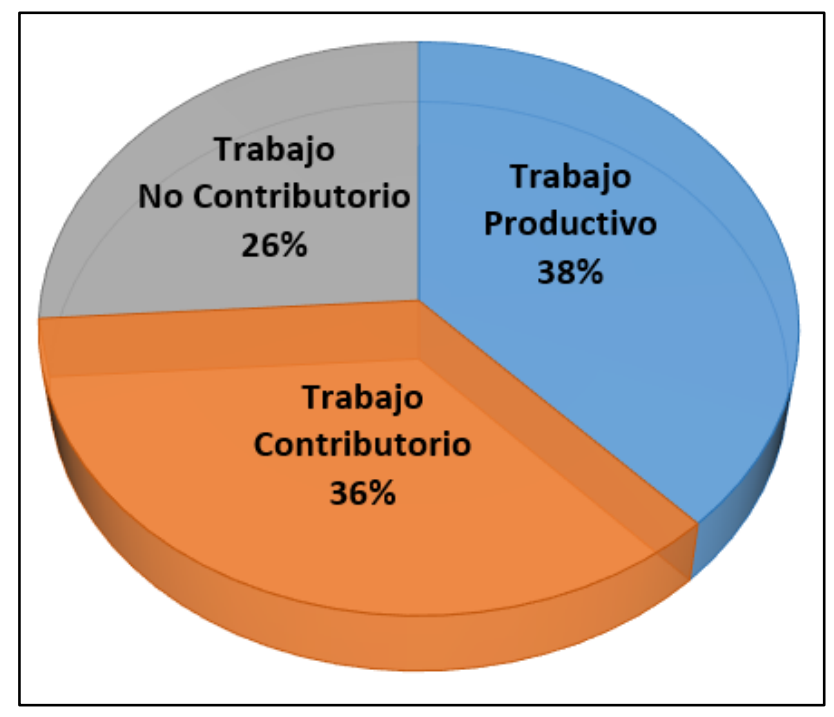

Mediante este análisis se observa que solo el 38\% de las actividades son productivas y el resto (62\%) constituye actividades improductivas y/o de soporte. Las siguientes actividades no contributorios son las que se identificaron en el presente estudio, ya que reducen el tiempo disponible para la ejecución de los trabajos productivos:

- Espera por instrucciones

- Espera y retiro de herramientas y materiales

- Solicitud y espera del equipo

- Espera por inspección

- Transporte innecesario

- Espera por espacio para trabajar

Estas actividades se producen debido a la falta de entrega de materiales, herramientas y equipos a las cuadrillas correspondientes para la ejecución de sus tareas y por los métodos de trabajo no son los apropiados para realizar las actividades programadas (Serpell, 2002).

Asimismo, Botero \& Álvarez (2003) muestra dos estudios, realizados en Chile y en Colombia, sobre la distribución de las categorías de trabajo y la identificación de pérdidas en la construcción. En el caso de Chile, el equipo de profesionales del departamento de Ingeniería Civil y Gestión de la Construcción de la Pontificia Universidad Católica de Chile realizó varias mediciones sobre las pérdidas en la construcción en más de 40 obras 
durante cinco años. Los siguientes resultados de la investigación en mención se observan a continuación en la Figura N8 y Figura N9.

Figura No 8: Distribución de categorías de trabajo en Chile (Botero \& Álvarez, 2003)

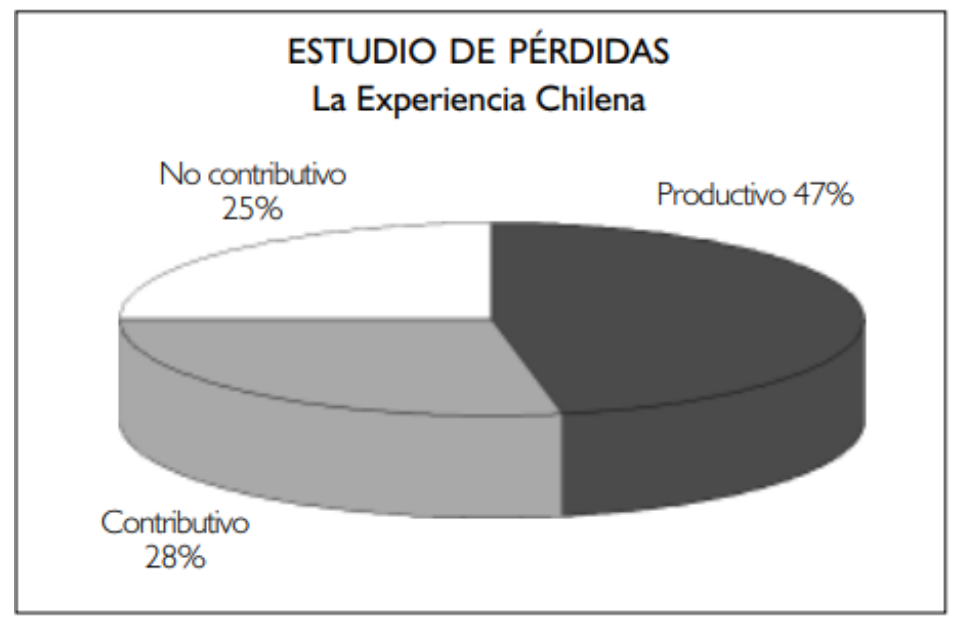

Figura No 9: Distribución del tiempo no contributivo en Chile (Botero \& Álvarez, 2003)

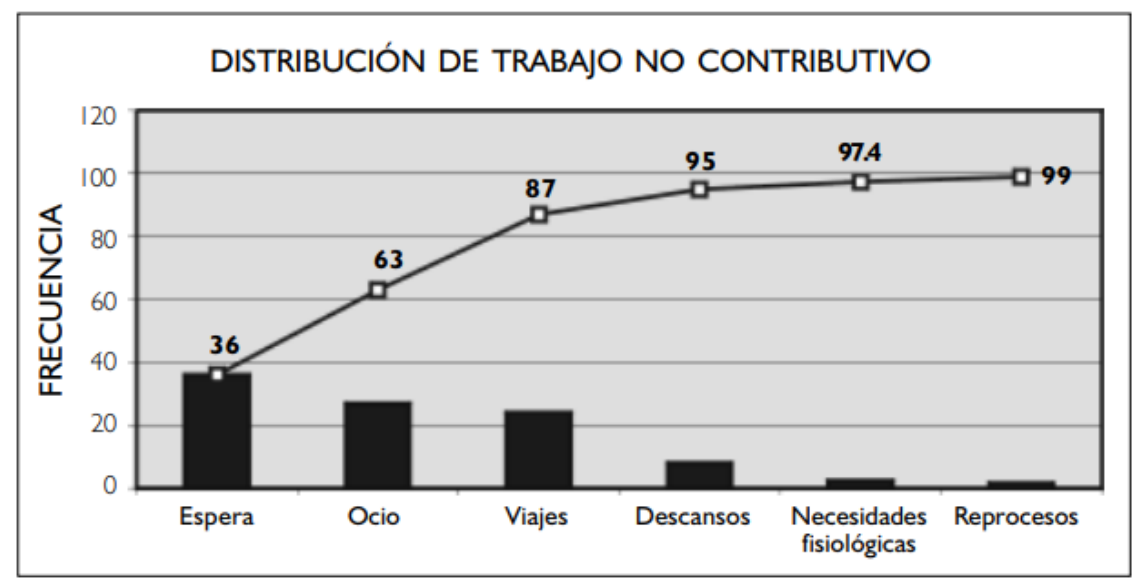

Como se aprecia en la Figura N7, Botero \& Álvarez (2003) concluyen que el 53\% del tiempo en la ejecución de los trabajos se realizan actividades improductivas. Asimismo, señalan que las causas que producen estás pérdidas se deben a la inadecuada planificación y programación de los recursos y actividades, lo cual se traduce en el exceso de esperas y viajes que perjudican al recurso humano.

Por otro lado, en el caso de Colombia, el área de construcción del departamento de Ingeniería Civil de la Universidad EAFIT, en conjunto con un grupo de constructores de la ciudad de Medellín, llevó a cabo el proyecto de mejoramiento de la productividad en la construcción. Este proyecto se realizó en tres diferentes proyectos de viviendas 
(aproximadamente $12000 \mathrm{~m} 2$ en total) y se obtuvieron los siguientes resultados, los cuales se observan en la Figura $\mathrm{N}^{\circ} 10$ y Figura $\mathrm{N}^{\circ} 11$.

Figura Nº 10: Distribución de categoría de trabajo - Colombia (Botero \& Álvarez, 2003)

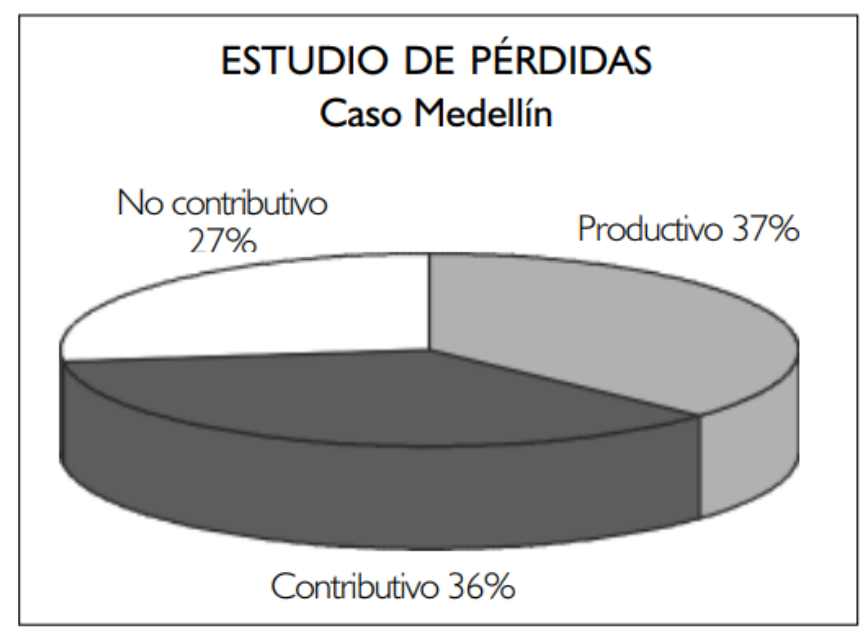

Figura No 11: Distribución del tiempo no contributivo - Colombia (Botero \& Álvarez, 2003)

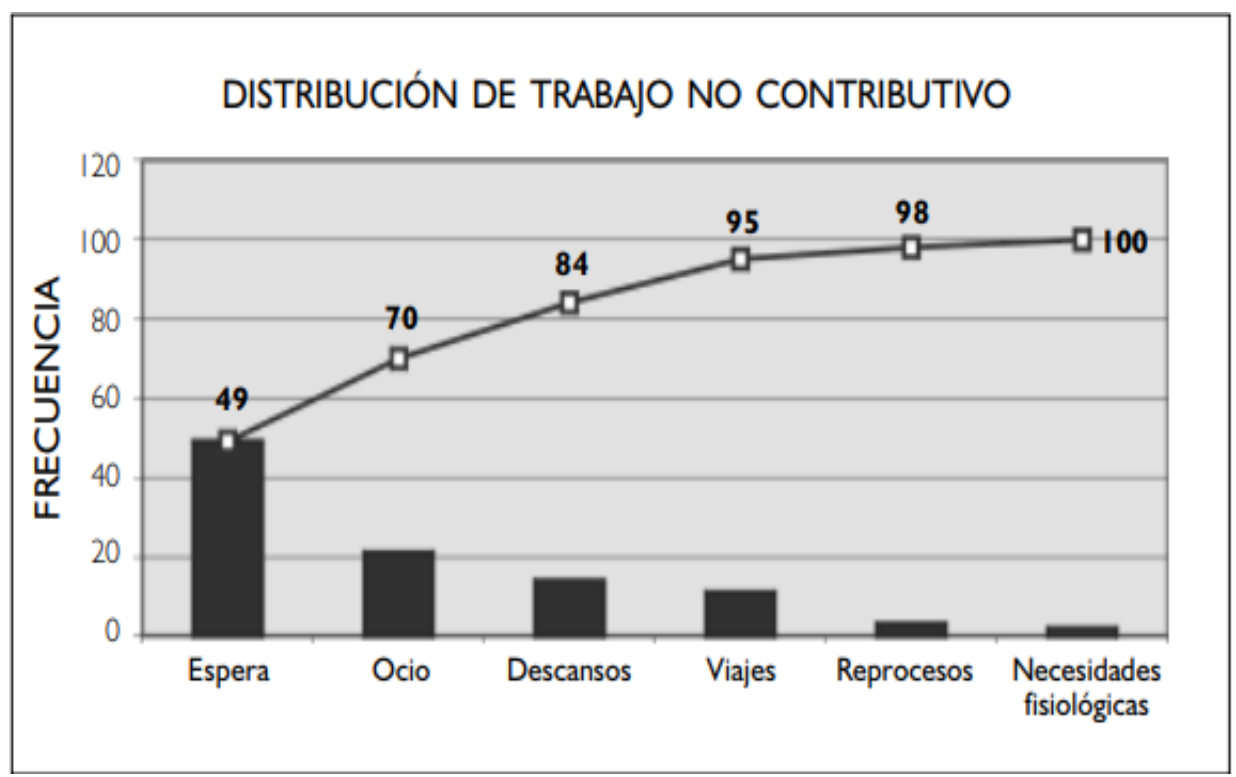

Según los resultados vistos en la Figura Nº9, en relación a la investigación en Chile, se mantiene un alto porcentaje de tiempo no productivo como promedio general (63\%). También, en la Figura $\mathrm{N}^{\circ} 11$ se observan las causas del trabajo no contributorio, que se centran principalmente en las esperas durante la ejecución de las actividades (49\%), por lo que los autores recomiendan, en este caso, tener una mejor planificación para la ubicación y disposición de los recursos, mejorar la gestión de la mano de obra, entre otras 
posibles mejoras para reducir el porcentaje de los trabajos improductivos para transformarlos a tiempos productivos.

Asimismo, en el Perú se realizaron investigaciones similares a las que se efectuaron en Chile y en Colombia con el objetivo de brindar un diagnóstico de los niveles de productividad en la construcción de nuestro país y determinar cuáles son las principales causas que generan las pérdidas o tiempo improductivo en la construcción. La primera investigación lo realizó el Ing. Virgilio Ghio en conjunto con un grupo de alumnos de la Pontificia Universidad Católica del Perú a principios del año 1999.

Para la investigación en mención se tomó como muestra 50 obras en Lima, principalmente del rubro de edificaciones, las cuales fueron clasificadas por el tipo de empresa, presupuesto, tipo de edificación y grado de supervisión. En este estudio se realizaron entrevistas a los profesionales responsables de la obra y al personal obrero para determinar el estado de las empresas constructoras en el Perú en relación a los sistemas de administración de la obra, puesto que es un detalle con mucha relevancia cuando se busca identificar las causas del tiempo improductivo y establecer las mejoras del caso. A continuación, se presenta los resultados obtenidos en la Tabla $\mathrm{N}^{\circ} 2$ y en la Figura $\mathrm{N}^{\circ} 12$.

Tabla Nº 2: Resultado de la distribución de categorías de trabajo en las 50 obras en Lima (Ghio, 2001)

\begin{tabular}{|l|c|c|c|}
\hline \multicolumn{1}{|c|}{ Valores } & $\begin{array}{c}\text { Trabajo } \\
\text { Productivo }\end{array}$ & $\begin{array}{c}\text { Trabajo } \\
\text { Contributorio }\end{array}$ & $\begin{array}{c}\text { Trabajo } \\
\text { No Contributorio }\end{array}$ \\
\hline Promedio - LIMA & $28.0 \%$ & $36.0 \%$ & $36.0 \%$ \\
\hline Mínimo & $20.0 \%$ & $35.0 \%$ & $45.0 \%$ \\
\hline Máximo & $37.0 \%$ & $36.0 \%$ & $26.0 \%$ \\
\hline
\end{tabular}


Figura Nº 12: Distribución de categorías de trabajo en Perú (Ghio, 2001)

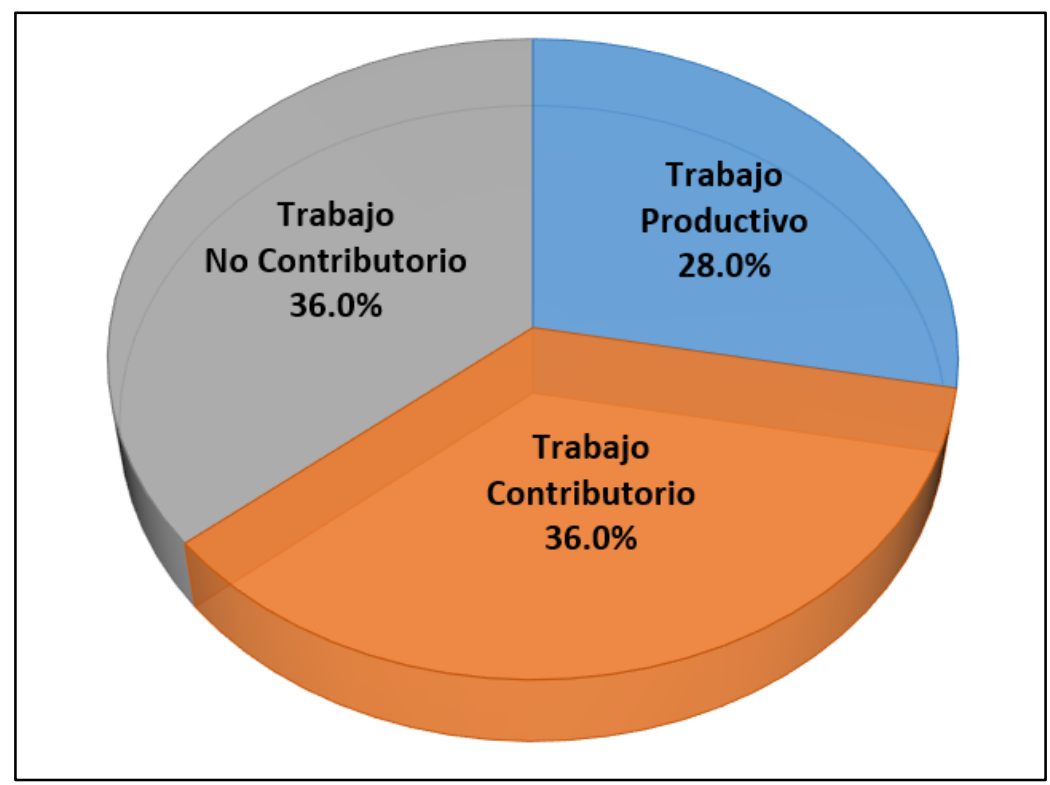

Según los resultados mostrados, el trabajo productivo tiene un porcentaje promedio del $28 \%$, es decir, que del $100 \%$ del tiempo solo se emplea el $28 \%$ de la mano de obra en actividades productivas. Este valor está muy por debajo de los niveles óptimos que se generan cuando una obra de construcción presenta un aumento de la productividad. Asimismo, se está invirtiendo aproximadamente un $75 \%$ de recursos y tiempo en actividades que no son productivas, las cuales son generadas por diversos factores relacionados a la gestión de operaciones de construcción y a la motivación del recurso humano.

El Ing. Virgilio Ghio señala las principales pérdidas en los procesos de producción que logró identificar mediante el estudio realizado. En la siguiente Tabla N³ se mostrará los principales factores que influyen en el aumento del trabajo contributorio y no contributorio. 
Tabla N 3: Principales causas de pérdidas de la construcción en el Perú (Ghio, 2001)

\begin{tabular}{|c|c|c|c|}
\hline $\begin{array}{c}\text { Categoria de } \\
\text { Trabajo }\end{array}$ & $\begin{array}{l}\text { Categoria de } \\
\text { Pérdida }\end{array}$ & $\begin{array}{c}\% \text { de } \\
\text { influencia }\end{array}$ & Causas más frecuentes \\
\hline \multirow{14}{*}{$\begin{array}{c}\text { Trabajo } \\
\text { No } \\
\text { Contributorio }\end{array}$} & \multirow{4}{*}{ Viajes } & \multirow{4}{*}{$13.0 \%$} & Cuadrillas sobredimensionadas \\
\hline & & & Falta de supervisión \\
\hline & & & Deficiencias en el flujo de materiales \\
\hline & & & Mala distribución de instalaciones en obra \\
\hline & \multirow{3}{*}{ Tiempo Ocioso } & \multirow{3}{*}{$10.0 \%$} & Falta de supervisión \\
\hline & & & Cuadrillas sobredimensionadas \\
\hline & & & Actitud del trabajador \\
\hline & \multirow{3}{*}{ Esperas } & \multirow{3}{*}{$6.00 \%$} & Cuadrillas sobredimensionadas \\
\hline & & & Falta de campo \\
\hline & & & Deficiencias en el flujo de materiales \\
\hline & \multirow{4}{*}{ Trabajo rehecho } & \multirow{4}{*}{$3.00 \%$} & Mala calidad \\
\hline & & & Trabajos mal ejecutados \\
\hline & & & Deterioros de trabajos ya realizados \\
\hline & & & Cambios en los diseños \\
\hline \multirow{4}{*}{$\begin{array}{c}\text { Trabajo } \\
\text { Contributorio }\end{array}$} & \multirow{2}{*}{$\begin{array}{c}\text { Transporte } \\
\text { Manual }\end{array}$} & \multirow{2}{*}{$14.0 \%$} & Deficiencias en el flujo de materiales \\
\hline & & & Falta de programación y control del uso de equipos \\
\hline & \multirow{2}{*}{ Otros } & \multirow{2}{*}{$11.0 \%$} & Trabajos lentos \\
\hline & & & Falta de diseño de los procesos constructivos \\
\hline
\end{tabular}

Como se muestra en la Tabla $N^{\circ} 3$, se puede concluir que un $27 \%$ del tiempo del trabajo ejecutado por la mano de obra se emplea principalmente al transporte (14\%) y a los viajes (13\%). Es decir, se invierte lo mismo en actividades productivas que en la movilización de los obreros. Cabe resaltar que las esperas (6\%) y el tiempo ocioso (10\%) presentan valores que tienen un cierto grado de influencia en los trabajos no contributorios. A continuación, se darán diversos ejemplos sobre las principales causas de pérdidas más influyentes señaladas en la Tabla $\mathrm{N}^{\circ} 3$.

a) Cuadrillas sobredimensionadas

- Exceso de personal obrero en el proyecto cuando no existen frentes de trabajo disponibles y deben realizar actividades auxiliares, como limpieza.

- Falta de conocimiento acerca los recursos necesarios para cada actividad, por lo generalmente se emplea mayor mano de obra del necesario.

b) Falta de supervisión 
- $\quad$ No se responsabiliza al personal por el cumplimiento de los trabajos programados y se termina generando intervalos de inactividad.

- Exceso de desperdicio de materiales o de viajes dentro de obra innecesarios.

c) Deficiencias en el flujo de materiales

- Ineficiente gestión del abastecimiento de los materiales, lo que genera que los obreros busquen sus materiales abandonando sus actividades.

- Inadecuada ubicación de los almacenes de obra, lo que produce que existan viajes o movimientos largos e innecesarios para pedir materiales y/o herramientas.

- Falta de uso de equipos de carga para movilizar materiales, en lugar de estar empleando la mano de obra en transportes complicados e innecesarios.

- Incorrecta gestión de la logística en la obra para el abastecimiento de materiales por parte de proveedores.

d) Mala distribución de instalaciones en obra

- Accesos obstaculizados que dificultan la movilización.

- Incorrecta ubicación de los servicios higiénicos durante la ejecución de las actividades.

e) Falta de programación y control en el uso de equipos

- Inadecuada programación para el empleo de equipos de transporte de materiales.

- Falta de una programación de mantenimiento de equipos. El mantenimiento de los equipos es reactivo, es decir, se realiza cuando se produce la falla.

- Carencia de equipos necesarios para agilizar diversas actividades en la obra.

Con estas pérdidas identificadas y sus causas más frecuentes, el autor concluye que gran parte de las fuentes de pérdidas tienen que ver directamente con los sistemas de administración de la producción. Por este motivo, cabe recalcar, el autor señala que es necesario centrar los esfuerzos a la reducción de las actividades no productivas mencionadas. Esto se puede lograr mediante mejoras en los sistemas de producción, mejor supervisión, planificación más eficiente, entre otros, teniendo en cuenta una constante evaluación de los cambios en la distribución del trabajo por medio de diversas herramientas para medir los índices de productividad.

Finalmente, Morales \& Galeas (2006) realizan una investigación para clasificar las actividades ejecutadas por los obreros con el fin de analizar los niveles de productividad 
en Lima y su evolución desde la investigación realizada por el Ing. Virgilio Ghio en 1999, la cual se tomará en cuenta para los criterios del estudio. La muestra de la investigación es de 26 obras y se empleó la carta balance para las mediciones correspondientes, en donde se identificaba 400 actividades por cada medición. Asimismo, se realizaron entrevistas a todos los involucrados de las obras para que den su punto de vista sobre el desempeño de la gestión de la obra. A continuación, se presenta los resultados finales del estudio en mención en la Tabla $\mathrm{N}^{\circ} 4$ y en la Figura $\mathrm{N}^{\circ} 13$.

Tabla Nº 4: Porcentaje de tiempo empleado por actividad. (Morales \& Galeas, 2006)

\begin{tabular}{|l|l|r|}
\hline \multicolumn{2}{|c|}{ Actividad } & \multicolumn{1}{c|}{$\%$} \\
\hline \multirow{3}{*}{ TP } & Trabajo Productivo & $22.40 \%$ \\
\cline { 2 - 3 } & Habilitación de materiales & $9.10 \%$ \\
\hline \multirow{4}{*}{ TC } & Transporte de todo & $18.80 \%$ \\
\cline { 2 - 3 } & Limpieza de todo & $4.20 \%$ \\
\cline { 2 - 3 } & Dar y recibir instrucciones & $6.00 \%$ \\
\cline { 2 - 3 } & Mediciones & $5.80 \%$ \\
\cline { 2 - 3 } & Otros & $8.20 \%$ \\
\hline \multirow{4}{*}{ TNC } & Viajes & $12.90 \%$ \\
\cline { 2 - 3 } & Esperas & $7.60 \%$ \\
\cline { 2 - 3 } & Tiempo Ocioso & $2.60 \%$ \\
\cline { 2 - 3 } & Trabajo Rehecho & $0.70 \%$ \\
\cline { 2 - 3 } & Otros & $1.70 \%$ \\
\hline
\end{tabular}

Figura N 13: Distribución de categorías de trabajo en Perú (Morales \& Galeas, 2006)

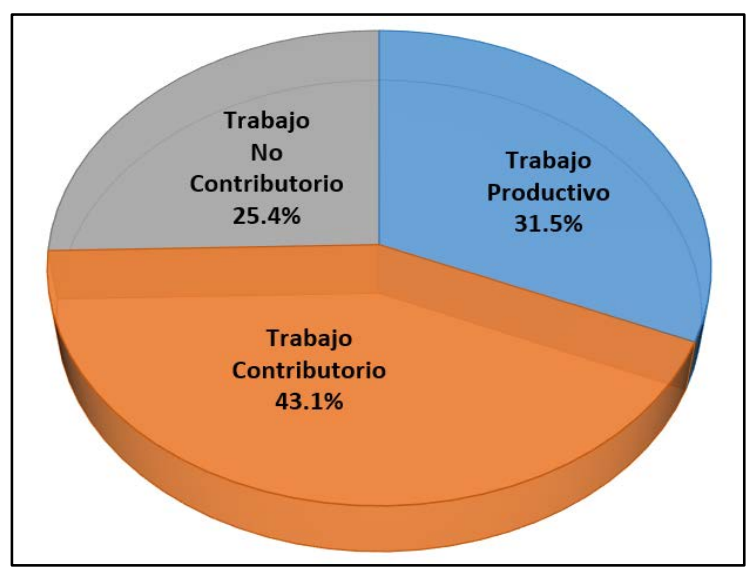

Según los resultados de la Tabla $\mathrm{N}^{\circ} 4$, se observa que las actividades no productivas (tanto contributorio como no contributorio) que tienen mayor incidencia son el Transporte (18.80\%) y los Viajes (12.90\%), lo cual tiene bastante similitud con los resultados expuestos de las otras investigaciones en el presente capítulo. Por otro lado, como se 
muestra en la Figura $\mathrm{N}^{\circ} 13$, existe una disminución del trabajo no contributorio entre las investigaciones de 1999 y del 2006, sin embargo, el trabajo contributorio aumentó cerca de $8.00 \%$, lo que significa que se aumentaron los trabajos de apoyo, como por ejemplo el transporte.

Estos resultados en el trabajo no contributorio y contributorio demuestran que el porcentaje de actividades no productivas no ha disminuido significativamente desde de 1999 hasta el 2006, puesto que se acerca a un 70\%. Además, la Figura №13 muestra que el porcentaje del trabajo productivo es de 31.5\%, lo que significa que los índices de productividad no han tenido un incremento relevante desde la investigación de 1999 por parte del Ing. Virgilio Ghio. Esto significa que a pesar de contar con metodologías o herramientas para aumentar la productividad en obra, existen varias obras cuyo nivel de gestión es inadecuado, debido a que se presenta diversos problemas en los siguientes aspectos (Morales \& Galeas, 2006):

- Diseño de procedimientos de construcción

- Transmisión de la planificación

- Equipo encargado de la planificación

- Planificación y distribución de la mano de obra

- Control y Capacitación

\subsubsection{HerRamienta de MEdición de Trabajo: CARTA BALANCE}

Esta herramienta nos ayuda a identificar con mayor claridad los tiempos productivos (TP), tiempos contributorios (TC) y tiempos no contributorios (TNC) de una actividad específica en la construcción, ya que para la mejora de la producción en la construcción, en específico en las partidas cuales quiera no es necesario presionar y exigir al personal que cumplan con su trabajo con mayor velocidad, si no es necesario reducir o eliminar los procesos que no aportan valor y que no son parte de la actividad que se tiene que realizar.

El proceso de la recopilación de datos con esta herramienta es la siguiente:

- Identificar los procesos que involucran la actividad específica.

- Agrupar los procesos en TP, TC y TNC. 
- Se realiza el muestreo de las actividades por cada obrero con una duración de un minuto.

- La medición se realiza por un tiempo considerable, recomendable por 100 -120 minutos.

- Finalmente se realiza un análisis estadístico donde se mostrará la cantidad de TP, TC y TNC en cada una de las actividades.

\subsection{Estrategias Para mejorar la Productividad}

Teniendo en cuenta las investigaciones mencionadas en el presente capítulo, en el sector de la construcción existen varios factores que impiden ejecutar las obras de la manera más eficiente y eficaz posible.

Estos estudios presentan resultados que indican que, en la mayoría de obras, solo una tercera parte del tiempo se está ejecutando actividades productivas y el resto del tiempo se emplea en actividades no productivas, debido a los diversos problemas presentados que afectan la productividad en la construcción. Esto produce una gran cantidad de “pérdidas” que afectan directamente al presupuesto y al cronograma de la obra.

Por lo tanto, se presentará diversas estrategias para aumentar los índices de productividad en las obras de construcción, logrando implementar acciones correctivas y preventivas ante los problemas expuestos anteriormente. Cabe señalar que para la implementación de una estrategia de mejoramiento se de considerar los siguientes propósitos (Mejía \& Hernández, 2007):

- Mejorar el desempeño del proceso identificado y definir sus actividades, lo que se traduce en ser más efectivos.

- Mejorar el uso de los recursos activos de la obra, como la mano de obra y los equipos. Es decir, ser más eficiente.

- Mejorar la calidad del proceso, priorizando que el resultado final agregue valor al producto que se entregará a la otra parte.

- Mejorar las condiciones laborales para motivar a la mano de obra a ser más productivo. 
- Tomar en cuenta las lecciones aprendidas y el mejoramiento continuo, de manera que se aproveche la curva de aprendizaje.

Se hace mención a la mejora de los procesos, puesto que mejorar la productividad implica principalmente mejorar los procesos con los que se está trabajando en el proyecto. Además, es fundamental considerar las mediciones dentro de toda estrategia de mejoramiento de la productividad, puesto que sirve para analizar los niveles reales de la productividad en la obra (Mejía \& Hernández, 2007).

\subsubsection{Ciclo de Mejoramiento de la Productividad}

El ciclo de mejoramiento de la productividad es una estrategia que se divide en tres etapas (Serpell, 2002), las cuales se muestran en la siguiente Figura $\mathrm{N}^{\circ} 14$ :

Figura No 14: Ciclo de mejoramiento de la productividad (Serpell, 2002)

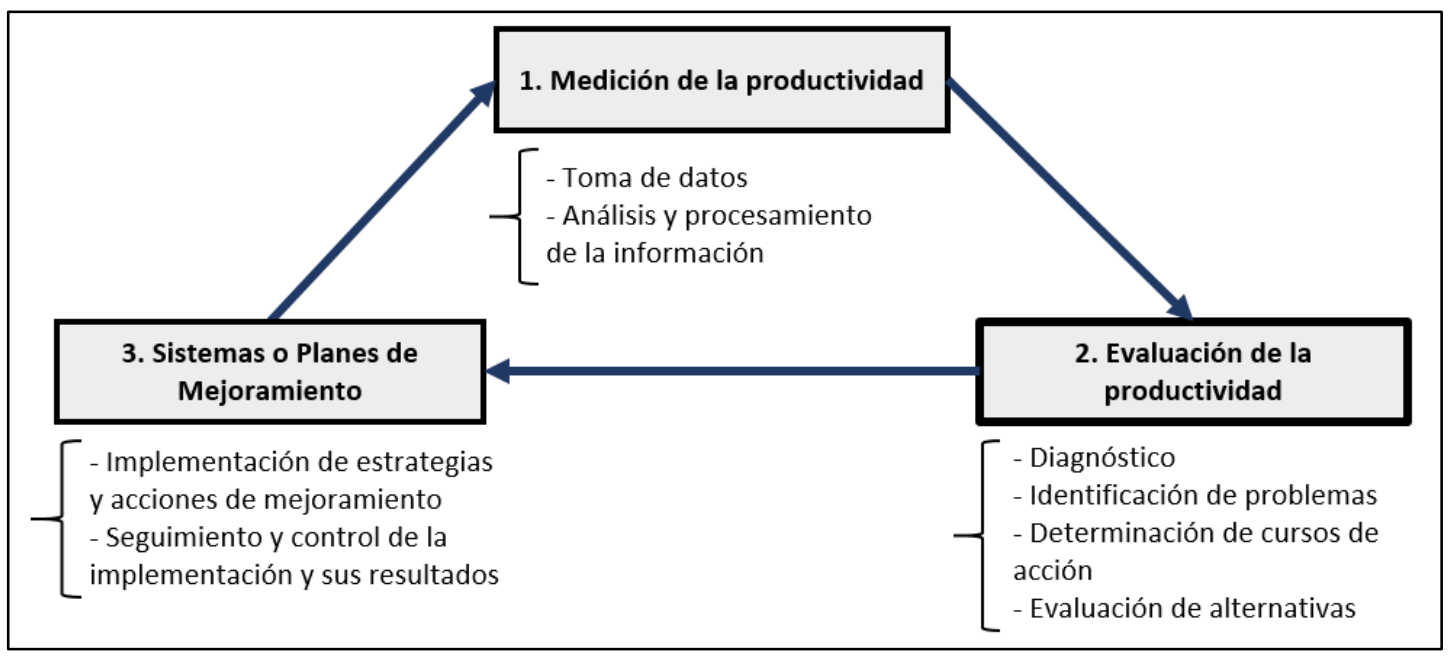

Como se comentó anteriormente, la medición es la base fundamental para el inicio del mejoramiento de la productividad en las obras. La medición de la productividad debe realizarse de la manera más transparente posible puesto que, si se le toma la importancia del caso, es una gran herramienta para determinar los puntos de mejora dentro de los procesos que se están ejecutando. Los objetivos de la etapa de medición de la productividad son los siguientes (Serpell, 2002) :

- Evaluar el desempeño, de manera transparente, de las actividades que se están ejecutando (generalmente, las que presentan mayores costos)

- Identificar los problemas que existen durante la ejecución de las actividades. 
- Identificar las diferencias que existen entre actividades para establecer las razones que generan que una sea más productiva que otra.

- Realizar análisis de tendencia y pronósticos de costo y plazo en base al estado actual de los índices de productividad medidos.

La manera de medir la productividad en obras a nivel general consiste en, primero, determinar las actividades en ejecución a analizar. Segundo, realizar los informes diarios de las horas hombre empleadas para la ejecución de la actividad en mención y la cantidad que se realizó durante el día. En función a las horas hombre y la cantidad ejecutada se procede a calcular la productividad. Finalmente, se procede a realizar un análisis de tendencias, una evaluación de desempeño y el pronóstico de las horas hombre totales a emplear. Estos resultados finales brindan la información necesaria para determinar si las actividades evaluadas se ejecutan de manera productiva o improductiva (Serpell, 2002).

Cabe señalar que la forma más representativa de mostrar los resultados de las mediciones de productividad es por medio de los gráficos circulares, en donde se muestra la distribución de las categorías de trabajo de la obra analizada (la Figura $N^{\circ} 5$ sirve como referencia).

Después de obtener todos los resultados correspondientes a la medición de la productividad, se procede a la etapa de evaluación de la productividad. En esta etapa, se hace un diagnóstico de la situación actual y se identifica los problemas que impiden aumentar la productividad de las actividades. Una vez que se tiene los problemas identificados, se procede a evaluar las diversas estrategias o acciones de mejoramiento y se determina la que pueda reducir las pérdidas de trabajo productivo de manera más eficiente y efectiva (Serpell, 2002).

Finalmente, la etapa de sistemas de mejoramiento consiste en la implementación de las estrategias o acciones de mejoramiento seleccionadas en la etapa previa. En la implementación se debe contar con el apoyo de todos los involucrados para evitar inconvenientes durante la ejecución de estas estrategias. Asimismo, se realiza un seguimiento y control de la implementación y se analiza los resultados que se obtiene de la estrategia. Si no hay buenos resultados o existe posibilidad de mejorar, se vuelve a 
medir la productividad dando inicio de nuevo al ciclo de mejoramiento de la productividad (Serpell, 2002).

\subsubsection{ESTUDIO DE TRABAJO}

Esta estrategia consiste en analizar y procesar la información para hacer seguimiento a la productividad, en base a mediciones. Para esto se emplea las dos técnicas: el análisis de métodos y el estudio del tiempo (Mejía \& Hernández, 2007)

El análisis de métodos es un método que identifica y analiza los procedimientos constructivos empleados y, a su vez, está en la constante búsqueda de un procedimiento más práctico, eficiente, efectivo y productivo que el procedimiento registrado. Es decir, se busca de manera constante efectuar mejoras en los procedimientos que se ejecutan durante la construcción.

Existen diversas situaciones que se pueden mejorar al utilizar el análisis de métodos como, por ejemplo, existencia de cuellos de botella en el flujo de materiales, exceso de desperdicios de materiales, recurrentes errores en la ejecución, inadecuadas condiciones de trabajo, incorrecta distribución de los almacenes de obra, retrasos en actividades por subcontratistas, entre otros (Harris \& McCaffer, 2005).

Los pasos a seguir para ejecutar el análisis de métodos consisten en identificar y presentar los problemas que presentan uno o varios procesos, realizar el análisis de la información, desarrollar e implementar el proceso ideal, establecer un procedimiento de trabajo con los estándares correspondientes para capacitar al personal a cargo de la implementación y, finalmente, efectuar un seguimiento y control del proceso implementado. De esta manera, se logra eliminar o reducir los problemas como los mencionados anteriormente (Harris \& McCaffer, 2005). Este procedimiento se presenta en forma de esquema en la Figura $\mathrm{N}^{\circ} 15$. 
Figura Nº 15: Procedimiento del Análisis de Métodos (Harris \& McCaffer, 2005)

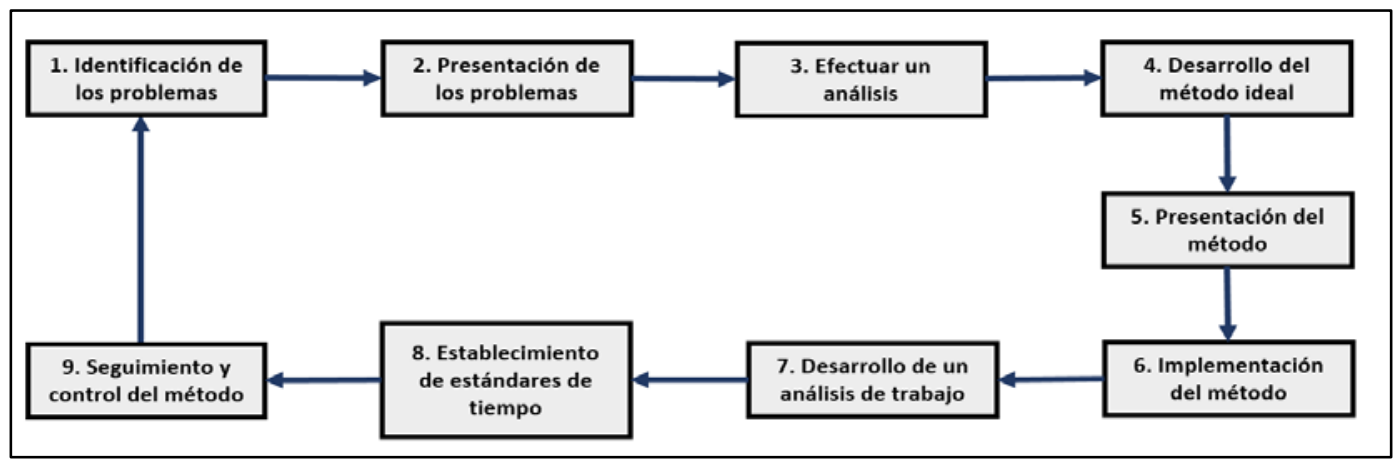

Por otro lado, el estudio de tiempos es un método que identifica, analiza y determina el tiempo necesario para ejecutar una actividad bajo los rendimientos preestablecidos que se manejan en la obra. El tiempo en este estudio representa los rendimientos de las cuadrillas de trabajo en función a un alcance definido, el cual se puede medir por medio de dos técnicas (Mejía \& Hernández, 2007):

- Observación directa: Medir tiempos reales mientras se observa la ejecución de las actividades. Estos tiempos se pueden estimar de manera aleatoria, como los muestreos de trabajo.

- Tiempos predeterminados: Establecer tiempos determinados para ejecutar los trabajos que componen una actividad con el objetivo de establecer el tiempo total que se tiene que emplear para la ejecución de esta actividad.

Uno de los objetivos del estudio de tiempos es establecer tiempos fijos de desempeños tipo (rendimientos obtenidos de forma natural) como base para el seguimiento y mejoramiento de las actividades. Asimismo, sirve para determinar registros históricos de rendimientos que pueden ser utilizados para otros proyectos de construcción (Mejía \& Hernández, 2007). Los resultados obtenidos de la medición del tiempo se pueden emplear en diversos aspectos como, por ejemplo, en la determinación de la cantidad de mano de obra o equipos a utilizar en las actividades, en la implementación de sistemas de incentivos financieros, en el control de costos y tiempos del proyecto y en la determinación de procesos más efectivos (Harris \& McCaffer, 2005).

El estudio de tiempos toma en cuenta las habilidades y actitudes del trabajador, las condiciones laborales, el estado de los materiales y equipos a emplear, entre otros. 
Teniendo en cuenta esto, para realizar el estudio de tiempos es necesario conocer las siguientes consideraciones (Harris \& McCaffer, 2005):

- Utilización de los equipos básicos de medición: consisten en un cronómetro, una tabla, un formato preparado con la cantidad de información necesaria para la medición, una cinta métrica, una calculadora, entre otras dependiendo del nivel de detalle de la medición.

- Explicación del estudio: se debe explicar a los trabajadores y al encargado del grupo sobre la medición a realizar, de manera que no se incomoden al momento de estar realizando la medición mientras ejecutan sus tareas.

Según lo descrito, el estudio de trabajo y el estudio de tiempos son métodos de gran ayuda para mejorar la productividad en las obras, ya que se basan principalmente en cuatro aspectos que son la medición del trabajo (cálculo de la productividad actual, identificación de problemas), análisis de resultados (determinar si el proceso actual cumple con lo solicitado, búsqueda de procesos más efectivos), a implementación del proceso ideal (capacitación al personal sobre el nuevo proceso) y el seguimiento y control del proceso implementado. Teniendo en cuenta estos aspectos, se puede lograr ejecutar una obra de construcción de una manera más eficiente y efectiva, eliminando la mayor cantidad de pérdidas posibles.

\subsubsection{LAST Planner System}

El sistema Last Planner es una metodología que involucra a las etapas de planificación y control, cuyas principales intenciones son la reducción de la variabilidad en el trabajo y el aumento de la fiabilidad de la planificación, para conseguir, de esta manera, el aumento de la productividad en obra. El sistema Last Planner consiste básicamente en tres principios (Pellicer, Cerveró, Lozano, \& Ponz, 2015):

- Coordinación entre los últimos planificadores (los que deciden en obra) por medio de reuniones semanales.

- Compromiso de los involucrados en estas reuniones.

- Publicación de los resultados obtenidos durante la semana, por medio del indicador de control que se denomina Porcentaje de Plan Cumplido (PPC). 
Por lo descrito anteriormente, esta metodología es de gran ayuda durante la ejecución de la construcción, ya que se centra en el control continuo de las actividades planificadas, así como la mejora continua y retroalimentación de los trabajos ejecutados semana tras semana. Los principales objetivos del sistema Last Planner son los siguientes (Abdul, Vamsi, \& Ranjith, 2014):

- Reducir la variabilidad.

- Revisar las actividades completadas.

- Investigar y eliminar las causas de no cumplimiento de las actividades previstas para facilitar su integración en la programación semanal.

- Realizar una lista de las actividades que están preparadas para ser ejecutadas, con el fin de evitar inconvenientes en la ejecución de estas.

- Preparar las condiciones adecuadas para ejecutar una actividad sin ningún inconveniente, es decir, los requisitos previos deben ser "make-ready”.

- Agregar o cambiar las técnicas Pull en el método tradicional Push.

- Integrar a los involucrados de la ejecución del proyecto en la toma de decisiones, para así fomentar el trabajo colaborativo o concurrente.

El sistema Last Planner consiste en cinco elementos integrados. Estos componentes ofrecen varias ventajas y aumentan los beneficios en la planificación y control de proyectos, siempre que estos se implementen de manera sistemática en la práctica (Hamzeh, Ballard, \& Tommelein, 2012).

En la Figura $N^{\circ} 16$ se muestra los cinco elementos mencionados del sistema Last Planner con las actividades básicas que los componen y qué es lo que significa cada uno dentro de este sistema 
Figura No 16: Esquema del Sistema Last Planner (Rodriguez, Alarcón, \& Pellicer, 2013)

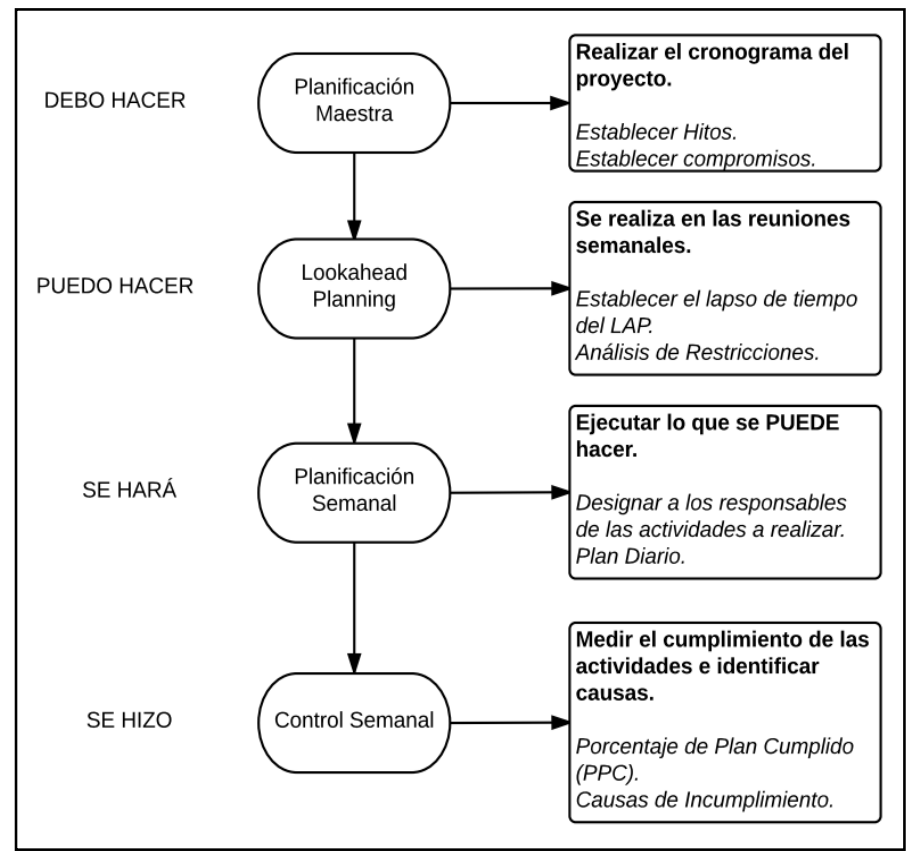

En la Tabla $\mathrm{N}^{\circ} 5$ se muestra las ventajas del sistema Last Planner con respecto al método tradicional, de esta forma se puede observar los cambios que se produjeron en el sistema Last Planner para mejorar el sistema tradicional.

Tabla N ${ }^{\circ}$ 5: Comparativo de Sistema Last Planner y Sistema Tradicional. (Kalsaas, Grindheim y Laeknes, 2014)

\begin{tabular}{|l|l|}
\hline \multicolumn{1}{|c|}{ Sistema Last Planner } & \multicolumn{1}{c}{ Sistema Tradicional } \\
\hline $\begin{array}{l}\text { Descentraliza decisiones para eliminar las restricciones y y } \\
\text { realiza el plan. }\end{array}$ & $\begin{array}{l}\text { No existe un enfoque sistemático en la eliminación de } \\
\text { restricciones. }\end{array}$ \\
\hline Control continuo. & El control se realiza "más tarde". \\
\hline $\begin{array}{l}\text { Mejora continua a causa de las Lecciones Aprendidas, } \\
\text { medición del PPC y análisis de causas. }\end{array}$ & $\begin{array}{l}\text { Reportes mensuales y las Lecciones Aprendidas se } \\
\text { evalúan cuando finaliza el proyecto. }\end{array}$ \\
\hline $\begin{array}{l}\text { Planifica las actividades por el método Pull y elimina } \\
\text { todas las restricciones en la construcción. }\end{array}$ & $\begin{array}{l}\text { Se utiliza el método de la Ruta Crítica para planificar } \\
\text { las actividades y no considera todas las restricciones. }\end{array}$ \\
\hline Técnicas de planificación y control simples y manuales. & $\begin{array}{l}\text { La planificación y control se realiza por una } \\
\text { computadora necesariamente. }\end{array}$ \\
\hline
\end{tabular}




\section{CAPÍTUlO III: METODOLOGÍA Y LEVANTAMIENTO DE INFORMACIÓN}

El presente capítulo tiene como objetivo dar a conocer la estrategia y la herramienta que fueron utilizadas para la metodología de la investigación, así como para el levantamiento de la información del presente caso de estudio. Con la información recolectada, se realizará su análisis para identificar el contexto de la obra y las consecuencias del bajo porcentaje de trabajo productivo en el caso de estudio.

\subsection{Estrategia: Ciclo de Mejoramiento de la Productividad}

Para la metodología de investigación de la presente tesis, se empleará el Ciclo de Mejoramiento de la Productividad (Serpell, 2002). Se utiliza esta estrategia de mejora de la productividad como metodología de investigación puesto que contiene los puntos necesarios para el correcto análisis de la situación actual del proyecto en relación a la productividad y para el desarrollo de una propuesta de mejoramiento.

El Ciclo de Mejoramiento de la Productividad se basa en tres etapas que son fundamentales para el desarrollo de la presente tesis, puesto que consiste en un paso a paso de cómo solucionar los problemas de productividad en las obras de construcción mediante un levantamiento y análisis de información, así como establecer un plan de mejoramiento para la productividad. A continuación, se presenta la manera en que se desarrollará las tres etapas del Ciclo de Mejoramiento de la Productividad en la presente tesis con el fin de lograr un correcto diagnóstico de la situación actual del caso de estudio y una propuesta que solucione directamente el problema.

1. Medición de la productividad: Se empleará la toma de datos mediante la Carta Balance, con el cual se obtendrá los gráficos de productividad.

2. Evaluación de la productividad: Con los resultados de la Carta Balance, se procederá con el diagnóstico de los problemas y la evaluación de las alternativas de mejora.

3. Plan de Mejoramiento: Se verificará cuáles de las alternativas propuestas son las adecuadas para solucionar los problemas identificados en la etapa anterior y se 
planifica la implementación de esta propuesta. Asimismo, se tiene en cuenta los indicadores a utilizar para medir su performance en el tiempo.

\subsection{Herramienta: Carta Balance}

Como se mencionó antes la herramienta para la recolección de datos será la carta balance, se detalló las características de este en el marco teórico. La metodología de uso en esta investigación será de la siguiente manera:

- La duración de cada medición será aproximadamente de 60 a 90 minutos (en las jornadas de la mañana y la tarde).

- El número de mediciones serán hasta obtener datos estables de cada partida.

- El personal de obra no será informado acerca de las mediciones.

- El personal encargado de la medición no debe interrumpir las labores del personal de obra, para ello se debe ubicar de manera estratégica además tener un panorama completo de la ejecución de la partida.

\subsection{El Proyecto}

\section{Proyecto: Edificio Residencial TWENTY}

Figura No 17: Edificio Residencial Twenty - HUBY Inmobiliaria
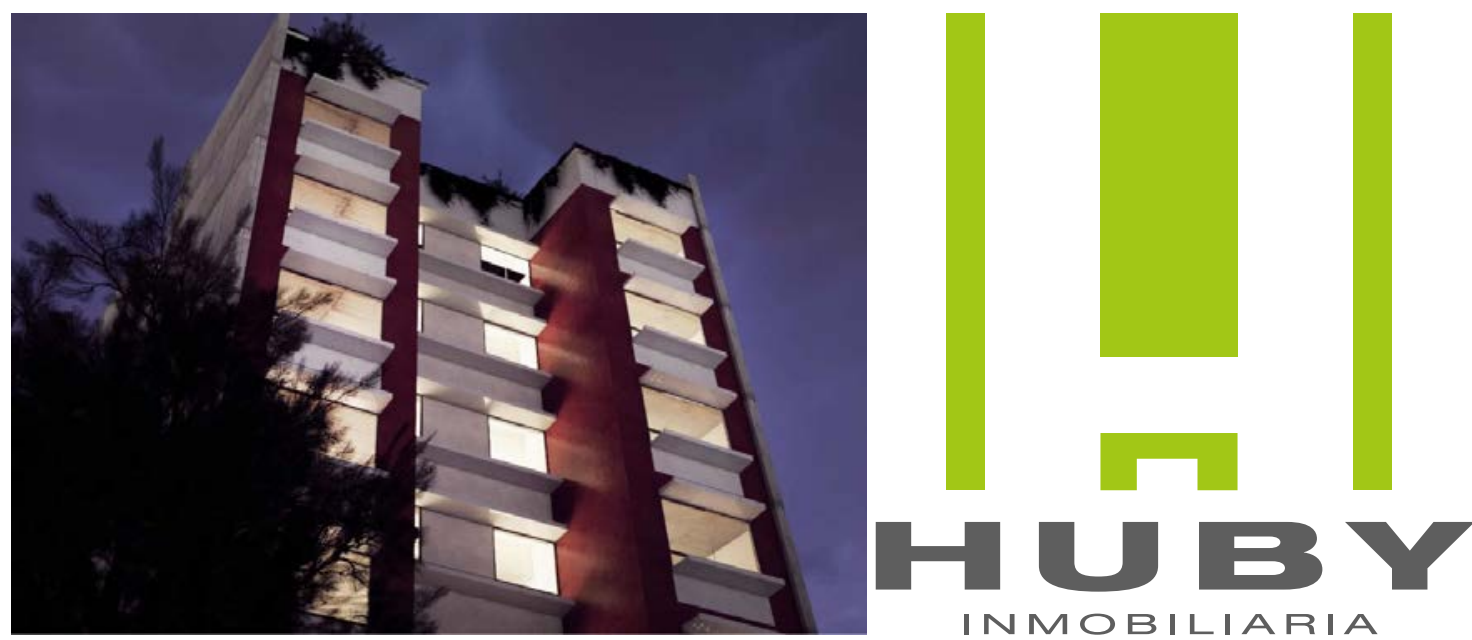

Fuente: HUBY Inmobiliaria 
Cliente: HUBY Inmobiliaria

Constructora: HUBY Inmobiliaria

Tipo de Edificación: Viviendas Multifamiliares

Presupuesto: S/. 5104484.50

Plazo de Ejecución: 15 de Enero del 2019 al 15 de Diciembre del 2019 (11 meses)

Costo de Construcción por m2: 510.00 dólares (incluido IGV)

Área del Terreno: $403 \mathrm{~m} 2$

$\underline{\mathrm{N}^{\circ} \text { de Pisos: }} 01$ Sótano, 01 Semisótano, 07 Pisos y 01 Azotea

$\underline{\mathrm{N}^{\circ} \text { de departamentos: }} 20$ departamentos (con áreas entre 78.0 m2 y 120.04 m2)

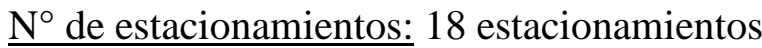

Áreas Comunes: Área de juego para niños, Sala de usos múltiples, Área de Parrillas.

\subsection{Descripción de las Partidas Analizadas en el Caso de ESTUDIO}

Antes de realizar el levantamiento de información mediante el uso de la Carta Balance, es necesario conocer los principales componentes que involucran las partidas que se analizarán en el caso de estudio. Para esto es necesario conocer el proceso constructivo de estas partidas y, de esta manera, se obtendrá todas las actividades que se necesitan para ejecutarlas. Conociendo estas actividades, se procede a la clasificarlas en las categorías de trabajo productivo y trabajo contributorio (no se clasifica en trabajo no contributorio puesto que esta categoría no contiene actividades que sirvan para la ejecución de la actividad). Con estos datos se puede comenzar a realizar el levantamiento de información mediante el uso de la Carta Balance.

Las partidas que se seleccionaron para la evaluación correspondiente son el Enchape de Cerámicos y el Asentado de Ladrillo Blanco. Se eligieron estás actividades puesto que son parte de la ruta crítica del proyecto y se encuentran en proceso de ejecución por el 
momento. A continuación, se presentará el proceso constructivo y los demás componentes que involucran las partidas a analizar en el Edificio Twenty.

\subsubsection{ENCHAPE DE CERÁMICOS}

a) Proceso Constructivo:

Antes de iniciar con los trabajos de enchape del cerámico, se debe verificar y seleccionar aquellas piezas que tengan el mismo tono, tamaño y serie. Asimismo, se debe verificar que el ambiente presente la geometría adecuada (plomadas, escuadras y niveles apropiados). Una vez que se tenga el ambiente liberado para enchapar, se realiza el trazado del enchape, así como la ubicación del arranque del mismo.

El trazado y el arranque deben estar validado por el arquitecto proyectista o el residente de obra. Antes de proceder con el enchapado, se debe proceder con la limpieza del ambiente para eliminar la tierra o polvo en el piso con el fin de que el pegamento adhiera correctamente el cerámico con el piso.

Seguido de esto, se coloca la primera hilada maestra, aplicando pasta sobre la superficie de manera uniforme con el raspín. Luego, se coloca las mayólicas en los extremos (como guía), de manera que coincida con la línea de referencia trazada. Las demás mayólicas se colocan de forma que coincida la arista superior con el cordel de alineación y se les golpea con el martillo de goma para que se asienten.

Una vez colocado la mayólica, se debe verificar que se encuentre nivelado con el resto de las mayólicas. Asimismo, se colocan crucetas entre mayólicas para que se formen las juntas para la fragua, cuyo espesor depende de lo establecido en los planos o por el arquitecto proyectista. Finalmente, se procede con la colocación de fragua en las juntas de los cerámicos y se hace limpieza de las piezas para su oportuna entrega.

A continuación, se presentan las siguientes imágenes sobre el proceso constructivo del enchape de cerámico: 
Fotografía No 1: Enchape de Cerámicos - Edificio Twenty.

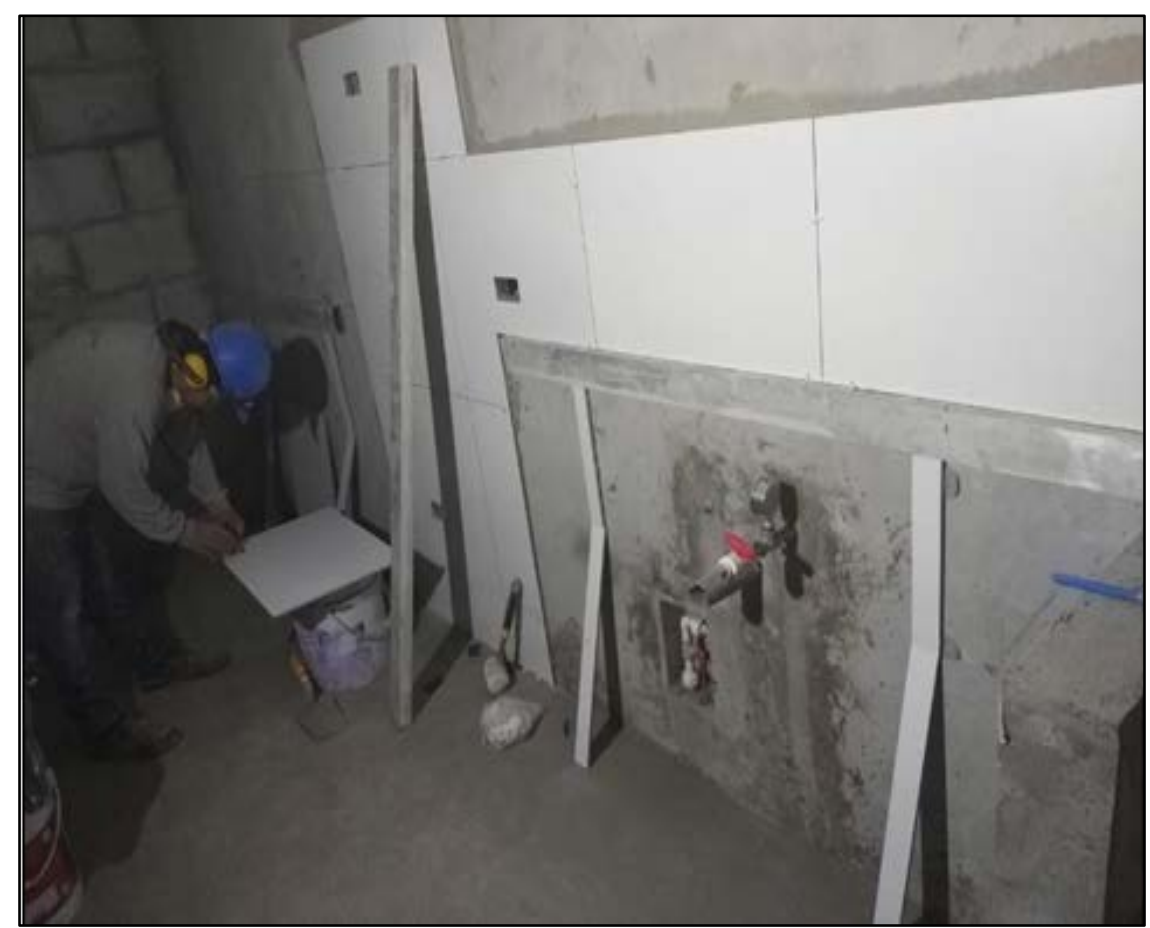

Fuente: Elaboración Propia.

Fotografía № 2Enchape de Cerámicos - Edificio Twenty.

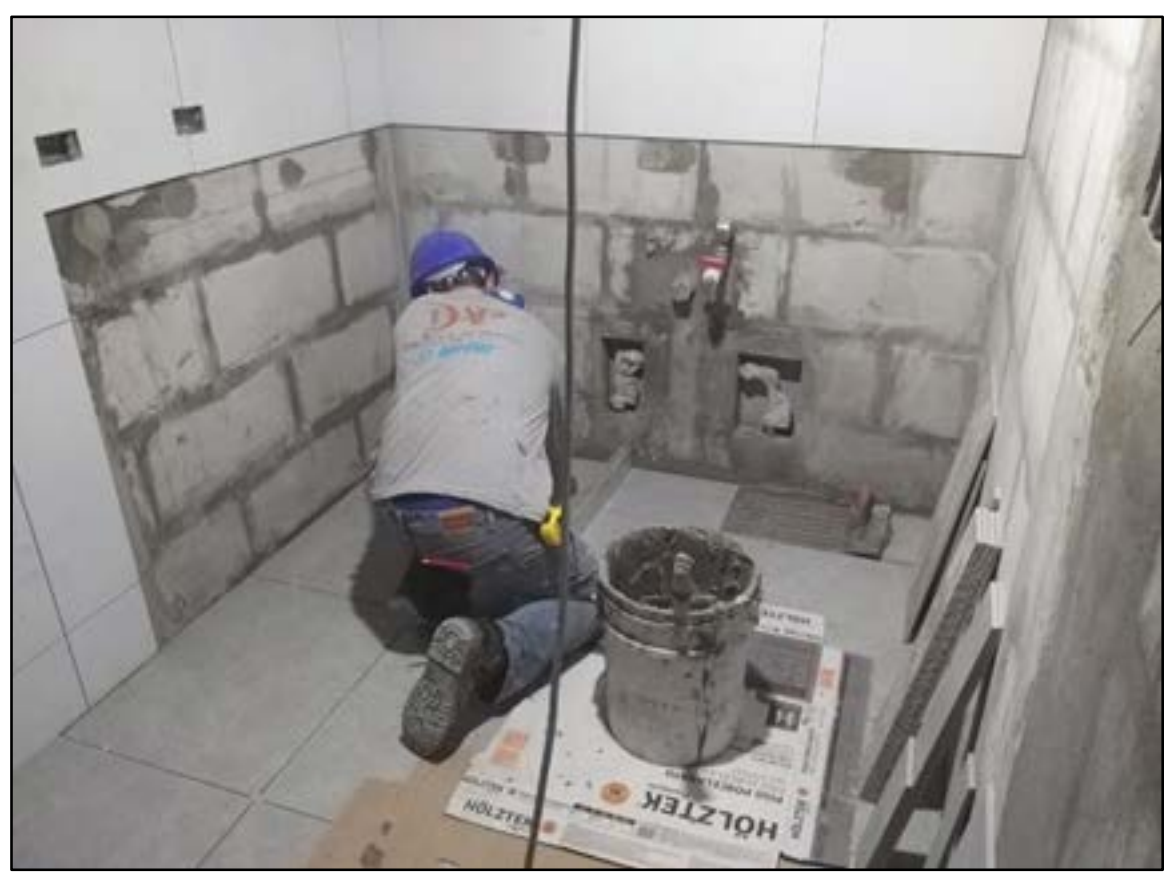

Fuente: Elaboración Propia. 
Fotografía No 3: Enchape de Cerámicos - Edificio Twenty.

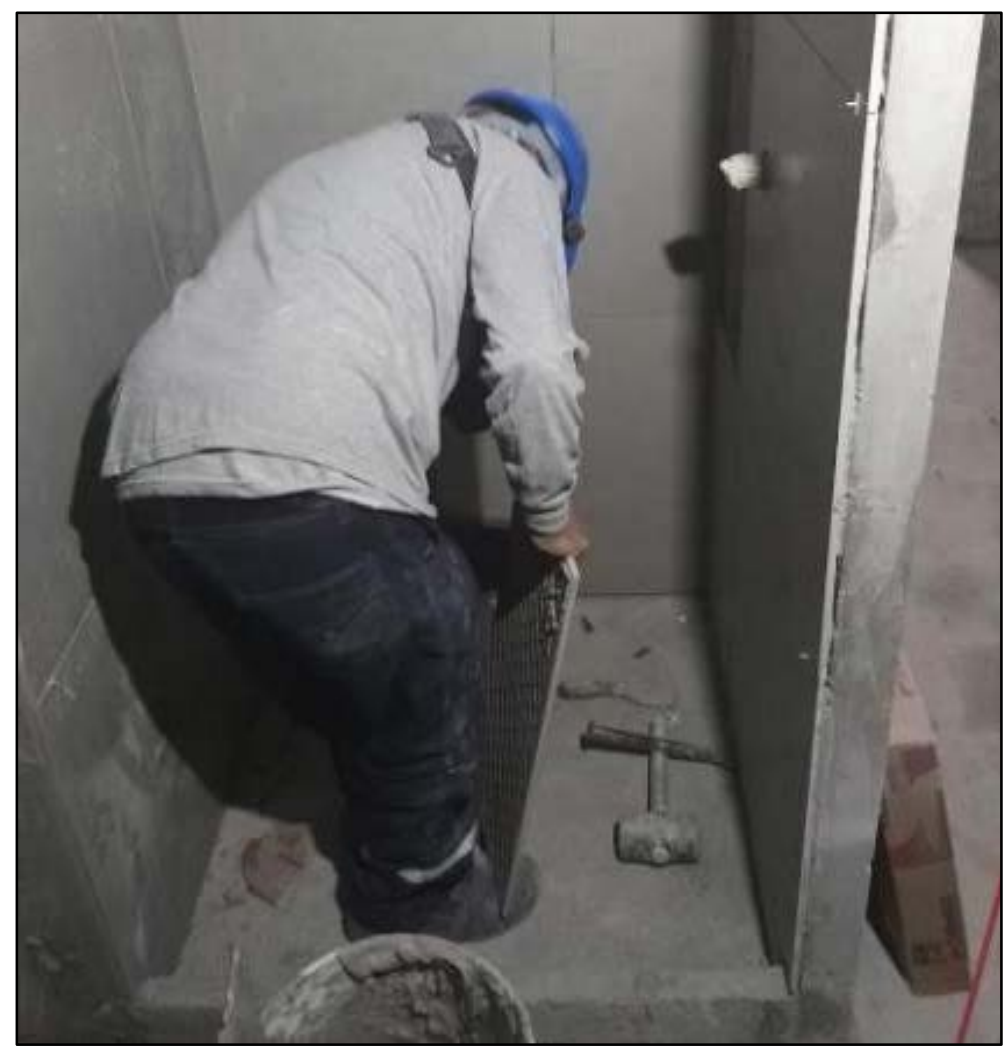

Fuente: Elaboración Propia.

Fotografía № 4: Enchape de Cerámicos - Edificio Twenty.

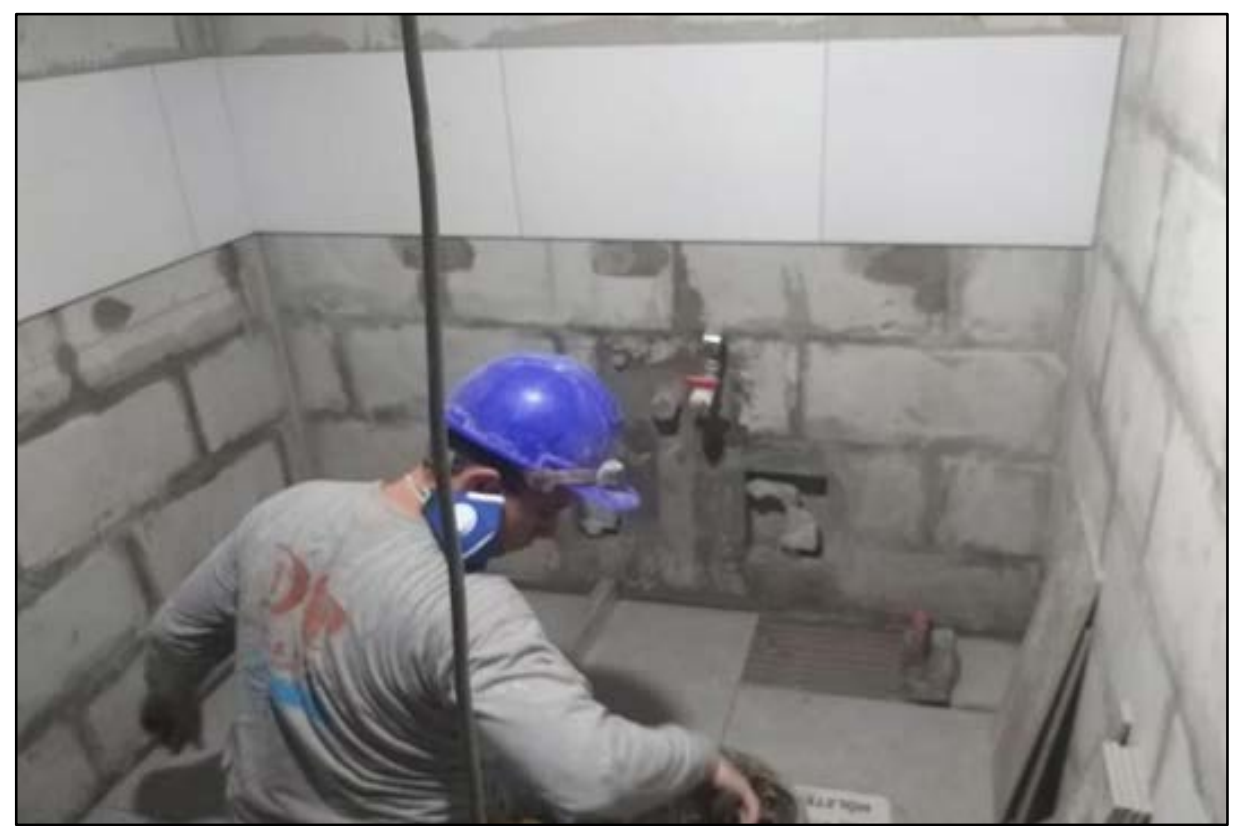

Fuente: Elaboración Propia 
b) Identificación y Clasificación de las Actividades:

Teniendo en cuenta el proceso constructivo mencionado, se identifica las actividades que componen el enchape del cerámico, como, por ejemplo: la colocación del enchape, la colocación de pegamento, el asentado del enchape, la nivelación del enchape, transporte de materiales/herramientas, preparación de la mezcla de pegamento, trazo para colocar los enchapes, medir y cortar los enchapes, limpieza en los ambientes, entre otras actividades.

Estas actividades se clasifican dentro de las categorías de trabajo productivo y contributorio. A continuación, en la Tabla $\mathrm{N}^{\circ} 7$ se muestra la clasificación de las actividades identificadas, en donde también se consideran los trabajos no contributorios generalmente involucrados en las actividades:

Tabla Nº 6: Clasificación de las actividades de Enchape de Cerámico

\begin{tabular}{|c|l|}
\hline \multicolumn{2}{|c|}{ CLASIFICACIÓN DEL TRABAJO } \\
\hline \multicolumn{2}{|c|}{ TRABAJO PRODUCTIVO (TP) } \\
\hline \multicolumn{2}{|c|}{ Colocación de Enchape } \\
\hline CP & Colocación de Pegamento \\
\hline AP & Asentado de Enchape \\
\hline NE & Nivelación de Enchape \\
\hline CC & Colocación de Crucetas \\
\hline \multicolumn{2}{|l|}{} \\
\hline \multicolumn{2}{|l|}{ TRABAJO CONTRIBUTORIO (TC) } \\
\hline TM & Transporte de Herramientas/Materiales \\
\hline BM & Preparación de Mezcla \\
\hline TE & Trazo de Enchape \\
\hline COE & Corte de Enchape \\
\hline I & Instrucciones \\
\hline L & Limpieza de área de trabajo \\
\hline & \\
\hline NF & Necesidades Fisiológicas \\
\hline MI & Movilización Improductiva \\
\hline TO & Tiempo Oscioso \\
\hline E & Trabajo Rehecho \\
\hline & Esperas \\
\hline
\end{tabular}

Fuente: Elaboración Propia. 
c) Descripción de la Cuadrilla

La cuadrilla está conformada por 2 personas (1 operario y 1 ayudante). Los operarios realizan el trabajo productivo (colocación del pegamento, colocación, asentado y nivelación del enchape), mientras que los ayudantes procuran preparar todo lo necesario para que los operarios ejecuten sus tareas sin inconvenientes, como traer los materiales a la zona de trabajo, preparar el pegamento, cortar el enchape y limpiar el área de trabajo.

\subsubsection{ASENTAdo DE LAdRILLO BLANCO}

a) Proceso Constructivo:

Definir el trazo topográfico, luego se procede a colocar el acero de refuerzo vertical, esto define la posición de la primera fila de ladrillos, teniendo cuenta la posición de los alveolos. Para continuar con las siguientes filas de ladrillo se hace mediante el mortero grueso de concreto, adicional a ello se coloca acero de refuerzo horizontal, cada tres filas de ladrillo blanco. Finalmente se controla el nivel y aplomado.

Los materiales que se emplean en esta actividad son las barras de acero corrugado de $6 \mathrm{~mm}$ - 3/8”, los ladrillos blancos P-10 y P-12, la arena gruesa, el cemento y el agua. Por otro lado, las herramientas que se utilizan son las reglas de aluminio para el regleado, las planchas de batir para preparar el mortero, las bateas para colocar los agregados y preparar el mortero, y la cortadora eléctrica para el ladrillo blanco.

A continuación, se presentan las siguientes imágenes sobre el proceso constructivo del asentado de ladrillo blanco: 
Fotografía No 5: Asentado de Ladrillo Blanco - Edificio Twenty.

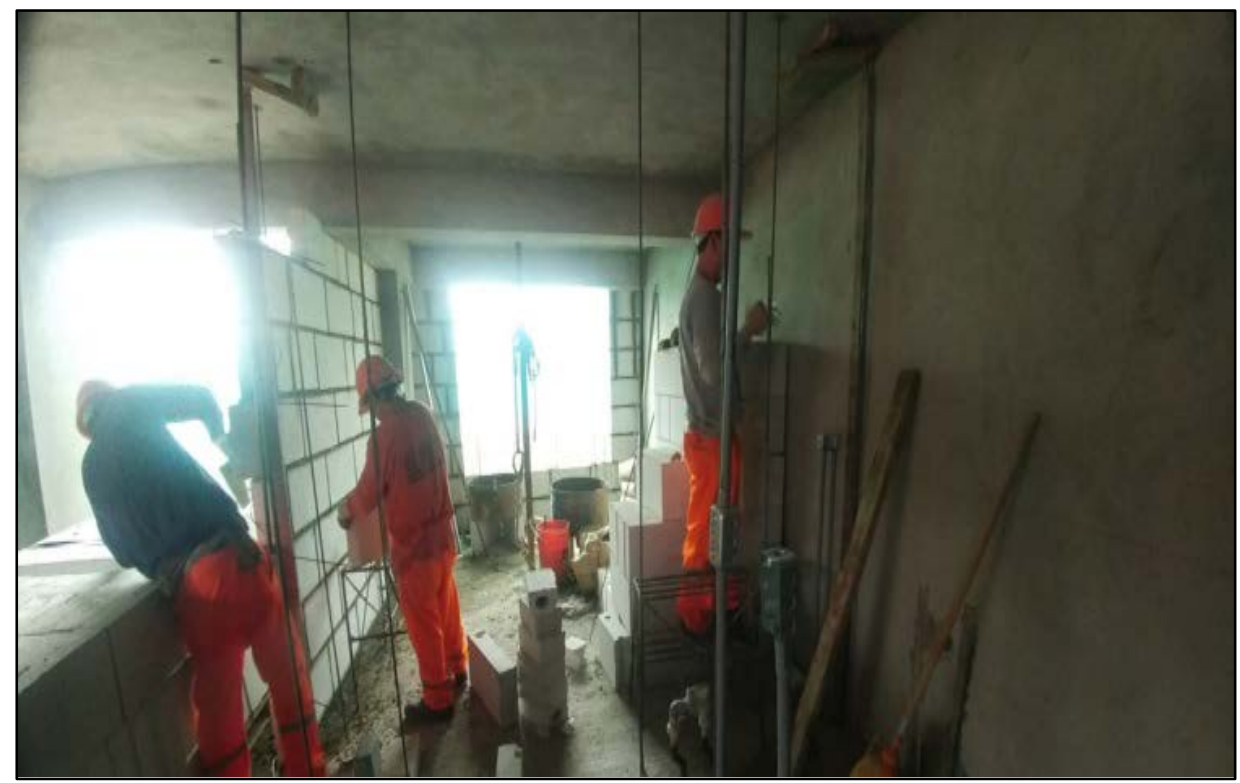

Fuente: Elaboración Propia.

Fotografía No 6: Asentado de Ladrillo Blanco - Edificio Twenty.

Fuente: Elaboración Propia. 
Fotografía No 7: Asentado de Ladrillo Blanco - Edificio Twenty.

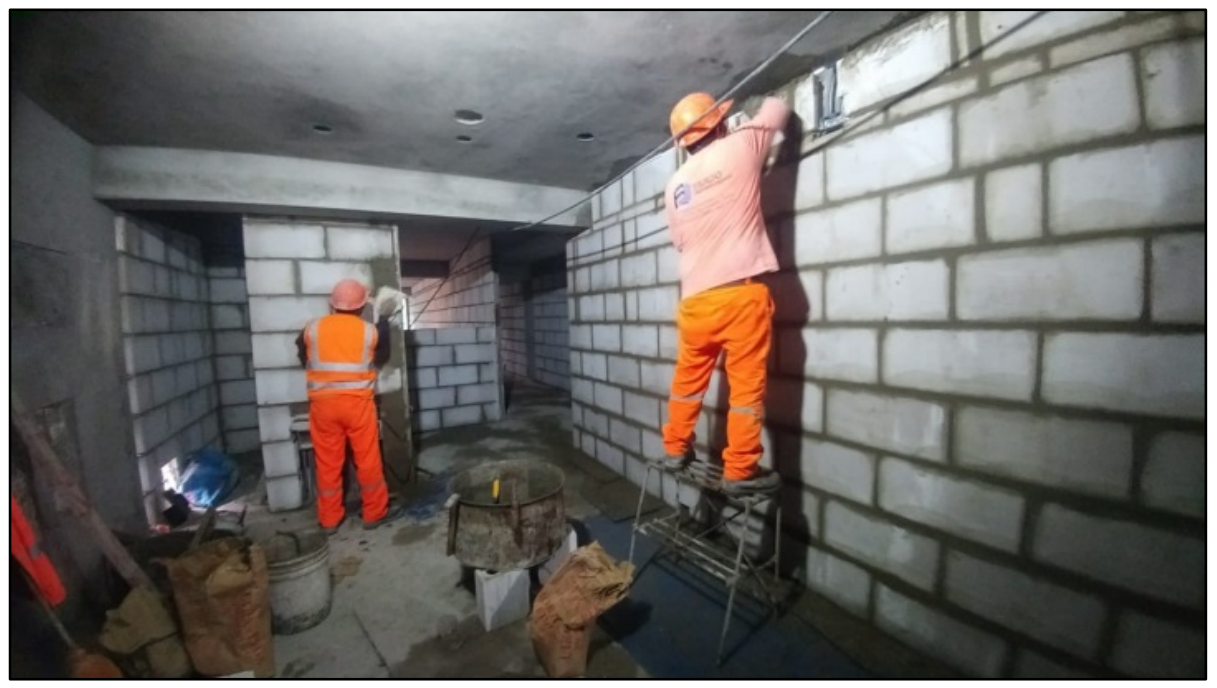

Fuente: Elaboración Propia.

d) Identificación y Clasificación de las Actividades:

Teniendo en cuenta el proceso constructivo mencionado, se identifica las actividades que componen el asentado de ladrillo blanco como, por ejemplo: asentado de ladrillo, preparación y colocación del mortero grueso, transporte de ladrillos y del mortero, colocación de varillas horizontales, medir y cortar ladrillos blancos, entre otros.

Estas actividades se clasifican dentro de las categorías de trabajo productivo y contributorio. A continuación, en la Tabla $N^{\circ} 7$ se muestra la clasificación de las actividades identificadas, en donde también se consideran los trabajos no contributorios generalmente involucrados en las actividades: 
Tabla Nº 7: Clasificación de las actividades de Asentado de Ladrillo Blanco.

\begin{tabular}{|c|c|}
\hline \multicolumn{2}{|r|}{ CLASIFICACIÓN DEL TRABAJO } \\
\hline \multicolumn{2}{|r|}{ TRABAJO PRODUCTIVO (TP) } \\
\hline $\mathrm{CM}$ & Colocación de Mortero Grueso \\
\hline $\mathrm{CA}$ & Colocación de Concreto en Alveólos \\
\hline $\mathrm{AL}$ & Asentado de Ladrillo Blanco \\
\hline NA & Nivelación y Aplomado \\
\hline \multicolumn{2}{|r|}{ TRABAJO CONTRIBUTORIO (TC) } \\
\hline $\mathrm{TH}$ & Transporte de Herramientas/Materiales \\
\hline TM & Transporte de Mezcla \\
\hline PM & Preparación de Mezcla \\
\hline $\mathrm{CH}$ & Colocación de Varillas Corrugadas Horizontales \\
\hline ML & Medir para cortar ladrillo \\
\hline $\mathrm{CL}$ & Corte de Ladrillo \\
\hline $\mathrm{L}$ & Limpieza de área de trabajo \\
\hline 1 & Instrucciones \\
\hline PV & Perforación para el anclaje de Varillas corrugadas \\
\hline $\mathrm{AV}$ & Aclaje de Varillas Corrugadas \\
\hline CR & Colocación de Reglas para Asentado \\
\hline $\mathrm{O}$ & Otros \\
\hline \multicolumn{2}{|r|}{ TRABAJO NO CONTRIBUTORIO (TNC) } \\
\hline $\mathrm{NF}$ & Necesidades Fisiológicas \\
\hline $\mathrm{MI}$ & Movilización Improductiva \\
\hline TO & Tiempo Oscioso \\
\hline TR & Trabajo Rehecho \\
\hline $\mathrm{E}$ & Esperas \\
\hline
\end{tabular}

Fuente: Elaboración Propia.

e) Descripción de la Cuadrilla

La cuadrilla está conformada por 4 personas (3 operarios y 1 ayudante). Los operarios realizan el trabajo productivo (asentado de ladrillo, preparación y colocación de Mortero Grueso, y nivelación y aplomado del muro), mientras que los ayudantes procuran preparar todo lo necesario para que los operarios ejecuten sus tareas sin inconvenientes como transportar los materiales y los ladrillos blancos, anclar las varillas de acero verticales, cortar los ladrillos, entre otros. 


\subsection{LEVANTAMIENTO DE INFORMACIÓN Y ANÁLISIS DE RESULTADOS}

Del 21 de agosto al 28 de agosto se procedió con la toma de medidas utilizando la Carta Balance. Se realizó 13 mediciones para el enchape de cerámico y 11 mediciones para el asentado de ladrillo blanco en diferentes horas del día. En la Tabla Nº8 se observa la actividad, la fecha y la hora en la que se tomaron las mediciones correspondientes.

Tabla No 8: Clasificación de las actividades de Asentado de Ladrillo Blanco.

\begin{tabular}{|c|l|c|c|}
\hline \multicolumn{4}{|c}{ CARTA BALANCE - EDIFICIO TWENTY } \\
\hline N $^{\circ}$ & \multicolumn{1}{|c|}{ ACTIVIDAD } & FECHA & HORA \\
\hline 1 & Asentado de Ladrillo & $21 / 08 / 2019$ & $08: 30: 00$ a.m. \\
\hline 2 & Asentado de Ladrillo & $21 / 08 / 2019$ & $10: 15: 00$ a.m. \\
\hline 3 & Asentado de Ladrillo & $21 / 08 / 2019$ & $01: 50: 00$ p.m. \\
\hline 4 & Asentado de Ladrillo & $21 / 08 / 2019$ & $03: 25: 00$ p.m. \\
\hline 5 & Asentado de Ladrillo & $22 / 08 / 2019$ & $08: 25: 00$ a.m. \\
\hline 6 & Asentado de Ladrillo & $22 / 08 / 2019$ & $10: 20: 00$ a.m. \\
\hline 7 & Asentado de Ladrillo & $22 / 08 / 2019$ & $01: 27: 00$ p.m. \\
\hline 8 & Asentado de Ladrillo & $22 / 08 / 2019$ & $03: 00: 00$ p.m. \\
\hline 9 & Enchape de Cerámico & $23 / 08 / 2019$ & $08: 30: 00$ a.m. \\
\hline 10 & Enchape de Cerámico & $23 / 08 / 2019$ & $10: 15: 00$ a.m. \\
\hline 11 & Enchape de Cerámico & $23 / 08 / 2019$ & $01: 12: 00$ p.m. \\
\hline 12 & Enchape de Cerámico & $23 / 08 / 2019$ & $02: 43: 00$ p.m. \\
\hline 13 & Enchape de Cerámico & $26 / 08 / 2019$ & $08: 20: 00$ a.m. \\
\hline 14 & Enchape de Cerámico & $26 / 08 / 2019$ & $10: 00: 00$ a.m. \\
\hline 15 & Enchape de Cerámico & $26 / 08 / 2019$ & $01: 13: 00$ p.m. \\
\hline 16 & Enchape de Cerámico & $26 / 08 / 2019$ & $03: 00: 00$ p.m. \\
\hline 17 & Enchape de Cerámico & $27 / 08 / 2019$ & $08: 37: 00$ a.m. \\
\hline 18 & Enchape de Cerámico & $27 / 08 / 2019$ & $10: 10: 00$ a.m. \\
\hline 19 & Enchape de Cerámico & $27 / 08 / 2019$ & $01: 10: 00$ p.m. \\
\hline 20 & Enchape de Cerámico & $27 / 08 / 2019$ & $02: 50: 00$ p.m. \\
\hline 21 & Enchape de Cerámico & $28 / 08 / 2019$ & $08: 34: 00$ a.m. \\
\hline 22 & Asentado de Ladrillo & $28 / 08 / 2019$ & $10: 18: 00$ a.m. \\
\hline 23 & Asentado de Ladrillo & $28 / 08 / 2019$ & $01: 19: 00$ p.m. \\
\hline 24 & Asentado de Ladrillo & $28 / 08 / 2019$ & $02: 55: 00$ p.m. \\
\hline
\end{tabular}

Fuente: Elaboración Propia.

A continuación, se presentan el promedio de los resultados obtenidos de las mediciones realizadas entre el 21 de agosto y el 28 de agosto para la actividad de Asentado de Ladrillo. Los siguientes gráficos muestran el porcentaje de la distribución de trabajo en 
la actividad en mención y el porcentaje de las actividades correspondientes a los trabajos productivos, contributorios y no contributorios.

Figura No 18: Distribución de Trabajo en Asentado de Ladrillo.

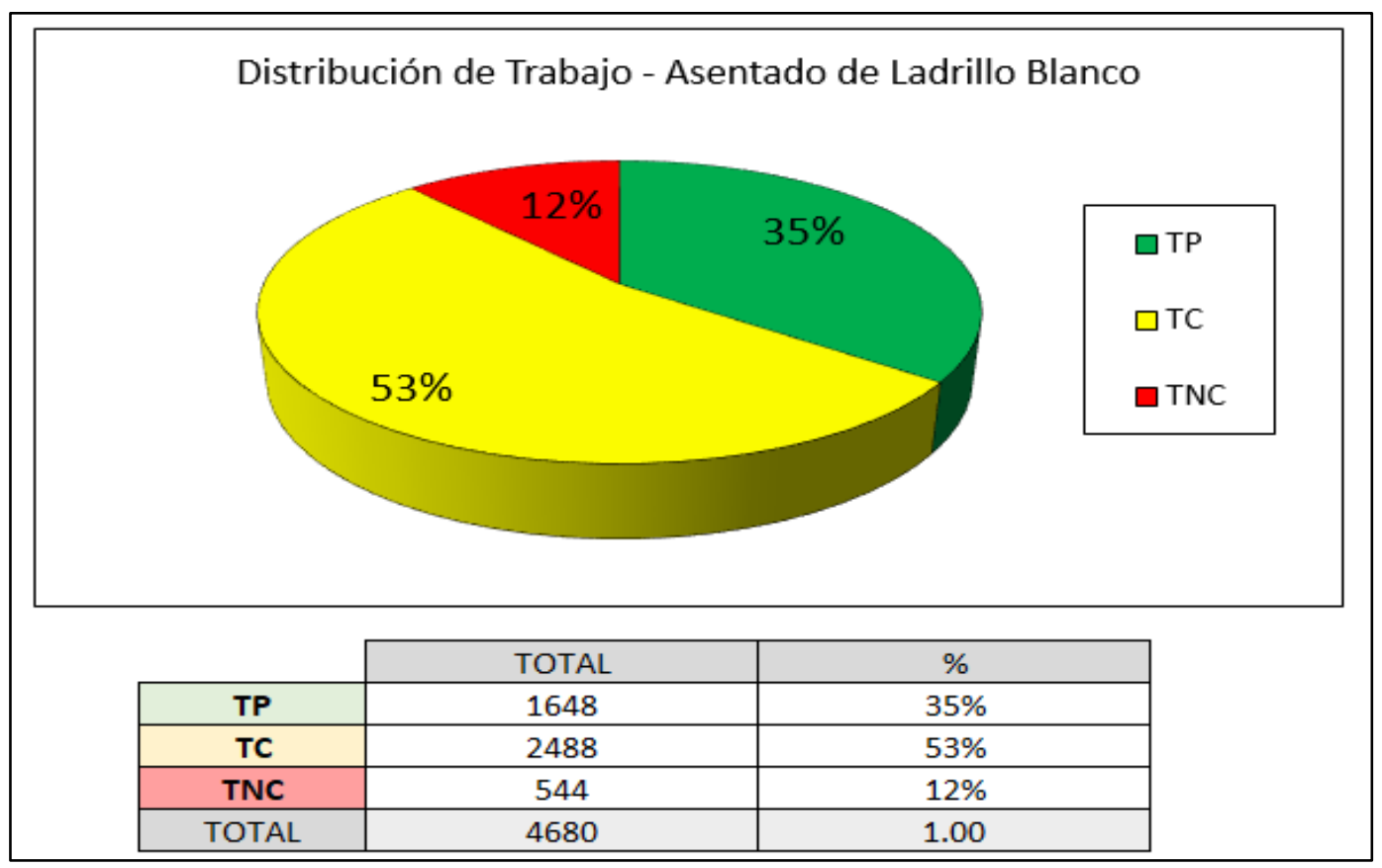

Fuente: Elaboración Propia.

Figura Nº 19: Distribución de Trabajo Productivo en Asentado de Ladrillo.

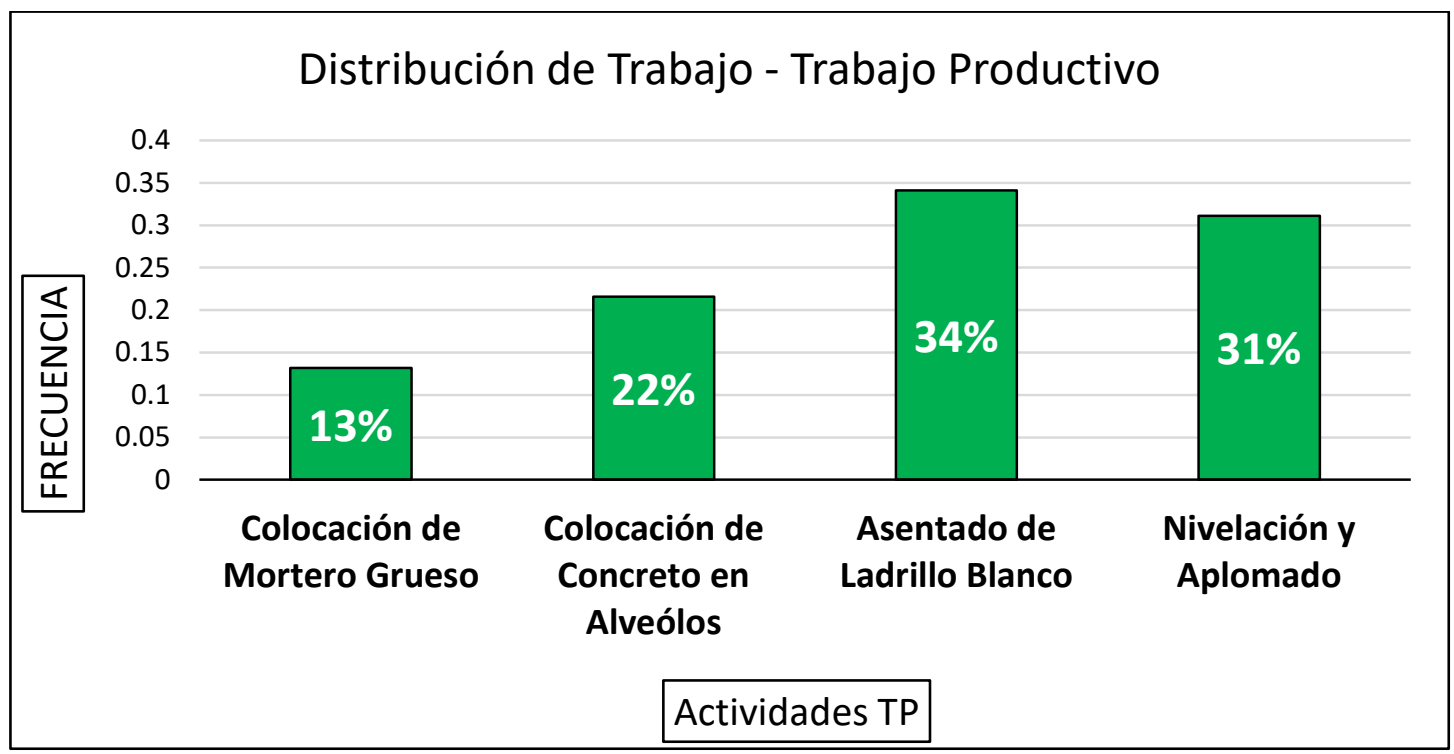

Fuente: Elaboración Propia. 
Figura No 20: Distribución de Trabajo Contributorio en Asentado de Ladrillo

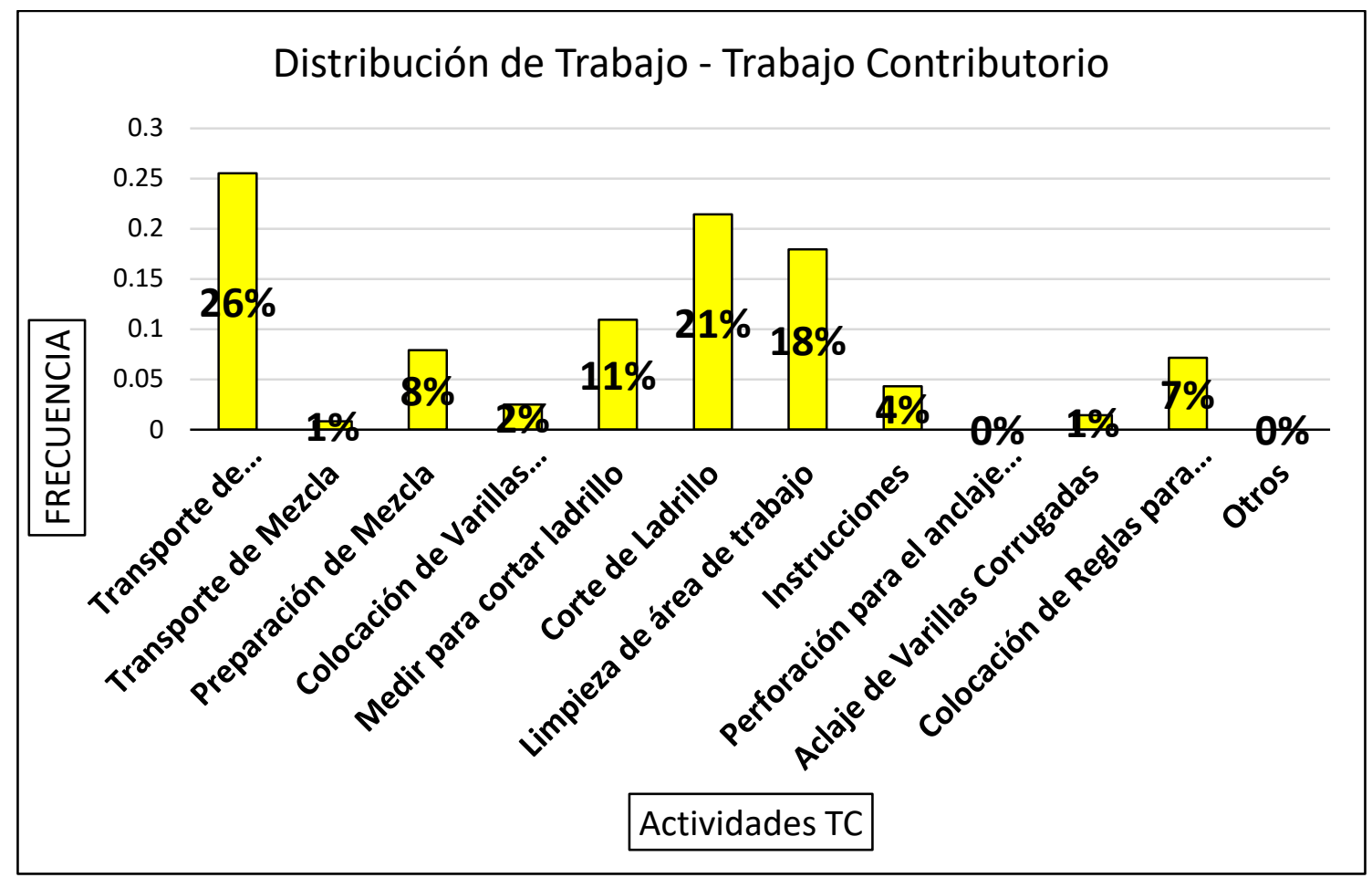

Fuente: Elaboración Propia.

Figura No 21: Distribución de Trabajo No Contributorio en Asentado de Ladrillo

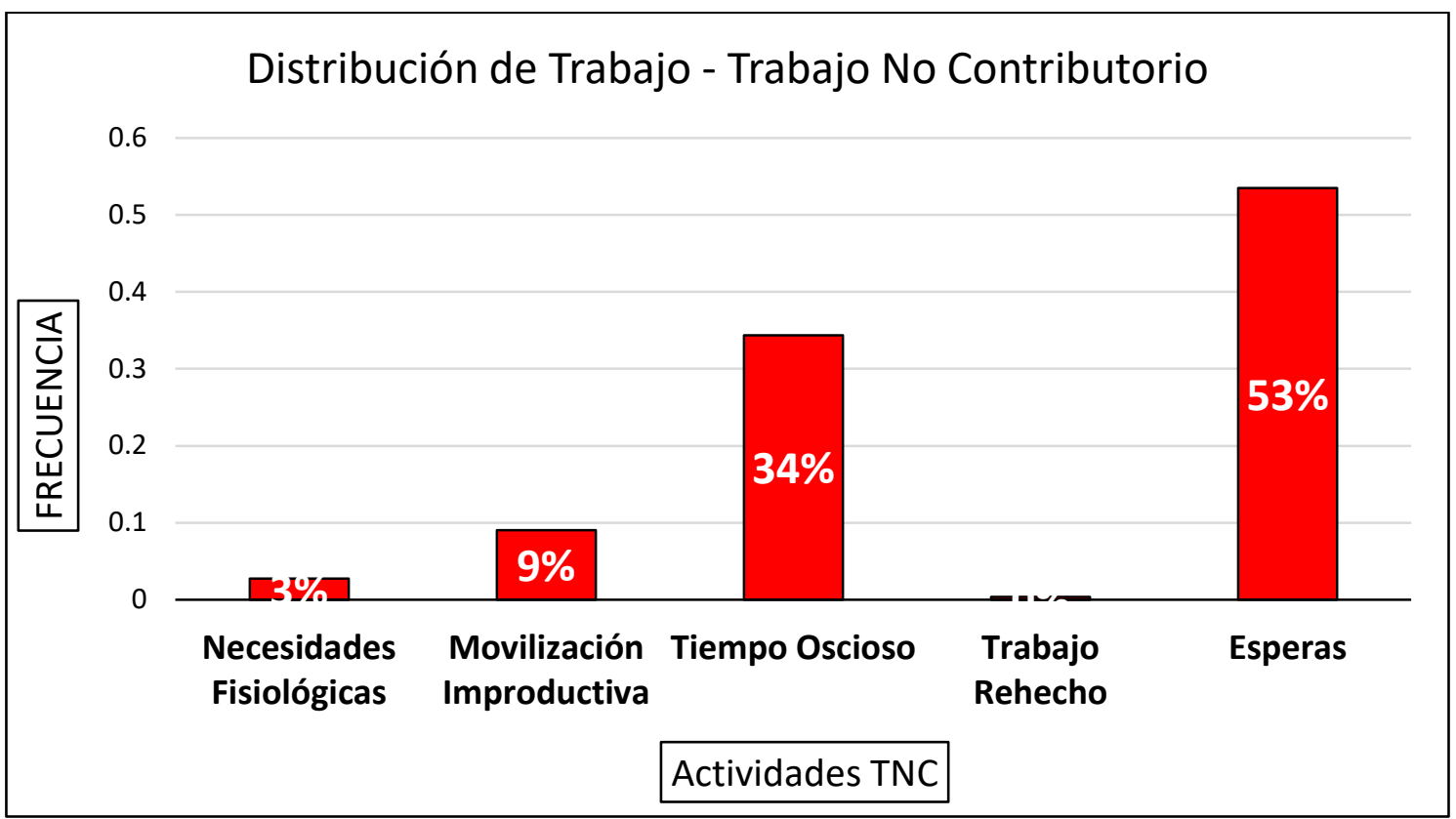

Fuente: Elaboración Propia. 
Como se puede observar en la Figura $\mathrm{N}^{\circ} 18$, la clasificación de trabajo que tiene mayor incidencia en la partida de asentado de ladrillo blanco es el trabajo contributorio con un 53\%. En la Figura $N^{\circ} 20$, se observa que las actividades más concurrentes dentro del trabajo contributorio son los siguientes:

- $\quad$ Transporte de Materiales/Herramientas: 26\%

- Corte de Ladrillo: 21\%

- $\quad$ Limpieza de Área de Trabajo: 18\%

Uno de los principales problemas que se observó durante las mediciones diarias en relación a esta actividad, es que los operarios emplearon una gran parte de su tiempo en las actividades señaladas, las cuales deberían ser ejecutadas por los ayudantes. Asimismo, el transporte de materiales/herramientas se ejecuta constantemente en todo el día, cuando se puede acarrear todos los materiales necesarios por la mañana para que en la tarde realizar gran parte del trabajo productivo. También, el tiempo de corte de ladrillo es muy alto e involucra tanto a los ayudantes como a los operarios en esta operación. Es por esta razón, que existe un alto porcentaje de trabajo contributorio en esta partida.

Por otro lado, en la Figura $\mathrm{N}^{\circ} 21$, las causas más frecuentes del trabajo no contributorio son el Tiempo Ocioso (34\%) y las Esperas (53\%). Como se muestra en las mediciones diarias, el Tiempo Ocioso existe principalmente en los operarios, por lo que existe una posibilidad de transformar este porcentaje de trabajo no contributorio a trabajo productivo.

A continuación, se presentan el promedio de los resultados obtenidos de las mediciones realizadas entre el 21 de agosto y el 28 de agosto para la actividad de Asentado de Ladrillo. Los siguientes gráficos muestran el porcentaje de la distribución de trabajo en la actividad en mención y el porcentaje de las actividades correspondientes a los trabajos productivos, contributorios y no contributorios. 
Figura No 22: Distribución de Trabajo en Enchape de Cerámico

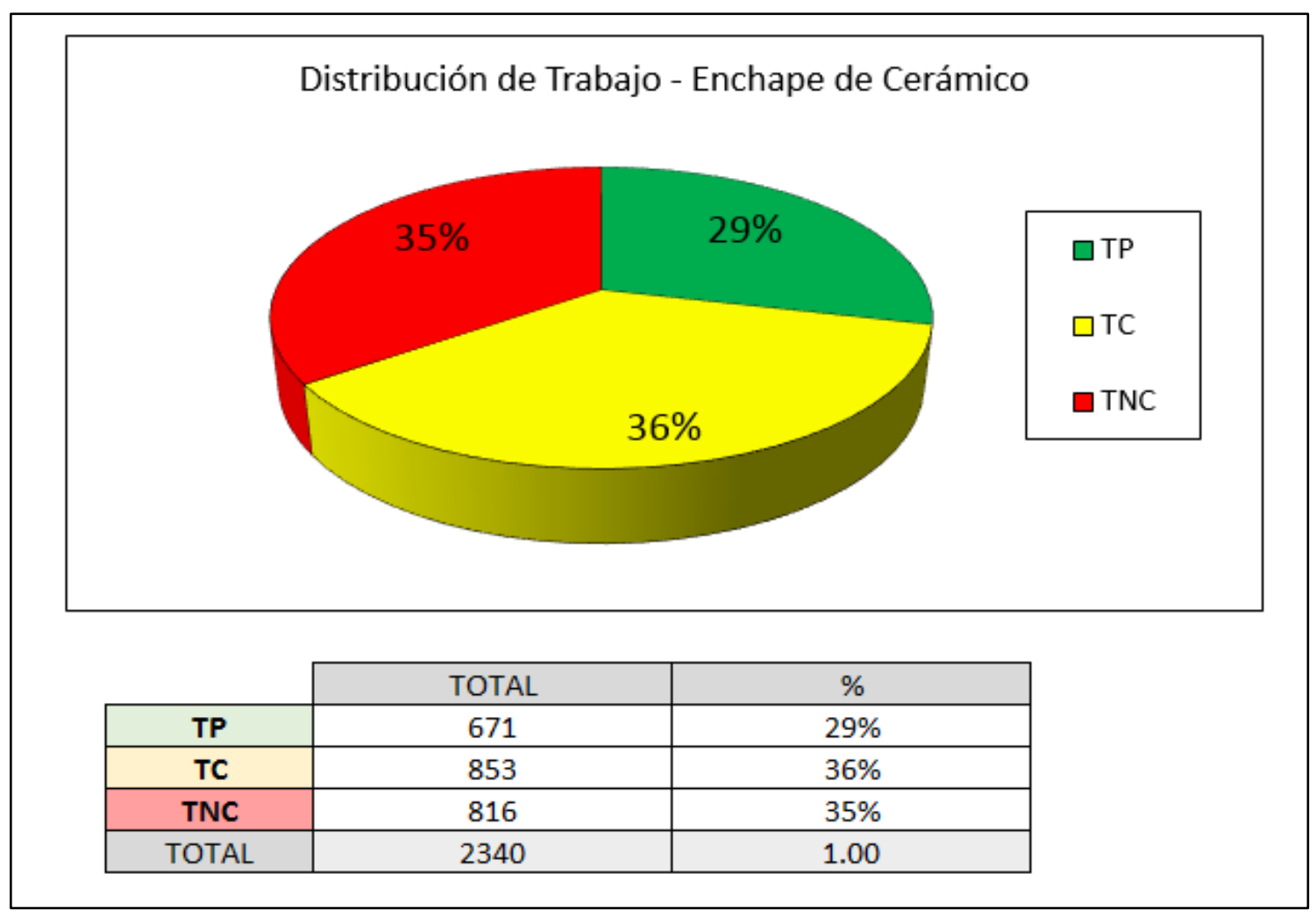

Fuente: Elaboración Propia.

Figura Nº 23: Distribución de Trabajo Productivo en Enchape de Cerámico

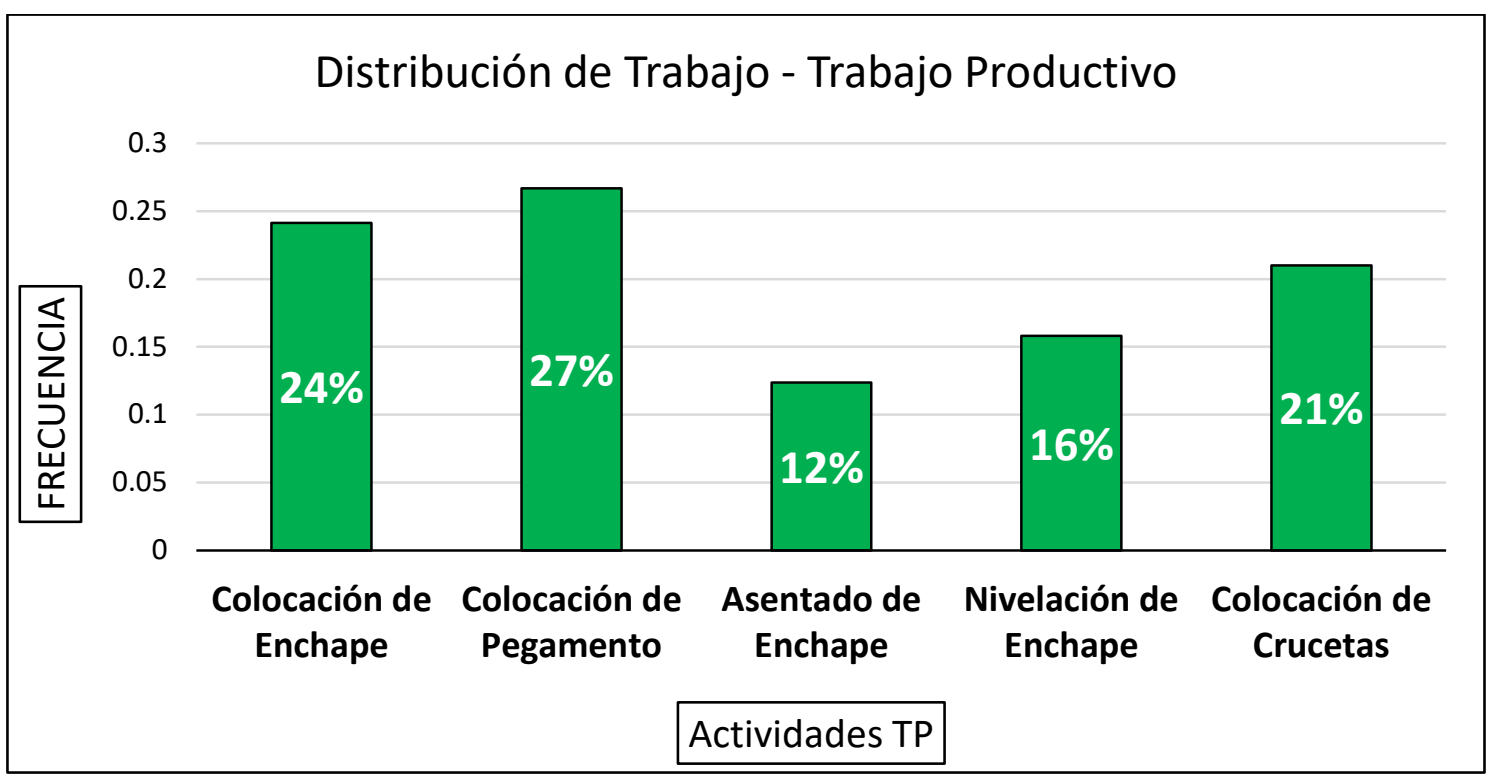

Fuente: Elaboración Propia. 
Figura Nº 24: Distribución de Trabajo Contributorio en Enchape de Cerámico

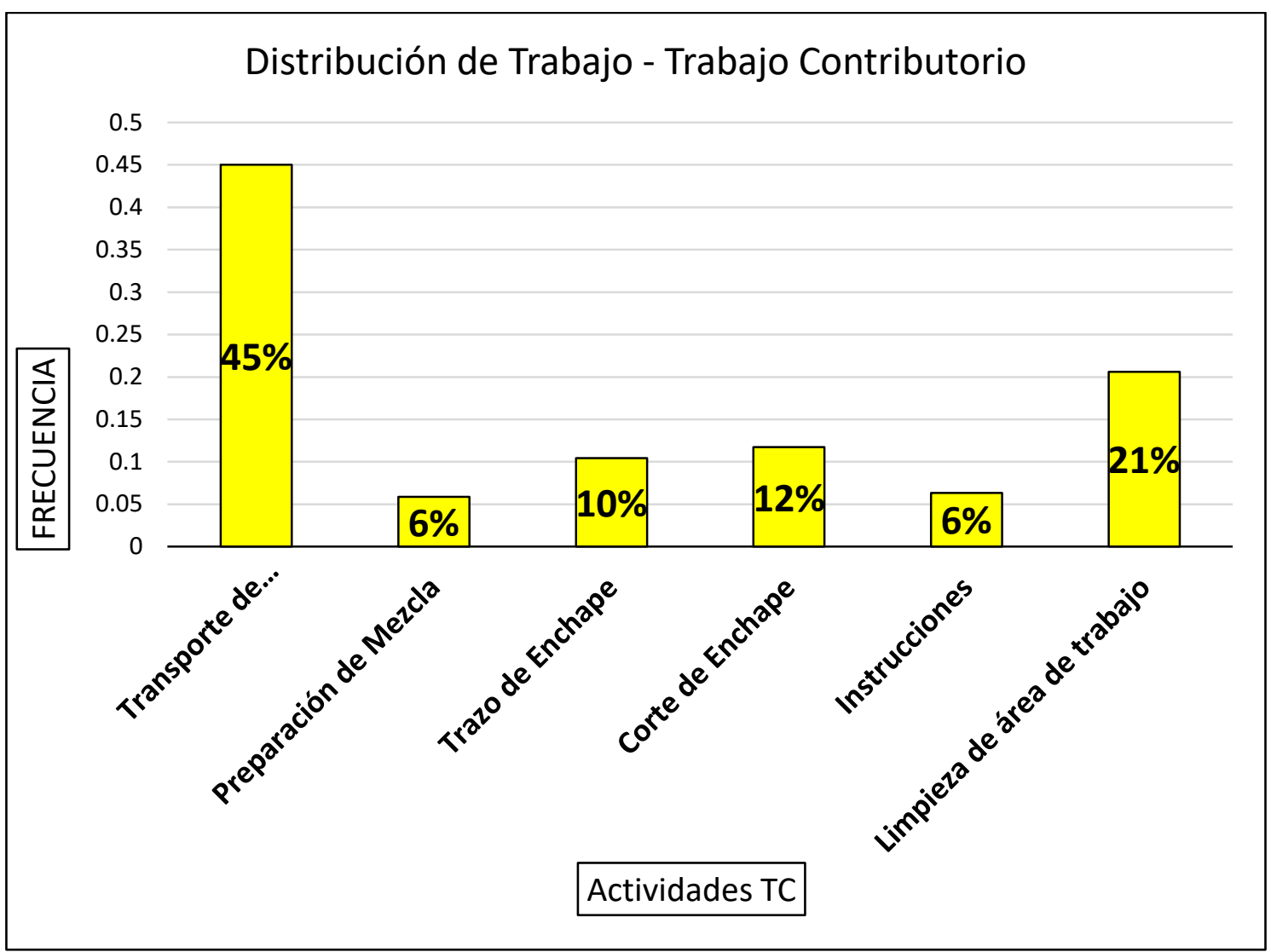

Fuente: Elaboración Propia.

Figura No 25: Distribución de Trabajo No Contributorio en Enchape de Cerámico

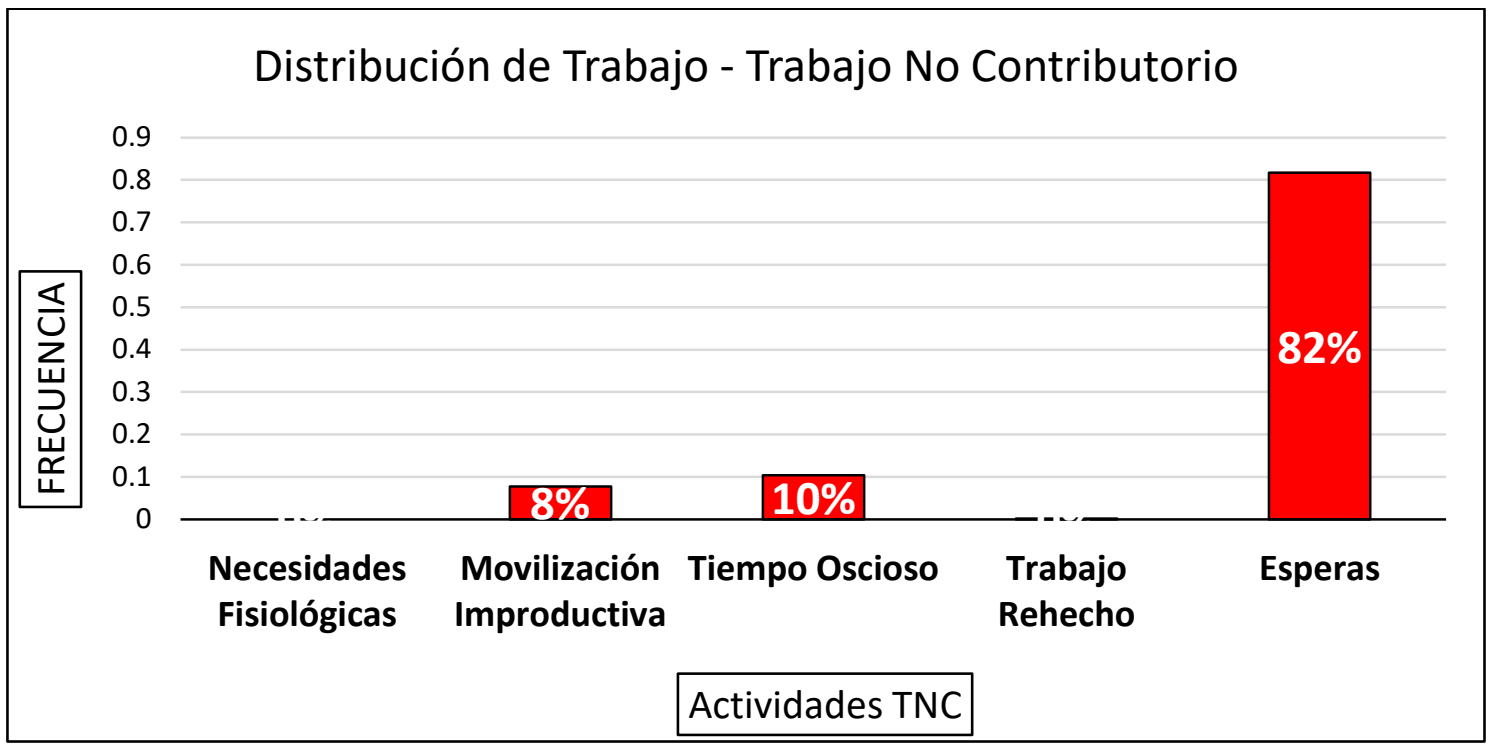

Fuente: Elaboración Propia. 
Como se puede observar en la Figura $\mathrm{N}^{\circ} 22$, el porcentaje de la clasificación de trabajo en la partida de Enchape de Cerámico es repartida entre las tres categorías. Cabe resaltar que existe un $35 \%$ de trabajo no contributorio, el cual se debe principalmente a las Esperas (82\%) como se muestra en la Figura $\mathrm{N}^{\circ} 25$.

Uno de los principales problemas que se observó durante las mediciones diarias en relación a esta actividad, es la falta de apoyo del ayudante al operario en los trabajos de habilitación o de acarreo de materiales. Asimismo, existieron esperas para la ejecución del enchapado del cerámico en ciertos ambientes puesto que faltaba ejecutar otros trabajos, como la colocación de cajas de pase antes de enchapar. No existía coordinación con los demás contratistas, puesto que hubo un día en donde ocurrió una controversia entre un albañil y el enchapador puesto que estaban solaqueando la cocina y el enchapador no podía proceder con su trabajo hasta que el albañil culminará su actividad.

Asimismo, se observó que el operario y el ayudante para el enchape del cerámico no tenían conocimiento de la cantidad de metros cuadrados que tenían que avanzar por día, así como que ambientes son prioridad en esta actividad. Se percibía una falta de seguimiento en esta partida.

Por otro lado, en la Figura $\mathrm{N}^{\circ} 24$, la causa más frecuente del trabajo contributorio es el transporte de materiales/herramientas (45\%), actividad que lo ejecutaba en gran parte el operario. 


\section{CAPÍtUlo IV: Propuesta DE MejORA y ANÁlisis DE}

\section{LOS RESULTADOS}

\subsection{MODELO PROPUESTO DE MEJORA DE LA SITUACIÓN}

Con los resultados de las Cartas Balance vistos en el capítulo anterior, se procedió con el análisis correspondiente para identificar los principales problemas que impiden el aumento de productividad durante el día en las partidas de Asentado de Ladrillo Blanco y Enchape de Cerámico.

Una vez identificado los problemas, se formularon las propuestas de solución con el objetivo de aumentar el trabajo productivo en las partidas en mención. Como resultado, en la Tabla N³1 se muestra los problemas identificados y la solución propuesta, por parte de los autores en la partida de Asentado de Ladrillo Blanco. Además de ello se muestra el tipo de trabajo productivo, contributorio, no contributorio, que será afectado directamente y cuál será su posible conversión.

Tabla № 9: Identificación de Problemas y Propuesta de Solución - Asentado de Ladrillo Blanco

\begin{tabular}{|c|c|c|c|}
\hline \multicolumn{4}{|l|}{ PARTIDA: ASENTADO DE LADRILLOO BLANCO } \\
\hline \multirow{2}{*}{ PROBLEMA IDENTIFICADO } & \multirow{2}{*}{ PROPUESTA DE SOLUCIÓN } & \multicolumn{2}{|c|}{ EFECTO } \\
\hline & & REDUCE & APORTA \\
\hline Instalaciones Sanitarias y Eléctricas no liberadas al $100 \%$ & $\begin{array}{l}\text { Implementar reuniones con la metodología Last Planner } \\
\text { para identificación y liberación de restricciones con las } \\
\text { cuadrillas }\end{array}$ & TNC & TP, TC \\
\hline \multirow{2}{*}{$\begin{array}{l}\text { Transporte de Materiales/Herramientas es la actividad que toma mas } \\
\text { tiempo del trabajo contributorio. }\end{array}$} & $\begin{array}{l}\text { Reorganización de las actividades de acarreo de materiales, } \\
\text { para que se realice por las mañanas, }\end{array}$ & TC & TP \\
\hline & $\begin{array}{l}\text { Los operarios no deben realizar actividades de acarreo de } \\
\text { materiales, solo estará desginado a los oficiale o ayudantes }\end{array}$ & TC, TNC & TP \\
\hline $\begin{array}{l}\text { Se hace notar que no existe un orden adecuado para establecer las } \\
\text { cuadrillas cuando se trabaja en niveles distintos }\end{array}$ & Restructuración de cuadrillas para el trabajo en niveles distintos & TNC & TC, TP \\
\hline La Limpieza, en esta actividad, excede 1.5 horas al día & Exigir orden y limpieza en la áreas de trabajo de las cuadrillas & TC & TP \\
\hline \multirow{2}{*}{$\begin{array}{l}\text { El corte y habilitación de ladrillos es permanente en el día y esta } \\
\text { condicionado que exista alguna restricción, por consiguiente se tiene } \\
\text { mucho tiempo de espera del personal designado al corte. }\end{array}$} & $\begin{array}{l}\text { Modulación de muros en campo para evitar esperas en el } \\
\text { personal } \\
\text { asignado a corte. }\end{array}$ & TC & TP \\
\hline & $\begin{array}{l}\text { Realizar un layout para afinar la distribución de las cuadrillas y } \\
\text { la maquina de corte para aminorar el transporte de materiales. }\end{array}$ & TNC, TC & TP \\
\hline $\begin{array}{l}\text { Alta generación de polvo en la maquina de cortar ladrillo provoca que el } \\
\text { rendimiento del personal baje, debido a alergias. }\end{array}$ & $\begin{array}{l}\text { Encapsulamiento y mejor ventilación en la ubicación de la } \\
\text { maquina de corte de ladrillo }\end{array}$ & TNC & TC \\
\hline Alto porcentaje de Tiempo Oscioso en el trabajo no contributorio & Motivación al personal con reconocimiento e incentivos & TNC & TP \\
\hline
\end{tabular}

Fuente: Elaboración Propia.

Por otro lado, en la partida de Enchape de Cerámico se observan problemas diferentes a los identificados en la partida de Asentado de Ladrillo Blanco. Los problemas identificados en esta partida corresponden principalmente con la coordinación entre 
subcontratistas y con la misma subcontratista de enchape, la cual no tiene en claro el horizonte de trabajo que debe cumplir para alcanzar los objetivos propuestos por el ingeniero de campo. Esto se traduce en un aproximado de $40 \%$ de trabajo no contributorios. A continuación, en la Figura №26 se observa los problemas identificados que afectan directamente a la productividad de esta partida y la propuesta de solución para estos.

Figura Nº 26: Identificación de Problemas y Propuesta de Solución - Enchape de Cerámico

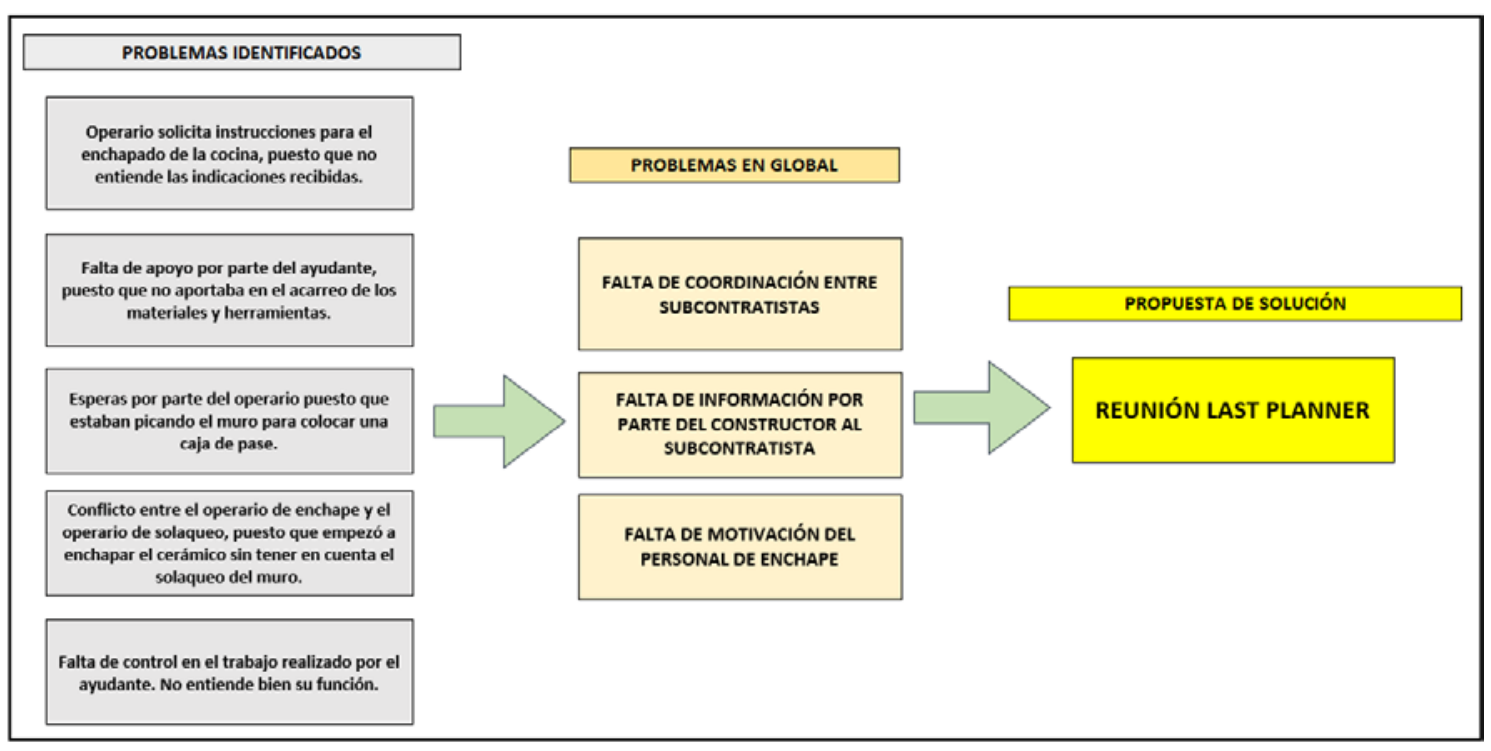

Fuente: Elaboración Propia

\subsection{Planes DE ACCIÓN PARA LA IMPLEMENTACión DE LA PROPUESTA}

Los planes de mejoramiento fueron implementados gradualmente de lo más sencillo hasta el más complicado. Sin embargo, en las dos partidas analizadas el mayor problema identificado fue la falta de coordinación entre las partes involucradas en la ejecución de los trabajos, ya que las restricciones crecían a medida que se ejecutaban más partidas en la misma área de trabajo y esto se reflejaba que existiera mayor tiempo no contributorio (TNC) como efecto de ello un aumento sustancial de las esperas en las cuadrillas de trabajo. 
Por lo tanto, se inició con la implementación del Sistema Last Planner, lo cual sumado a las otras soluciones propuestas dieron efecto positivo para alcanzar los objetivos de este trabajo de investigación.

Es así como se inició las reuniones de coordinación con todos los involucrados, donde se fijaba los objetivos y las fechas de cumplimiento para cada cuadrilla de trabajo.

Fotografía No 8: Reuniones Last Planner - Edificio Twenty.

Fuente: Elaboración Propia.

Fotografía N 9: Liberación de restricciones con los jefes de grupo - Edificio Twenty.

Fuente: Elaboración Propia. 
El siguiente paso fue la planificación detallada a corto plazo conocida como planificación semanal, en donde se puede distinguir y/o discriminar los conflictos que pudiesen existir a la hora de la ejecución, con ello reducir de manera considerable los trabajos no contributorios específicamente las esperas, que son las más frecuentes en las partidas analizadas.

Fotografía N 10: Planificación semanal y liberación de restricciones - Edificio Twenty.

Fuente: Elaboración Propia.

Fotografía No 11: Resultado de planificación semanal - Edificio Twenty.

Fuente: Elaboración Propia.

Adicionalmente, se realizó el análisis del flujo de trabajo para ambas partidas que se analiza en este trabajo de investigación ver figuras $\mathrm{N}^{\circ} 27$ y $\mathrm{N}^{\circ} 28$. Esto ayudó a optimizar 
la distribución de las cuadrillas y con ello la eliminación de tiempos improductivos como esperas por falta de espacio, tiempo de transporte de materiales/herramientas, etc.

Figura $\mathrm{N}^{\mathrm{o}}$ 27: Flujo de trabajo Asentado de Ladrillo Blanco

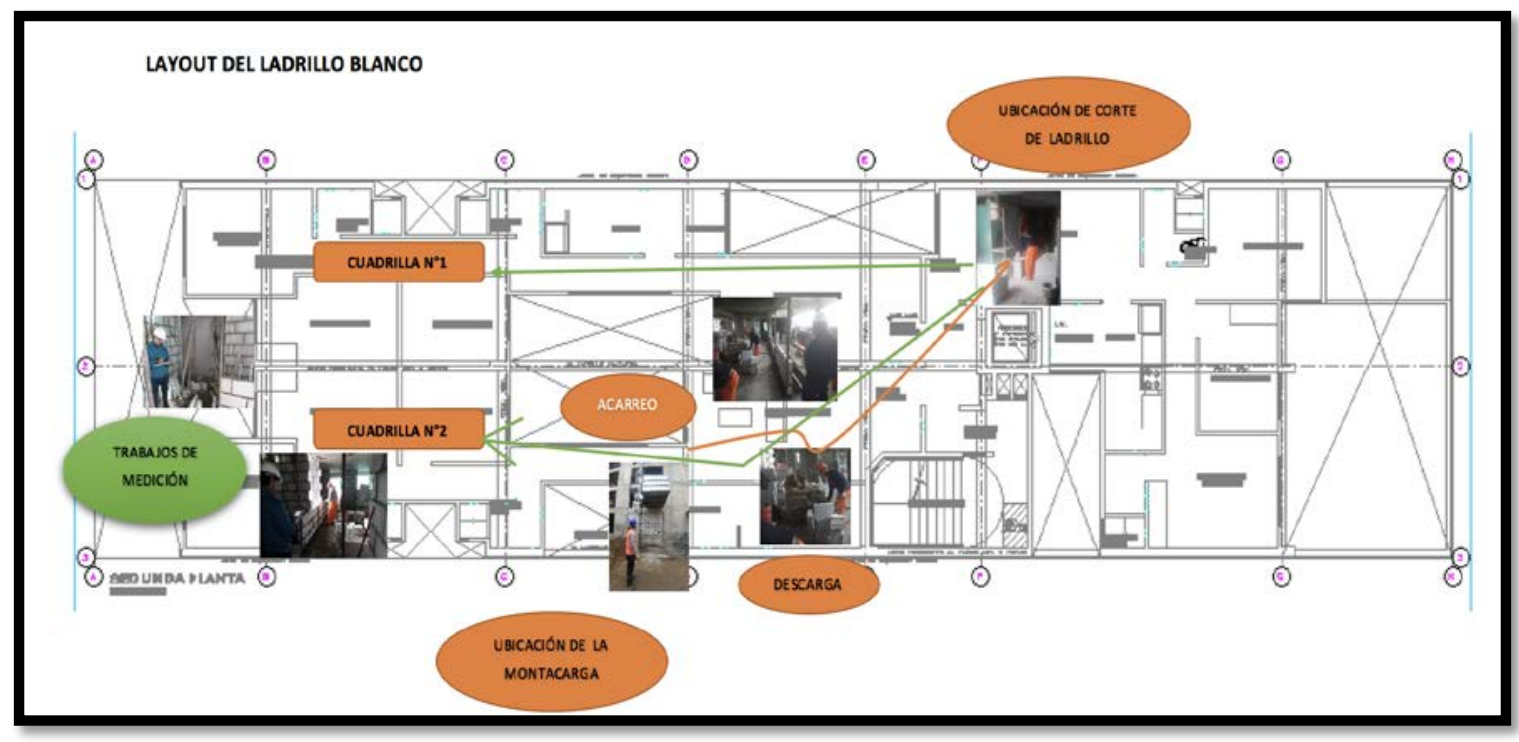

Fuente: Elaboración Propia.

Figura Nº 28: Flujo de trabajo Enchape de Cerámico

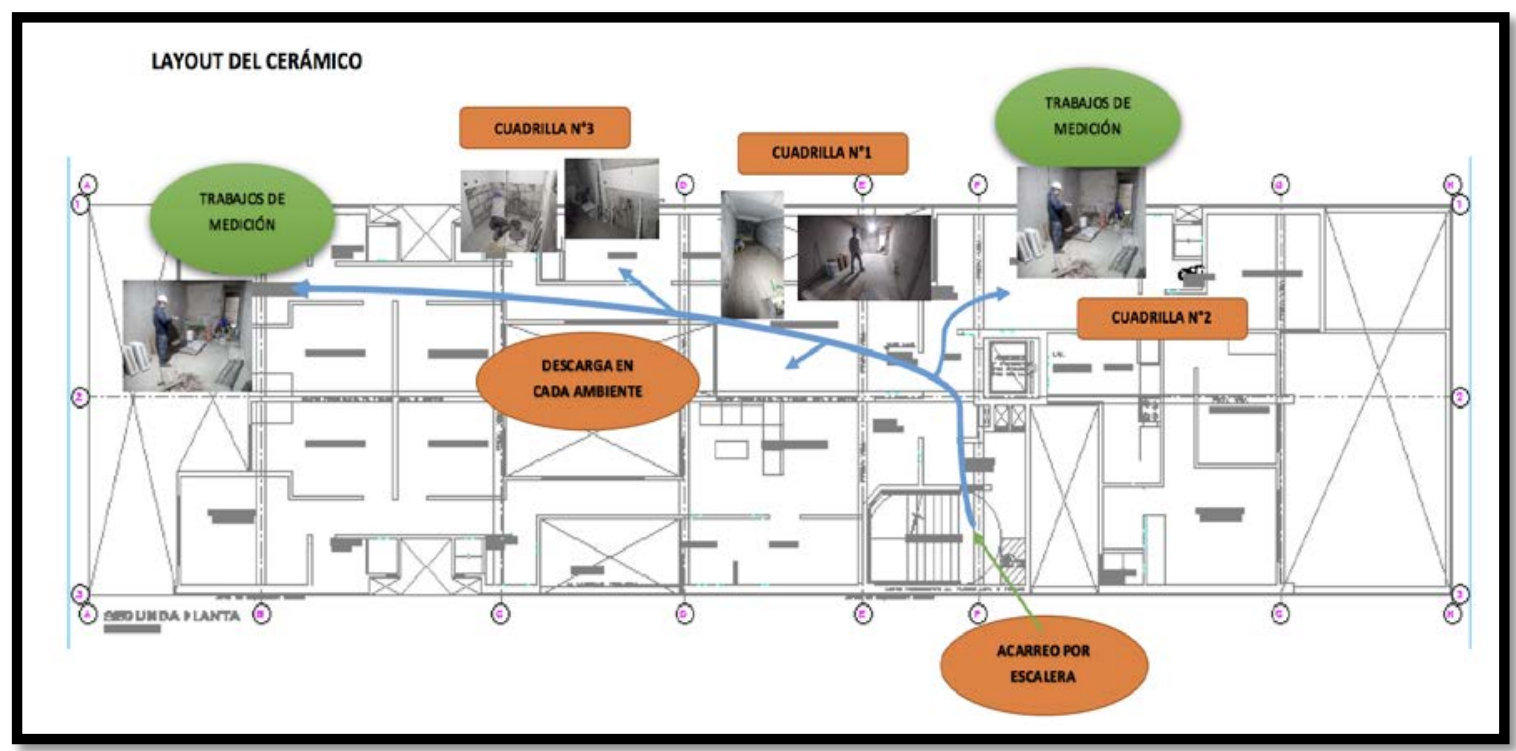

Fuente: Elaboración Propia. 


\subsection{RESULTADOS DESPUÉS DE LA IMPLEMENTACIÓN DE LA MEJORA}

Una vez hecha la implementación de nuestra mejora se procedió a realizar las medidas correspondientes para observar la magnitud de los efectos positivos o negativos de los cambios propuestos. A continuación, se muestra los resultados de la medición en las cuadrillas de Asentado de ladrillo y Enchape de cerámico respectivamente:

Figura No 29: Distribución Promedio de Trabajo de Asentado de Ladrillo - Post Implementación de Mejoras

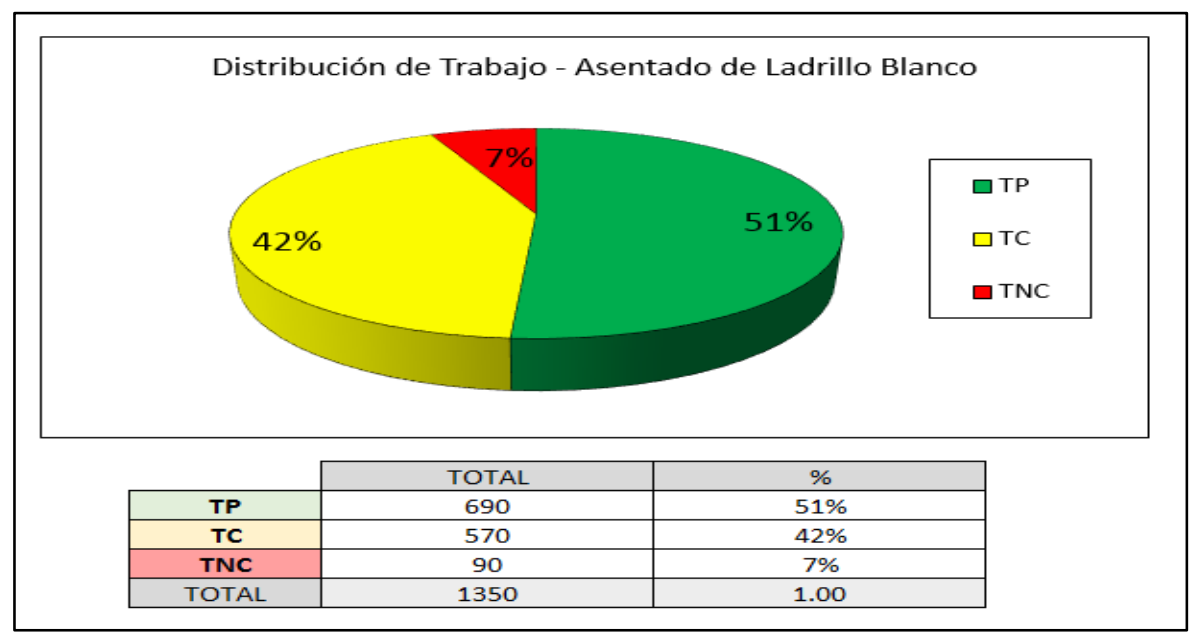

Fuente: Elaboración Propia.

Figura No 30: Distribución Promedio de Trabajo Productivo de Asentado de Ladrillo -

Post Implementación de Mejoras

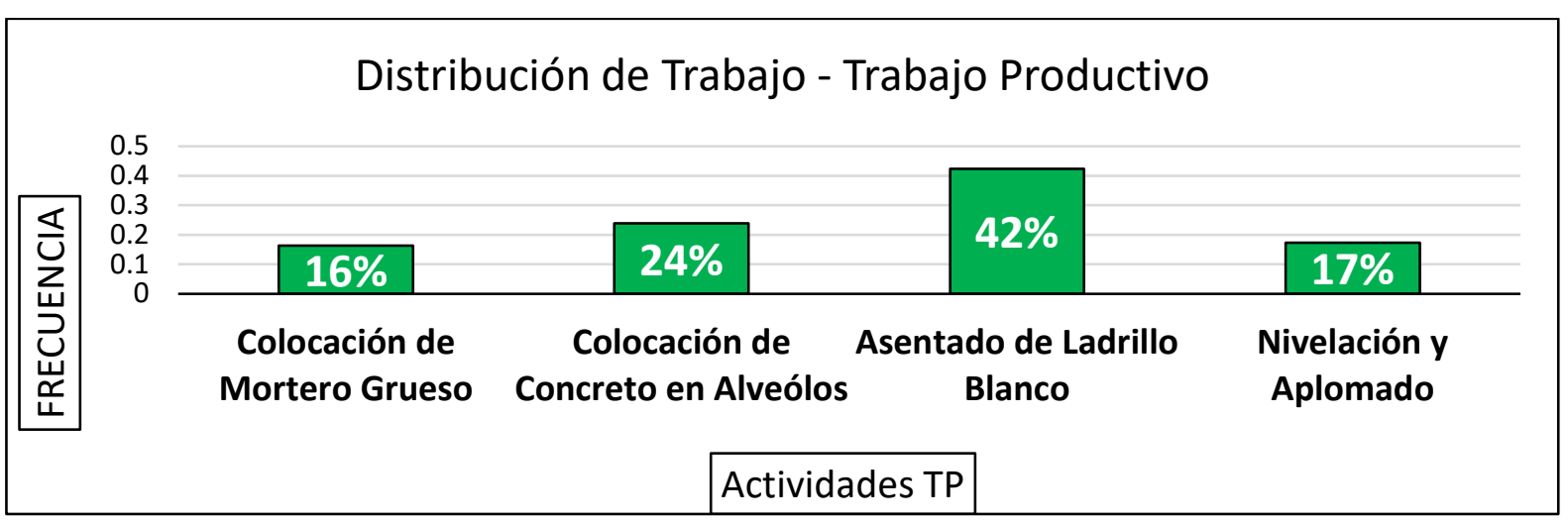

Fuente: Elaboración Propia. 
Figura No 31: Distribución Promedio de Trabajo Contributorio de Asentado de Ladrillo - Post Implementación de Mejoras

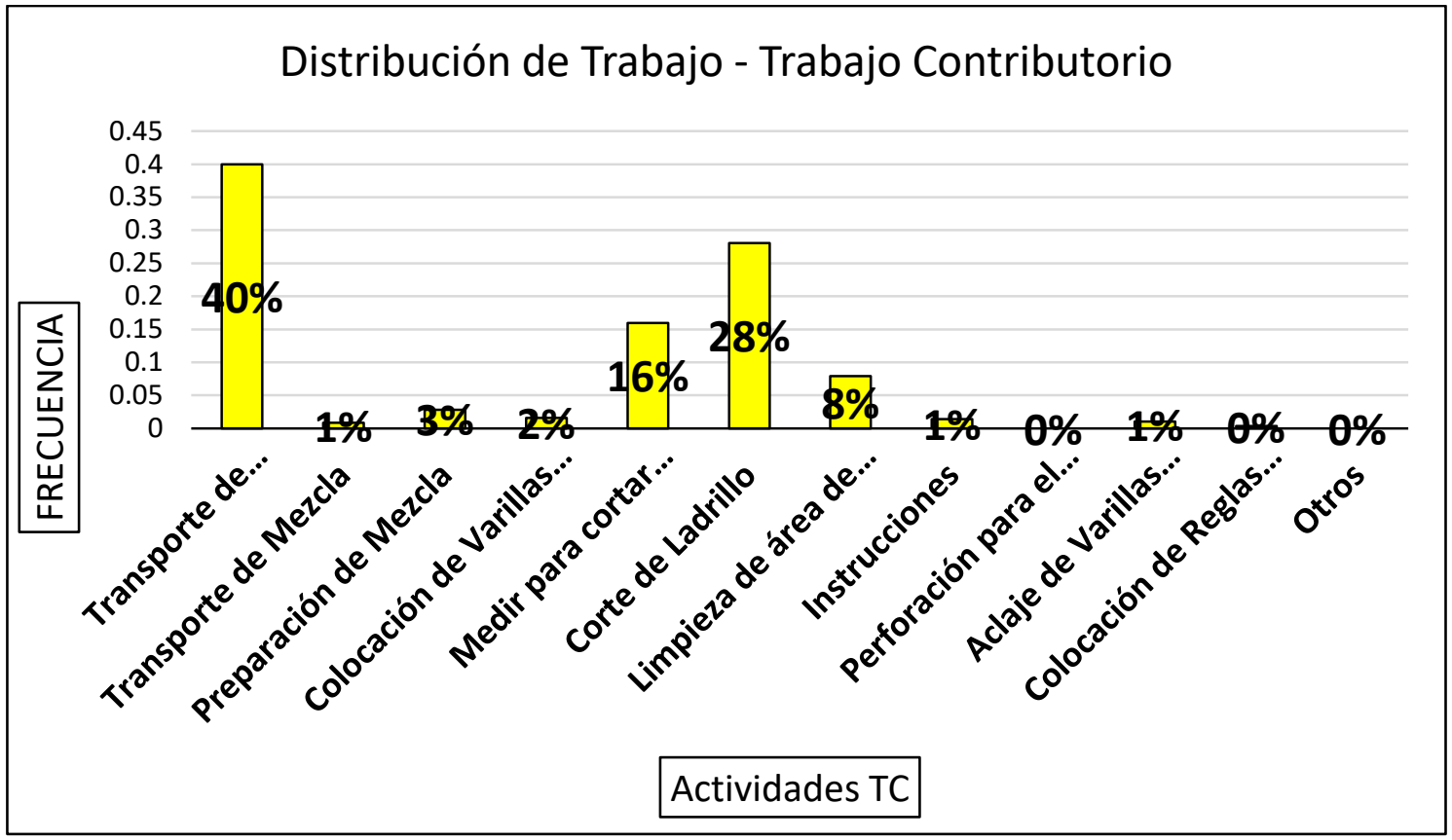

Fuente: Elaboración Propia.

Figura No 32: Distribución de Trabajo No Contributorio - Carta Balance ${ }^{\circ} 22$

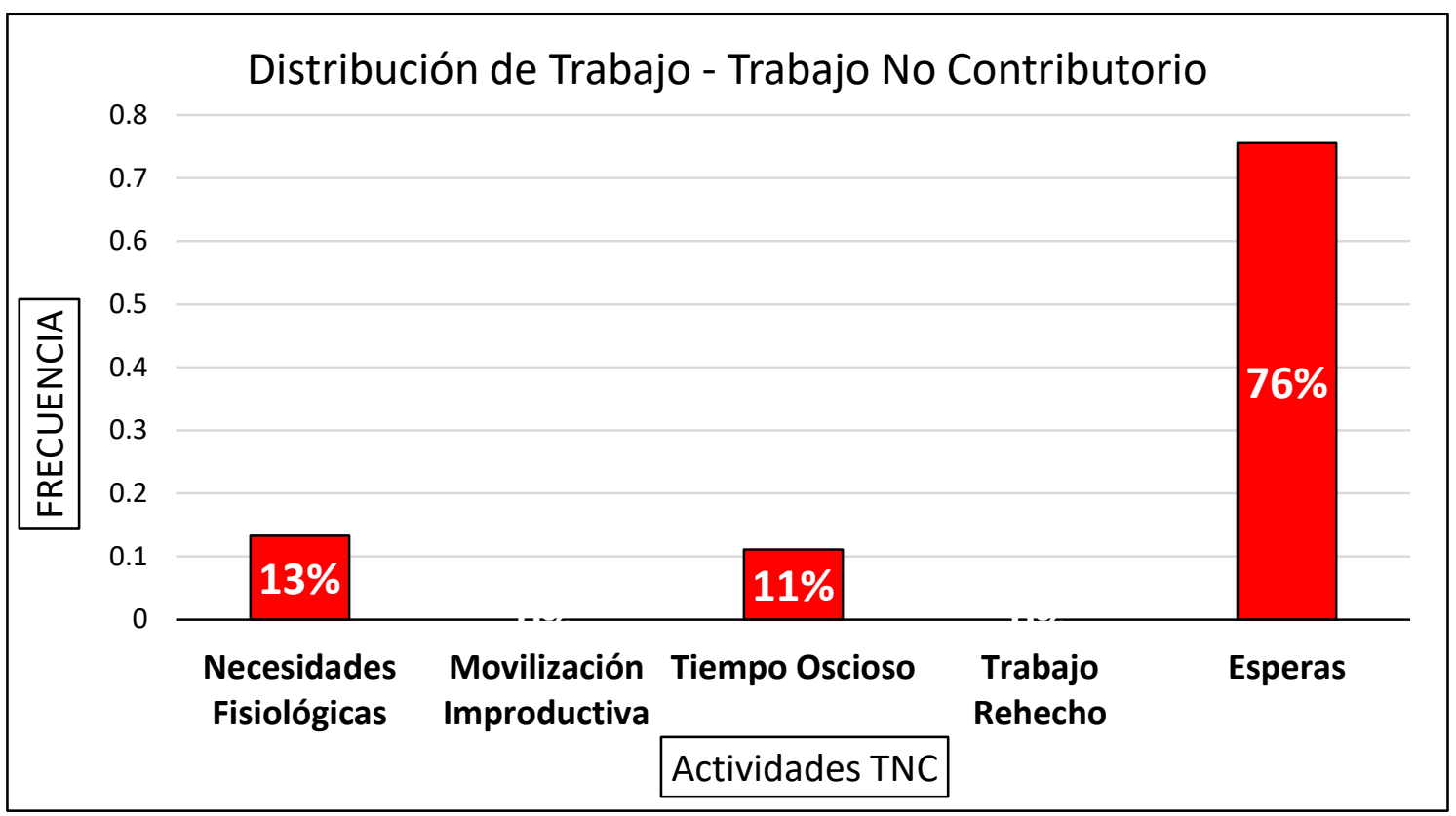

Fuente: Elaboración Propia. 
Figura No 33: Distribución Promedio de Trabajo de Enchape de Cerámico - Post Implementación de Mejoras

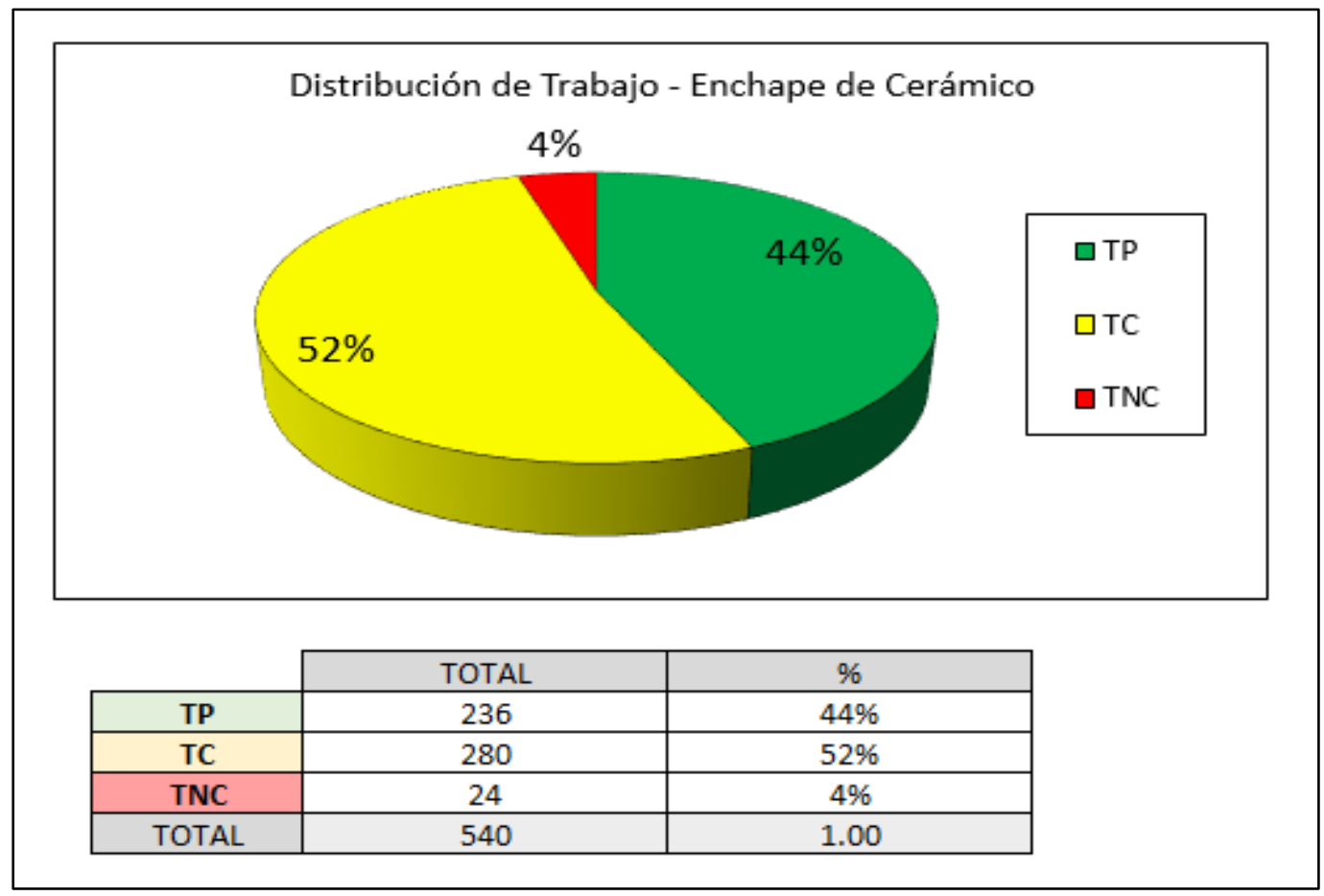

Fuente: Elaboración Propia.

Figura No 34: Distribución Promedio de Trabajo Productivo de Enchape de Cerámico Post Implementación de Mejoras

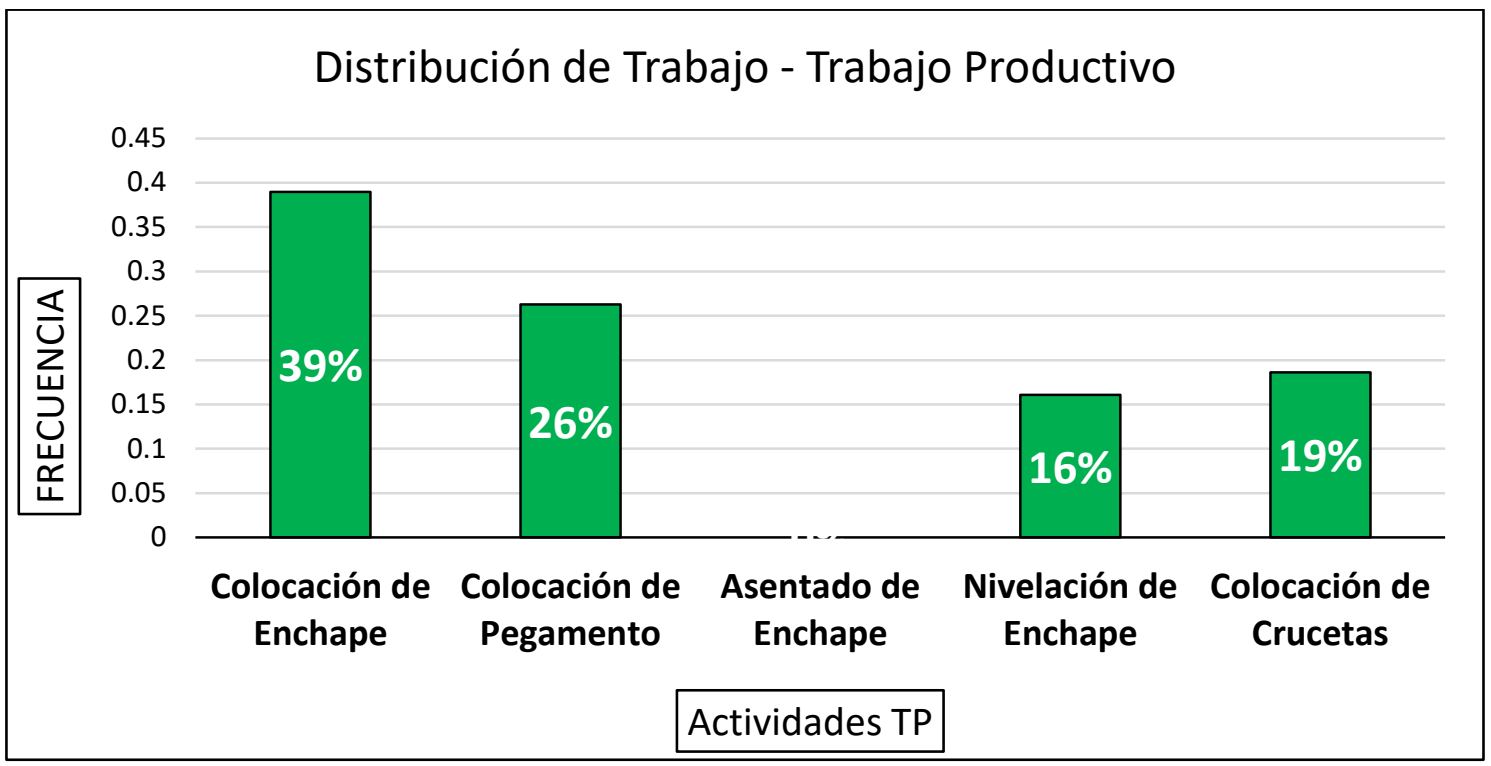

Fuente: Elaboración Propia. 
Figura No 35: Distribución Promedio de Trabajo Contributorio de Enchape de Cerámico - Post Implementación de Mejoras

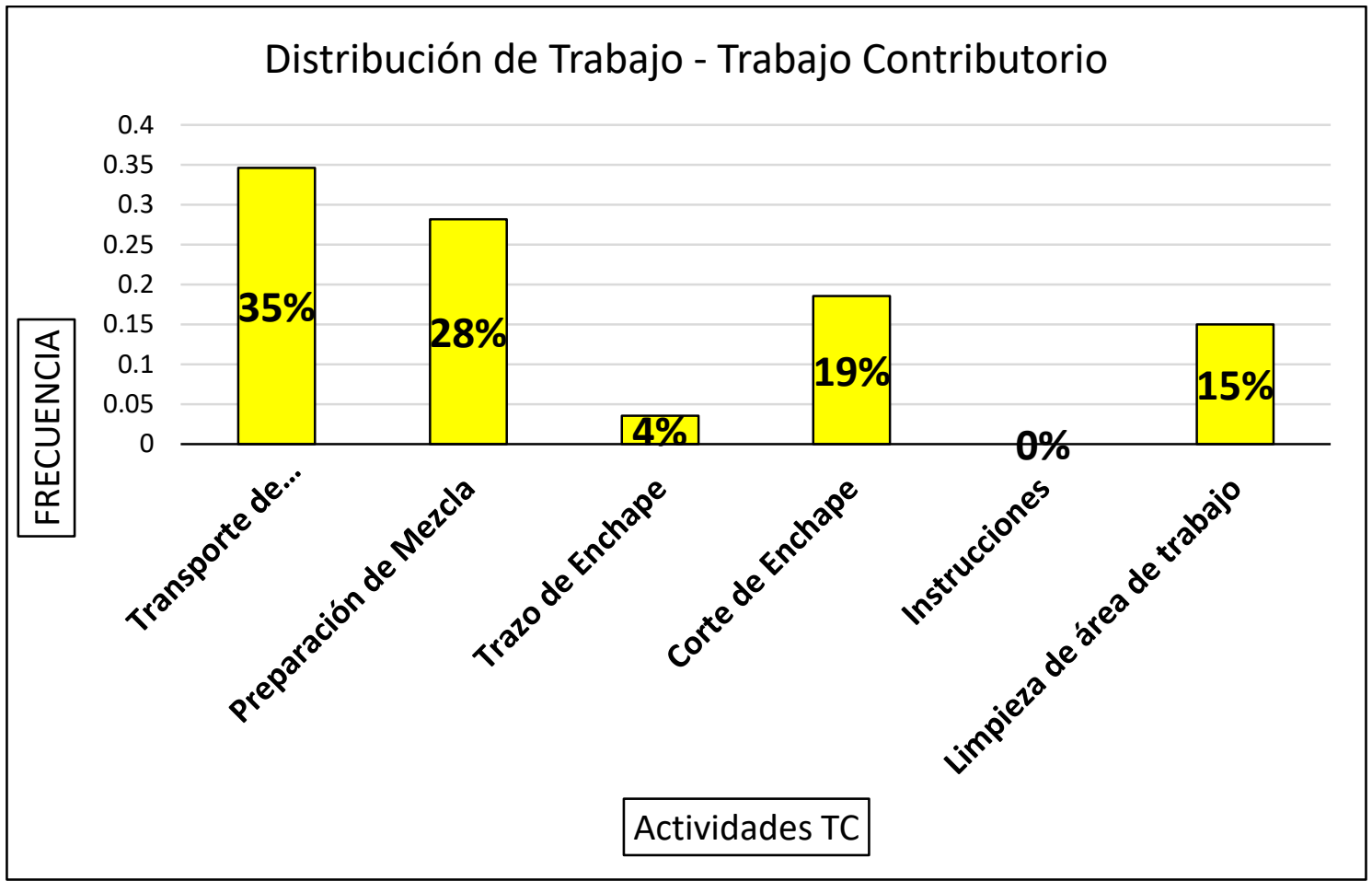

Fuente: Elaboración Propia.

Figura No 36: Distribución Promedio de Trabajo No Contributorio de Enchape de Cerámico - Post Implementación de Mejoras

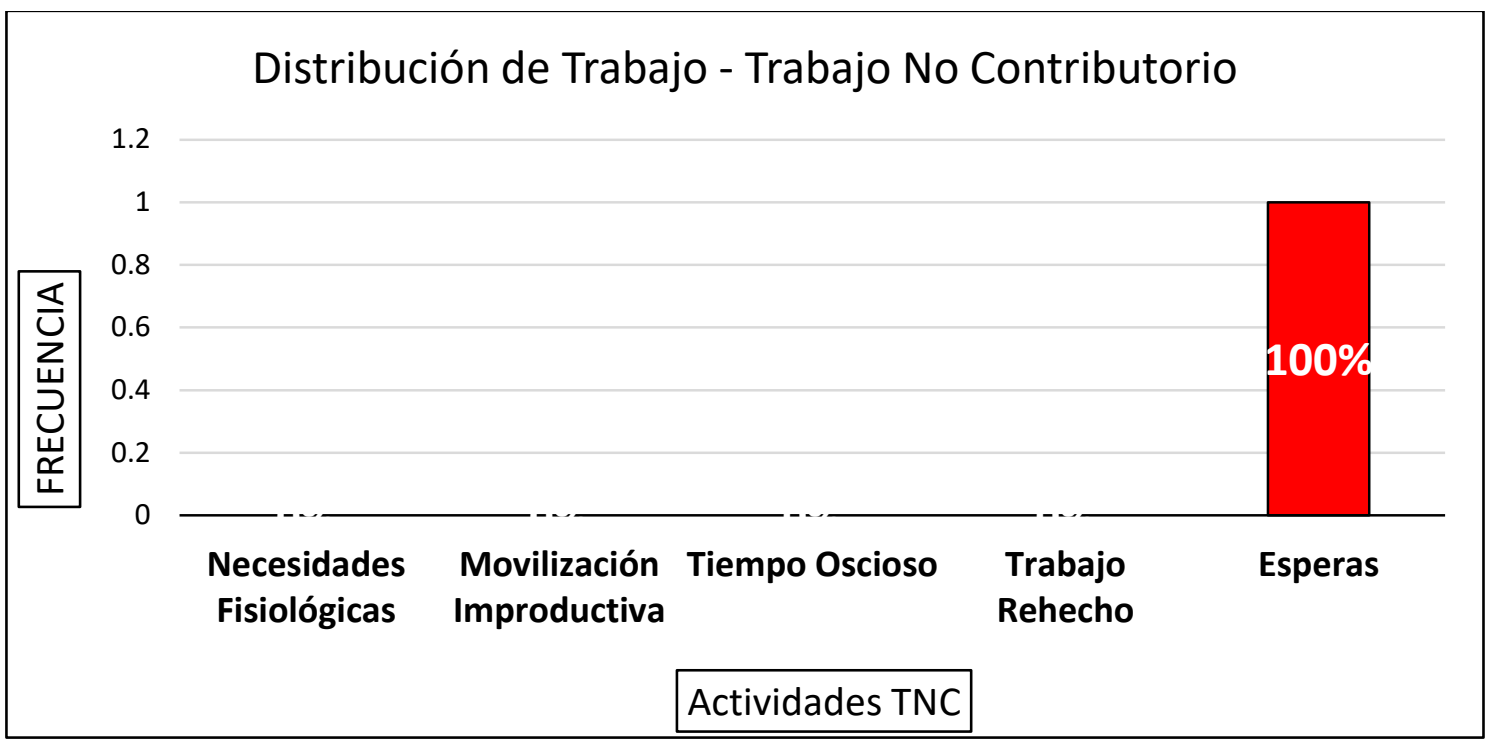

Fuente: Elaboración Propia. 
A simple vista los resultados son favorables y esto se nota haciendo una comparación simple en los TP, TC y TNC de las partidas analizadas el cual se detalla a continuación en la siguiente tabla:

Tabla Nº 10: Comparación de los TP, TC y TNC de las partidas analizadas

\begin{tabular}{|c|c|}
\hline Antes de la implementación & Después de la implementación \\
\hline $\begin{array}{l}\text { Asentado de ladrillo: } \\
\begin{array}{cl}\text { - } & \text { TP: } 35 \% \\
\text { - } & \text { TC: } 53 \% \\
\text { - } & \text { TNC: } 12 \%\end{array}\end{array}$ & $\begin{array}{l}\text { Asentado de ladrillo } \\
\begin{array}{cc}\text { - } & \text { TP: } 51 \% \\
\text { - } & \text { TC: } 42 \% \\
\text { - } & \text { TNC: } 7 \%\end{array}\end{array}$ \\
\hline 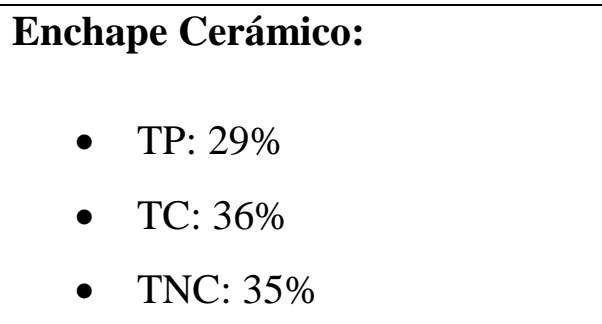 & $\begin{array}{c}\text { Enchape Cerámico } \\
\begin{array}{c}\text { - } \\
\text { - } \\
\text { - TP: } 44 \% \\
\text { - TNC: } 4 \%\end{array}\end{array}$ \\
\hline
\end{tabular}

Según la tabla anterior se observa un mejoramiento sustancial en los tres tipos de trabajo de un 35\% a 51\% del trabajo productivo en la actividad de asentado de ladrillo y de un 29\% al 44\% de trabajo productivo en la actividad de Enchape cerámico, respectivamente.

Haciendo un análisis más detallado se observa que el aumento del Trabajo Productivo (TP), fue debido que se hizo el esfuerzo en reducir el Trabajo No Contributorio (TNC) y una pequeña parte del Trabajo Contributorio (TC). 


\subsection{EVALUACIÓN ECONÓMICA DE LA PROPUESTA}

En esta sección del trabajo de investigación se realizó las estimaciones del ahorro (beneficio) y costos producidos por efecto de la propuesta de mejora. En el cuadro siguiente se muestra los recursos utilizados de manera detallada, incluido sus costos para las partidas analizadas en el trabajo de investigación, ver los cuadros $\mathrm{N}^{\circ} 36$ y 37:

Tabla $\mathrm{N}^{\mathrm{o}}$ 11: Estimado de costos antes y después de la implementación de la mejora Asentado de Ladrillo Blanco

\begin{tabular}{|c|c|c|c|c|c|c|c|}
\hline \multicolumn{8}{|c|}{ ASENTADO DE LADRILLO BLANCO - ANTES DE LA IMPLEMENTACION } \\
\hline Operarios & 3 & 3 & 3 & 3 & 3 & 3 & \\
\hline S/. Operario por Día Prom. & S/ 144.50 & S/ 144.50 & S/ 144.50 & S/ 144.50 & S/ 144.50 & S/ 93.50 & \\
\hline Ayudantes & 2 & 2 & 2 & 2 & 2 & 2 & \\
\hline S/. Ayudante por Día Prom. & S/ 93.50 & s/ 93.50 & S/ 93.50 & S/ 93.50 & S/ 93.50 & S/ 60.50 & \\
\hline TOTAL & S/ 620.50 & s/ 620.50 & s/ 620.50 & s/ 620.50 & S/ 620.50 & S/ 401.50 & S/ $3,504.00$ \\
\hline \multicolumn{8}{|c|}{ ASENTADO DE LADRILLO BLANCO - DESPUES DE LA IMPLEMENTACION } \\
\hline DÍAS & LUNES & MARTES & MIÉRCOLES & JUEVES & TOTAL & & \\
\hline Practicante & s/ 300.00 & & & & & & \\
\hline Operarios & 3 & 3 & 3 & 3 & & & \\
\hline S/. Operario por Día Prom. & S/ 144.50 & s/ 144.50 & S/ 144.50 & S/ 144.50 & & & \\
\hline Ayudantes & 2 & 2 & 2 & 2 & & & \\
\hline S/. Ayudante por Día Prom. & s/ 93.50 & 93.50 & s/ 93.50 & s/ 93.50 & & & \\
\hline TOTAL & s/ 920.50 & s/ 620.50 & s/ 620.50 & s/ 620.50 & $s / 2,782.00$ & & \\
\hline
\end{tabular}

Fuente: Elaboración Propia.

Tabla $\mathrm{N}^{\circ}$ 12: Estimado de costos antes y después de la implementación de la mejora Enchape de Cerámico

\begin{tabular}{|c|c|c|c|c|c|c|c|c|c|c|c|c|}
\hline \multicolumn{13}{|c|}{ ENCHAPE DE CERAMICO - ANTES DE LA IMPLEMENTACION } \\
\hline DÍAS & & UNES & & IARTES & & ÉRCOLES & & UEVES & & IERNES & SÁBADO & \\
\hline Operarios & & 1 & & 1 & & 1 & & 1 & & 1 & 1 & \\
\hline S/. Operario por Dfa Prom. & & 144.50 & & 144.50 & & 144.50 & & 144.50 & s/ & 144.50 & s/ 93.50 & \\
\hline Ayudantes & & 1 & & 1 & & 1 & & 1 & & 1 & 1 & \\
\hline S/. Ayudante por Día Prom. & & 93.50 & & 93.50 & & 93.50 & & 93.50 & & 93.50 & s/ 60.50 & \\
\hline TOTAL & & 238.00 & s & 238.00 & s, & 238.00 & s) & 238.00 & s/ & 238.00 & s/ 154.00 & S/ $1,344.00$ \\
\hline \multicolumn{13}{|c|}{ ENCHAPE DE CERAMICO - DESPUES DE LA IMPLEMENTACION } \\
\hline DÍAS & & UNES & & IARTES & & ÉRCOLES & & & & & & \\
\hline Practicante & & 300.00 & & & & & & & & & & \\
\hline Operarios & & 1 & & 1 & & 1 & & & & & & \\
\hline S/. Operario por Día Prom. & & 144.50 & & 144.50 & & 144.50 & & & & & & \\
\hline Ayudantes & & 1 & & 1 & & 1 & & & & & & \\
\hline S/. Ayudante por Día Prom. & s) & 93.50 & & 93.50 & & 93.50 & & & & & & \\
\hline TOTAL & s) & 238.00 & $\mathbf{s}$ & 238.00 & $\mathbf{s}$ & 238.00 & s) & 714.00 & & & & \\
\hline
\end{tabular}

Fuente: Elaboración Propia. 


\section{CAPÍTULO V: CONCLUSIONES}

- La medición y el análisis del rendimiento de los trabajos, mediante el uso de la metodología del ciclo de mejoramiento de la productividad, fue de gran ayuda para determinar el estado actual de las partidas analizadas. Se logró identificar el porcentaje de tiempo que se emplea en los trabajos productivos, contributorios y no contributorios, los problemas existentes en la ejecución de estas partidas. De esta manera, se implementó las mejoras y se midió los resultados de esta implementación, tal como señala en la Figura Nº 37.

Figura No 37: TP, TC y TCN antes y después de la implementación de la mejora

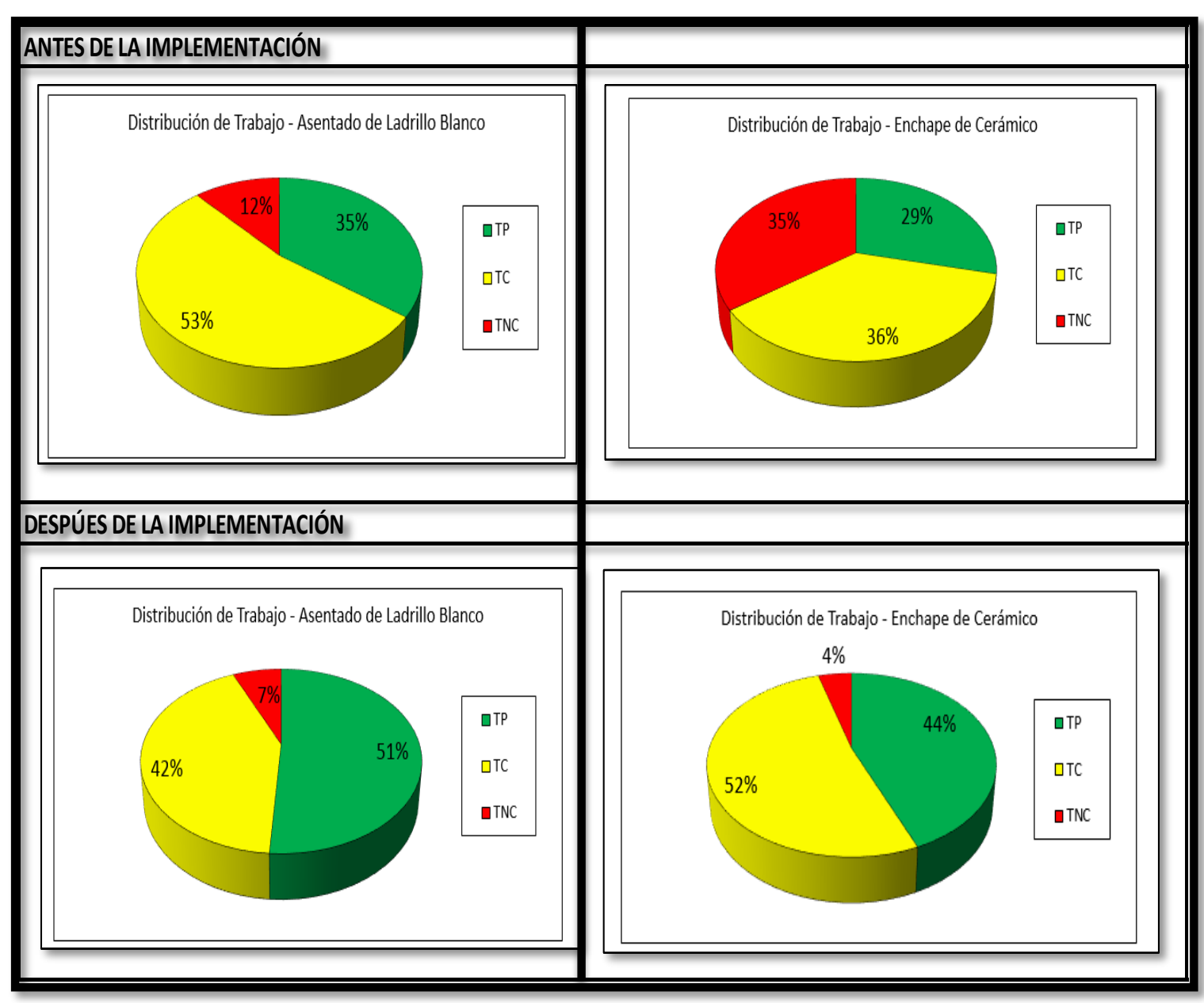

Fuente: Elaboración Propia.

Según el gráfico $\mathrm{N}^{\circ}$ 37, se observa una disminución del 5\% y 31\% de Trabajo No Contributorio (TNC) en las partidas de Asentado de ladrillo y Enchape de 
Cerámico, respectivamente. Con esto se puede concluir que la implementación de las mejoras resultó favorablemente en la reducción de TNC y el aumento del TP que se observa en las dos partidas analizadas.

- Las herramientas de Last Planner fue de gran aporte al proyecto, puesto que las reuniones que se establecieron para identificar y eliminar restricciones aportaron a la mejora de la productividad en las partidas evaluadas. El principal problema que existía en estas partidas era la existencia de restricciones y la falta de coordinaciones entre las contratistas, por lo que las reuniones Last Planner aportaron a la organización de estas partidas y establecer un horizonte de trabajo colaborativo para llegar a la meta del proyecto.

Como contribución, después de la implementación, en el caso de estudio, desarrollamos un proceso para el incremento de la productividad, ver Figura $\mathrm{N}^{\circ}$ 38, que se puede extender no solo a las partidas analizadas, sino que también a todas las partidas en general, iniciando un proceso de estandarización.

Figura No 38: Proceso Propuesto para Incremento de la Productividad.

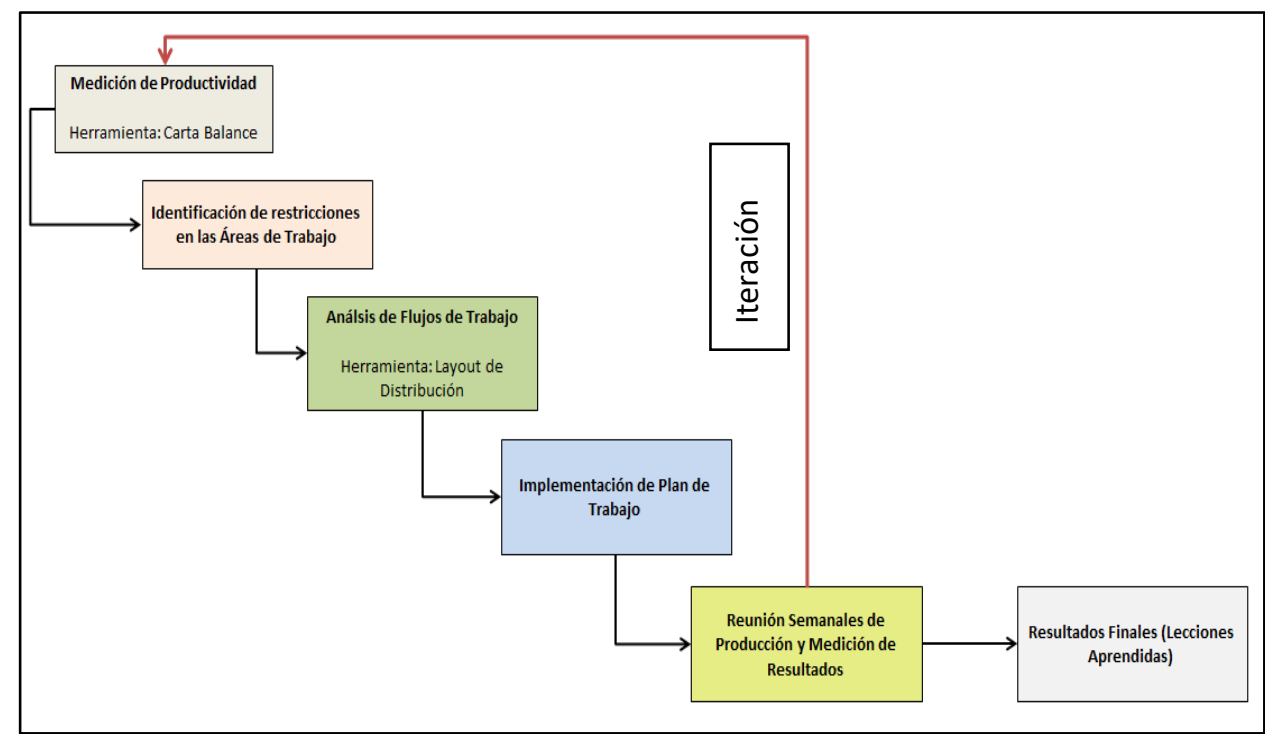

La implementación de esta mejora fue sustancial para la obra, los resultados se reflejaron en un aumento de la productividad, los cuales se puede observar en la figura $\mathrm{N}^{\circ}$ 37. Este proceso ha probado tener resultados positivos, con lo cual puede ser implementado para otras partidas de la obra. 
- Se identificó los costos acarreados antes y después de la implementación de la mejora los cuales se detallan en las tablas $\mathrm{N}^{\circ} 36 \mathrm{y} \mathrm{N}^{\circ} 37$, de los cuales se concluye que se obtiene un ahorro en costo del orden del 20\% en la partida de Asentado de Ladrillo y aproximadamente $45 \%$ de ahorro en costo en la partida de Enchape de cerámico. Con esto se demuestra que tanto la productividad y costos de obra están estrechamente ligados. 


\section{CAPÍTULO VI: RECOMENDACIONES}

- Las inclusiones de indicadores de medición de la productividad son de vital importancia en las obras del sector construcción, debido a que esto nos permite conocer en qué tipo de trabajo (TP, TC, TNC) se ocupa mayor porcentaje del tiempo.

- Realizar las mediciones de producción en un lapso mínimo de una hora. Además, es recomendable que estos se realicen a media mañana y a mitad de la tarde, debido a que en este momento ya se tienen a todas las cuadrillas con la producción promedio.

- Se recomienda que las reuniones semanales tengan una duración máxima de dos horas.

- Se recomienda desarrollar el flujo de trabajo que se tiene entre las partidas más importantes, para ello se pueden apoyar en un diagrama de Pareto, debido a que gran parte de las pérdidas se encuentran dentro de estos flujos y no en los procesos terminados; por ejemplo, el transporte de materiales, instrucciones, transmisión de información, etc. 


\section{CAPÍTULO VII: BIBLIOGRAFÍA}

Mejía, G., \& Hernández, C. (2007). Seguimiento de la Productividad en Obra: Técnicas de Medición de Rendimiento de Mano de Obra. (F. d. Fisicomecánicas, Ed.) UIS Ingenierías, 6(2), 45-59.

Botero, L., \& Álvarez, M. (2003). Identificación de Pérdidas en el Proceso Productivo de la Construcción. Revista Universidad EAFIT(130), 65-78.

Bustamante Ajahuana, A. F. (2018). Optimización de la productividad y los costos mediante la aplicación de Lean Construction, en la construcción de falso piso 1:8 e=4; Proyecto: SNIP 67018 Ilo, Moquegua 2018. Perú: Escuela de Postgrado Universidad César Vallejo.

Enshassi, A. (2007). Benchmarking masonry labor productivity. International Journal of Productivity and Performance Management, 56(4), 358-368.

Adnan, E. (2013). Tendencias para optimizar la productividad en los proyectos de construcción en Palestina. Revista Ingeniería de Construcción RIC, 28(2), 173206.

IEDEP. (2018). Proyecciones Macroeconómicas 2018-2019. Obtenido de https://www.camaralima.org.pe/repositorioaps/0/0/par/proyeccionesmacro/iedep \%20proyecciones\%200718.pdf

Morales, N., \& Galeas, J. (2006). Diagnóstico y Evaluación de la relación entre el grado de industrialización y los sistemas de gestión con el nivel de productividad en obras de construcción. Lima, Perú: PUCP.

Ponz-Tienda, J. L. (2014). Labor Productivity in the Construction Industry - Factors Influencing the Spanish Construction Labor. International Scholarly and Scientific Research \& Innovation, 8(10), 1061-1070.

Santana, J. M. (1989). El tiempo improductivo en obras de construcción. Revista Ingeniería de Construcción(7).

Serpell, A. (2002). Administración de Operaciones de Construcciones. Santiago de Chile: ALFAOMEGA.

Ghio, V. (2001). Productividad en Obras de Construcción (1 ed.). Lima, Perú: PUCP Fondo Editorial.

Gómez, A., \& Morales, D. (2016). Análisis de la Productividad en la Construcción de Vivienda basada en Rendimientos de Mano de Obra. INGE CUC, 12(1), 21-31. 
Botero, L. (2004). Construcción Sin Pérdidas. Colombia: Legis S.A.

Harris, F., \& McCaffer, R. (2005). Construction Management: Manual de Gestión de Proyecto y Dirección de Obra. Barcelona: Editorial Gustavo Gili, S.L.

Abdul, M., Vamsi, B., \& Ranjith, V. (2014). APPLICATION AND ANALYSIS OF LAST PLANNER SYSTEM IN THE CONSTRUCTION INDUSTRY. International Journal of Research in Engineering \& Technology, 33-44.

Ballard, G. H. (Mayo de 2000). The Last Planner System of Production Control. The University of Birmingham. Obtenido de http://www.leanconstruction.dk/media/15590/ballard2000-dissertation.pdf

Pellicer, E., Cerveró, F., Lozano, A., \& Ponz, J. L. (2015). THE LAST PLANNER SYSTEM OF CONSTRUCTION PLANNING AND CONTROL AS A TEACHING AND LEARNING TOOL. Proceedings of INTED2015 Conference, 4877-4884. Obtenido de https://www.researchgate.net/publication/273129218_THE_LAST_PLANNER_ SYSTEM_OF_CONSTRUCTION_PLANNING_AND_CONTROL_AS_A_TE ACHING_AND_LEARNING_TOOL

Itodo, E., Pasquire, C., \& Ameh, O. (2014). THE MAGIC OF THE LAST PLANNER ${ }^{\circledR}$ SYSTEM FOR NIGERIAN CONSTRUCTION. Proceedings IGLC-22, 605-616. Obtenido https://www.researchgate.net/publication/265013647_THE_MAGIC_OF_THE_ LAST_PLANNER_R_SYSTEM_FOR_NIGERIAN_CONSTRUCTION

Ballard, G., \& Howell, G. (2003). An Update on Last Planner. IGLC 11. Obtenido de https://www.researchgate.net/publication/254576025_AN_UPDATE_ON_LAS T_PLANNER1

Hamzeh, F., Ballard, G., \& Tommelein, I. (2012). Rethinking Lookahead Planning to Optimize Construction Workflow. Lean Construction Journal 2012, 15-34. Obtenido de https://www.leanconstruction.org/media/library/id45/Rethinking_Lookahead_Pl anning_to_Optimize_Construction_Workflow.pdf

Rodriguez, A., Alarcón, L., \& Pellicer, E. (2013). La gestión de la obra desde la perspectiva del último planificador. Revista de Obras Públicas(3518), 35-44. Obtenido de https://www.researchgate.net/publication/318682002_La_gestion_de_la_obra_d esde_la_perspectiva_del_ultimo_planificador

Kalsaas, B., Grindheim, I., \& Læknes, N. (2014). Integrated Planning vs Last Planner System. Proceedings IGLC-22, 639-650. Obtenido de 
https://www.researchgate.net/publication/280922309_INTEGRATED_PLANNI NG_VS_LAST_PLANNER_SYSTEM

Wendkos, D. E. (1992). Desarrollo Humano. Mexico: Mc. Graw Hill.

Heinrich, C. M. (2018). Negocio Inmobiliario. Lima, Lima, Perú: UPC. 


\section{CAPÍTULO VI: ANEXOS}

Tabla No 13: Carta Balance ${ }^{\circ} 1$ - Asentado de Ladrillo

\begin{tabular}{|c|c|c|c|c|c|}
\hline $\mathrm{N}^{0}$ & Obrero\#1 & Obrero\#2 & Obrero\#3 & Obrero \#4 & Obrero\#5 \\
\hline 1 & NA & NA & NA & TH & $\mathrm{CL}$ \\
\hline 2 & NA & NA & NA & TH & $\mathrm{CL}$ \\
\hline 3 & PM & NA & NA & TO & $\mathrm{CL}$ \\
\hline 4 & PM & NA & NA & $\mathrm{TH}$ & $\mathrm{TH}$ \\
\hline 5 & TO & NA & NA & $\mathrm{TH}$ & $\mathrm{CL}$ \\
\hline 6 & PM & PM & NA & $\mathrm{TH}$ & $\mathrm{CL}$ \\
\hline 7 & PM & PM & PM & $T H$ & $\mathrm{CL}$ \\
\hline 8 & $\mathrm{CM}$ & $\mathrm{CM}$ & $E$ & $\mathrm{TH}$ & $\mathrm{TH}$ \\
\hline 9 & $\mathrm{CM}$ & $\mathrm{CM}$ & $E$ & L & $\mathrm{CL}$ \\
\hline 10 & NA & $\mathrm{CM}$ & $E$ & $E$ & $\mathrm{CL}$ \\
\hline 11 & $\mathrm{NA}$ & AL & $E$ & $\mathrm{TH}$ & $\mathrm{CL}$ \\
\hline 12 & NA & AL & NA & TH & $\mathrm{TH}$ \\
\hline 13 & $\mathrm{CM}$ & AL & NA & $\mathrm{TH}$ & ML \\
\hline 14 & $\mathrm{CM}$ & AL & $E$ & $\mathrm{TH}$ & $\mathrm{CL}$ \\
\hline 15 & AL & AL & $\mathrm{CM}$ & $\mathrm{TH}$ & $\mathrm{CL}$ \\
\hline 16 & AL & AL & $\mathrm{CM}$ & $\mathrm{TH}$ & $\mathrm{CL}$ \\
\hline 17 & AL & NA & $\mathrm{CM}$ & $\mathrm{TH}$ & $\mathrm{CL}$ \\
\hline 18 & AL & NA & $\mathrm{CM}$ & TH & $\mathrm{CL}$ \\
\hline 19 & NA & NA & $\mathrm{CM}$ & $\mathrm{TH}$ & $\mathrm{TH}$ \\
\hline 20 & NA & NA & $\mathrm{CM}$ & $\mathrm{TH}$ & ML \\
\hline 21 & NA & NA & $\mathrm{CM}$ & $\mathrm{TH}$ & $\mathrm{CL}$ \\
\hline 22 & NA & PM & AL & $\mathrm{TH}$ & ML \\
\hline 23 & NA & $C A$ & AL & $\mathrm{TH}$ & ML \\
\hline 24 & PM & NA & NA & $\mathrm{TH}$ & $\mathrm{CL}$ \\
\hline 25 & $C A$ & $C A$ & NA & TH & $\mathrm{CL}$ \\
\hline 26 & $C A$ & $\mathrm{CH}$ & AL & $\mathrm{TH}$ & $\mathrm{CL}$ \\
\hline 27 & NA & 1 & $\mathrm{CM}$ & $\mathrm{TH}$ & $\mathrm{CL}$ \\
\hline 28 & NA & PM & $\mathrm{CM}$ & $\mathrm{TH}$ & $\mathrm{CL}$ \\
\hline 29 & NA & $\mathrm{CM}$ & $E$ & $\mathrm{TH}$ & $\mathrm{CL}$ \\
\hline 30 & NA & $\mathrm{AL}$ & $\mathrm{CM}$ & $\mathrm{TH}$ & $\mathrm{CL}$ \\
\hline 31 & PM & $\mathrm{AL}$ & $\mathrm{AL}$ & $\mathrm{CL}$ & $\mathrm{CL}$ \\
\hline 32 & AL & AL & NA & $\mathrm{CL}$ & $\mathrm{CL}$ \\
\hline 33 & NA & AL & NA & $\mathrm{CL}$ & ML \\
\hline 34 & $\mathrm{CM}$ & AL & AL & $\mathrm{TH}$ & I \\
\hline 35 & $E$ & NA & AL & $\mathrm{TH}$ & $\mathrm{CL}$ \\
\hline 36 & $\mathrm{AL}$ & NA & $E$ & $\mathrm{CL}$ & $\mathrm{CL}$ \\
\hline 37 & AL & PM & $\mathrm{AL}$ & $\mathrm{TH}$ & $\mathrm{CL}$ \\
\hline 38 & $\mathrm{AL}$ & PM & NA & $\mathrm{TH}$ & I \\
\hline 39 & $\mathrm{AL}$ & $C A$ & I & TH & ML \\
\hline 40 & NA & $C A$ & $E$ & TH & $\mathrm{CL}$ \\
\hline 41 & NA & $C A$ & $E$ & $\mathrm{TH}$ & $\mathrm{CL}$ \\
\hline 42 & $C A$ & $\mathrm{CH}$ & NA & TH & ML \\
\hline 43 & $C A$ & $\mathrm{CH}$ & $\mathrm{NA}$ & $\mathrm{TH}$ & $\mathrm{TH}$ \\
\hline 44 & $\mathrm{CH}$ & PM & $\mathrm{AL}$ & $\mathrm{TH}$ & $\mathrm{CL}$ \\
\hline 45 & PM & $\mathrm{CM}$ & NA & $\mathrm{TH}$ & $\mathrm{CL}$ \\
\hline
\end{tabular}

\begin{tabular}{|c|c|c|c|c|c|}
\hline 46 & $\mathrm{CM}$ & $\mathrm{CM}$ & $\mathrm{NA}$ & $\mathrm{TH}$ & $\mathrm{TH}$ \\
\hline 47 & $\mathrm{NA}$ & $\mathrm{NA}$ & $\mathrm{NA}$ & $\mathrm{E}$ & $\mathrm{CL}$ \\
\hline 48 & $\mathrm{AL}$ & $\mathrm{AL}$ & $\mathrm{I}$ & $\mathrm{E}$ & $\mathrm{ML}$ \\
\hline 49 & $\mathrm{NA}$ & $\mathrm{AL}$ & $\mathrm{AL}$ & $\mathrm{CL}$ & $\mathrm{CL}$ \\
\hline 50 & $\mathrm{AL}$ & $\mathrm{AL}$ & $\mathrm{NA}$ & $\mathrm{CL}$ & $\mathrm{CL}$ \\
\hline 51 & $\mathrm{NA}$ & $\mathrm{NA}$ & $\mathrm{NA}$ & $\mathrm{CL}$ & $\mathrm{CL}$ \\
\hline 52 & $\mathrm{TH}$ & $\mathrm{NA}$ & $\mathrm{AL}$ & $\mathrm{CL}$ & $\mathrm{TH}$ \\
\hline 53 & $\mathrm{AL}$ & $\mathrm{NA}$ & $\mathrm{NA}$ & $\mathrm{TH}$ & $\mathrm{TH}$ \\
\hline 54 & $\mathrm{NA}$ & $\mathrm{NA}$ & $\mathrm{NA}$ & $\mathrm{CL}$ & $\mathrm{CL}$ \\
\hline 55 & $\mathrm{NA}$ & $\mathrm{PM}$ & $\mathrm{NA}$ & $\mathrm{TH}$ & $\mathrm{CL}$ \\
\hline 56 & $\mathrm{PM}$ & $\mathrm{CA}$ & $\mathrm{NA}$ & $\mathrm{E}$ & $\mathrm{CL}$ \\
\hline 57 & $\mathrm{CA}$ & $\mathrm{CA}$ & $\mathrm{NA}$ & $\mathrm{I}$ & $\mathrm{CL}$ \\
\hline 58 & $\mathrm{CA}$ & $\mathrm{CM}$ & $\mathrm{NA}$ & $\mathrm{TH}$ & $\mathrm{CL}$ \\
\hline 59 & $\mathrm{NA}$ & $\mathrm{CM}$ & $\mathrm{E}$ & $\mathrm{E}$ & $\mathrm{CL}$ \\
\hline 60 & $\mathrm{CM}$ & $\mathrm{NA}$ & $\mathrm{NA}$ & $\mathrm{TH}$ & $\mathrm{ML}$ \\
\hline 61 & $\mathrm{NA}$ & $\mathrm{AL}$ & $\mathrm{NA}$ & $\mathrm{TH}$ & $\mathrm{CL}$ \\
\hline 62 & $\mathrm{NA}$ & $\mathrm{AL}$ & $\mathrm{PM}$ & $\mathrm{TH}$ & $\mathrm{ML}$ \\
\hline 63 & $\mathrm{AL}$ & $\mathrm{AL}$ & $\mathrm{CA}$ & $\mathrm{TH}$ & $\mathrm{E}$ \\
\hline 64 & $\mathrm{NA}$ & $\mathrm{AL}$ & $\mathrm{CA}$ & $\mathrm{TH}$ & $\mathrm{TH}$ \\
\hline 65 & $\mathrm{PM}$ & $\mathrm{NA}$ & $\mathrm{CA}$ & $\mathrm{TH}$ & $\mathrm{CL}$ \\
\hline 66 & $\mathrm{CA}$ & $\mathrm{NA}$ & $\mathrm{CA}$ & $\mathrm{E}$ & $\mathrm{TH}$ \\
\hline 67 & $\mathrm{~L}$ & $\mathrm{NA}$ & $\mathrm{CA}$ & $\mathrm{TH}$ & $\mathrm{ML}$ \\
\hline 68 & $\mathrm{~L}$ & $\mathrm{PM}$ & $\mathrm{CA}$ & $\mathrm{CL}$ & $\mathrm{CL}$ \\
\hline 69 & $\mathrm{E}$ & $\mathrm{CA}$ & $\mathrm{CA}$ & $\mathrm{TH}$ & $\mathrm{CL}$ \\
\hline 70 & $\mathrm{TH}$ & $\mathrm{CA}$ & $\mathrm{PM}$ & $\mathrm{E}$ & $\mathrm{CL}$ \\
\hline 71 & $\mathrm{MI}$ & $\mathrm{CA}$ & $\mathrm{CA}$ & $\mathrm{E}$ & $\mathrm{CL}$ \\
\hline 72 & $\mathrm{Ml}$ & $\mathrm{CH}$ & $\mathrm{NA}$ & $\mathrm{TH}$ & $\mathrm{CL}$ \\
\hline 73 & $\mathrm{AV}$ & $\mathrm{PM}$ & $\mathrm{CA}$ & $\mathrm{TH}$ & $\mathrm{CL}$ \\
\hline 74 & $\mathrm{AV}$ & $\mathrm{CM}$ & $\mathrm{CM}$ & $\mathrm{TH}$ & $\mathrm{CL}$ \\
\hline 75 & $\mathrm{CM}$ & $\mathrm{AL}$ & $\mathrm{AL}$ & $\mathrm{E}$ & $\mathrm{TH}$ \\
\hline 76 & $\mathrm{NA}$ & $\mathrm{NA}$ & $\mathrm{NA}$ & $\mathrm{TH}$ & $\mathrm{ML}$ \\
\hline 77 & $\mathrm{AL}$ & $\mathrm{AL}$ & $\mathrm{AL}$ & $\mathrm{CL}$ & $\mathrm{TH}$ \\
\hline 78 & $\mathrm{AL}$ & $\mathrm{AL}$ & $\mathrm{AL}$ & $\mathrm{CL}$ & $\mathrm{CL}$ \\
\hline 79 & $\mathrm{AL}$ & $\mathrm{AL}$ & $\mathrm{AL}$ & $\mathrm{ML}$ & $\mathrm{TH}$ \\
\hline 80 & $\mathrm{AL}$ & $\mathrm{NA}$ & $\mathrm{NA}$ & $\mathrm{CL}$ & $\mathrm{ML}$ \\
\hline 81 & $\mathrm{NA}$ & $\mathrm{NA}$ & $\mathrm{NA}$ & $\mathrm{ML}$ & $\mathrm{ML}$ \\
\hline 82 & $\mathrm{NA}$ & $\mathrm{NA}$ & $\mathrm{NA}$ & $\mathrm{CL}$ & $\mathrm{CL}$ \\
\hline 83 & $\mathrm{NA}$ & $\mathrm{AV}$ & $\mathrm{NA}$ & $\mathrm{TH}$ & $\mathrm{TH}$ \\
\hline 84 & $\mathrm{CA}$ & $\mathrm{AV}$ & $\mathrm{NA}$ & $\mathrm{TH}$ & $\mathrm{CL}$ \\
\hline 85 & $\mathrm{CA}$ & $\mathrm{PM}$ & $\mathrm{CA}$ & $\mathrm{TH}$ & $\mathrm{ML}$ \\
\hline 86 & $\mathrm{AV}$ & $\mathrm{CA}$ & $\mathrm{CA}$ & $\mathrm{TH}$ & $\mathrm{CL}$ \\
\hline 87 & $\mathrm{AV}$ & $\mathrm{CM}$ & $\mathrm{NA}$ & $\mathrm{TH}$ & $\mathrm{CL}$ \\
\hline 88 & $\mathrm{AV}$ & $\mathrm{NA}$ & $\mathrm{AL}$ & $\mathrm{L}$ & $\mathrm{CL}$ \\
\hline 89 & $\mathrm{NA}$ & $\mathrm{L}$ & $\mathrm{AL}$ & $\mathrm{L}$ & $\mathrm{CL}$ \\
\hline 90 & $\mathrm{PM}$ & $\mathrm{L}$ & $\mathrm{AL}$ & $\mathrm{L}$ & $\mathrm{CL}$ \\
\hline & & & & & \\
\hline
\end{tabular}

Fuente: Elaboración Propia. 
Figura $N^{\circ}$ 39: Distribución de Trabajo de Asentado de Ladrillo - Carta Balance ${ }^{\circ} 1$

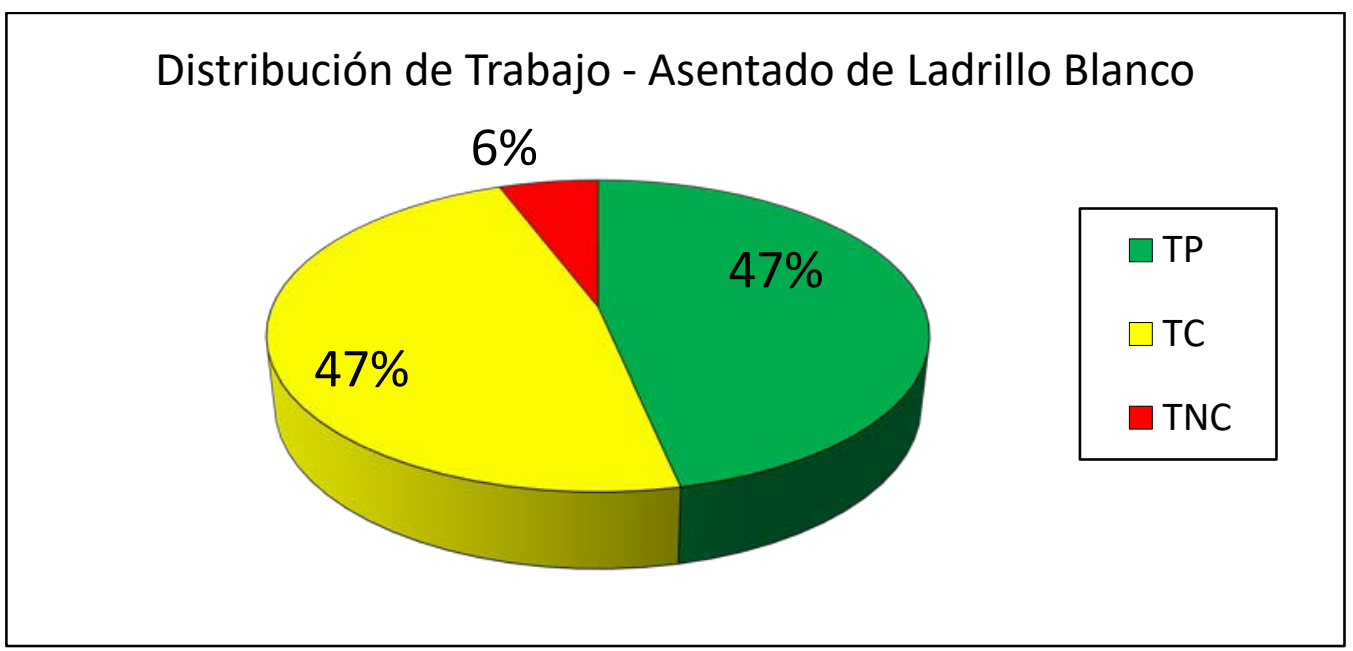

Fuente: Elaboración Propia.

Figura $N^{\circ} 40$ : Distribución de Trabajo Productivo - Carta Balance ${ }^{\circ} 1$

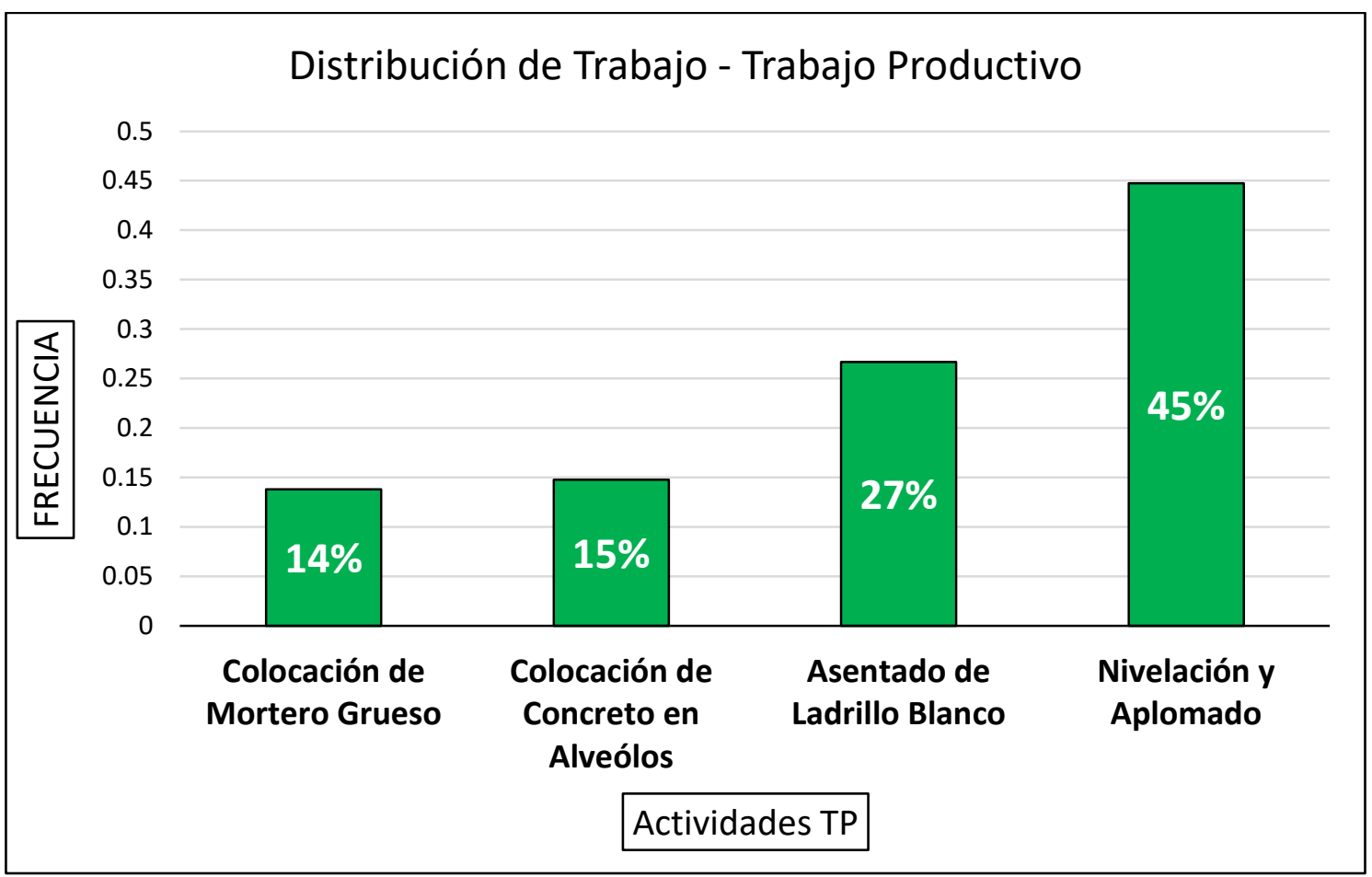

Fuente: Elaboración Propia. 
Figura No 41: Distribución de Trabajo Contributorio - Carta Balance ${ }^{\circ} 1$

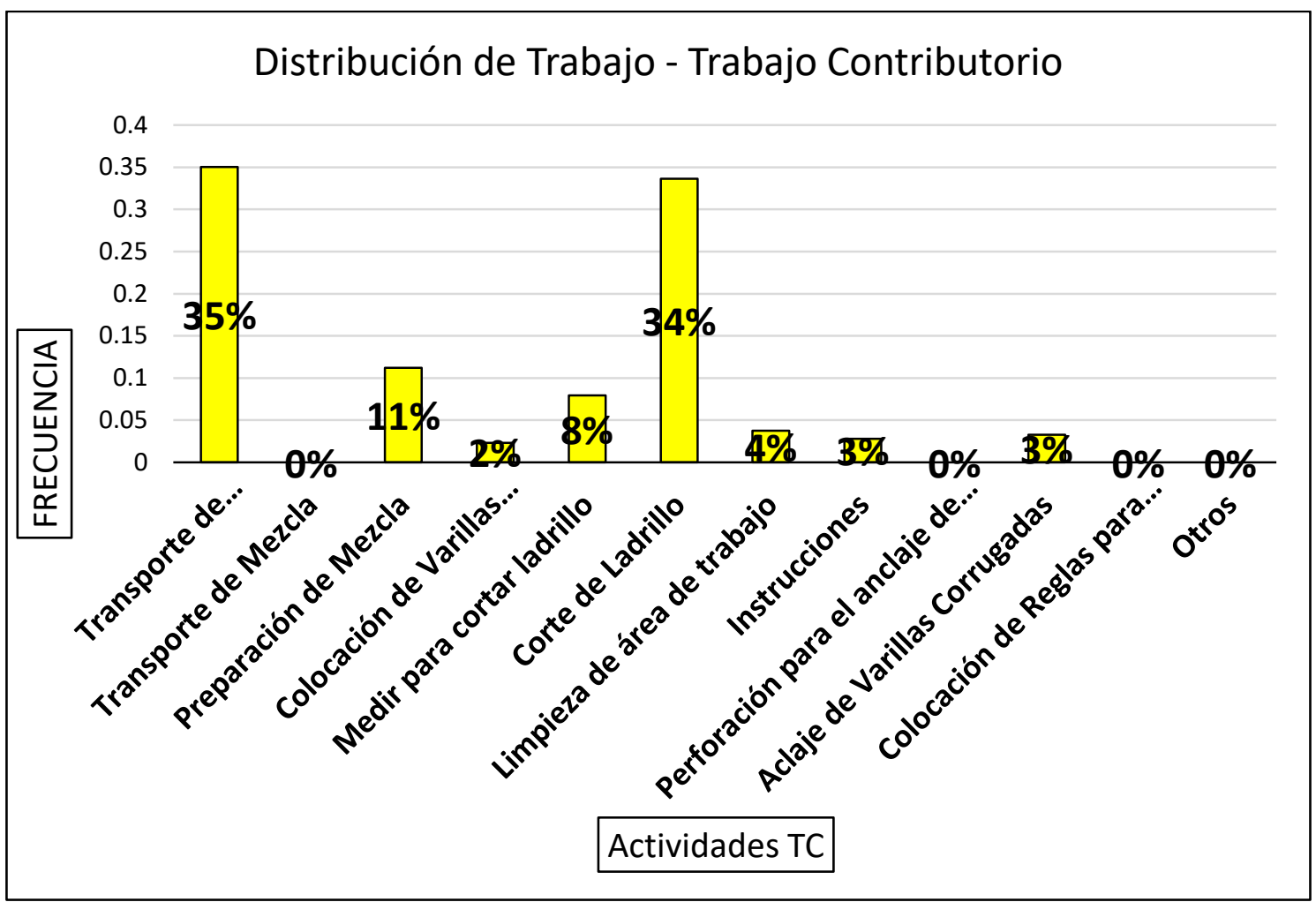

Fuente: Elaboración Propia.

Figura $N^{\circ} 42$ : Distribución de Trabajo No Contributorio - Carta Balance ${ }^{\circ} 1$

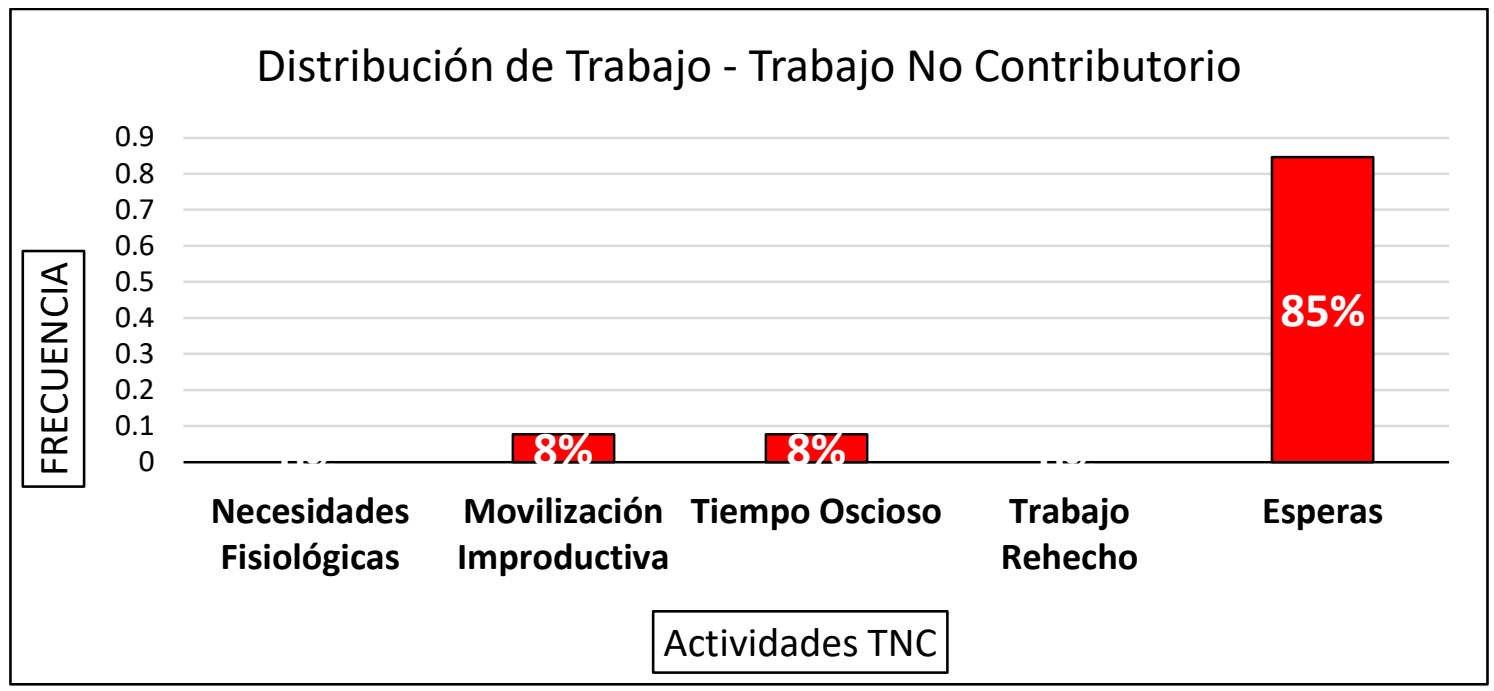

Fuente: Elaboración Propia. 
Tabla No 14: Carta Balance ${ }^{\circ} 2$ - Asentado de Ladrillo

\begin{tabular}{|c|c|c|c|c|c|c|c|c|c|c|c|}
\hline$N^{0}$ & Obrero\#1 & Obrero\#2 & Obrero\#3 & Obrero\#4 & Obrero\#5 & 46 & $E$ & NA & $C A$ & $\mathrm{CL}$ & $\mathrm{CL}$ \\
\hline 1 & NA & $\mathrm{AL}$ & $\mathrm{AL}$ & $\mathrm{TH}$ & $\mathrm{CL}$ & 47 & NA & NA & $C A$ & 1 & 1 \\
\hline 2 & $\mathrm{MI}$ & NA & NA & $\mathrm{TH}$ & $\mathrm{TH}$ & 48 & $\mathrm{AL}$ & NA & NA & $\mathrm{TH}$ & $\mathrm{TH}$ \\
\hline 3 & NA & NA & $\mathrm{NA}$ & $\mathrm{TH}$ & $\mathrm{ML}$ & 49 & $\mathrm{NA}$ & NA & $\mathrm{CM}$ & $\mathrm{TH}$ & $\mathrm{CL}$ \\
\hline 4 & $E$ & NA & $\mathrm{CA}$ & $\mathrm{TH}$ & $\mathrm{ML}$ & 50 & $\mathrm{CM}$ & NA & NA & TH & $\mathrm{CL}$ \\
\hline 5 & PM & PM & $L$ & 1 & $\mathrm{CL}$ & 51 & $\mathrm{CM}$ & PM & NA & $\mathrm{TH}$ & $\mathrm{TH}$ \\
\hline 6 & $C A$ & $C A$ & $C A$ & $\mathrm{TH}$ & $\mathrm{CL}$ & 52 & $\mathrm{AL}$ & NA & NA & $\mathrm{TH}$ & $\mathrm{CL}$ \\
\hline 7 & $\mathrm{CH}$ & $C A$ & $\mathrm{CA}$ & TO & $\mathrm{ML}$ & 53 & $\mathrm{NA}$ & 1 & $\mathrm{AL}$ & $\mathrm{TH}$ & $\mathrm{TH}$ \\
\hline 8 & $\mathrm{CM}$ & $C A$ & $L$ & TO & $\mathrm{TH}$ & 54 & $\mathrm{NA}$ & NA & $\mathrm{AL}$ & $\mathrm{TH}$ & $\mathrm{CL}$ \\
\hline 9 & PM & $C A$ & $\mathrm{AV}$ & TO & $\mathrm{CL}$ & 55 & $\mathrm{AL}$ & NA & NA & $\mathrm{TH}$ & $\mathrm{TH}$ \\
\hline 10 & $\mathrm{CM}$ & $\mathrm{CM}$ & $\mathrm{CH}$ & TO & $\mathrm{TH}$ & 56 & $\mathrm{NA}$ & NA & $\mathrm{CA}$ & $\mathrm{TH}$ & $\mathrm{TH}$ \\
\hline 11 & $\mathrm{CM}$ & $\mathrm{CM}$ & $\mathrm{CH}$ & TO & $\mathrm{ML}$ & 57 & NA & $C A$ & $C A$ & $\mathrm{~L}$ & $\mathrm{CL}$ \\
\hline 12 & NA & NA & NA & TO & $\mathrm{CL}$ & 58 & $\mathrm{CM}$ & PM & PM & $\mathrm{L}$ & $\mathrm{CL}$ \\
\hline 13 & NA & NA & PM & TO & $\mathrm{CL}$ & 59 & $\mathrm{CM}$ & $\mathrm{AL}$ & PM & $\mathrm{L}$ & $\mathrm{CL}$ \\
\hline 14 & $\mathrm{AL}$ & $\mathrm{AL}$ & $\mathrm{AL}$ & TO & $\mathrm{TH}$ & 60 & $\mathrm{CM}$ & $\mathrm{AL}$ & $\mathrm{CA}$ & $\mathrm{L}$ & $\mathrm{CL}$ \\
\hline 15 & $\mathrm{AL}$ & $\mathrm{AL}$ & $\mathrm{AL}$ & TO & $\mathrm{CL}$ & 61 & $\mathrm{~L}$ & $\mathrm{AL}$ & CA & $\mathrm{L}$ & $\mathrm{CL}$ \\
\hline 16 & NA & $\mathrm{AL}$ & $\mathrm{AL}$ & TO & $\mathrm{CL}$ & 62 & $\mathrm{~L}$ & $L$ & NA & $\mathrm{L}$ & $\mathrm{TH}$ \\
\hline 17 & $\mathrm{CA}$ & NA & $\mathrm{AL}$ & TO & $\mathrm{Cl}$ & 63 & $L$ & $\mathrm{CM}$ & NA & $\mathrm{TH}$ & $\mathrm{CL}$ \\
\hline 18 & $C A$ & NA & NA & TO & $\mathrm{CL}$ & 64 & $\mathrm{~L}$ & $\mathrm{AL}$ & $\mathrm{AL}$ & $\mathrm{L}$ & $\mathrm{CL}$ \\
\hline 19 & $\mathrm{NA}$ & $\mathrm{NA}$ & $\mathrm{CA}$ & TO & $\mathrm{CL}$ & 65 & $L$ & NA & $\mathrm{AL}$ & $L$ & $\mathrm{ML}$ \\
\hline 20 & NA & PM & L & TO & TO & 66 & L & $\mathrm{CM}$ & NA & L & $\mathrm{CL}$ \\
\hline 21 & NA & $C A$ & TO & TO & TO & 67 & $L$ & $\mathrm{AL}$ & $\mathrm{CM}$ & $\mathrm{L}$ & $\mathrm{CL}$ \\
\hline 22 & $\mathrm{CA}$ & TO & TO & TO & TO & 68 & PM & $\mathrm{AL}$ & $\mathrm{AL}$ & $L$ & $\mathrm{CL}$ \\
\hline 23 & TO & TO & TO & TO & TO & 69 & MI & NA & $\mathrm{AL}$ & $\mathrm{L}$ & $\mathrm{CL}$ \\
\hline 24 & TO & TO & TO & TO & TO & 70 & $\mathrm{AL}$ & $\mathrm{AL}$ & NA & TH & $\mathrm{CL}$ \\
\hline 25 & TO & TO & TO & TO & TO & 71 & $L$ & NA & NA & $\mathrm{TH}$ & $\mathrm{CL}$ \\
\hline 26 & TO & TO & TO & TO & TO & 72 & $E$ & PM & $\mathrm{CA}$ & TH & $\mathrm{ML}$ \\
\hline 27 & TO & TO & PM & $\mathrm{TH}$ & TO & 73 & $\mathrm{NA}$ & $\mathrm{PM}$ & $C A$ & $\mathrm{TH}$ & $\mathrm{ML}$ \\
\hline 28 & $\mathrm{NA}$ & TO & $\mathrm{CM}$ & $\mathrm{CL}$ & TO & 74 & $\mathrm{NA}$ & $C A$ & $\mathrm{CA}$ & $C A$ & $\mathrm{CL}$ \\
\hline 29 & PM & TO & $\mathrm{AL}$ & $\mathrm{CL}$ & TO & 75 & $\mathrm{NA}$ & $C A$ & $\mathrm{CA}$ & $\mathrm{CA}$ & $\mathrm{CL}$ \\
\hline 30 & $\mathrm{AL}$ & TH & $\mathrm{AL}$ & TH & $\mathrm{ML}$ & 76 & $\mathrm{~L}$ & $C A$ & PM & $\mathrm{E}$ & $\mathrm{CL}$ \\
\hline 31 & $\mathrm{AL}$ & $E$ & $\mathrm{AL}$ & $L$ & $\mathrm{ML}$ & 77 & $L$ & $\mathrm{CM}$ & $\mathrm{CA}$ & $E$ & $\mathrm{CL}$ \\
\hline 32 & $\mathrm{AL}$ & $\mathrm{CA}$ & $\mathrm{AL}$ & $L$ & $\mathrm{Cl}$ & 78 & $E$ & $\mathrm{CM}$ & $\mathrm{CH}$ & $\mathrm{TH}$ & $\mathrm{CL}$ \\
\hline 33 & NA & $\mathrm{CH}$ & $\mathrm{AL}$ & $L$ & $\mathrm{CL}$ & 79 & $\mathrm{~L}$ & $L$ & NA & TH & $\mathrm{CL}$ \\
\hline 34 & $C A$ & $\mathrm{CM}$ & NA & $\mathrm{TH}$ & $\mathrm{CL}$ & 80 & $\mathrm{TH}$ & MI & NA & $\mathrm{TH}$ & 1 \\
\hline 35 & $\mathrm{CA}$ & PM & $C A$ & $E$ & $\mathrm{CL}$ & 81 & TH & I & 1 & TH & $\mathrm{CL}$ \\
\hline 36 & 1 & NA & $C A$ & $\mathrm{TH}$ & $\mathrm{ML}$ & 82 & $\mathrm{NA}$ & 1 & 1 & $\mathrm{TH}$ & $\mathrm{CL}$ \\
\hline 37 & $C A$ & $\mathrm{CM}$ & $\mathrm{CM}$ & $\mathrm{TH}$ & $\mathrm{CL}$ & 83 & $E$ & $L$ & NA & $\mathrm{TH}$ & $\mathrm{ML}$ \\
\hline 38 & $\mathrm{~L}$ & $\mathrm{CM}$ & $\mathrm{AL}$ & $\mathrm{CL}$ & $\mathrm{CL}$ & 84 & $E$ & $L$ & TR & TH & $E$ \\
\hline 39 & $\mathrm{CH}$ & NA & $\mathrm{AL}$ & $\mathrm{CL}$ & $\mathrm{TH}$ & 85 & $\mathrm{TH}$ & $L$ & TR & $\mathrm{TH}$ & $E$ \\
\hline 40 & NA & $\mathrm{AL}$ & $\mathrm{AL}$ & $\mathrm{CL}$ & $\mathrm{TH}$ & 86 & MI & $L$ & $L$ & $\mathrm{TH}$ & $E$ \\
\hline 41 & NA & $\mathrm{AL}$ & NA & $\mathrm{CL}$ & $\mathrm{CL}$ & 87 & 1 & $L$ & $\mathrm{CH}$ & $\mathrm{E}$ & $E$ \\
\hline 42 & PM & $\mathrm{AL}$ & NA & $\mathrm{CL}$ & $\mathrm{CL}$ & 88 & $E$ & $E$ & $\mathrm{AL}$ & $\mathrm{TH}$ & $E$ \\
\hline 43 & $\mathrm{CM}$ & $\mathrm{NA}$ & PM & $\mathrm{CL}$ & $\mathrm{TH}$ & 89 & $E$ & $E$ & $\mathrm{AL}$ & $\mathrm{TH}$ & $E$ \\
\hline 44 & NA & NA & $\mathrm{CA}$ & $\mathrm{TH}$ & $\mathrm{CL}$ & 90 & $E$ & $E$ & $\mathrm{AL}$ & $\mathrm{TH}$ & $E$ \\
\hline 45 & NA & NA & $C A$ & $\mathrm{CL}$ & $\mathrm{CL}$ & & & & & & \\
\hline
\end{tabular}

Fuente: Elaboración Propia. 
Figura No 43: Distribución de Trabajo de Asentado de Ladrillo - Carta Balance ${ }^{\circ} 2$

\section{Distribución de Trabajo - Asentado de Ladrillo Blanco}

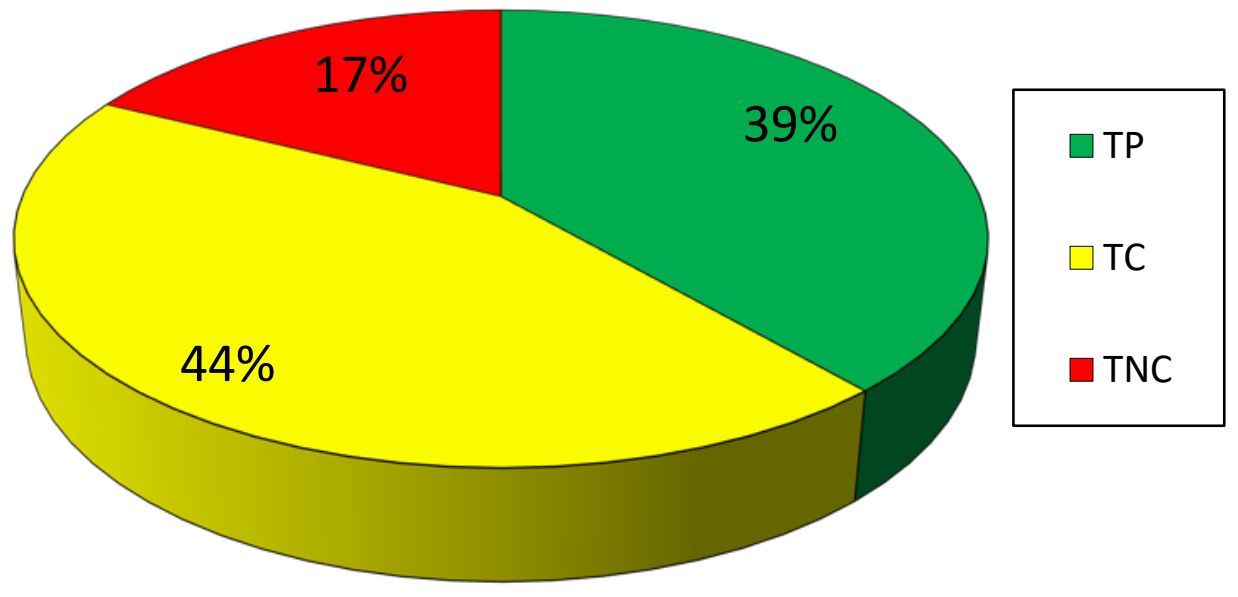

Fuente: Elaboración Propia.

Figura $N^{\circ}$ 44: Distribución de Trabajo Productivo - Carta Balance ${ }^{\circ} 2$

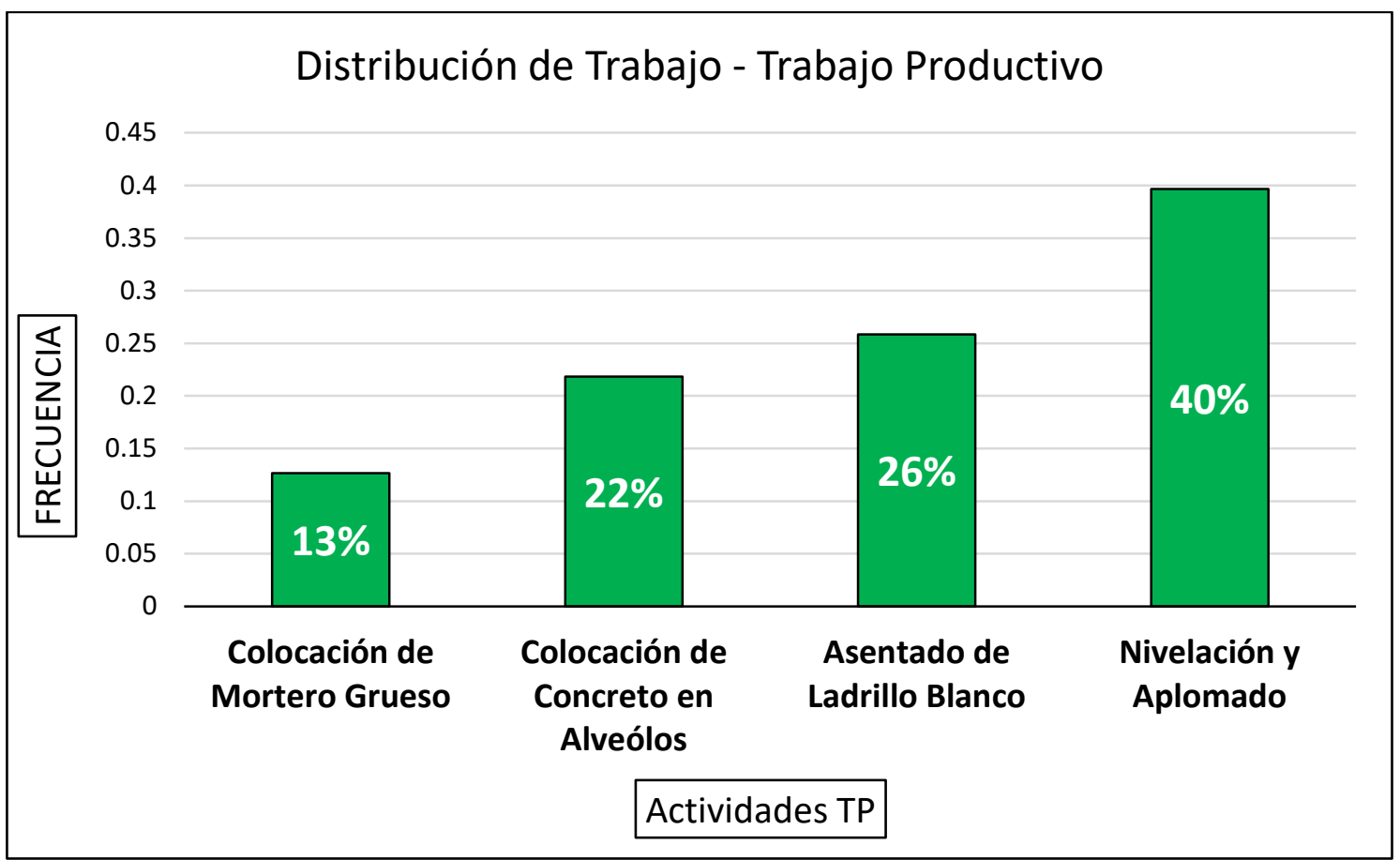

Fuente: Elaboración Propia. 
Figura $N^{\circ}$ 45: Distribución de Trabajo Contributorio - Carta Balance ${ }^{\circ} 2$

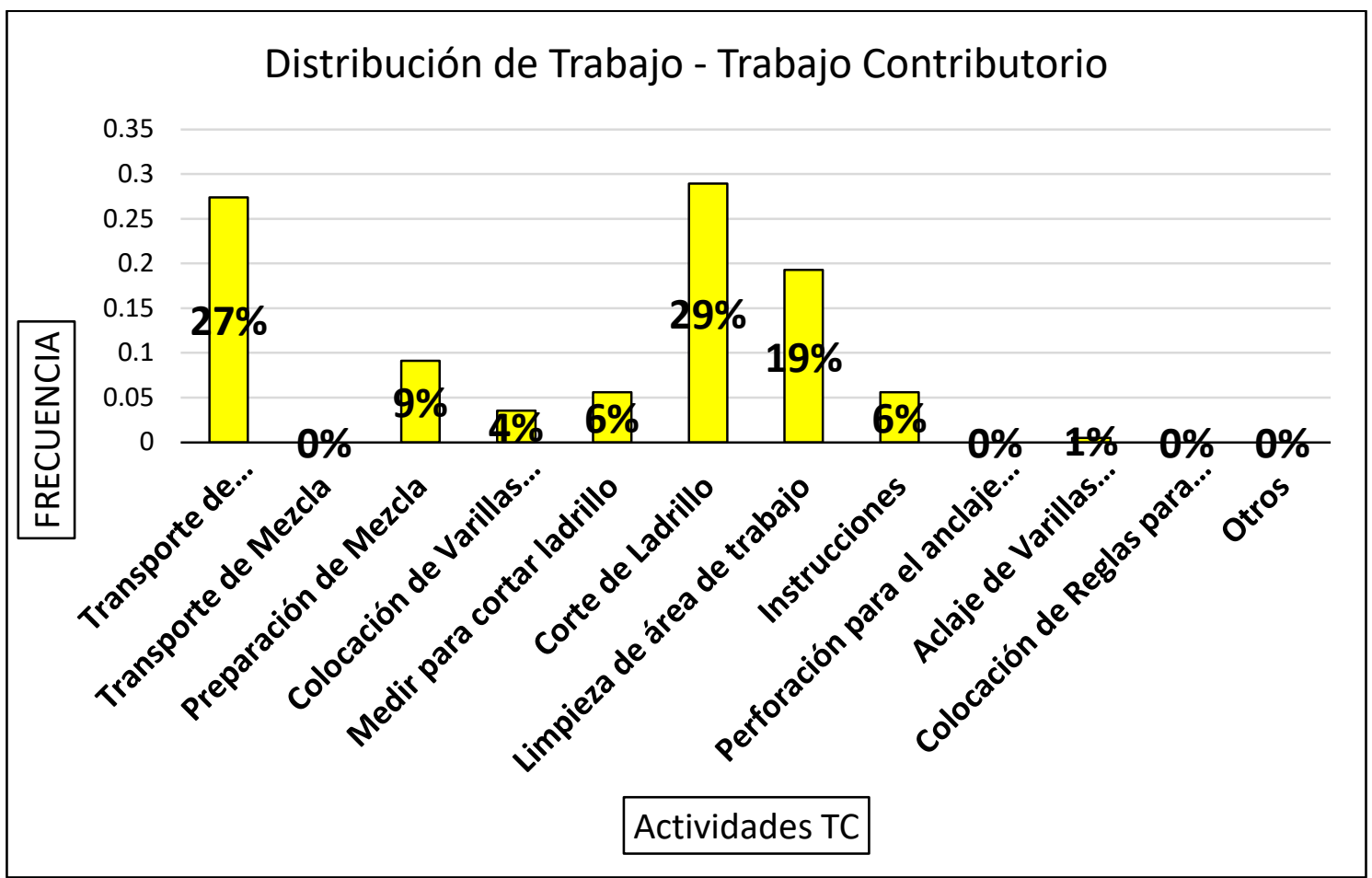

Fuente: Elaboración Propia.

Figura No 46: Distribución de Trabajo No Contributorio - Carta Balance ${ }^{\circ} 2$

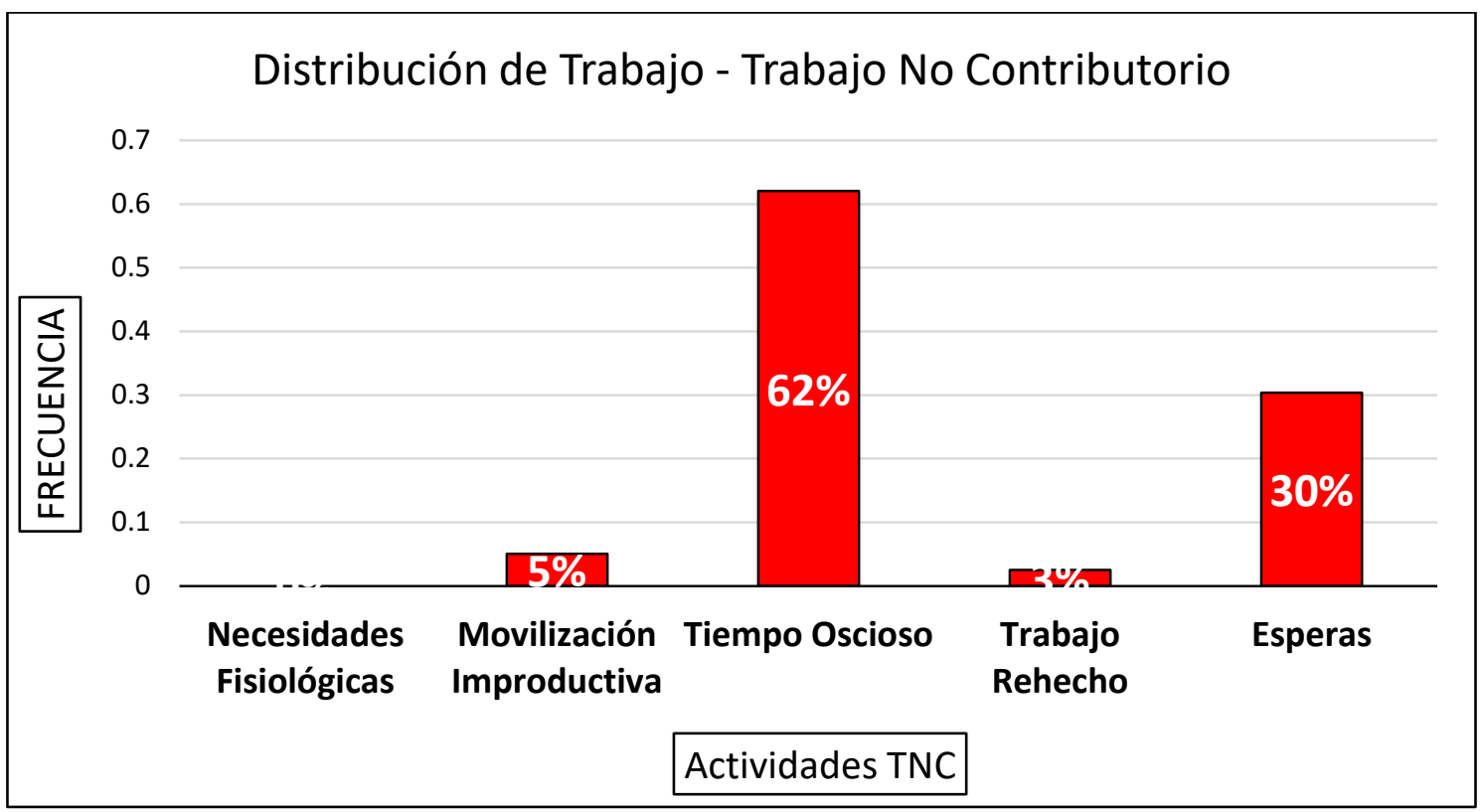

Fuente: Elaboración Propia. 
Tabla Nº 15: Carta Balance ${ }^{\circ} 3$ - Asentado de Ladrillo

\begin{tabular}{|c|c|c|c|c|c|c|c|}
\hline $\mathbf{N}^{0}$ & Obrero \#1 & Obrero \#2 & Obrero \#3 & 46 & $\mathrm{CA}$ & $\mathrm{TH}$ & $E$ \\
\hline 1 & CR & $\mathrm{TH}$ & $E$ & 47 & $\mathrm{CM}$ & $\mathrm{L}$ & $\mathrm{E}$ \\
\hline 2 & CR & $\mathrm{TH}$ & $E$ & 48 & $\mathrm{AL}$ & $\mathrm{L}$ & $\mathrm{E}$ \\
\hline 3 & $I$ & 1 & $E$ & 49 & $\mathrm{TH}$ & $\mathrm{L}$ & $\mathrm{E}$ \\
\hline 4 & $\mathrm{TH}$ & $\mathrm{TH}$ & $E$ & 50 & $\mathrm{TH}$ & $\mathrm{CR}$ & $\mathrm{E}$ \\
\hline 5 & $\mathrm{CR}$ & $\mathrm{TH}$ & $\mathrm{TH}$ & 51 & $\mathrm{CA}$ & $\mathrm{CR}$ & $\bar{E}$ \\
\hline 6 & CR & $\mathrm{TH}$ & $E$ & 52 & $\mathrm{CA}$ & $\mathrm{CR}$ & $E$ \\
\hline 7 & CR & $\mathrm{TH}$ & $E$ & 53 & $\mathrm{CM}$ & $\mathrm{CR}$ & $E$ \\
\hline 8 & $C R$ & $E$ & $E$ & 54 & $\mathrm{AL}$ & $\mathrm{CR}$ & $E$ \\
\hline 9 & $\mathrm{TH}$ & $\mathrm{E}$ & $E$ & 55 & NA & $\mathrm{CR}$ & $\mathrm{E}$ \\
\hline 10 & $E$ & $E$ & $E$ & 56 & $\mathrm{CA}$ & $\mathrm{CR}$ & $E$ \\
\hline 11 & $E$ & $E$ & $E$ & 57 & $\mathrm{CM}$ & $\mathrm{CR}$ & $\mathrm{E}$ \\
\hline 12 & $\mathrm{CR}$ & $\mathrm{E}$ & $E$ & 58 & $\mathrm{TH}$ & $\mathrm{CR}$ & $\mathrm{E}$ \\
\hline 13 & $\mathrm{CR}$ & $\mathrm{L}$ & $E$ & 59 & $\mathrm{AL}$ & $\mathrm{CR}$ & $E$ \\
\hline 14 & CR & $\mathrm{L}$ & $E$ & 60 & $\mathrm{CA}$ & CR & $E$ \\
\hline 15 & CR & $\mathrm{TH}$ & $E$ & 61 & $E$ & L & $E$ \\
\hline 16 & CR & $\mathrm{TH}$ & $E$ & 62 & $E$ & $\mathrm{~L}$ & $\mathrm{E}$ \\
\hline 17 & CR & PM & $E$ & 63 & $\mathrm{CL}$ & $\mathrm{L}$ & $\mathrm{E}$ \\
\hline 18 & CR & $E$ & $E$ & 64 & $\mathrm{AL}$ & $\mathrm{CR}$ & $\mathrm{E}$ \\
\hline 19 & CR & $\mathrm{AL}$ & $E$ & 65 & $\mathrm{AL}$ & $\mathrm{CR}$ & $\mathrm{E}$ \\
\hline 20 & CR & NA & $E$ & 66 & AV & CR & $E$ \\
\hline 21 & CR & NA & $E$ & 67 & $\mathrm{TH}$ & $\mathrm{CR}$ & $\mathrm{E}$ \\
\hline 22 & 1 & $C A$ & $E$ & 68 & $\mathrm{CA}$ & $\mathrm{CR}$ & $\mathrm{E}$ \\
\hline 23 & 1 & PM & $E$ & 69 & $\mathrm{TH}$ & NA & $E$ \\
\hline 24 & $C R$ & 1 & $E$ & 70 & $\mathrm{AL}$ & $\mathrm{ML}$ & $E$ \\
\hline 25 & CR & $\mathrm{AL}$ & $E$ & 71 & $\mathrm{AL}$ & $\mathrm{ML}$ & $E$ \\
\hline 26 & TO & $\mathrm{AL}$ & $E$ & 72 & $\mathrm{CA}$ & $\mathrm{ML}$ & $\mathrm{E}$ \\
\hline 27 & $C R$ & $\mathrm{PM}$ & $E$ & 73 & $\mathrm{CA}$ & $\mathrm{ML}$ & $E$ \\
\hline 28 & 1 & $\mathrm{CA}$ & $E$ & 74 & $\mathrm{TH}$ & $\mathrm{ML}$ & $E$ \\
\hline 29 & TO & $\mathrm{CM}$ & $E$ & 75 & $\mathrm{AL}$ & $\mathrm{CL}$ & $E$ \\
\hline 30 & $\mathrm{CR}$ & $\mathrm{AL}$ & $E$ & 76 & $\mathrm{CA}$ & $\mathrm{ML}$ & $E$ \\
\hline 31 & $\mathrm{CR}$ & $\mathrm{AL}$ & $E$ & 77 & $\mathrm{CA}$ & $\mathrm{ML}$ & $E$ \\
\hline 32 & PM & $\mathrm{AL}$ & $E$ & 78 & $\mathrm{CA}$ & $\mathrm{TH}$ & $E$ \\
\hline 33 & MI & $\mathrm{PM}$ & $E$ & 79 & $\mathrm{PM}$ & PM & $E$ \\
\hline 34 & $\mathrm{CL}$ & $L$ & $E$ & 80 & $E$ & $\mathrm{PM}$ & $E$ \\
\hline 35 & $\mathrm{CL}$ & $L$ & $E$ & 81 & $\mathrm{PM}$ & $\mathrm{CM}$ & $E$ \\
\hline 36 & $\mathrm{TH}$ & $L$ & $E$ & 82 & $\mathrm{AL}$ & $\mathrm{AL}$ & $E$ \\
\hline 37 & TO & $L$ & $E$ & 83 & TO & TO & $E$ \\
\hline 38 & TO & $L$ & $E$ & 84 & TO & TO & $E$ \\
\hline 39 & TO & TO & $E$ & 85 & NA & TO & $E$ \\
\hline 40 & TO & TO & $E$ & 86 & TO & TO & $E$ \\
\hline 41 & $\mathrm{AL}$ & $L$ & $E$ & 87 & TO & TO & $E$ \\
\hline 42 & NA & $\mathrm{TH}$ & $E$ & 88 & TO & TO & $E$ \\
\hline 43 & $\mathrm{TH}$ & $\mathrm{TH}$ & $E$ & 89 & TO & TO & $E$ \\
\hline 44 & NA & $\mathrm{TH}$ & $E$ & 90 & TO & TO & $E$ \\
\hline 45 & $\mathrm{CA}$ & $\mathrm{TH}$ & $E$ & & & & \\
\hline
\end{tabular}

Fuente: Elaboración Propia. 
Figura No 47: Distribución de Trabajo de Asentado de Ladrillo - Carta Balance ${ }^{\circ} 3$

Distribución de Trabajo - Asentado de Ladrillo Blanco

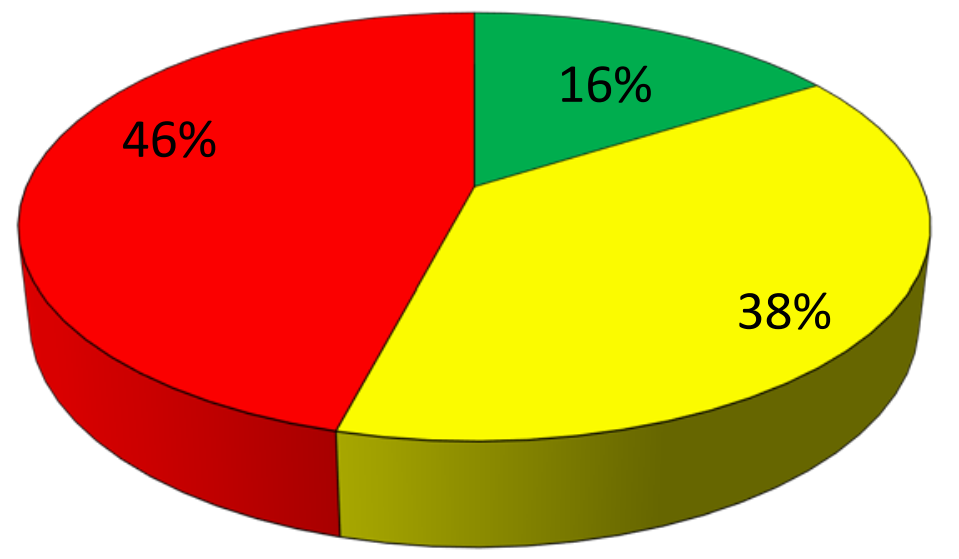

TNC

Fuente: Elaboración Propia.

Figura Nº 48: Distribución de Trabajo Productivo - Carta Balance ${ }^{\circ} 3$

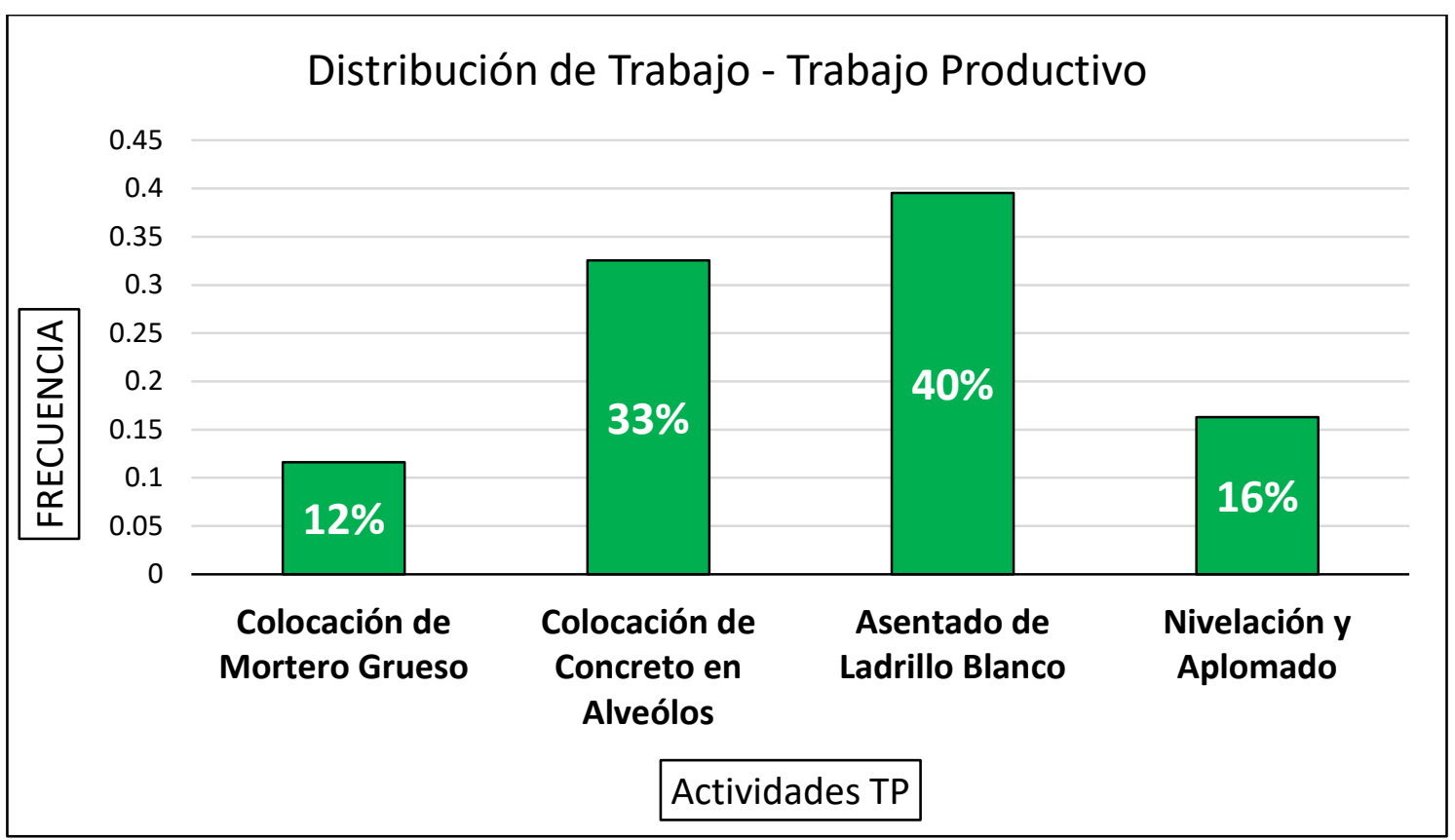

Fuente: Elaboración Propia. 
Figura Nº 49: Distribución de Trabajo Contributorio - Carta Balance ${ }^{\circ} 3$

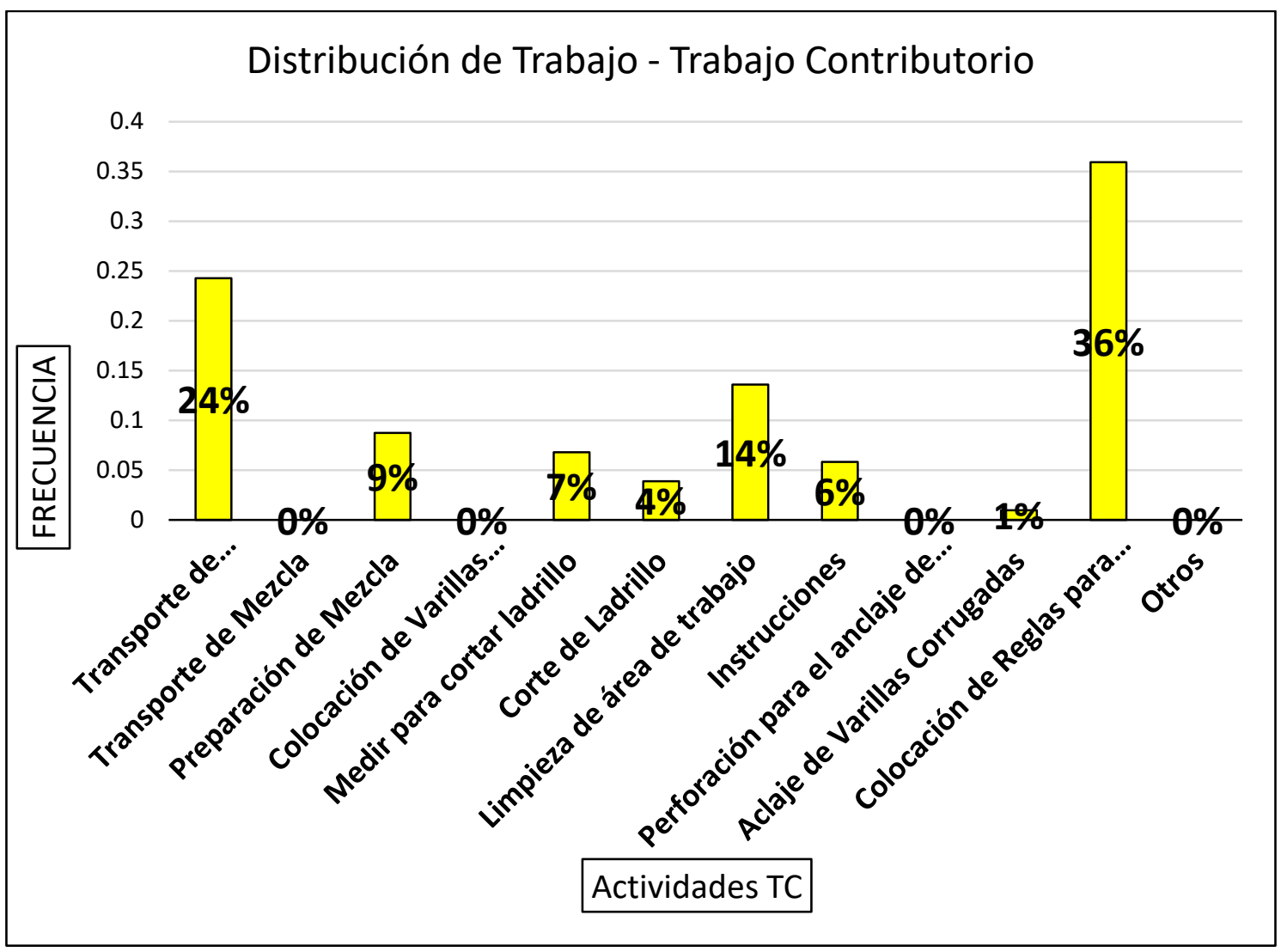

Fuente: Elaboración Propia.

Figura $N^{\circ}$ 50: Distribución de Trabajo No Contributorio - Carta Balance ${ }^{\circ} 3$

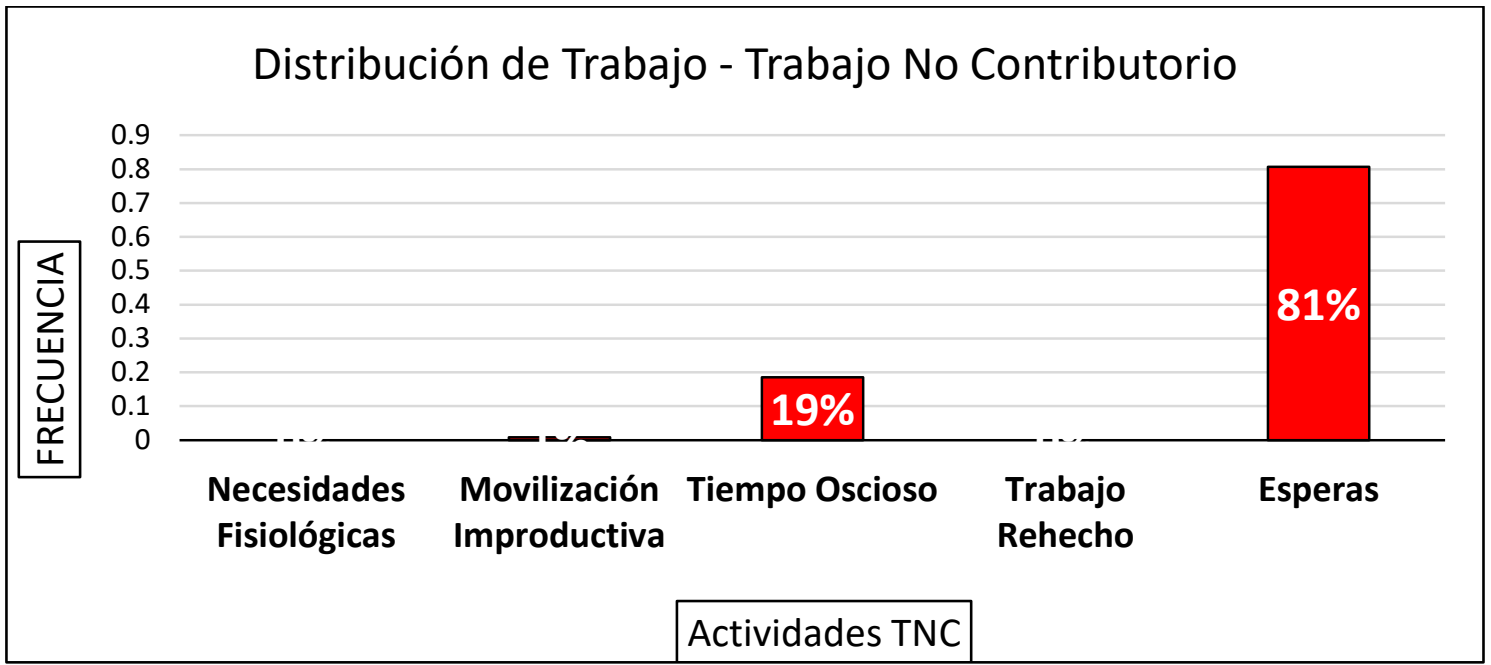

Fuente: Elaboración Propia. 
Tabla N ${ }^{\circ}$ 16: Carta Balance ${ }^{\circ} 4$ - Asentado de Ladrillo

\begin{tabular}{|c|c|c|c|c|c|c|c|c|c|}
\hline $\mathrm{N}^{0}$ & Obrero \#1 & Obrero \#2 & Obrero \#3 & Obrero \#4 & 46 & NA & NA & $\mathrm{AL}$ & $\mathrm{TH}$ \\
\hline 1 & TO & $\mathrm{AL}$ & $\mathrm{AL}$ & $\mathrm{CL}$ & 47 & $\mathrm{CH}$ & NA & $\mathrm{AL}$ & $\mathrm{CL}$ \\
\hline 2 & MI & $\mathrm{CL}$ & $\mathrm{CL}$ & $\mathrm{CL}$ & 48 & NA & $\mathrm{CA}$ & NA & $\mathrm{TH}$ \\
\hline 3 & $\mathrm{TH}$ & NA & $\mathrm{NA}$ & $\mathrm{CL}$ & 49 & $\mathrm{CM}$ & $\mathrm{CA}$ & TM & $M L$ \\
\hline 4 & PM & $\mathrm{TH}$ & $\mathrm{TH}$ & $\mathrm{CL}$ & 50 & PM & $\mathrm{TH}$ & PM & $\mathrm{CL}$ \\
\hline 5 & $\mathrm{CA}$ & $\mathrm{TH}$ & $\mathrm{CA}$ & $\mathrm{CL}$ & 51 & $\mathrm{PM}$ & $\mathrm{TH}$ & NA & $\mathrm{CL}$ \\
\hline 6 & $\mathrm{~L}$ & $\mathrm{TH}$ & NA & $\mathrm{CL}$ & 52 & $\mathrm{AL}$ & $\mathrm{AL}$ & $\mathrm{CA}$ & $\mathrm{CL}$ \\
\hline 7 & $L$ & PM & $\mathrm{CH}$ & $\mathrm{CL}$ & 53 & $\mathrm{AL}$ & $\mathrm{AL}$ & $\mathrm{CA}$ & $\mathrm{TH}$ \\
\hline 8 & $\mathrm{~L}$ & $\mathrm{ML}$ & TO & TO & 54 & $\mathrm{CA}$ & $\mathrm{CA}$ & NA & $\mathrm{ML}$ \\
\hline 9 & NA & ML & TO & $\mathrm{CL}$ & 55 & PM & PM & NA & $\mathrm{ML}$ \\
\hline 10 & $\mathrm{PM}$ & $\mathrm{ML}$ & $\mathrm{ML}$ & $\mathrm{CL}$ & 56 & $\mathrm{CH}$ & $\mathrm{CH}$ & $\mathrm{PM}$ & $\mathrm{TH}$ \\
\hline 11 & NA & $\mathrm{AL}$ & $\mathrm{CM}$ & $\mathrm{CL}$ & 57 & PM & PM & $\mathrm{TH}$ & $\mathrm{TH}$ \\
\hline 12 & NA & NA & $\mathrm{AL}$ & $\mathrm{CL}$ & 58 & $\mathrm{CM}$ & $\mathrm{CM}$ & $\mathrm{CM}$ & $\mathrm{CL}$ \\
\hline 13 & $\mathrm{NA}$ & $\mathrm{ML}$ & $\mathrm{AL}$ & $\mathrm{CL}$ & 59 & $\mathrm{AL}$ & $\mathrm{AL}$ & $\mathrm{CM}$ & $\mathrm{CL}$ \\
\hline 14 & $\mathrm{CM}$ & NA & $\mathrm{NA}$ & $\mathrm{CL}$ & 60 & $\mathrm{ML}$ & NA & NA & $\mathrm{TH}$ \\
\hline 15 & $\mathrm{CM}$ & $\mathrm{ML}$ & AV & $\mathrm{ML}$ & 61 & $\mathrm{CL}$ & $\mathrm{AL}$ & $\mathrm{AL}$ & $\mathrm{TH}$ \\
\hline 16 & $\mathrm{AL}$ & $\mathrm{ML}$ & AV & $\mathrm{CL}$ & 62 & NA & $\mathrm{AL}$ & $\mathrm{AL}$ & $E$ \\
\hline 17 & $\mathrm{AL}$ & $\mathrm{AL}$ & NA & $\mathrm{CL}$ & 63 & $\mathrm{PM}$ & NA & $\mathrm{AL}$ & $\mathrm{ML}$ \\
\hline 18 & $\mathrm{AL}$ & NA & PM & $\mathrm{TH}$ & 64 & $\mathrm{CA}$ & NA & $\mathrm{AL}$ & $\mathrm{ML}$ \\
\hline 19 & TH & $\mathrm{CA}$ & $\mathrm{CA}$ & $\mathrm{ML}$ & 65 & $\mathrm{~L}$ & $\mathrm{~L}$ & $\mathrm{AL}$ & $\mathrm{CL}$ \\
\hline 20 & MI & TO & TO & $\mathrm{CL}$ & 66 & $\mathrm{CM}$ & $\mathrm{CA}$ & NA & $\mathrm{TH}$ \\
\hline 21 & $\mathrm{TH}$ & $\mathrm{CM}$ & $\mathrm{CM}$ & $\mathrm{TH}$ & 67 & $\mathrm{ML}$ & $\mathrm{CA}$ & $L$ & $\mathrm{TH}$ \\
\hline 22 & TH & $\mathrm{CL}$ & $\mathrm{AL}$ & $\mathrm{CL}$ & 68 & $\mathrm{CL}$ & $\mathrm{CH}$ & $\mathrm{MI}$ & $\mathrm{TH}$ \\
\hline 23 & TH & NA & $\mathrm{AL}$ & $\mathrm{CL}$ & 69 & $\mathrm{AL}$ & $\mathrm{AL}$ & $\mathrm{MI}$ & $\mathrm{TH}$ \\
\hline 24 & $\mathrm{TH}$ & $\mathrm{AL}$ & $\mathrm{AL}$ & $\mathrm{CL}$ & 70 & $\mathrm{AL}$ & NA & $\mathrm{MI}$ & $\mathrm{CL}$ \\
\hline 25 & $\mathrm{CA}$ & NA & $\mathrm{AL}$ & $\mathrm{TH}$ & 71 & $\mathrm{TM}$ & $\mathrm{AL}$ & $\mathrm{MI}$ & $\mathrm{CL}$ \\
\hline 26 & $\mathrm{AV}$ & $\mathrm{CA}$ & NA & $\mathrm{ML}$ & 72 & PM & NA & $L$ & $\mathrm{CL}$ \\
\hline 27 & $\mathrm{AV}$ & $\mathrm{AV}$ & $\mathrm{CA}$ & $\mathrm{TH}$ & 73 & $\mathrm{CA}$ & $L$ & $L$ & $\mathrm{CL}$ \\
\hline 28 & $\mathrm{CH}$ & $\mathrm{CH}$ & $\mathrm{CA}$ & $\mathrm{CL}$ & 74 & $\mathrm{CA}$ & NA & $\mathrm{CA}$ & $\mathrm{CL}$ \\
\hline 29 & $\mathrm{CM}$ & $\mathrm{CM}$ & NA & $\mathrm{CL}$ & 75 & $\mathrm{ML}$ & $\mathrm{PM}$ & MI & $\mathrm{ML}$ \\
\hline 30 & $\mathrm{TH}$ & $\mathrm{AL}$ & $\mathrm{CH}$ & $\mathrm{CL}$ & 76 & $E$ & $\mathrm{CA}$ & PM & $\mathrm{ML}$ \\
\hline 31 & $\mathrm{AL}$ & $\mathrm{AL}$ & $\mathrm{AL}$ & $\mathrm{CL}$ & 77 & $E$ & $\mathrm{CA}$ & $\mathrm{CA}$ & $\mathrm{CL}$ \\
\hline 32 & NA & $\mathrm{ML}$ & $\mathrm{AL}$ & $\mathrm{ML}$ & 78 & $\mathrm{PM}$ & $\mathrm{L}$ & $L$ & $\mathrm{ML}$ \\
\hline 33 & $\mathrm{CA}$ & NA & $\mathrm{AL}$ & $\mathrm{CL}$ & 79 & $\mathrm{CM}$ & $L$ & $\mathrm{ML}$ & $\mathrm{ML}$ \\
\hline 34 & $L$ & NA & $\mathrm{AL}$ & $\mathrm{ML}$ & 80 & NA & $\mathrm{TM}$ & $\mathrm{MI}$ & $\mathrm{CL}$ \\
\hline 35 & $\mathrm{PM}$ & $\mathrm{PM}$ & NA & $\mathrm{TH}$ & 81 & $\mathrm{PM}$ & $\mathrm{CM}$ & $\mathrm{L}$ & $L$ \\
\hline 36 & $\mathrm{AL}$ & $\mathrm{CA}$ & $\mathrm{PM}$ & $\mathrm{CL}$ & 82 & $\mathrm{AL}$ & NA & $\mathrm{L}$ & $\mathrm{CL}$ \\
\hline 37 & MI & PM & $\mathrm{CA}$ & $\mathrm{ML}$ & 83 & TO & $\mathrm{AL}$ & $L$ & $\mathrm{CL}$ \\
\hline 38 & $\mathrm{TH}$ & $\mathrm{ML}$ & $\mathrm{CA}$ & $\mathrm{ML}$ & 84 & NA & $\mathrm{AL}$ & 1 & $\mathrm{CL}$ \\
\hline 39 & $\mathrm{TH}$ & $\mathrm{CM}$ & $L$ & $\mathrm{CL}$ & 85 & $\mathrm{PM}$ & NA & $L$ & $L$ \\
\hline 40 & $\mathrm{PM}$ & $\mathrm{ML}$ & $L$ & TH & 86 & $\mathrm{CA}$ & NA & $L$ & $\mathrm{~L}$ \\
\hline 41 & $\mathrm{CA}$ & $\mathrm{AL}$ & $\mathrm{PM}$ & $\mathrm{CL}$ & 87 & $\mathrm{CA}$ & $\mathrm{PM}$ & $L$ & $L$ \\
\hline 42 & $\mathrm{CM}$ & $\mathrm{AL}$ & $\mathrm{CM}$ & $\mathrm{TH}$ & 88 & $L$ & $\mathrm{CA}$ & $L$ & $L$ \\
\hline 43 & $\mathrm{AL}$ & $\mathrm{AL}$ & $\mathrm{NA}$ & $\mathrm{CL}$ & 89 & $\mathrm{~L}$ & $\mathrm{CA}$ & $\mathrm{L}$ & $\mathrm{L}$ \\
\hline 44 & $\mathrm{CH}$ & $\mathrm{AL}$ & $\mathrm{AL}$ & $\mathrm{CL}$ & 90 & $L$ & $\mathrm{CA}$ & $\mathrm{L}$ & $\mathrm{L}$ \\
\hline 45 & $\mathrm{CL}$ & $\mathrm{AV}$ & $\mathrm{AL}$ & $\mathrm{CL}$ & & & & & \\
\hline
\end{tabular}

Fuente: Elaboración Propia. 
Figura $N^{\circ}$ 51: Distribución de Trabajo de Asentado de Ladrillo - Carta Balance ${ }^{\circ} 4$

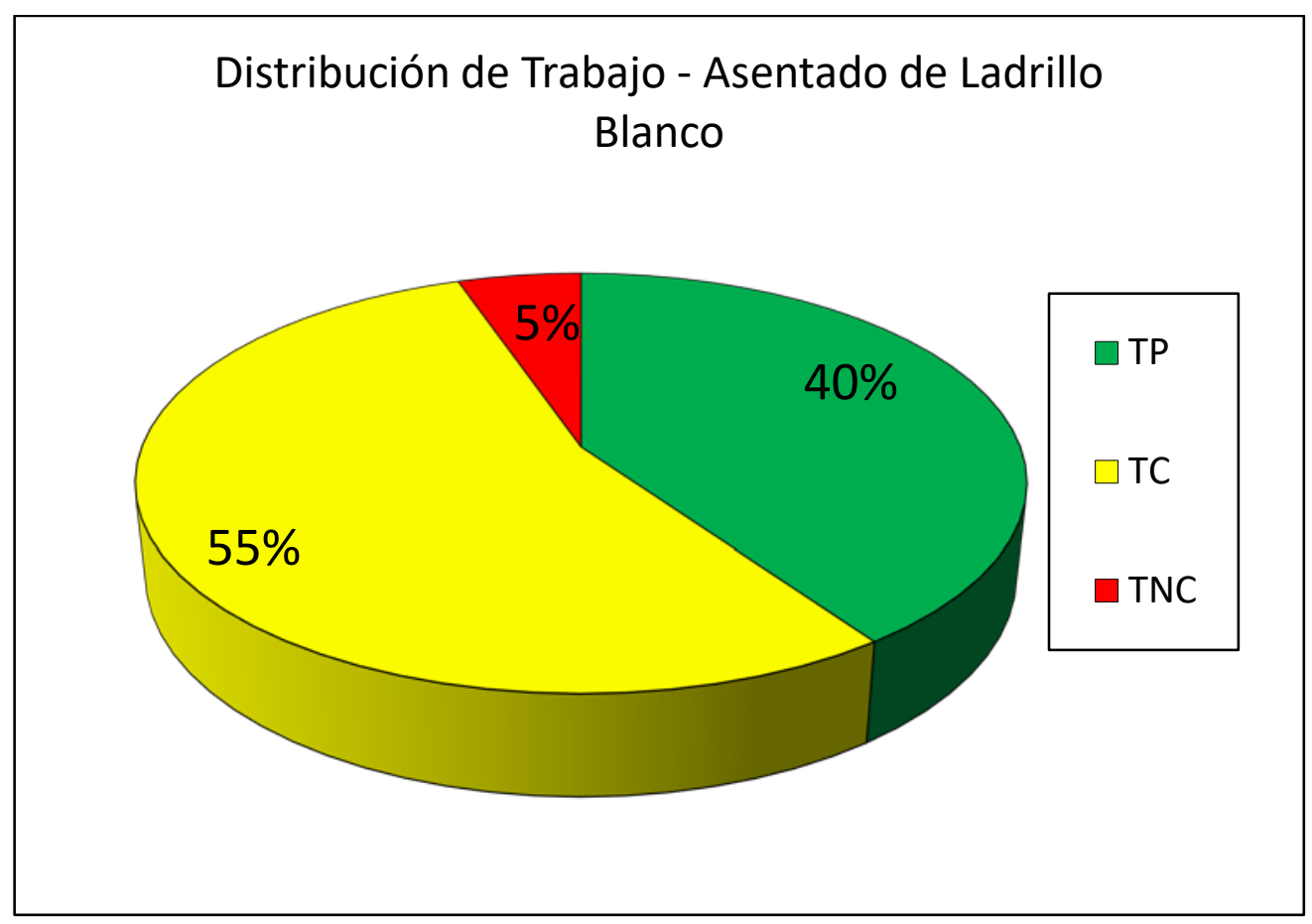

Fuente: Elaboración Propia.

Figura $N^{\circ}$ 52: Distribución de Trabajo Productivo - Carta Balance ${ }^{\circ} 4$

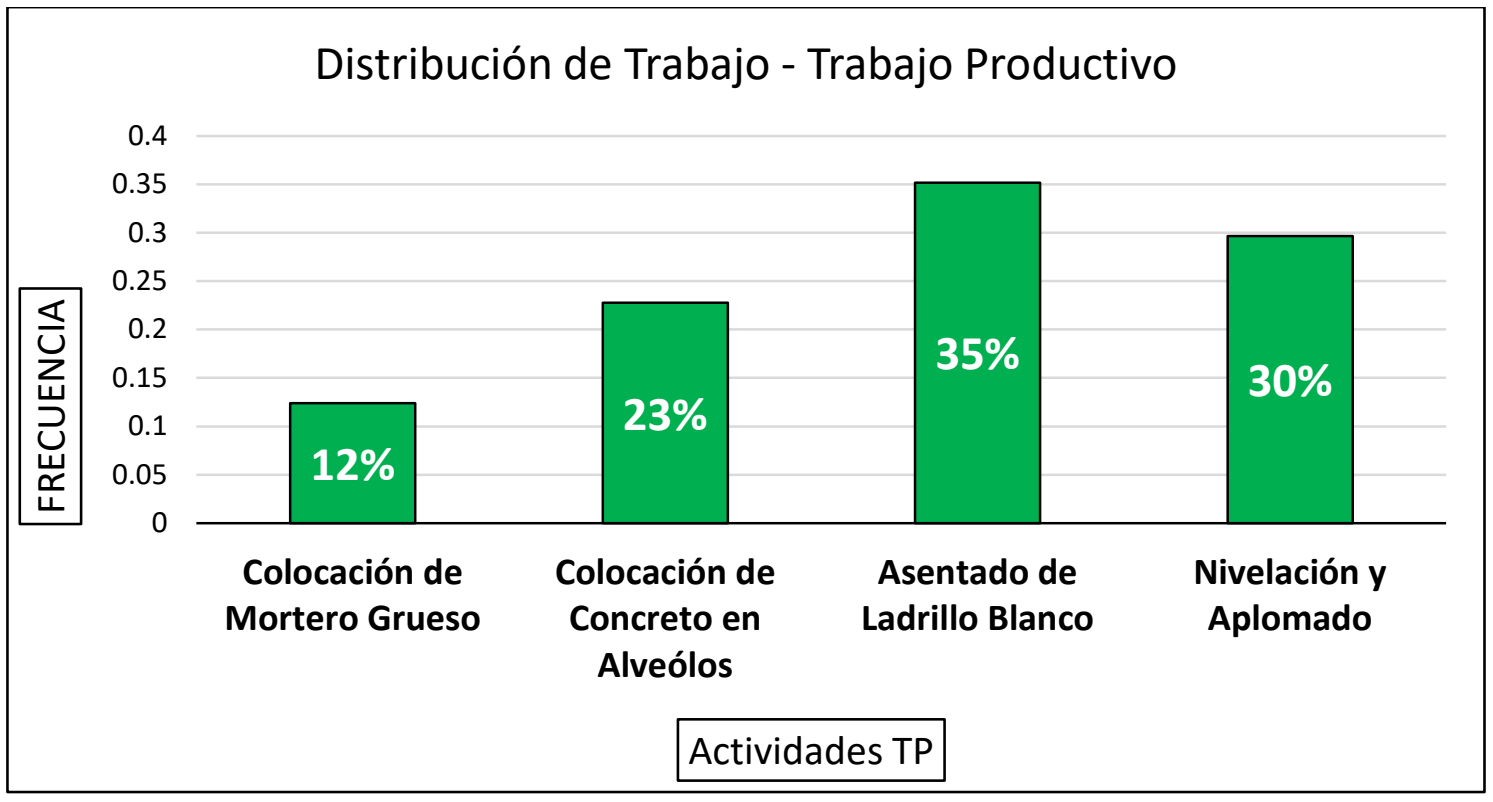

Fuente: Elaboración Propia. 
Figura N 53: Distribución de Trabajo Contributorio - Carta Balance ${ }^{\circ} 4$

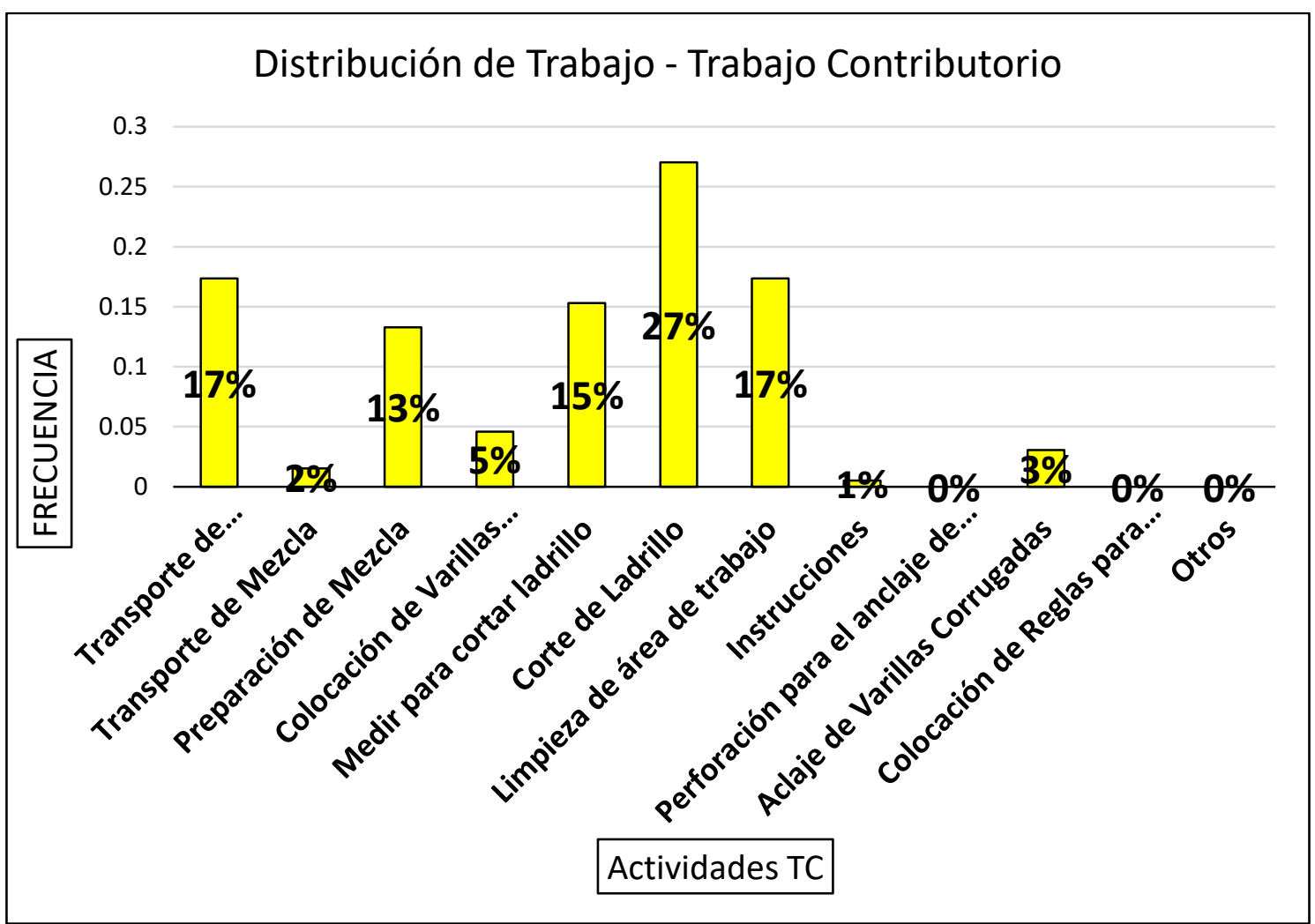

Fuente: Elaboración Propia.

Figura No 54: Distribución de Trabajo No Contributorio - Carta Balance ${ }^{\circ} 4$

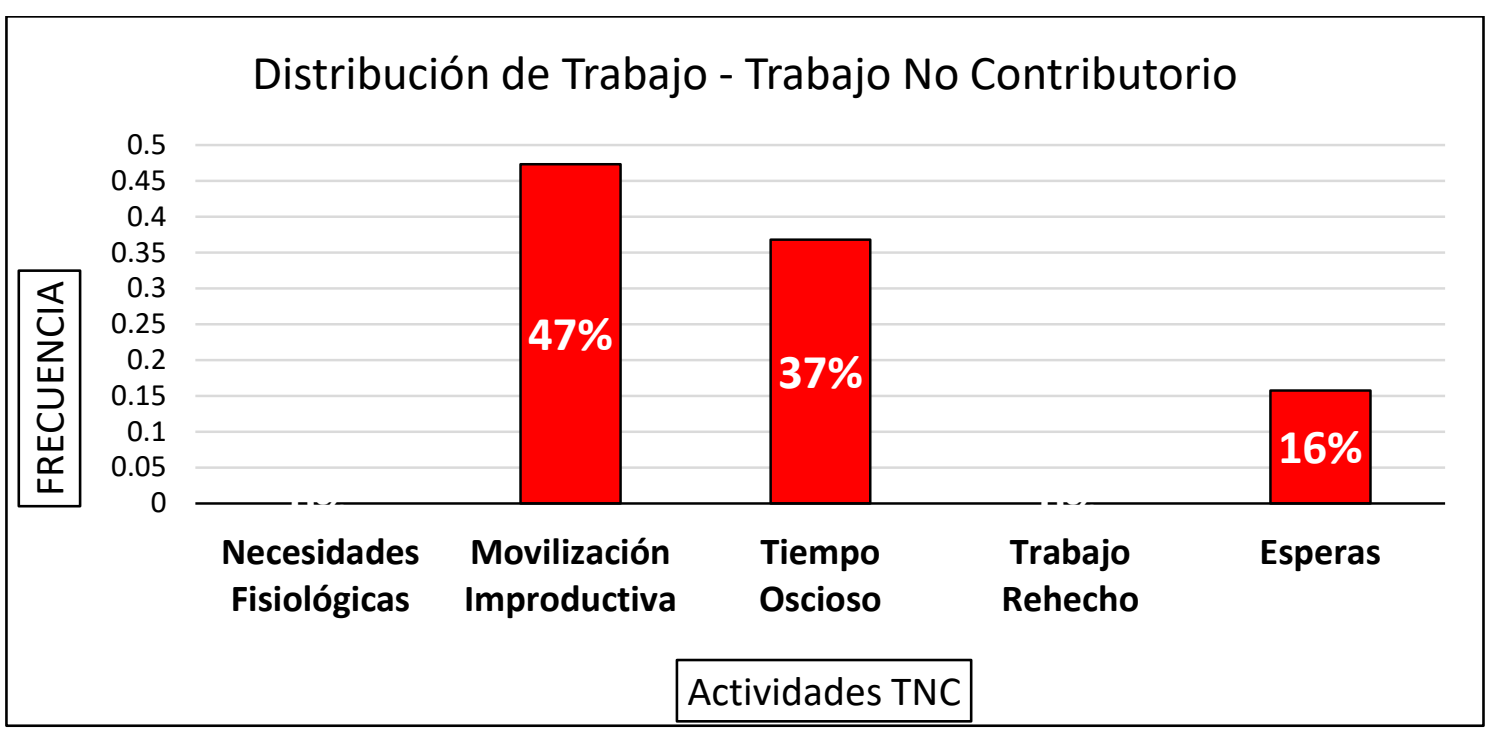

Fuente: Elaboración Propia. 
Tabla N 17: Carta Balance ${ }^{\circ} 5$ - Asentado de Ladrillo

\begin{tabular}{|c|c|c|c|c|c|c|c|c|c|c|c|}
\hline $\mathrm{N}^{0}$ & Obrero\#1 & Obrero\#2 & Obrero\#3 & Obrero \#4 & Obrero\#5 & 46 & $\mathrm{CM}$ & $C A$ & $\mathrm{AL}$ & $\mathrm{CL}$ & $\mathrm{TH}$ \\
\hline 1 & $C R$ & 1 & 1 & $E$ & $\mathrm{TH}$ & 47 & $\mathrm{AL}$ & $C A$ & $E$ & $\mathrm{CL}$ & $\mathrm{TH}$ \\
\hline 2 & $C R$ & 1 & 1 & $E$ & TH & 48 & $\mathrm{AL}$ & AV & NA & $\mathrm{CL}$ & $\mathrm{TH}$ \\
\hline 3 & $C R$ & 1 & 1 & $E$ & L & 49 & NA & PM & NA & $\mathrm{CL}$ & $\mathrm{CL}$ \\
\hline 4 & $C R$ & 1 & 1 & $E$ & $\mathrm{TM}$ & 50 & NA & NA & NA & $\mathrm{CL}$ & $\mathrm{CL}$ \\
\hline 5 & $C R$ & 1 & 1 & $E$ & TH & 51 & $C A$ & NA & NA & $\mathrm{CL}$ & $\mathrm{CL}$ \\
\hline 6 & $C R$ & $\mathrm{CH}$ & NA & $\mathrm{TH}$ & TH & 52 & $C A$ & $C A$ & PM & $\mathrm{CL}$ & $\mathrm{CL}$ \\
\hline 7 & $C R$ & $\mathrm{CH}$ & NA & $E$ & L & 53 & NA & $\mathrm{TH}$ & $\mathrm{CA}$ & $\mathrm{CL}$ & $\mathrm{TH}$ \\
\hline 8 & $C R$ & $\mathrm{CM}$ & NA & $E$ & L & 54 & $C A$ & PM & $C A$ & $\mathrm{CL}$ & $\mathrm{TH}$ \\
\hline 9 & $C R$ & $\mathrm{CM}$ & $\mathrm{L}$ & $\mathrm{ML}$ & $\mathrm{CL}$ & 55 & PM & $\mathrm{CM}$ & $\mathrm{CH}$ & ML & $\mathrm{TH}$ \\
\hline 10 & $C R$ & $\mathrm{CM}$ & $\mathrm{ML}$ & $\mathrm{ML}$ & $\mathrm{CL}$ & 56 & $\mathrm{CM}$ & $\mathrm{AL}$ & $\mathrm{CH}$ & ML & $\mathrm{ML}$ \\
\hline 11 & $C R$ & $\mathrm{AL}$ & $\mathrm{ML}$ & $\mathrm{ML}$ & $\mathrm{TH}$ & 57 & $\mathrm{AL}$ & $\mathrm{AL}$ & PM & ML & $M L$ \\
\hline 12 & $C R$ & $\mathrm{AL}$ & $\mathrm{ML}$ & $\mathrm{ML}$ & $\mathrm{ML}$ & 58 & NA & NA & $\mathrm{CM}$ & $\mathrm{CL}$ & $\mathrm{TH}$ \\
\hline 13 & $C R$ & $\mathrm{AL}$ & $\mathrm{ML}$ & $\mathrm{ML}$ & $\mathrm{TH}$ & 59 & $\mathrm{AL}$ & PM & $\mathrm{CM}$ & $\mathrm{CL}$ & $\mathrm{TH}$ \\
\hline 14 & $L$ & $\mathrm{AL}$ & $\mathrm{ML}$ & $\mathrm{ML}$ & $\mathrm{CL}$ & 60 & PM & PM & PM & $\mathrm{CL}$ & $\mathrm{TH}$ \\
\hline 15 & PM & $\mathrm{TH}$ & $\mathrm{ML}$ & $\mathrm{ML}$ & $\mathrm{CL}$ & 61 & $C A$ & $\mathrm{ML}$ & NA & $\mathrm{CL}$ & $\mathrm{TH}$ \\
\hline 16 & L & NA & $\mathrm{ML}$ & $\mathrm{TH}$ & $\mathrm{TH}$ & 62 & $\mathrm{CH}$ & NA & $\mathrm{AL}$ & $E$ & $\mathrm{TH}$ \\
\hline 17 & PM & NA & NA & $\mathrm{CL}$ & TH & 63 & $\mathrm{AL}$ & NA & $\mathrm{AL}$ & $\mathrm{ML}$ & $\mathrm{TH}$ \\
\hline 18 & NA & TH & PM & $\mathrm{CL}$ & TH & 64 & $\mathrm{AL}$ & NA & $\mathrm{AL}$ & $\mathrm{CL}$ & $\mathrm{TH}$ \\
\hline 19 & NA & NA & PM & $\mathrm{CL}$ & TH & 65 & $\mathrm{AL}$ & $C A$ & $\mathrm{AL}$ & $\mathrm{CL}$ & $\mathrm{TH}$ \\
\hline 20 & NA & $\mathrm{CA}$ & $\mathrm{CM}$ & $\mathrm{CL}$ & 1 & 66 & NA & PM & $\mathrm{AL}$ & $\mathrm{ML}$ & $\mathrm{TH}$ \\
\hline 21 & $E$ & PM & 1 & $\mathrm{CL}$ & $\mathrm{CL}$ & 67 & PM & PM & $\mathrm{AL}$ & ML & $\mathrm{TH}$ \\
\hline 22 & $E$ & NA & 1 & $\mathrm{CL}$ & TH & 68 & $\mathrm{CA}$ & $C A$ & $\mathrm{AL}$ & $\mathrm{ML}$ & $\mathrm{TH}$ \\
\hline 23 & MI & $\mathrm{CM}$ & 1 & $\mathrm{CL}$ & MI & 69 & $\mathrm{CM}$ & $\mathrm{CH}$ & $\mathrm{AL}$ & $\mathrm{CL}$ & $\mathrm{TH}$ \\
\hline 24 & $E$ & $\mathrm{CM}$ & $E$ & $\mathrm{CL}$ & MI & 70 & $\mathrm{AL}$ & $\mathrm{CH}$ & $\mathrm{ML}$ & $\mathrm{ML}$ & $\mathrm{TH}$ \\
\hline 25 & $\mathrm{ML}$ & $\mathrm{CM}$ & $\mathrm{CM}$ & $\mathrm{Cl}$ & MI & 71 & $\mathrm{AL}$ & PM & NA & $\mathrm{CL}$ & $\mathrm{TH}$ \\
\hline 26 & $\mathrm{ML}$ & AL & $\mathrm{CM}$ & TH & MI & 72 & $\mathrm{AL}$ & $\mathrm{CM}$ & NA & ML & $\mathrm{TH}$ \\
\hline 27 & $\mathrm{ML}$ & AL & $E$ & $\mathrm{ML}$ & MI & 73 & NA & $\mathrm{AL}$ & NA & $\mathrm{CL}$ & $\mathrm{TH}$ \\
\hline 28 & $\mathrm{ML}$ & $\mathrm{AL}$ & $\mathrm{CM}$ & $\mathrm{ML}$ & MI & 74 & $\mathrm{TH}$ & $\mathrm{AL}$ & NA & $\mathrm{CL}$ & $E$ \\
\hline 29 & PM & $\mathrm{NA}$ & $\mathrm{ML}$ & $\mathrm{ML}$ & $\mathrm{ML}$ & 75 & $\mathrm{TH}$ & $\mathrm{AL}$ & PM & ML & $\mathrm{ML}$ \\
\hline 30 & $\mathrm{CM}$ & $\mathrm{AL}$ & I & $\mathrm{ML}$ & $\mathrm{CL}$ & 76 & MI & $\mathrm{AL}$ & $C A$ & $\mathrm{CL}$ & $\mathrm{TH}$ \\
\hline 31 & $\mathrm{CM}$ & NA & $\mathrm{ML}$ & $\mathrm{ML}$ & $\mathrm{CL}$ & 77 & $\mathrm{AV}$ & $\mathrm{AL}$ & CA & $\mathrm{CL}$ & $\mathrm{TH}$ \\
\hline 32 & $\mathrm{Cl}$ & NA & PM & $\mathrm{ML}$ & $\mathrm{CL}$ & 78 & PM & $\mathrm{AL}$ & NA & TH & $\mathrm{TH}$ \\
\hline 33 & $\mathrm{AL}$ & $\mathrm{CA}$ & $\mathrm{CM}$ & $\mathrm{ML}$ & TH & 79 & $C A$ & NA & PM & $\mathrm{TH}$ & TO \\
\hline 34 & $\mathrm{AL}$ & $\mathrm{CA}$ & $\mathrm{AL}$ & $\mathrm{CL}$ & 1 & 80 & PM & $\mathrm{AL}$ & $\mathrm{CH}$ & $\mathrm{TH}$ & TO \\
\hline 35 & $E$ & NA & 1 & 1 & 1 & 81 & $C A$ & NA & $\mathrm{CM}$ & $\mathrm{CL}$ & TO \\
\hline 36 & $\mathrm{AL}$ & $\mathrm{CH}$ & $\mathrm{AL}$ & $\mathrm{CL}$ & $\mathrm{CL}$ & 82 & TO & NA & $\mathrm{CM}$ & $\mathrm{CL}$ & TO \\
\hline 37 & $E$ & $\mathrm{CH}$ & $\mathrm{AL}$ & $\mathrm{CL}$ & $\mathrm{CL}$ & 83 & PM & $\mathrm{AL}$ & PM & $\mathrm{ML}$ & TO \\
\hline 38 & $\mathrm{AL}$ & $\mathrm{CM}$ & $\mathrm{AL}$ & $\mathrm{CL}$ & $\mathrm{CL}$ & 84 & $C A$ & $\mathrm{CA}$ & $\mathrm{AL}$ & $\mathrm{CL}$ & TO \\
\hline 39 & NA & $\mathrm{AL}$ & $\mathrm{AL}$ & $\mathrm{CL}$ & $\mathrm{CL}$ & 85 & $\mathrm{CH}$ & $\mathrm{CA}$ & $\mathrm{AL}$ & $\mathrm{CL}$ & TO \\
\hline 40 & MI & $\mathrm{AL}$ & $\mathrm{AL}$ & $\mathrm{CL}$ & $\mathrm{CL}$ & 86 & $\mathrm{CM}$ & $C A$ & $\mathrm{AL}$ & $\mathrm{ML}$ & TO \\
\hline 41 & NA & $\mathrm{NA}$ & $\mathrm{AL}$ & $\mathrm{CL}$ & $\mathrm{ML}$ & 87 & $\mathrm{AL}$ & PM & NA & $\mathrm{CL}$ & TO \\
\hline 42 & NA & $\mathrm{AL}$ & $E$ & $\mathrm{CL}$ & $\mathrm{TH}$ & 88 & $\mathrm{AL}$ & $\mathrm{CM}$ & $\mathrm{AL}$ & $\mathrm{CL}$ & TO \\
\hline 43 & NA & $\mathrm{AL}$ & $\mathrm{AL}$ & TH & MI & 89 & $\mathrm{AL}$ & $\mathrm{TH}$ & $\mathrm{AL}$ & TH & TO \\
\hline 44 & $C A$ & $\mathrm{NA}$ & $\mathrm{AL}$ & $\mathrm{ML}$ & MI & 90 & $\mathrm{AL}$ & NA & $\mathrm{AL}$ & $\mathrm{TH}$ & TO \\
\hline 45 & $C A$ & PM & AL & $\mathrm{CL}$ & $E$ & & & & & & \\
\hline
\end{tabular}

Fuente: Elaboración Propia. 
Figura No 55: Distribución de Trabajo de Asentado de Ladrillo - Carta Balance ${ }^{\circ} 5$

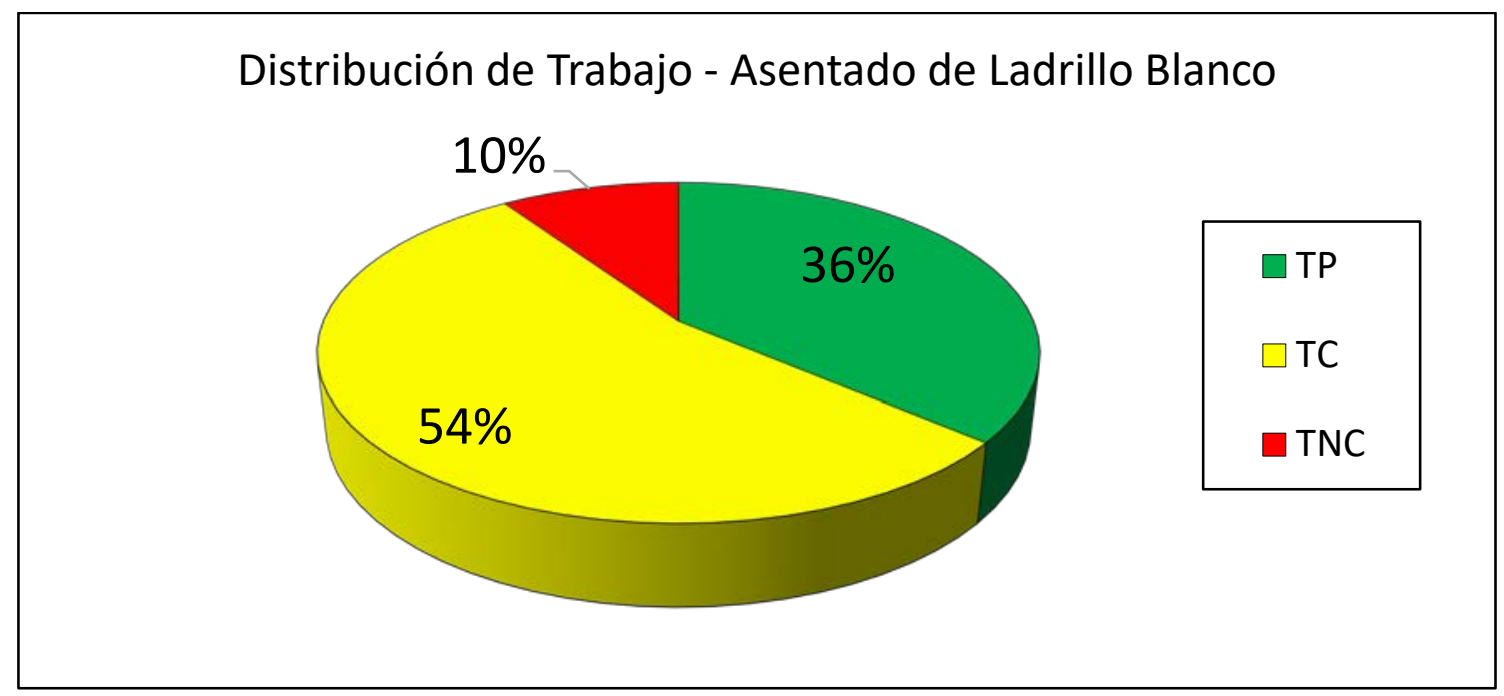

Fuente: Elaboración Propia.

Figura $N^{\circ}$ 56: Distribución de Trabajo Productivo - Carta Balance ${ }^{\circ} 5$

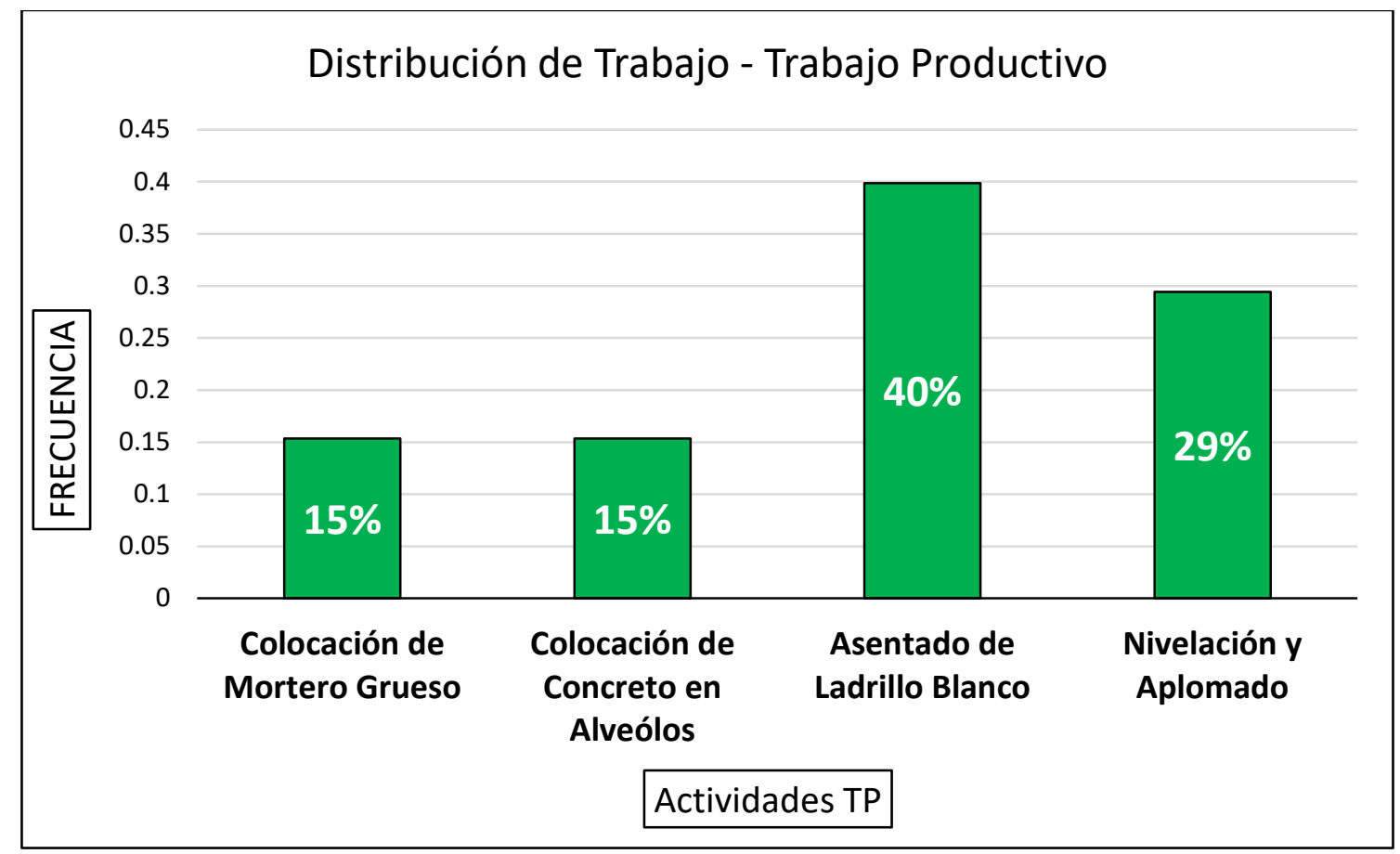

Fuente: Elaboración Propia. 
Figura $N^{\circ}$ 57: Distribución de Trabajo Contributorio - Carta Balance ${ }^{\circ} 5$

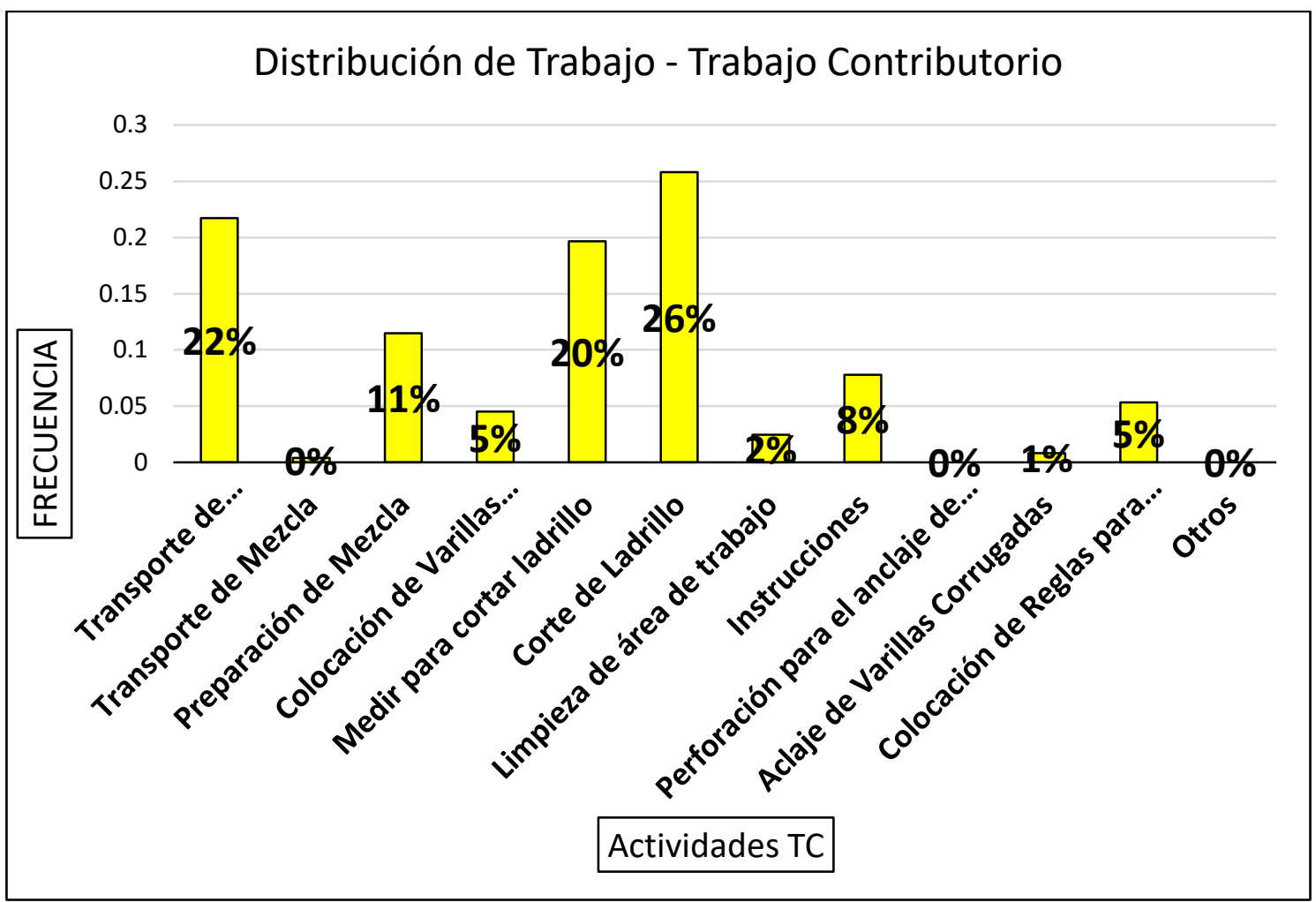

Fuente: Elaboración Propia.

Figura No 58: Distribución de Trabajo No Contributorio - Carta Balance ${ }^{\circ} 5$

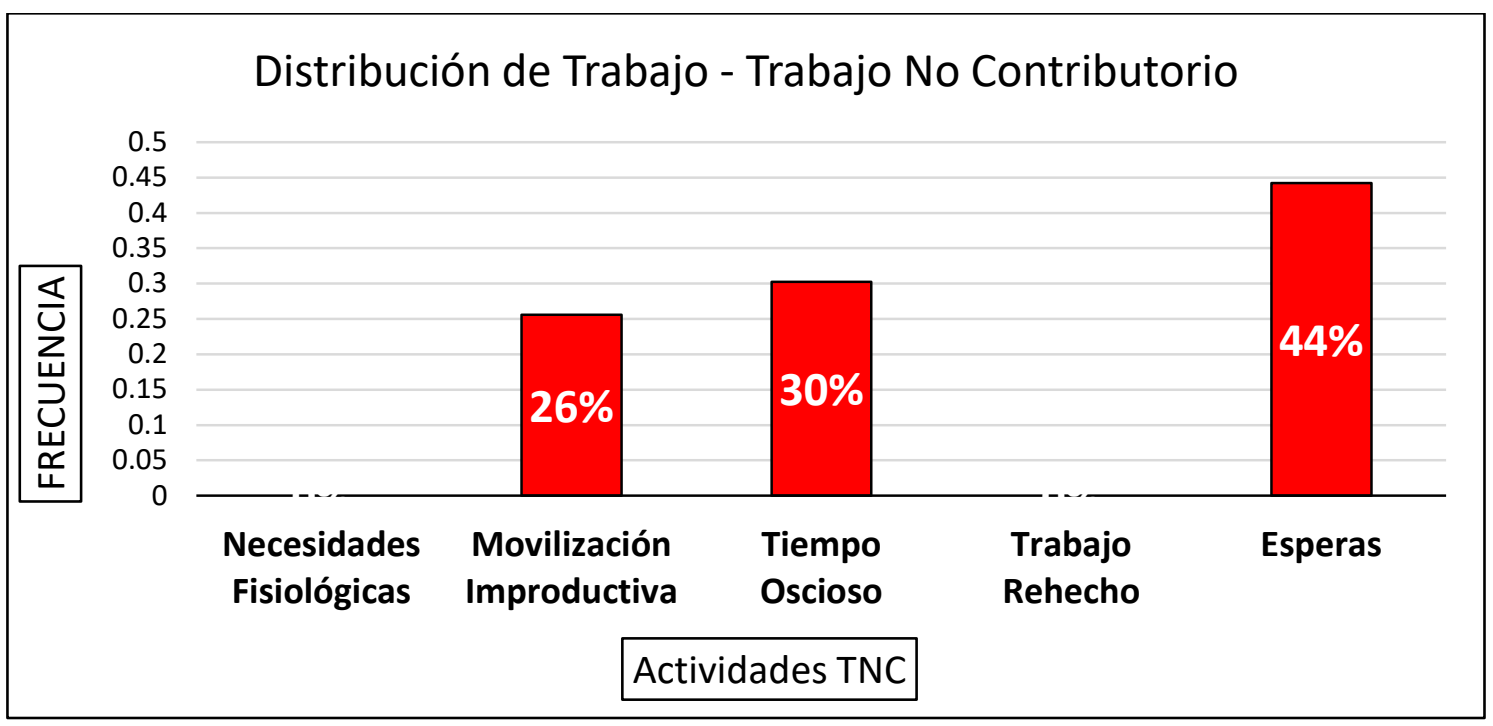

Fuente: Elaboración Propia. 
Tabla N ${ }^{\circ}$ 18: Carta Balance ${ }^{\circ} 6$ - Asentado de Ladrillo

\begin{tabular}{|c|c|c|c|c|c|c|c|c|c|c|c|}
\hline $\mathrm{N}^{0}$ & Obrero\#1 & Obrero \#2 & Obrero \#3 & Obrero \#4 & Obrero \#5 & 46 & $C R$ & $\mathrm{TH}$ & $\mathrm{CM}$ & $\mathrm{CL}$ & MI \\
\hline 1 & $C A$ & $\mathrm{AL}$ & $\mathrm{AL}$ & $\mathrm{ML}$ & $\mathrm{L}$ & 47 & $C R$ & $C R$ & $\mathrm{AL}$ & $\mathrm{ML}$ & $\mathrm{MI}$ \\
\hline 2 & MI & $\mathrm{NA}$ & $\mathrm{ML}$ & $\mathrm{CL}$ & $\mathrm{L}$ & 48 & $C R$ & $\mathrm{TH}$ & $\mathrm{AL}$ & $\mathrm{TH}$ & $\mathrm{MI}$ \\
\hline 3 & $\mathrm{MI}$ & NA & $\mathrm{ML}$ & $\mathrm{CL}$ & $\mathrm{L}$ & 49 & $C R$ & $\mathrm{TH}$ & NA & $\mathrm{ML}$ & $\mathrm{MI}$ \\
\hline 4 & MI & $\mathrm{CH}$ & $\mathrm{ML}$ & $\mathrm{CL}$ & $\mathrm{L}$ & 50 & TM & $\mathrm{L}$ & $\mathrm{AL}$ & $\mathrm{ML}$ & MI \\
\hline 5 & 1 & $\mathrm{CM}$ & 1 & $\mathrm{CL}$ & $L$ & 51 & $C R$ & $\mathrm{TH}$ & NA & $\mathrm{CL}$ & $\mathrm{TH}$ \\
\hline 6 & $\mathrm{CH}$ & NA & NA & $\mathrm{ML}$ & $L$ & 52 & $C R$ & $\mathrm{TH}$ & NA & $\mathrm{CL}$ & $\mathrm{CL}$ \\
\hline 7 & PM & $\mathrm{CM}$ & $\mathrm{AL}$ & $\mathrm{ML}$ & $\mathrm{CL}$ & 53 & $C R$ & $\mathrm{~L}$ & $\mathrm{AL}$ & $E$ & $\mathrm{CL}$ \\
\hline 8 & $\mathrm{CM}$ & $\mathrm{AL}$ & $\mathrm{AL}$ & $\mathrm{ML}$ & $\mathrm{TH}$ & 54 & $C R$ & $C R$ & $\mathrm{AL}$ & $\mathrm{CL}$ & $\mathrm{TH}$ \\
\hline 9 & $\mathrm{AL}$ & NA & $\mathrm{NA}$ & 1 & 1 & 55 & $\mathrm{NA}$ & $C R$ & NA & $\mathrm{CL}$ & $\mathrm{TH}$ \\
\hline 10 & NA & $\mathrm{NA}$ & NA & $\mathrm{TH}$ & $\mathrm{CL}$ & 56 & $\mathrm{NA}$ & $\mathrm{TH}$ & NA & $\mathrm{ML}$ & $\mathrm{TH}$ \\
\hline 11 & 1 & NA & NA & 1 & $\mathrm{TH}$ & 57 & $\mathrm{ML}$ & $C R$ & NA & $\mathrm{CL}$ & $\mathrm{TH}$ \\
\hline 12 & $\mathrm{AL}$ & $C A$ & NA & $\mathrm{ML}$ & $\mathrm{CA}$ & 58 & $\mathrm{ML}$ & $C R$ & PM & $\mathrm{CL}$ & $\mathrm{TH}$ \\
\hline 13 & $\mathrm{AL}$ & $C A$ & NA & $\mathrm{ML}$ & $C A$ & 59 & $\mathrm{ML}$ & $C R$ & $C A$ & $\mathrm{ML}$ & $\mathrm{TH}$ \\
\hline 14 & $\mathrm{CA}$ & $C A$ & PM & $\mathrm{ML}$ & $C A$ & 60 & $\mathrm{ML}$ & $C R$ & $C A$ & $\mathrm{ML}$ & $\mathrm{TH}$ \\
\hline 15 & $\mathrm{CA}$ & $L$ & $\mathrm{CA}$ & 1 & 1 & 61 & $\mathrm{ML}$ & $C R$ & $C A$ & $\mathrm{ML}$ & $\mathrm{L}$ \\
\hline 16 & $\mathrm{CA}$ & $\mathrm{CA}$ & $C A$ & $\mathrm{CL}$ & $\mathrm{CL}$ & 62 & $\mathrm{ML}$ & $C R$ & $C A$ & $\mathrm{CL}$ & $\mathrm{TH}$ \\
\hline 17 & $\mathrm{ML}$ & $\mathrm{CA}$ & PM & $\mathrm{TH}$ & $\mathrm{TH}$ & 63 & PM & $C R$ & $\mathrm{CM}$ & $\mathrm{CL}$ & $\mathrm{TH}$ \\
\hline 18 & $\mathrm{ML}$ & $\mathrm{CA}$ & $\mathrm{CM}$ & $\mathrm{CL}$ & $\mathrm{TH}$ & 64 & $\mathrm{CM}$ & $C R$ & $\mathrm{CM}$ & $\mathrm{CL}$ & $\mathrm{TH}$ \\
\hline 19 & $E$ & $L$ & $\mathrm{AL}$ & $\mathrm{CL}$ & $\mathrm{TH}$ & 65 & $\mathrm{CM}$ & PM & $\mathrm{AL}$ & $\mathrm{CL}$ & $\mathrm{TH}$ \\
\hline 20 & $\mathrm{ML}$ & $L$ & $\mathrm{AL}$ & $\mathrm{CL}$ & $\mathrm{TH}$ & 66 & $\mathrm{AL}$ & $\mathrm{ML}$ & $\mathrm{AL}$ & $\mathrm{ML}$ & $\mathrm{TH}$ \\
\hline 21 & $\mathrm{ML}$ & $L$ & $\mathrm{NA}$ & $\mathrm{ML}$ & $\mathrm{TH}$ & 67 & $\mathrm{AL}$ & 1 & NA & $E$ & $\mathrm{TH}$ \\
\hline 22 & $\mathrm{ML}$ & $L$ & 1 & 1 & $\mathrm{TH}$ & 68 & $\mathrm{AL}$ & $\mathrm{L}$ & $\mathrm{AL}$ & $\mathrm{CL}$ & $\mathrm{TH}$ \\
\hline 23 & $\mathrm{ML}$ & $L$ & 1 & $\mathrm{CL}$ & $\mathrm{TH}$ & 69 & $\mathrm{AL}$ & $\mathrm{L}$ & $\mathrm{AL}$ & $\mathrm{ML}$ & $\mathrm{TH}$ \\
\hline 24 & NA & $L$ & $\mathrm{AL}$ & $\mathrm{CL}$ & $\mathrm{TH}$ & 70 & 1 & $\mathrm{~L}$ & $\mathrm{AL}$ & $\mathrm{ML}$ & $\mathrm{TH}$ \\
\hline 25 & $E$ & $L$ & $\mathrm{AL}$ & $\mathrm{CL}$ & $\mathrm{TH}$ & 71 & $\mathrm{NA}$ & $\mathrm{ML}$ & NA & $E$ & $\mathrm{TH}$ \\
\hline 26 & PM & NA & $\mathrm{AL}$ & $E$ & TH & 72 & $\mathrm{AL}$ & NA & $\mathrm{AL}$ & 1 & $\mathrm{TH}$ \\
\hline 27 & $\mathrm{CM}$ & NA & $E$ & $\mathrm{CL}$ & $\mathrm{TH}$ & 73 & $\mathrm{NA}$ & PM & $\mathrm{AL}$ & $\mathrm{CL}$ & $\mathrm{TH}$ \\
\hline 28 & NA & NA & $\mathrm{AL}$ & $\mathrm{CL}$ & $\mathrm{TH}$ & 74 & $\mathrm{NA}$ & $\mathrm{CM}$ & $\mathrm{AL}$ & $\mathrm{CL}$ & $\mathrm{TH}$ \\
\hline 29 & $\mathrm{AL}$ & NA & $\mathrm{AL}$ & TH & $\mathrm{TH}$ & 75 & $\mathrm{PM}$ & $\mathrm{CM}$ & NA & $\mathrm{TH}$ & $\mathrm{L}$ \\
\hline 30 & NA & $L$ & $\mathrm{AL}$ & $\mathrm{CL}$ & TH & 76 & $\mathrm{CA}$ & $\mathrm{AL}$ & NA & $\mathrm{TH}$ & $\mathrm{L}$ \\
\hline 31 & $\mathrm{AL}$ & $L$ & $\mathrm{AL}$ & $\mathrm{TH}$ & $\mathrm{TH}$ & 77 & $\mathrm{ML}$ & $\mathrm{AL}$ & $\mathrm{TM}$ & $E$ & $\mathrm{~L}$ \\
\hline 32 & $\mathrm{AL}$ & $L$ & 1 & $\mathrm{CL}$ & $\mathrm{TH}$ & 78 & $\mathrm{ML}$ & $\mathrm{AL}$ & $\mathrm{PM}$ & $\mathrm{ML}$ & $\mathrm{L}$ \\
\hline 33 & NA & $L$ & $\mathrm{NA}$ & $\mathrm{CL}$ & $\mathrm{TH}$ & 79 & $\mathrm{ML}$ & $\mathrm{AL}$ & $C A$ & $\mathrm{CL}$ & $\mathrm{L}$ \\
\hline 34 & $\mathrm{PM}$ & $\mathrm{L}$ & AV & $\mathrm{Cl}$ & $\mathrm{L}$ & 80 & $\mathrm{ML}$ & $\mathrm{AL}$ & $C A$ & $\mathrm{CL}$ & $\mathrm{L}$ \\
\hline 35 & $C A$ & $L$ & $\mathrm{AV}$ & $\mathrm{CL}$ & $L$ & 81 & PM & $\mathrm{ML}$ & NA & $\mathrm{CL}$ & $\mathrm{L}$ \\
\hline 36 & $L$ & $L$ & $\mathrm{NA}$ & $\mathrm{CL}$ & $L$ & 82 & $\mathrm{CM}$ & $\mathrm{CL}$ & $\mathrm{CH}$ & $\mathrm{CL}$ & $\mathrm{L}$ \\
\hline 37 & $L$ & $L$ & NA & $\mathrm{ML}$ & $L$ & 83 & $\mathrm{AL}$ & $\mathrm{TH}$ & $\mathrm{CM}$ & $\mathrm{ML}$ & $\mathrm{L}$ \\
\hline 38 & $L$ & $L$ & NA & $\mathrm{CL}$ & $\mathrm{L}$ & 84 & $\mathrm{AL}$ & $\mathrm{AL}$ & $\mathrm{CM}$ & $\mathrm{ML}$ & $\mathrm{L}$ \\
\hline 39 & TH & $L$ & TH & $\mathrm{ML}$ & TH & 85 & $\mathrm{AL}$ & $\mathrm{AL}$ & $\mathrm{AL}$ & $\mathrm{CL}$ & $\mathrm{L}$ \\
\hline 40 & TH & $L$ & $\mathrm{TH}$ & $\mathrm{ML}$ & $\mathrm{TH}$ & 86 & NA & NA & $\mathrm{AL}$ & $\mathrm{ML}$ & $\mathrm{L}$ \\
\hline 41 & $L$ & $L$ & $\mathrm{CA}$ & $\mathrm{ML}$ & $\mathrm{TH}$ & 87 & $\mathrm{AL}$ & NA & $\mathrm{AL}$ & $\mathrm{ML}$ & $\mathrm{L}$ \\
\hline 42 & $C R$ & $\mathrm{~L}$ & $C A$ & $\mathrm{CL}$ & MI & 88 & $\mathrm{AL}$ & NA & $\mathrm{ML}$ & $\mathrm{ML}$ & $\mathrm{L}$ \\
\hline 43 & $\mathrm{CR}$ & $\mathrm{L}$ & $\mathrm{CH}$ & TH & $\mathrm{MI}$ & 89 & $\mathrm{NA}$ & $\mathrm{TH}$ & $\mathrm{ML}$ & $\mathrm{CL}$ & $\mathrm{L}$ \\
\hline 44 & $C R$ & $\mathrm{~L}$ & $\mathrm{CH}$ & $E$ & $\mathrm{MI}$ & 90 & $\mathrm{NA}$ & $\mathrm{TM}$ & $\mathrm{AL}$ & $\mathrm{CL}$ & $\mathrm{L}$ \\
\hline 45 & $C R$ & TH & $\mathrm{CM}$ & TH & $\mathrm{MI}$ & & & & & & \\
\hline
\end{tabular}

Fuente: Elaboración Propia. 
Figura No 59: Distribución de Trabajo de Asentado de Ladrillo - Carta Balance ${ }^{\circ} 6$

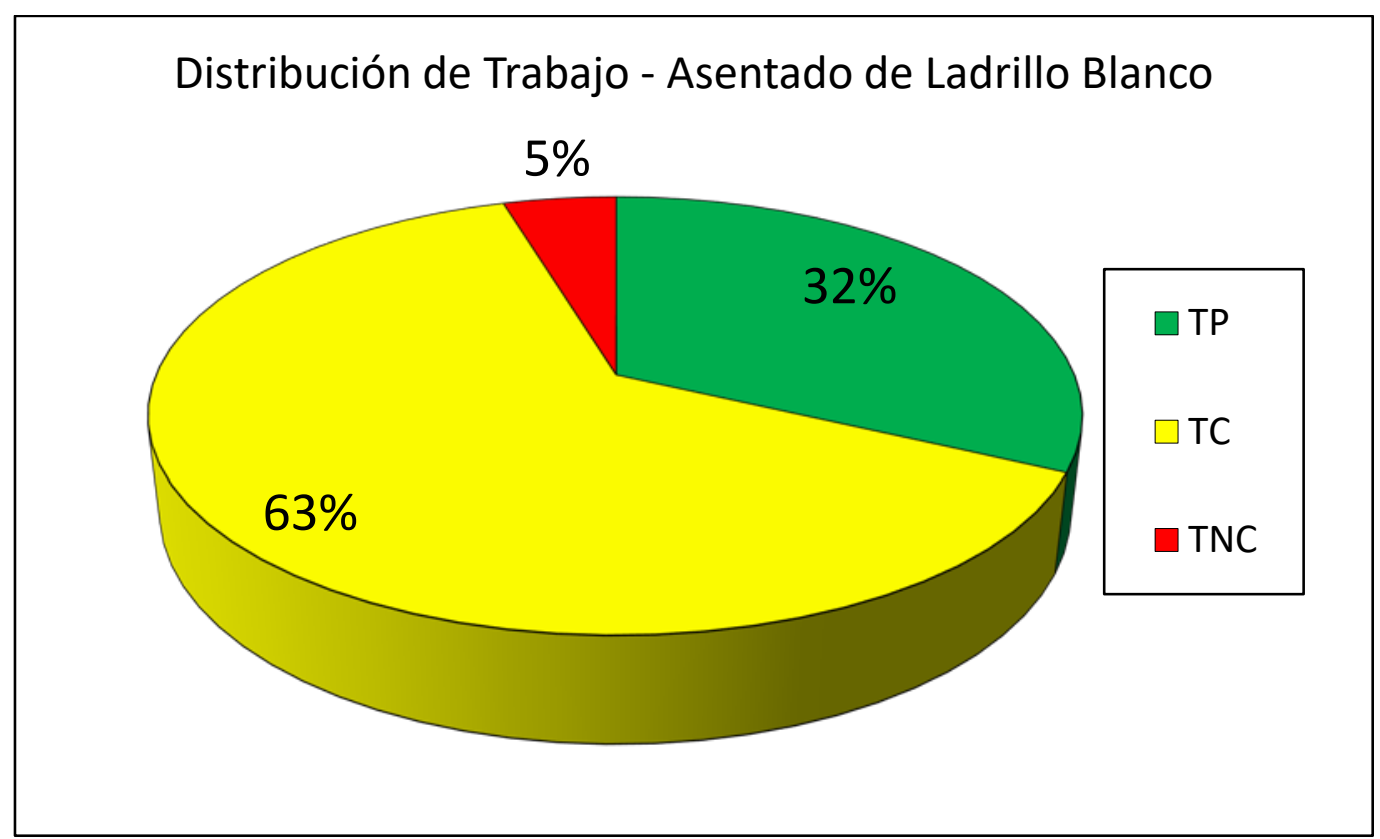

Fuente: Elaboración Propia.

Figura $N^{\circ}$ 60: Distribución de Trabajo Productivo - Carta Balance ${ }^{\circ} 6$

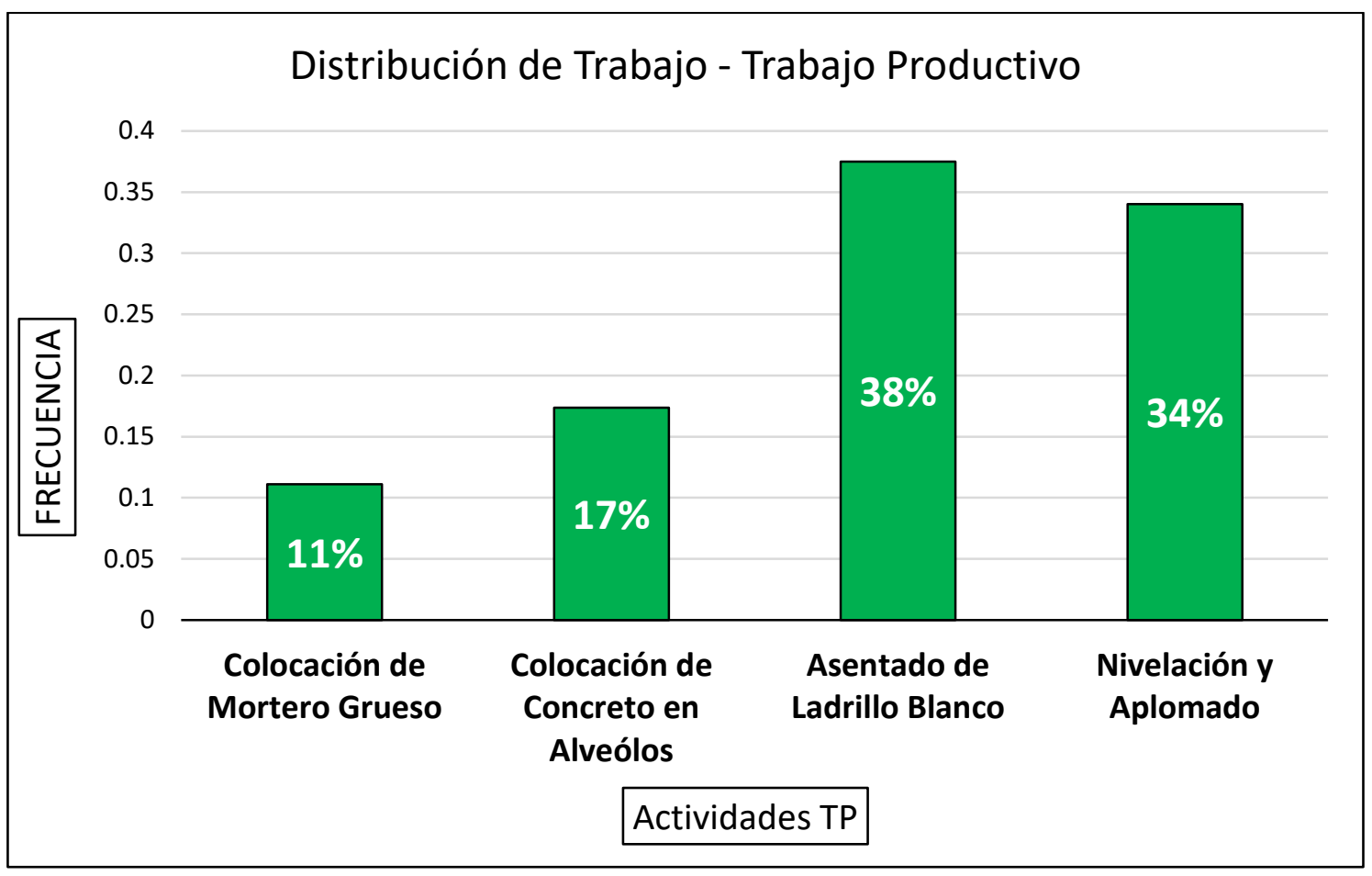

Fuente: Elaboración Propia. 
Figura $N^{\circ}$ 61: Distribución de Trabajo Contributorio - Carta Balance ${ }^{\circ} 6$

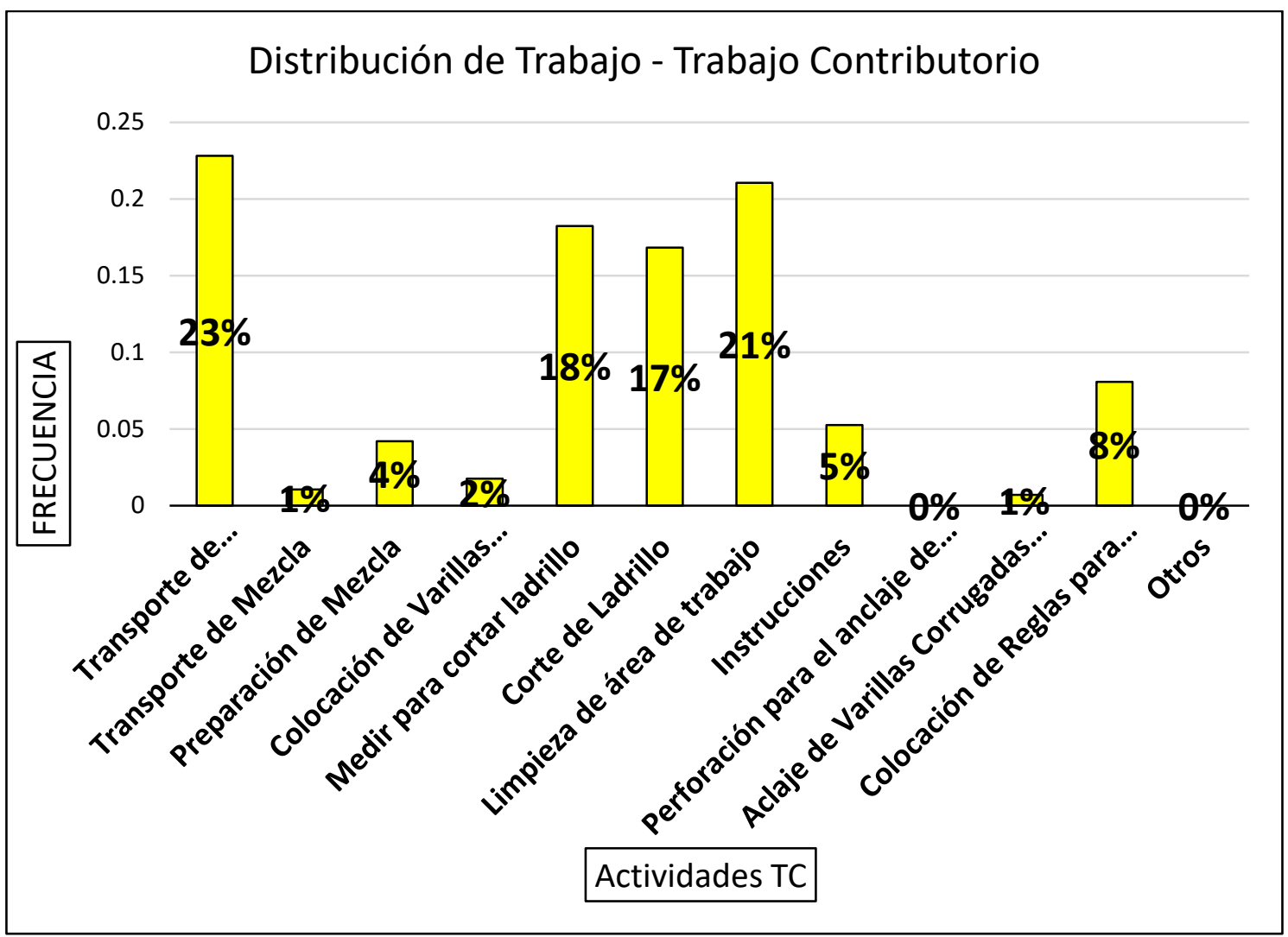

Fuente: Elaboración Propia.

Figura $N^{\circ}$ 62: Distribución de Trabajo No Contributorio - Carta Balance ${ }^{\circ} 6$

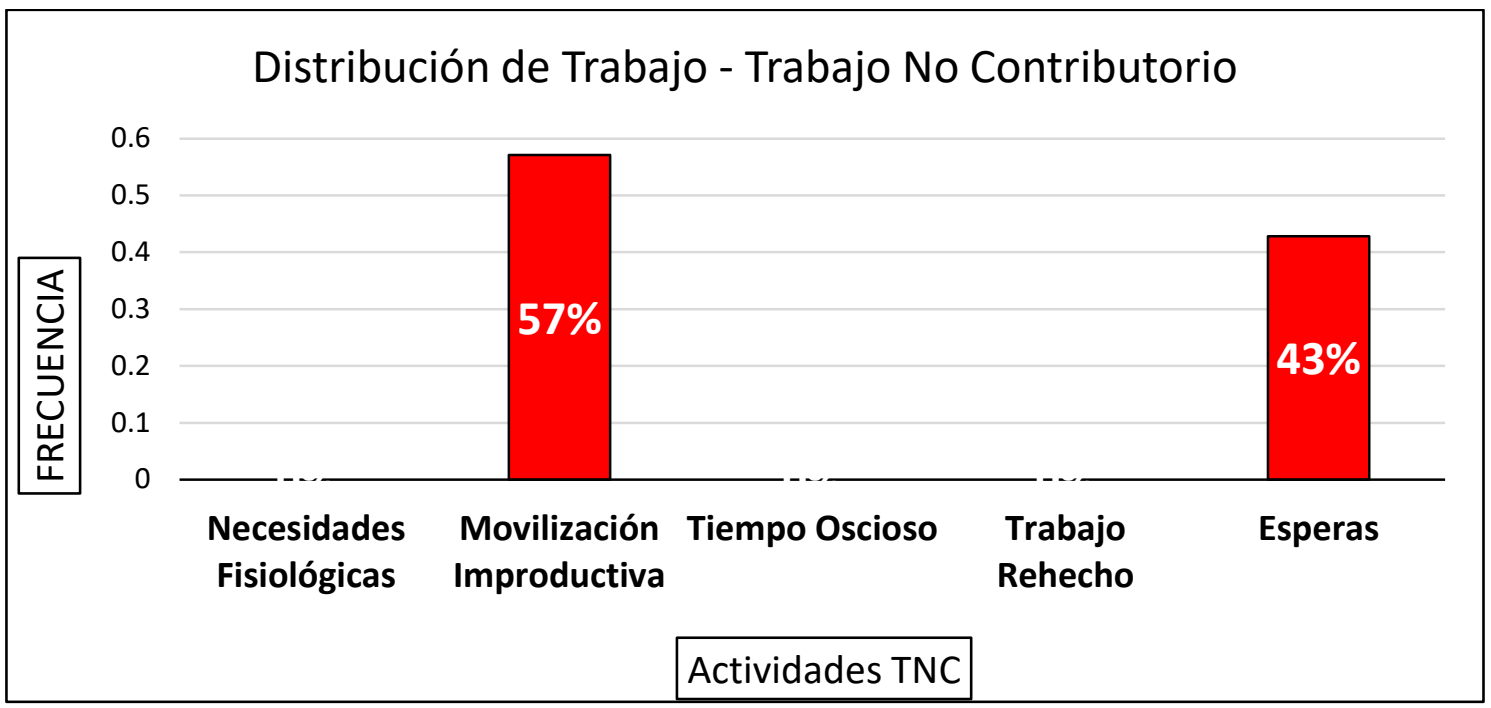

Fuente: Elaboración Propia. 
Tabla N 19: Carta Balance ${ }^{\circ} 7$ - Asentado de Ladrillo

\begin{tabular}{|c|c|c|c|c|c|c|c|c|c|c|c|}
\hline $\mathrm{N}^{0}$ & Obrero\#1 & Obrero\#2 & Obrero\#3 & Obrero\#4 & Obrero \#5 & 46 & $\mathrm{CM}$ & $\mathrm{AL}$ & NA & $\mathrm{L}$ & $\mathrm{TM}$ \\
\hline 1 & NA & $C A$ & NA & $\mathrm{CL}$ & L & 47 & $\mathrm{AL}$ & $\mathrm{TM}$ & $\mathrm{CA}$ & $\mathrm{L}$ & L \\
\hline 2 & $\mathrm{CM}$ & $C A$ & PM & $\mathrm{ML}$ & L & 48 & NA & PM & PM & $\mathrm{L}$ & $\mathrm{TH}$ \\
\hline 3 & $\mathrm{AL}$ & $C A$ & $\mathrm{CM}$ & $\mathrm{ML}$ & $L$ & 49 & NA & $\mathrm{CA}$ & $\mathrm{CA}$ & $\mathrm{L}$ & $\mathrm{TH}$ \\
\hline 4 & $\mathrm{AL}$ & PM & $\mathrm{AL}$ & $\mathrm{TH}$ & $L$ & 50 & $\mathrm{AL}$ & $\mathrm{CA}$ & $\mathrm{CA}$ & $L$ & $\mathrm{TH}$ \\
\hline 5 & $\mathrm{AL}$ & $\mathrm{TH}$ & $\mathrm{AL}$ & $\mathrm{TH}$ & $L$ & 51 & $\mathrm{AL}$ & $\mathrm{CA}$ & $\mathrm{CA}$ & $\mathrm{L}$ & $\mathrm{TH}$ \\
\hline 6 & $\mathrm{AL}$ & $\mathrm{CH}$ & $\mathrm{ML}$ & $\mathrm{ML}$ & $L$ & 52 & $\mathrm{AL}$ & PM & $\mathrm{CA}$ & $\mathrm{L}$ & $\mathrm{TH}$ \\
\hline 7 & $\mathrm{AL}$ & $\mathrm{NA}$ & $\mathrm{ML}$ & $\mathrm{CL}$ & L & 53 & $\mathrm{AL}$ & $\mathrm{CA}$ & $L$ & $\mathrm{CL}$ & $\mathrm{TH}$ \\
\hline 8 & NA & $C A$ & $E$ & $\mathrm{CL}$ & $\mathrm{TH}$ & 54 & NA & NA & $\mathrm{CA}$ & $\mathrm{CL}$ & $\mathrm{TH}$ \\
\hline 9 & PM & $\mathrm{AL}$ & $\mathrm{AL}$ & $\mathrm{CL}$ & $\mathrm{TH}$ & 55 & PM & $\mathrm{CA}$ & $\mathrm{CA}$ & $\mathrm{CL}$ & $\mathrm{TH}$ \\
\hline 10 & $C A$ & $\mathrm{CM}$ & $\mathrm{ML}$ & $\mathrm{CL}$ & $\mathrm{TH}$ & 56 & $\mathrm{CM}$ & $\mathrm{CA}$ & $L$ & $\mathrm{CL}$ & $\mathrm{TH}$ \\
\hline 11 & $C A$ & $\mathrm{CM}$ & $\mathrm{CM}$ & $\mathrm{CL}$ & $\mathrm{TH}$ & 57 & $\mathrm{CM}$ & L & $\mathrm{L}$ & $\mathrm{ML}$ & $\mathrm{TH}$ \\
\hline 12 & $C A$ & $\mathrm{CM}$ & $\mathrm{AL}$ & $\mathrm{TH}$ & $\mathrm{TH}$ & 58 & $\mathrm{AL}$ & $\mathrm{L}$ & $L$ & $\mathrm{CL}$ & $\mathrm{TH}$ \\
\hline 13 & PM & $\mathrm{AL}$ & NA & $\mathrm{CL}$ & $\mathrm{TH}$ & 59 & $\mathrm{AL}$ & 1 & $L$ & $\mathrm{TH}$ & $\mathrm{TH}$ \\
\hline 14 & $\mathrm{CA}$ & $\mathrm{AL}$ & PM & $\mathrm{CL}$ & $\mathrm{TH}$ & 60 & $\mathrm{AL}$ & $\mathrm{L}$ & $\mathrm{L}$ & $\mathrm{CL}$ & $\mathrm{TH}$ \\
\hline 15 & PM & $\mathrm{AL}$ & $\mathrm{CA}$ & $\mathrm{ML}$ & $\mathrm{TH}$ & 61 & $\mathrm{AL}$ & $\mathrm{L}$ & $L$ & $\mathrm{TH}$ & $\mathrm{TH}$ \\
\hline 16 & NA & $\mathrm{NA}$ & $C A$ & $\mathrm{CL}$ & $\mathrm{TH}$ & 62 & NA & $L$ & $\mathrm{TH}$ & $\mathrm{CL}$ & $\mathrm{TH}$ \\
\hline 17 & $\mathrm{TH}$ & $\mathrm{AL}$ & $\mathrm{ML}$ & $\mathrm{TH}$ & $\mathrm{TH}$ & 63 & NA & 1 & 1 & $\mathrm{CL}$ & $\mathrm{TH}$ \\
\hline 18 & $\mathrm{CM}$ & $\mathrm{AL}$ & $\mathrm{NA}$ & $\mathrm{TH}$ & $\mathrm{TH}$ & 64 & $\mathrm{PM}$ & $E$ & $L$ & $\mathrm{CL}$ & $\mathrm{TH}$ \\
\hline 19 & $\mathrm{CM}$ & $\mathrm{AL}$ & $\mathrm{ML}$ & $\mathrm{TH}$ & $\mathrm{TH}$ & 65 & $C A$ & NA & $L$ & $\mathrm{CL}$ & $\mathrm{TH}$ \\
\hline 20 & $\mathrm{AL}$ & $\mathrm{AL}$ & $\mathrm{CH}$ & $\mathrm{ML}$ & $\mathrm{TH}$ & 66 & $C A$ & NA & $\mathrm{TH}$ & $\mathrm{CL}$ & $\mathrm{TH}$ \\
\hline 21 & $\mathrm{AL}$ & $\mathrm{AL}$ & $\mathrm{MI}$ & $\mathrm{CL}$ & $\mathrm{TH}$ & 67 & $C A$ & $L$ & $\mathrm{TH}$ & $\mathrm{CL}$ & $\mathrm{TH}$ \\
\hline 22 & 1 & $\mathrm{ML}$ & MI & $\mathrm{CL}$ & TH & 68 & $C A$ & L & $\mathrm{TH}$ & $\mathrm{CL}$ & $\mathrm{TH}$ \\
\hline 23 & 1 & $\mathrm{AL}$ & $\mathrm{CM}$ & $\mathrm{CL}$ & TH & 69 & $E$ & $\mathrm{TH}$ & $\mathrm{TH}$ & $\mathrm{TH}$ & $\mathrm{TH}$ \\
\hline 24 & $\mathrm{AL}$ & $\mathrm{AL}$ & PM & $\mathrm{TH}$ & $\mathrm{TH}$ & 70 & $\mathrm{AV}$ & $\mathrm{TH}$ & $\mathrm{TH}$ & I & $\mathrm{TH}$ \\
\hline 25 & $\mathrm{NA}$ & $\mathrm{NA}$ & $\mathrm{AL}$ & $\mathrm{TH}$ & $\mathrm{TH}$ & 71 & $\mathrm{MI}$ & $\mathrm{TH}$ & $\mathrm{TH}$ & $E$ & $\mathrm{TH}$ \\
\hline 26 & $\mathrm{NA}$ & $\mathrm{NA}$ & $\mathrm{ML}$ & $\mathrm{ML}$ & $\mathrm{TH}$ & 72 & $\mathrm{Ml}$ & $C R$ & $L$ & $E$ & $\mathrm{TH}$ \\
\hline 27 & PM & $\mathrm{NA}$ & $\mathrm{CM}$ & $\mathrm{CL}$ & $\mathrm{TH}$ & 73 & $\mathrm{MI}$ & $C R$ & L & $E$ & $\mathrm{TH}$ \\
\hline 28 & $\mathrm{CM}$ & $\mathrm{NA}$ & $\mathrm{CM}$ & TH & TH & 74 & $\mathrm{AV}$ & $C R$ & $\mathrm{~L}$ & $E$ & $\mathrm{TH}$ \\
\hline 29 & $\mathrm{AL}$ & $C A$ & $\mathrm{AL}$ & $\mathrm{ML}$ & $\mathrm{TH}$ & 75 & $\mathrm{AV}$ & $C R$ & $\mathrm{TH}$ & $E$ & $\mathrm{TH}$ \\
\hline 30 & $\mathrm{AL}$ & $C A$ & $\mathrm{NA}$ & $\mathrm{CL}$ & $\mathrm{TH}$ & 76 & $\mathrm{AV}$ & $C R$ & $C R$ & $E$ & $\mathrm{TH}$ \\
\hline 31 & $\mathrm{AL}$ & $C A$ & $\mathrm{AL}$ & $\mathrm{TH}$ & $\mathrm{TH}$ & 77 & $\mathrm{PM}$ & $C R$ & $C R$ & $E$ & $\mathrm{TH}$ \\
\hline 32 & $\mathrm{AL}$ & PM & $\mathrm{AL}$ & $\mathrm{ML}$ & $\mathrm{TH}$ & 78 & $\mathrm{PM}$ & $C R$ & $C R$ & $E$ & $\mathrm{TH}$ \\
\hline 33 & NA & $C A$ & $\mathrm{PM}$ & $\mathrm{CL}$ & $\mathrm{TH}$ & 79 & $C A$ & $C R$ & $C R$ & $\mathrm{CL}$ & $\mathrm{TH}$ \\
\hline 34 & PM & $\mathrm{CM}$ & $\mathrm{CA}$ & TH & TH & 80 & CA & $\mathrm{L}$ & $C R$ & $\mathrm{CL}$ & $\mathrm{L}$ \\
\hline 35 & $\mathrm{CA}$ & $E$ & $C A$ & $\mathrm{CL}$ & $\mathrm{TH}$ & 81 & $C R$ & $E$ & $C R$ & $\mathrm{CL}$ & $L$ \\
\hline 36 & $C A$ & $\mathrm{AL}$ & $C A$ & $\mathrm{ML}$ & $\mathrm{TH}$ & 82 & $C R$ & $L$ & $C R$ & $\mathrm{CL}$ & $L$ \\
\hline 37 & $C A$ & $\mathrm{AL}$ & $\mathrm{ML}$ & $\mathrm{ML}$ & $\mathrm{TH}$ & 83 & $C R$ & $L$ & $C R$ & $\mathrm{CL}$ & $L$ \\
\hline 38 & $\mathrm{CA}$ & $\mathrm{AL}$ & NA & $\mathrm{CL}$ & $\mathrm{TH}$ & 84 & $\mathrm{TH}$ & $\mathrm{TH}$ & NA & $\mathrm{ML}$ & $L$ \\
\hline 39 & $C A$ & $C A$ & $L$ & $\mathrm{CL}$ & TH & 85 & NA & $E$ & $E$ & $\mathrm{CL}$ & $L$ \\
\hline 40 & L & $C A$ & $E$ & 1 & 1 & 86 & $\mathrm{PM}$ & $E$ & $\mathrm{TH}$ & $\mathrm{CL}$ & L \\
\hline 41 & $\mathrm{CH}$ & $\mathrm{CM}$ & $\mathrm{TH}$ & $L$ & $L$ & 87 & $\mathrm{CM}$ & $\mathrm{TH}$ & $\mathrm{CM}$ & $E$ & $L$ \\
\hline 42 & $\mathrm{CH}$ & $\mathrm{CM}$ & $\mathrm{CM}$ & $\mathrm{TH}$ & $L$ & 88 & $\mathrm{CM}$ & $\mathrm{ML}$ & $\mathrm{CM}$ & $\mathrm{CL}$ & $L$ \\
\hline 43 & NA & $\mathrm{CM}$ & $\mathrm{CM}$ & $\mathrm{TH}$ & $L$ & 89 & $\mathrm{AL}$ & $L$ & $\mathrm{CM}$ & $\mathrm{CL}$ & $L$ \\
\hline 44 & MI & $\mathrm{NA}$ & $\mathrm{AL}$ & $\mathrm{TH}$ & $L$ & 90 & $\mathrm{AL}$ & $\mathrm{L}$ & $\mathrm{AL}$ & $\mathrm{CL}$ & $L$ \\
\hline 45 & PM & $\mathrm{AL}$ & NA & L & TM & & & & & & \\
\hline
\end{tabular}

Fuente: Elaboración Propia. 
Figura $N^{\circ}$ 63: Distribución de Trabajo de Asentado de Ladrillo - Carta Balance ${ }^{\circ} 7$

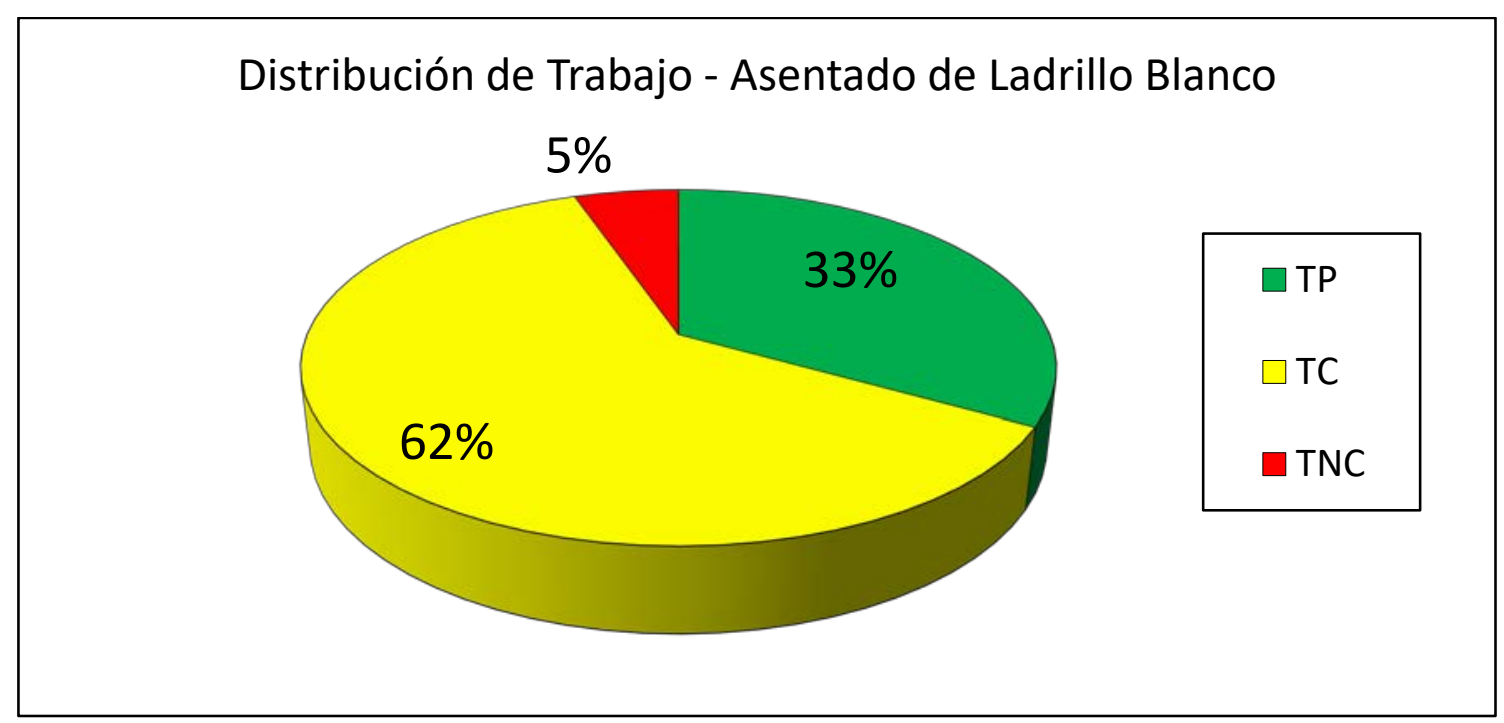

Fuente: Elaboración Propia.

Figura $N^{\circ}$ 64: Distribución de Trabajo Productivo - Carta Balance ${ }^{\circ} 7$

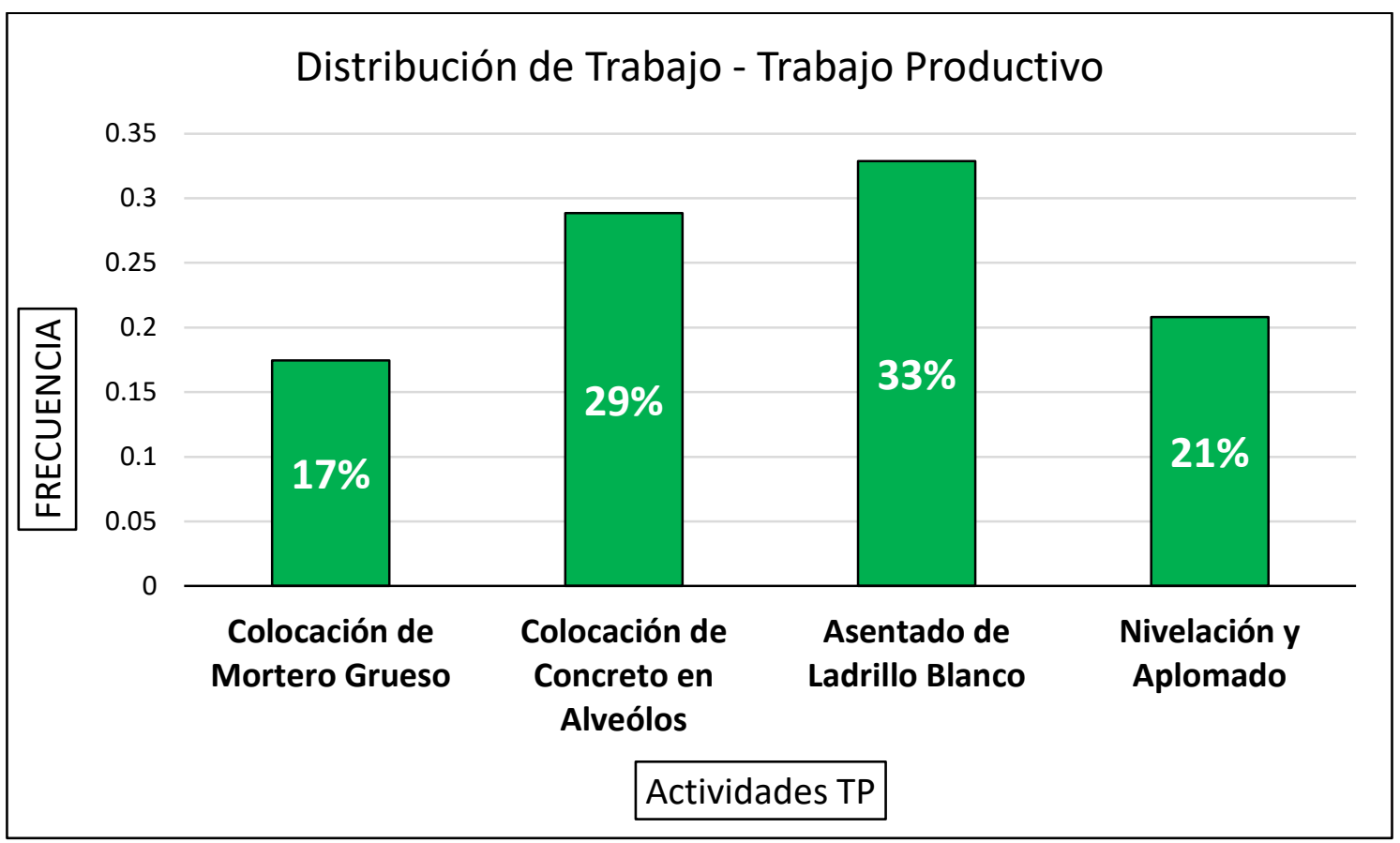

Fuente: Elaboración Propia. 
Figura $N^{\circ}$ 65: Distribución de Trabajo Contributorio - Carta Balance ${ }^{\circ} 7$

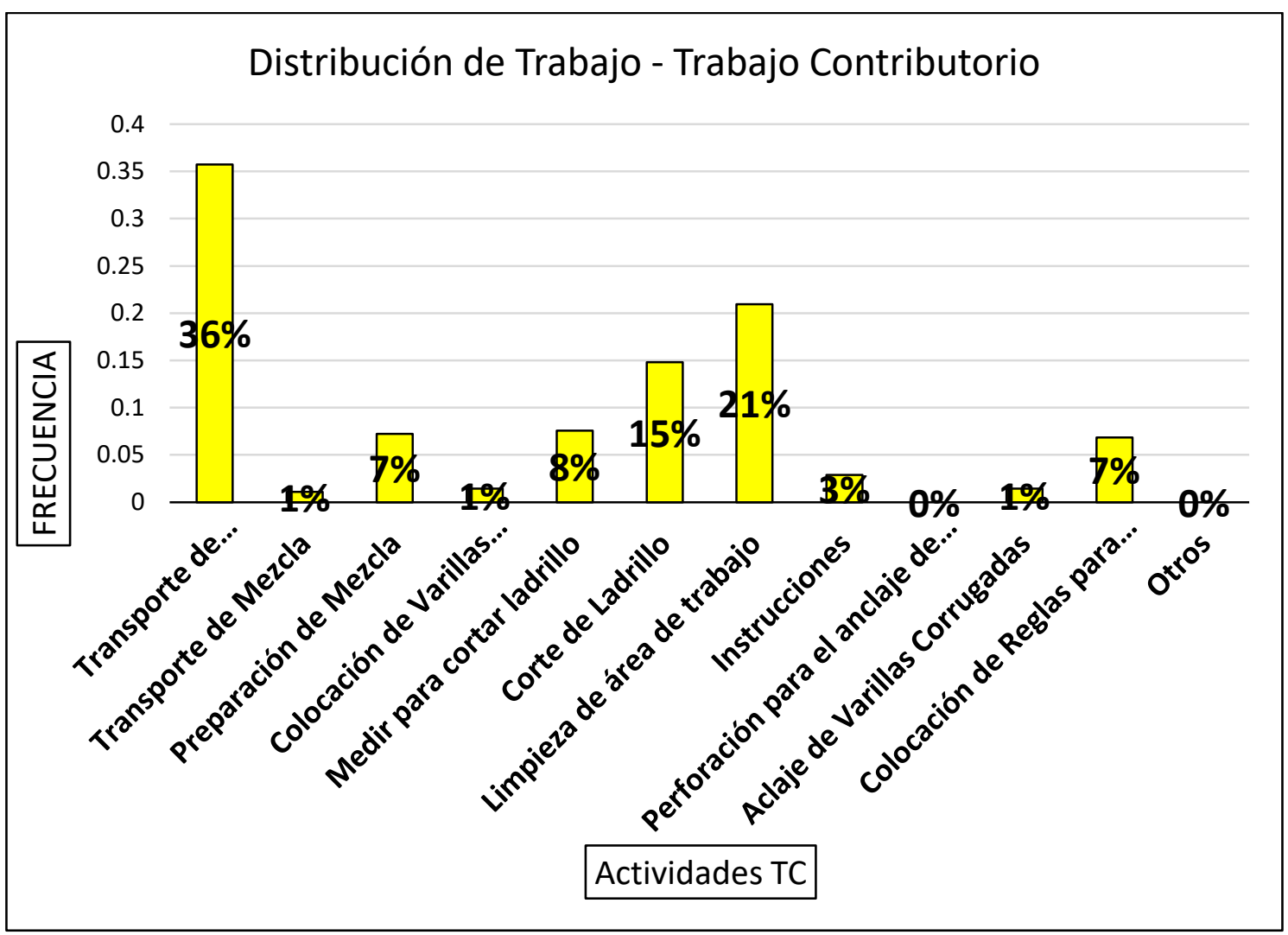

Fuente: Elaboración Propia.

Figura $N^{\circ}$ 66: Distribución de Trabajo No Contributorio - Carta Balance ${ }^{\circ} 7$

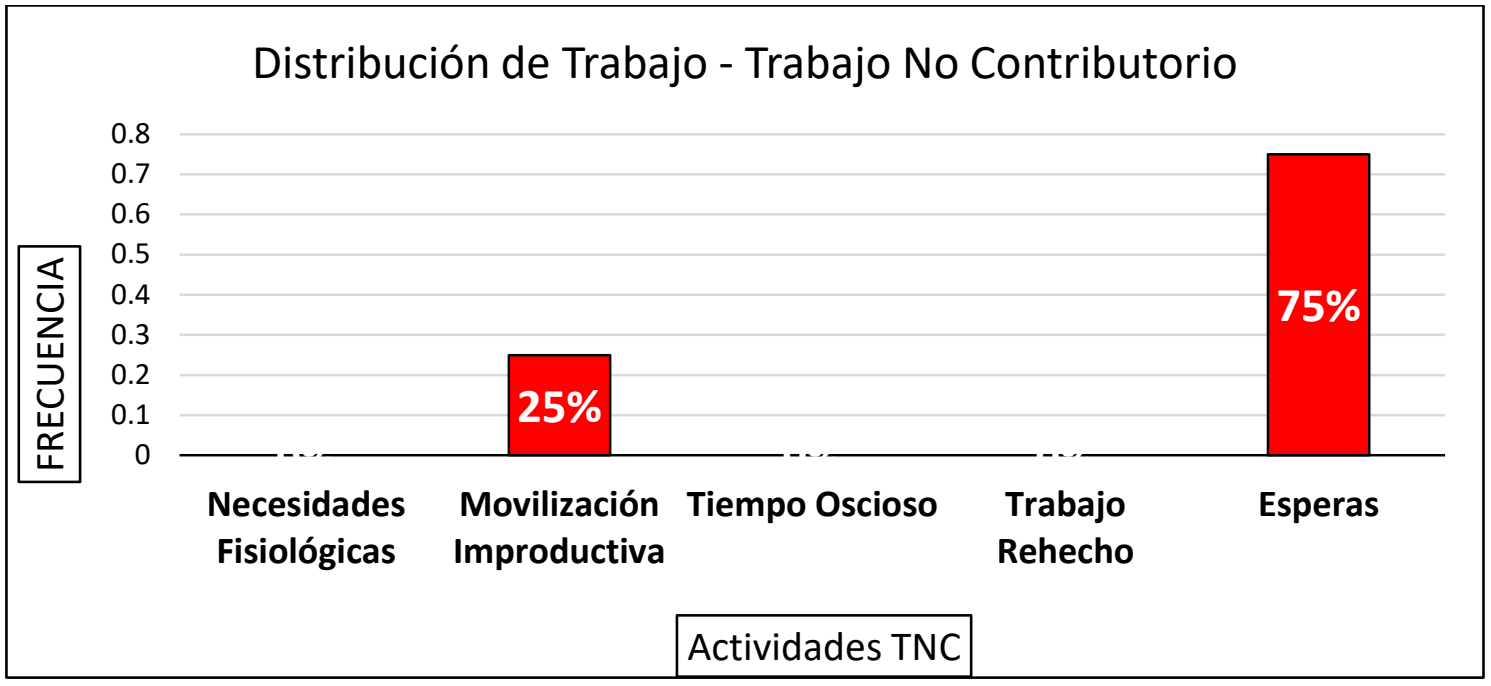

Fuente: Elaboración Propia. 
Tabla N 20: Carta Balance ${ }^{\circ} 8$ - Asentado de Ladrillo

\begin{tabular}{|c|c|c|c|c|c|c|c|c|c|c|c|}
\hline $\mathrm{N}^{0}$ & Obrero\#1 & Obrero\#2 & Obrero\#3 & Obrero\#4 & Obrero \#5 & 46 & NA & $\mathrm{AL}$ & MI & ML & $\mathrm{L}$ \\
\hline 1 & $\mathrm{AL}$ & $C R$ & $\mathrm{AL}$ & $\mathrm{CL}$ & L & 47 & PM & $E$ & NA & $\mathrm{CL}$ & $\mathrm{TH}$ \\
\hline 2 & NA & $C R$ & PM & $\mathrm{CL}$ & $\mathrm{L}$ & 48 & $\mathrm{CM}$ & $E$ & NA & $\mathrm{CL}$ & $\mathrm{TH}$ \\
\hline 3 & $\mathrm{NA}$ & $C R$ & $\mathrm{AL}$ & $\mathrm{TH}$ & $L$ & 49 & $\mathrm{CM}$ & $E$ & $C R$ & $\mathrm{CL}$ & $\mathrm{CL}$ \\
\hline 4 & PM & $C R$ & $\mathrm{AL}$ & $\mathrm{CL}$ & L & 50 & $\mathrm{AL}$ & $\mathrm{AL}$ & $C R$ & TH & $\mathrm{TH}$ \\
\hline 5 & $\mathrm{CM}$ & I & $\mathrm{AL}$ & $\mathrm{CL}$ & L & 51 & $\mathrm{AL}$ & $E$ & $C R$ & $\mathrm{CL}$ & $\mathrm{TH}$ \\
\hline 6 & $\mathrm{CL}$ & 1 & NA & $E$ & $L$ & 52 & $\mathrm{AL}$ & $E$ & $C R$ & $\mathrm{CL}$ & $\mathrm{CL}$ \\
\hline 7 & NA & 1 & NA & $\mathrm{CL}$ & $L$ & 53 & $\mathrm{~L}$ & $\mathrm{AL}$ & $C R$ & $E$ & $E$ \\
\hline 8 & $\mathrm{AL}$ & $C R$ & NA & $\mathrm{CL}$ & $L$ & 54 & $\mathrm{~L}$ & $E$ & $C R$ & ML & $\mathrm{TH}$ \\
\hline 9 & NA & 1 & $E$ & $\mathrm{ML}$ & $\mathrm{L}$ & 55 & $\mathrm{AL}$ & $E$ & $C R$ & TH & L \\
\hline 10 & $\mathrm{AL}$ & 1 & $C A$ & $\mathrm{CL}$ & L & 56 & $\mathrm{AL}$ & $\mathrm{CM}$ & $\mathrm{TH}$ & $\mathrm{TH}$ & L \\
\hline 11 & NA & I & PM & $E$ & $\mathrm{TH}$ & 57 & $\mathrm{AL}$ & NA & $C R$ & $\mathrm{CL}$ & $\mathrm{L}$ \\
\hline 12 & $\mathrm{AL}$ & I & $C A$ & $E$ & TH & 58 & $\mathrm{AL}$ & I & ML & $\mathrm{CL}$ & $\mathrm{L}$ \\
\hline 13 & NA & $C R$ & TH & $E$ & TH & 59 & $E$ & I & $\mathrm{TH}$ & TH & $\mathrm{L}$ \\
\hline 14 & NA & $C R$ & $\mathrm{CH}$ & $\mathrm{ML}$ & L & 60 & $\mathrm{AL}$ & NA & $\mathrm{TH}$ & $E$ & $\mathrm{~L}$ \\
\hline 15 & PM & I & $\mathrm{CH}$ & $\mathrm{ML}$ & L & 61 & NA & NA & $T M$ & $\mathrm{CL}$ & $\mathrm{L}$ \\
\hline 16 & $C A$ & $C R$ & NA & I & L & 62 & NA & NA & TH & $\mathrm{TH}$ & $\mathrm{L}$ \\
\hline 17 & $\mathrm{CA}$ & $C R$ & NA & $E$ & $L$ & 63 & $\mathrm{TH}$ & PM & PM & $\mathrm{CL}$ & $\mathrm{L}$ \\
\hline 18 & $C A$ & $C R$ & PM & $E$ & L & 64 & PM & $\mathrm{CM}$ & $\mathrm{CM}$ & $\mathrm{CL}$ & $\mathrm{L}$ \\
\hline 19 & $C A$ & 1 & PM & $E$ & L & 65 & $C A$ & $\mathrm{CM}$ & $\mathrm{TH}$ & TH & $\mathrm{L}$ \\
\hline 20 & $\mathrm{CA}$ & 1 & $\mathrm{CM}$ & $E$ & L & 66 & $C A$ & $\mathrm{CM}$ & $\mathrm{AL}$ & TH & $\mathrm{L}$ \\
\hline 21 & $C A$ & 1 & $\mathrm{AL}$ & $\mathrm{Cl}$ & L & 67 & $C A$ & $\mathrm{AL}$ & $\mathrm{ML}$ & TH & $\mathrm{L}$ \\
\hline 22 & $C A$ & NA & $\mathrm{AL}$ & $\mathrm{CL}$ & TH & 68 & $C A$ & $\mathrm{AL}$ & $\mathrm{AL}$ & ML & $\mathrm{L}$ \\
\hline 23 & $C A$ & $E$ & NA & $E$ & $E$ & 69 & PM & $E$ & $\mathrm{ML}$ & $\mathrm{CL}$ & $\mathrm{L}$ \\
\hline 24 & TM & $E$ & $\mathrm{AL}$ & $\mathrm{L}$ & $\mathrm{TH}$ & 70 & $\mathrm{CA}$ & $\mathrm{AL}$ & $\mathrm{AL}$ & $\mathrm{ML}$ & $\mathrm{L}$ \\
\hline 25 & $E$ & $E$ & NA & $\mathrm{ML}$ & TH & 71 & $C A$ & $E$ & $\mathrm{AL}$ & $\mathrm{CL}$ & $\mathrm{L}$ \\
\hline 26 & PM & 1 & PM & I & TH & 72 & $E$ & $\mathrm{AL}$ & TH & $\mathrm{CL}$ & $\mathrm{L}$ \\
\hline 27 & $\mathrm{CA}$ & $E$ & $C A$ & $\mathrm{CL}$ & $\mathrm{TH}$ & 73 & $E$ & $\mathrm{AL}$ & $\mathrm{TH}$ & $\mathrm{CL}$ & $E$ \\
\hline 28 & $C A$ & $E$ & PM & $\mathrm{CL}$ & $E$ & 74 & L & $\mathrm{AL}$ & NA & TH & $\mathrm{L}$ \\
\hline 29 & PM & $E$ & PM & $\mathrm{CL}$ & TH & 75 & $\mathrm{~L}$ & $\mathrm{AL}$ & NA & $\mathrm{CL}$ & $\mathrm{L}$ \\
\hline 30 & $C A$ & $E$ & $\mathrm{CM}$ & $\mathrm{ML}$ & TH & 76 & $\mathrm{~L}$ & $\mathrm{AL}$ & PM & $\mathrm{CL}$ & $\mathrm{L}$ \\
\hline 31 & $\mathrm{CH}$ & $C R$ & $\mathrm{CM}$ & I & TH & 77 & L & $\mathrm{AL}$ & $C A$ & $\mathrm{CL}$ & $\mathrm{L}$ \\
\hline 32 & $\mathrm{CH}$ & $E$ & $\mathrm{CM}$ & $E$ & TH & 78 & TH & $\mathrm{AL}$ & $\mathrm{CH}$ & TH & $\mathrm{L}$ \\
\hline 33 & $\mathrm{CH}$ & $E$ & $\mathrm{AL}$ & $E$ & TH & 79 & $E$ & $E$ & $\mathrm{TH}$ & TH & $\mathrm{L}$ \\
\hline 34 & PM & $E$ & $\mathrm{AL}$ & $E$ & TH & 80 & $\mathrm{CM}$ & NA & $\mathrm{CM}$ & TH & $\mathrm{L}$ \\
\hline 35 & NA & $\mathrm{ML}$ & $\mathrm{AL}$ & $\mathrm{ML}$ & TH & 81 & $\mathrm{CM}$ & NA & $\mathrm{CM}$ & $\mathrm{CL}$ & $\mathrm{L}$ \\
\hline 36 & $\mathrm{CM}$ & $\mathrm{ML}$ & $\mathrm{AL}$ & $\mathrm{CL}$ & TH & 82 & $\mathrm{CM}$ & NA & $\mathrm{AL}$ & $\mathrm{CL}$ & $\mathrm{L}$ \\
\hline 37 & $\mathrm{CM}$ & $\mathrm{ML}$ & NA & $\mathrm{CL}$ & $\mathrm{TH}$ & 83 & $\mathrm{CM}$ & $E$ & $\mathrm{AL}$ & $\mathrm{CL}$ & $\mathrm{L}$ \\
\hline 38 & $\mathrm{AL}$ & L & NA & $\mathrm{CL}$ & L & 84 & NA & $C A$ & $\mathrm{AL}$ & $\mathrm{CL}$ & $\mathrm{L}$ \\
\hline 39 & NA & L & PM & TH & $L$ & 85 & $\mathrm{AL}$ & TH & $\mathrm{AL}$ & TH & $\mathrm{L}$ \\
\hline 40 & NA & $E$ & PM & $\mathrm{CL}$ & L & 86 & $\mathrm{AL}$ & L & $\mathrm{CA}$ & $\mathrm{TH}$ & $\mathrm{L}$ \\
\hline 41 & $\mathrm{AL}$ & $\mathrm{CM}$ & $\mathrm{CA}$ & $\mathrm{ML}$ & L & 87 & NA & L & $\mathrm{TH}$ & $\mathrm{CL}$ & $\mathrm{L}$ \\
\hline 42 & $E$ & $\mathrm{CM}$ & $C A$ & $\mathrm{CL}$ & L & 88 & NA & CA & $E$ & TH & $\mathrm{TH}$ \\
\hline 43 & $\mathrm{AL}$ & $\mathrm{AL}$ & $L$ & $\mathrm{Cl}$ & $L$ & 89 & $\mathrm{AL}$ & $C A$ & MI & $\mathrm{TH}$ & $\mathrm{TH}$ \\
\hline 44 & $\mathrm{AL}$ & $\mathrm{AL}$ & $L$ & $\mathrm{CL}$ & $L$ & 90 & NA & $C A$ & PM & $\mathrm{CL}$ & $\mathrm{TH}$ \\
\hline 45 & NA & $\mathrm{AL}$ & MI & $\mathrm{Cl}$ & L & & & & & & \\
\hline
\end{tabular}

Fuente: Elaboración Propia. 
Figura No 67: Distribución de Trabajo de Asentado de Ladrillo - Carta Balance ${ }^{\circ} 8$

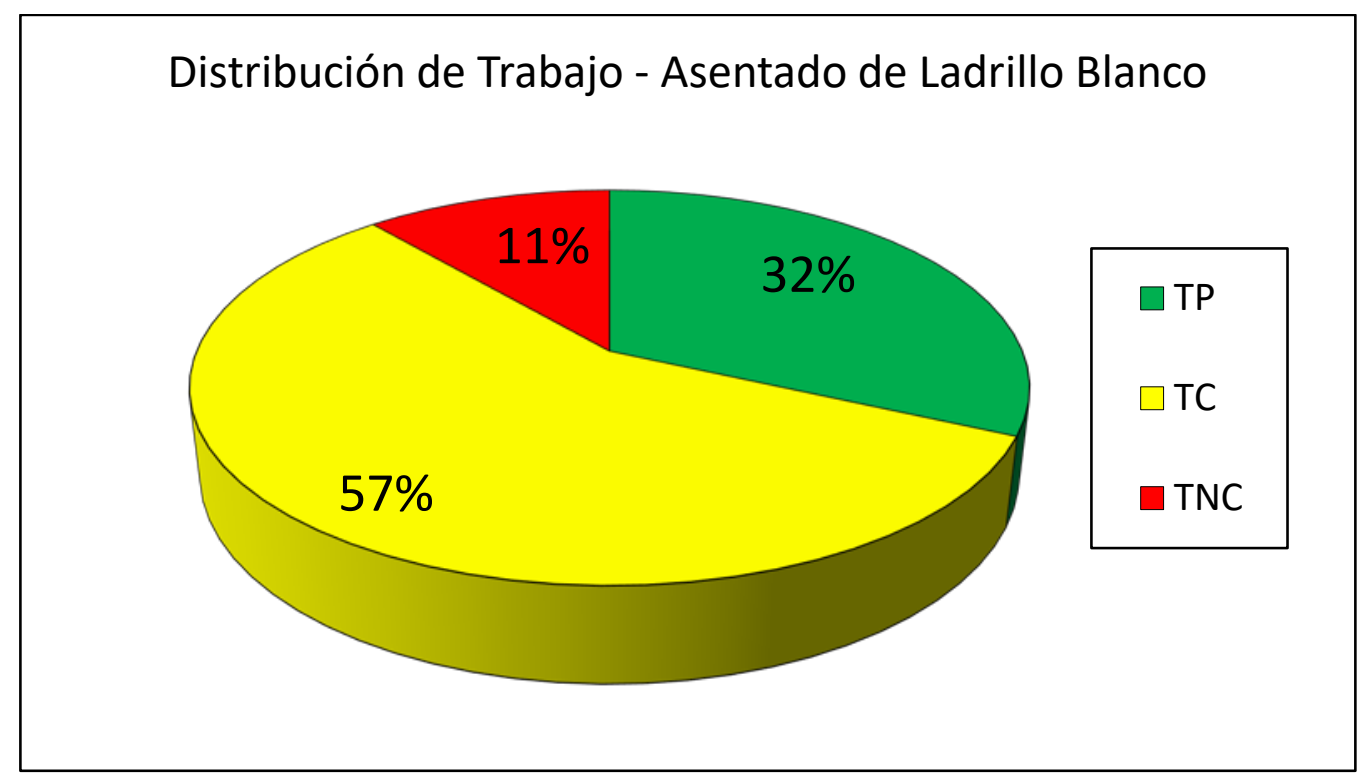

Fuente: Elaboración Propia.

Figura $N^{\circ}$ 68: Distribución de Trabajo Productivo - Carta Balance ${ }^{\circ} 8$

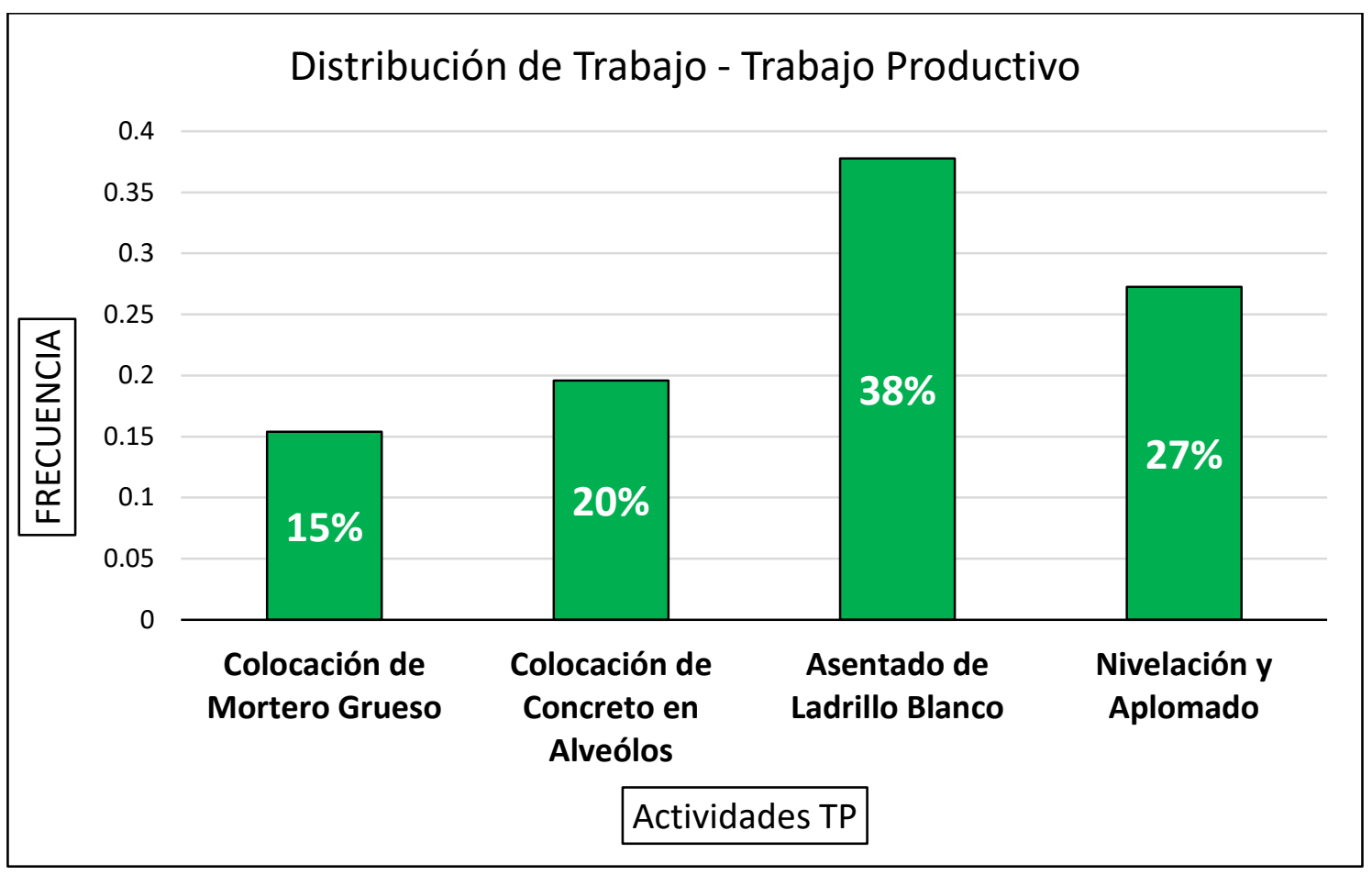

Fuente: Elaboración Propia. 
Figura $N^{\circ}$ 69: Distribución de Trabajo Contributorio - Carta Balance ${ }^{\circ} 8$

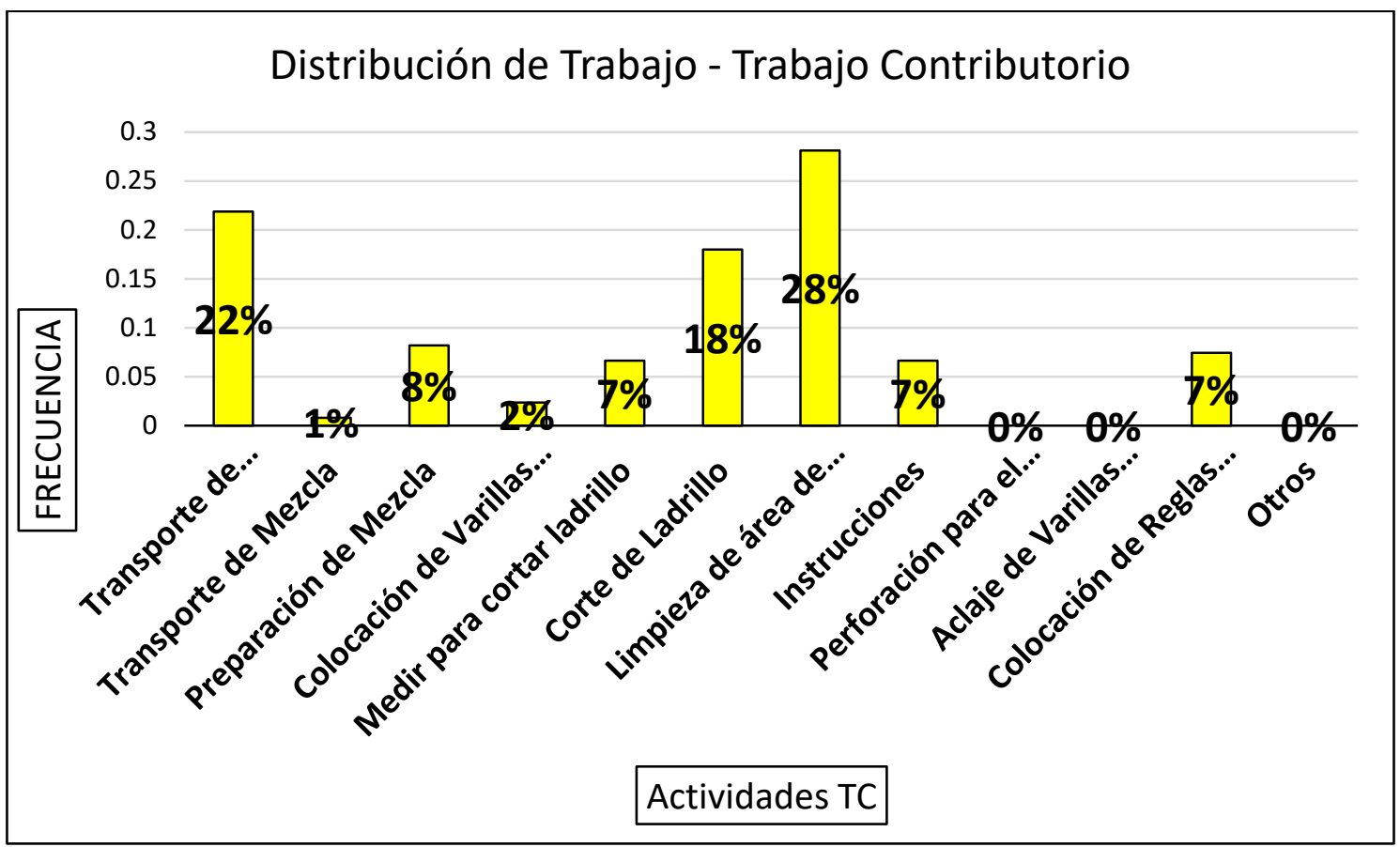

Fuente: Elaboración Propia.

Figura $N^{\circ} 70$ : Distribución de Trabajo No Contributorio - Carta Balance ${ }^{\circ} 8$

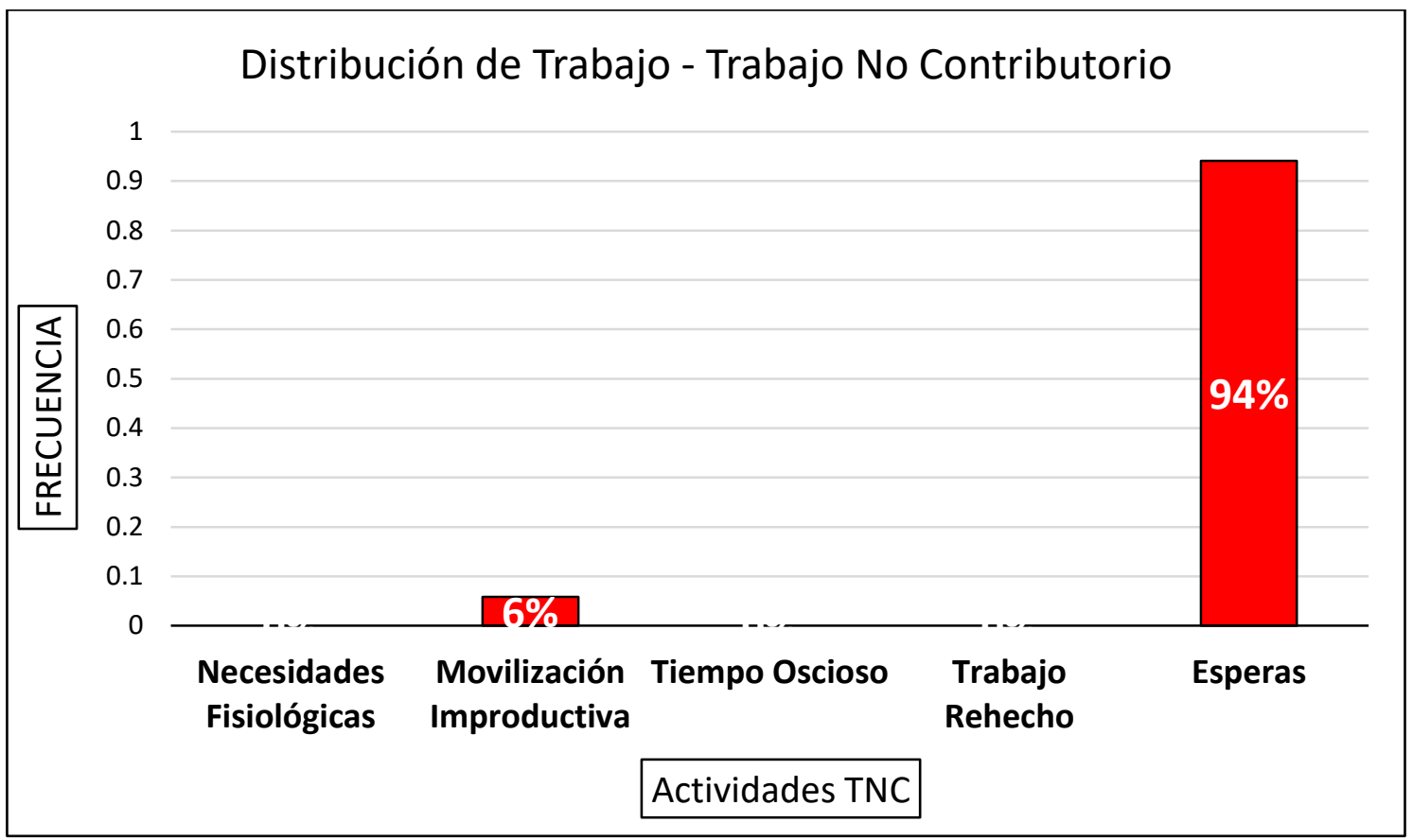

Fuente: Elaboración Propia. 
Tabla No 21: Carta Balance ${ }^{\circ} 22$ - Asentado de Ladrillo

\begin{tabular}{|c|c|c|c|c|c|c|c|c|c|c|c|}
\hline $\mathrm{N}^{0}$ & Obrero \#1 & Obrero\#2 & Obrero \#3 & Obrero \#4 & Obrero \#5 & 46 & $\mathrm{AL}$ & $\mathrm{NF}$ & NA & $\mathrm{CL}$ & TH \\
\hline 1 & $\mathrm{AL}$ & 1 & 1 & $\mathrm{CL}$ & TO & 47 & $\mathrm{CM}$ & $\mathrm{NF}$ & NA & $\mathrm{CL}$ & $\mathrm{TH}$ \\
\hline 2 & $\mathrm{AL}$ & 1 & I & $\mathrm{CL}$ & TO & 48 & $\mathrm{AL}$ & $\mathrm{NF}$ & $\mathrm{CA}$ & $\mathrm{CL}$ & $\mathrm{TH}$ \\
\hline 3 & $\mathrm{AL}$ & $\mathrm{AL}$ & $C A$ & $\mathrm{ML}$ & TO & 49 & $\mathrm{AL}$ & $\mathrm{NF}$ & $C A$ & $\mathrm{ML}$ & $\mathrm{L}$ \\
\hline 4 & $\mathrm{CM}$ & $\mathrm{AL}$ & $C A$ & $\mathrm{TH}$ & TO & 50 & NA & $\mathrm{CM}$ & $\mathrm{CA}$ & $\mathrm{CL}$ & $\mathrm{L}$ \\
\hline 5 & $\mathrm{AL}$ & $\mathrm{AL}$ & $\mathrm{L}$ & TO & TO & 51 & NA & $\mathrm{CM}$ & $\mathrm{CA}$ & ML & $\mathrm{L}$ \\
\hline 6 & $\mathrm{AL}$ & $\mathrm{AL}$ & $\mathrm{L}$ & ML & TO & 52 & $\mathrm{TM}$ & $\mathrm{AL}$ & $\mathrm{CA}$ & $\mathrm{CL}$ & $\mathrm{L}$ \\
\hline 7 & $\mathrm{AL}$ & $\mathrm{AL}$ & $\mathrm{L}$ & $\mathrm{CL}$ & TO & 53 & $\mathrm{TH}$ & $\mathrm{AL}$ & $\mathrm{CA}$ & $\mathrm{TH}$ & $\mathrm{L}$ \\
\hline 8 & $\mathrm{AL}$ & $\mathrm{AL}$ & $L$ & $\mathrm{CL}$ & TO & 54 & $\mathrm{PM}$ & $\mathrm{AL}$ & $C A$ & $\mathrm{CL}$ & $\mathrm{L}$ \\
\hline 9 & NA & $\mathrm{AL}$ & TO & $\mathrm{CL}$ & TO & 55 & $\mathrm{CA}$ & $E$ & $\mathrm{CM}$ & $\mathrm{CL}$ & $\mathrm{L}$ \\
\hline 10 & NA & NA & TO & $\mathrm{CL}$ & TO & 56 & $C A$ & $\mathrm{AL}$ & $\mathrm{CM}$ & $\mathrm{ML}$ & $\mathrm{TH}$ \\
\hline 11 & NA & NA & TO & $\mathrm{CL}$ & TO & 57 & $C A$ & NA & $\mathrm{AL}$ & $\mathrm{ML}$ & TH \\
\hline 12 & $\mathrm{TM}$ & TO & TO & TO & TO & 58 & PM & $\mathrm{AL}$ & $\mathrm{NA}$ & $\mathrm{CL}$ & $\mathrm{TH}$ \\
\hline 13 & $\mathrm{TM}$ & TO & TO & TO & TO & 59 & $\mathrm{PM}$ & NA & $\mathrm{AL}$ & $\mathrm{CL}$ & $\mathrm{TH}$ \\
\hline 14 & TO & TO & TO & TO & TO & 60 & $\mathrm{CM}$ & $\mathrm{PM}$ & $\mathrm{AL}$ & $\mathrm{TH}$ & TH \\
\hline 15 & TO & TO & TO & TO & TO & 61 & $\mathrm{CM}$ & NA & NA & $\mathrm{CL}$ & $\mathrm{TH}$ \\
\hline 16 & TO & TO & TO & TO & TO & 62 & $\mathrm{CM}$ & NA & $\mathrm{AL}$ & $\mathrm{CL}$ & $\mathrm{L}$ \\
\hline 17 & TO & TO & TO & TO & TO & 63 & $\mathrm{CM}$ & NA & $\mathrm{AL}$ & $\mathrm{CL}$ & $\mathrm{L}$ \\
\hline 18 & TO & TO & TO & TO & TO & 64 & $\mathrm{AL}$ & $\mathrm{PM}$ & $\mathrm{AL}$ & $\mathrm{CL}$ & $\mathrm{L}$ \\
\hline 19 & TO & TO & TO & TO & TO & 65 & $\mathrm{AL}$ & $\mathrm{AL}$ & $\mathrm{AL}$ & $\mathrm{CL}$ & $\mathrm{L}$ \\
\hline 20 & TO & TO & TO & TO & TO & 66 & $\mathrm{AL}$ & $\mathrm{AL}$ & $\mathrm{AL}$ & $\mathrm{CL}$ & $\mathrm{L}$ \\
\hline 21 & TO & TO & TO & TO & TO & 67 & $\mathrm{AL}$ & $\mathrm{AL}$ & $\mathrm{AL}$ & $\mathrm{CL}$ & $\mathrm{L}$ \\
\hline 22 & TO & TO & TO & TO & TO & 68 & $\mathrm{AL}$ & $\mathrm{AL}$ & NA & $\mathrm{TH}$ & $\mathrm{L}$ \\
\hline 23 & TO & TO & TO & TO & TO & 69 & $\mathrm{AL}$ & $\mathrm{CM}$ & NA & $\mathrm{ML}$ & $\mathrm{L}$ \\
\hline 24 & $C A$ & $\mathrm{CM}$ & $L$ & $\mathrm{TH}$ & $\mathrm{L}$ & 70 & $\mathrm{AL}$ & $\mathrm{CM}$ & NA & $\mathrm{Cl}$ & $\mathrm{L}$ \\
\hline 25 & $C A$ & $\mathrm{AL}$ & $\mathrm{L}$ & $\mathrm{CL}$ & L & 71 & $\mathrm{AL}$ & $\mathrm{AL}$ & NA & $\mathrm{CL}$ & $\mathrm{TH}$ \\
\hline 26 & AV & $\mathrm{AL}$ & $\mathrm{CH}$ & $\mathrm{TH}$ & $\mathrm{L}$ & 72 & $\mathrm{AL}$ & $\mathrm{AL}$ & NA & $\mathrm{CL}$ & $\mathrm{CL}$ \\
\hline 27 & $\mathrm{AV}$ & $\mathrm{AL}$ & $\mathrm{CH}$ & $\mathrm{ML}$ & $\mathrm{L}$ & 73 & $\mathrm{AL}$ & $\mathrm{AL}$ & NA & $\mathrm{CL}$ & $\mathrm{CL}$ \\
\hline 28 & AV & NA & $\mathrm{CM}$ & $\mathrm{CL}$ & $\mathrm{L}$ & 74 & $\mathrm{AL}$ & NA & NA & $\mathrm{CL}$ & $\mathrm{CL}$ \\
\hline 29 & AV & NA & NA & $\mathrm{CL}$ & $\mathrm{L}$ & 75 & NA & NA & $\mathrm{CA}$ & $\mathrm{ML}$ & $\mathrm{CL}$ \\
\hline 30 & $\mathrm{CA}$ & PM & $\mathrm{AL}$ & $\mathrm{CL}$ & $\mathrm{L}$ & 76 & NA & $\mathrm{TM}$ & $\mathrm{CA}$ & $\mathrm{CL}$ & $\mathrm{CL}$ \\
\hline 31 & $C A$ & $C A$ & NA & $\mathrm{CL}$ & $\mathrm{L}$ & 77 & NA & TH & $\mathrm{CA}$ & TH & $\mathrm{CL}$ \\
\hline 32 & $\mathrm{CH}$ & $C A$ & NA & $\mathrm{ML}$ & $\mathrm{L}$ & 78 & $\mathrm{CA}$ & $\mathrm{CA}$ & $\mathrm{CA}$ & $\mathrm{CL}$ & $\mathrm{CL}$ \\
\hline 33 & $\mathrm{CH}$ & $C A$ & $\mathrm{AL}$ & $\mathrm{CL}$ & $L$ & 79 & $\mathrm{CA}$ & $\mathrm{CA}$ & $\mathrm{CA}$ & $\mathrm{CL}$ & $\mathrm{CL}$ \\
\hline 34 & $\mathrm{CM}$ & $C A$ & $\mathrm{AL}$ & $\mathrm{CL}$ & $\mathrm{TH}$ & 80 & $\mathrm{CA}$ & $\mathrm{CA}$ & $\mathrm{CA}$ & $\mathrm{Cl}$ & $\mathrm{TH}$ \\
\hline 35 & $\mathrm{CM}$ & $\mathrm{CA}$ & $\mathrm{AL}$ & ML & $\mathrm{TH}$ & 81 & $\mathrm{CA}$ & $\mathrm{CA}$ & $\mathrm{L}$ & $\mathrm{CL}$ & $\mathrm{TH}$ \\
\hline 36 & $\mathrm{CM}$ & $C A$ & $\mathrm{AL}$ & $\mathrm{CL}$ & $\mathrm{TH}$ & 82 & $C A$ & $\mathrm{~L}$ & $\mathrm{~L}$ & $\mathrm{ML}$ & $\mathrm{TH}$ \\
\hline 37 & PM & $C A$ & $\mathrm{AL}$ & $\mathrm{CL}$ & $\mathrm{TH}$ & 83 & $\mathrm{CA}$ & $\mathrm{CA}$ & $L$ & $\mathrm{ML}$ & $\mathrm{TH}$ \\
\hline 38 & $\mathrm{AL}$ & $C A$ & NA & $\mathrm{CL}$ & $\mathrm{TH}$ & 84 & $C A$ & $\mathrm{CA}$ & $\mathrm{L}$ & $E$ & TH \\
\hline 39 & $\mathrm{AL}$ & NA & NA & $\mathrm{ML}$ & $\mathrm{TH}$ & 85 & $\mathrm{~L}$ & $\mathrm{CA}$ & $\mathrm{L}$ & $\mathrm{L}$ & $\mathrm{L}$ \\
\hline 40 & $\mathrm{AL}$ & PM & NA & $\mathrm{CL}$ & $\mathrm{TH}$ & 86 & $\mathrm{~L}$ & $\mathrm{CA}$ & $\mathrm{L}$ & $\mathrm{CL}$ & $\mathrm{L}$ \\
\hline 41 & $\mathrm{AL}$ & $\mathrm{NF}$ & NA & $\mathrm{CL}$ & $\mathrm{TH}$ & 87 & $\mathrm{~L}$ & $\mathrm{CA}$ & NA & $\mathrm{CL}$ & $\mathrm{L}$ \\
\hline 42 & $\mathrm{AL}$ & $\mathrm{NF}$ & NA & $\mathrm{CL}$ & $\mathrm{TH}$ & 88 & $\mathrm{~L}$ & $\mathrm{~L}$ & NA & $\mathrm{ML}$ & $\mathrm{L}$ \\
\hline 43 & $\mathrm{AL}$ & $\mathrm{NF}$ & NA & $\mathrm{CL}$ & $\mathrm{TH}$ & 89 & $\mathrm{~L}$ & $\mathrm{~L}$ & NA & $\mathrm{TH}$ & $\mathrm{L}$ \\
\hline 44 & $\mathrm{AL}$ & $\mathrm{NF}$ & NA & $\mathrm{ML}$ & $\mathrm{TH}$ & 90 & $\mathrm{PM}$ & $\mathrm{L}$ & $\mathrm{NA}$ & $\mathrm{CL}$ & $\mathrm{L}$ \\
\hline 45 & $\mathrm{AL}$ & $\mathrm{NF}$ & NA & $\mathrm{ML}$ & $\mathrm{TH}$ & & & & & & \\
\hline
\end{tabular}

Fuente: Elaboración Propia. 
Figura N 71: Distribución de Trabajo de Asentado de Ladrillo - Carta Balance ${ }^{\circ} 22$

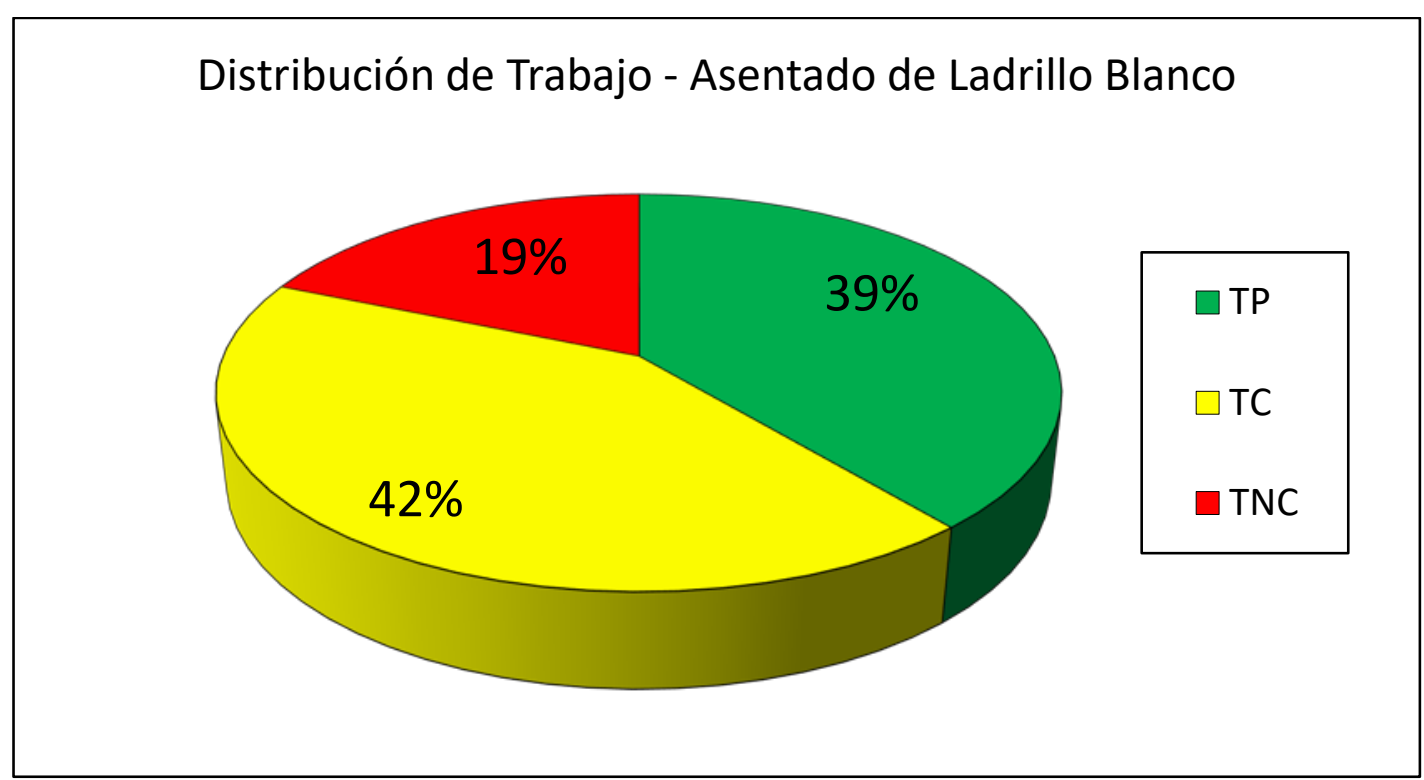

Fuente: Elaboración Propia.

Figura $N^{\circ} 72$ : Distribución de Trabajo Productivo - Carta Balance ${ }^{\circ} 22$

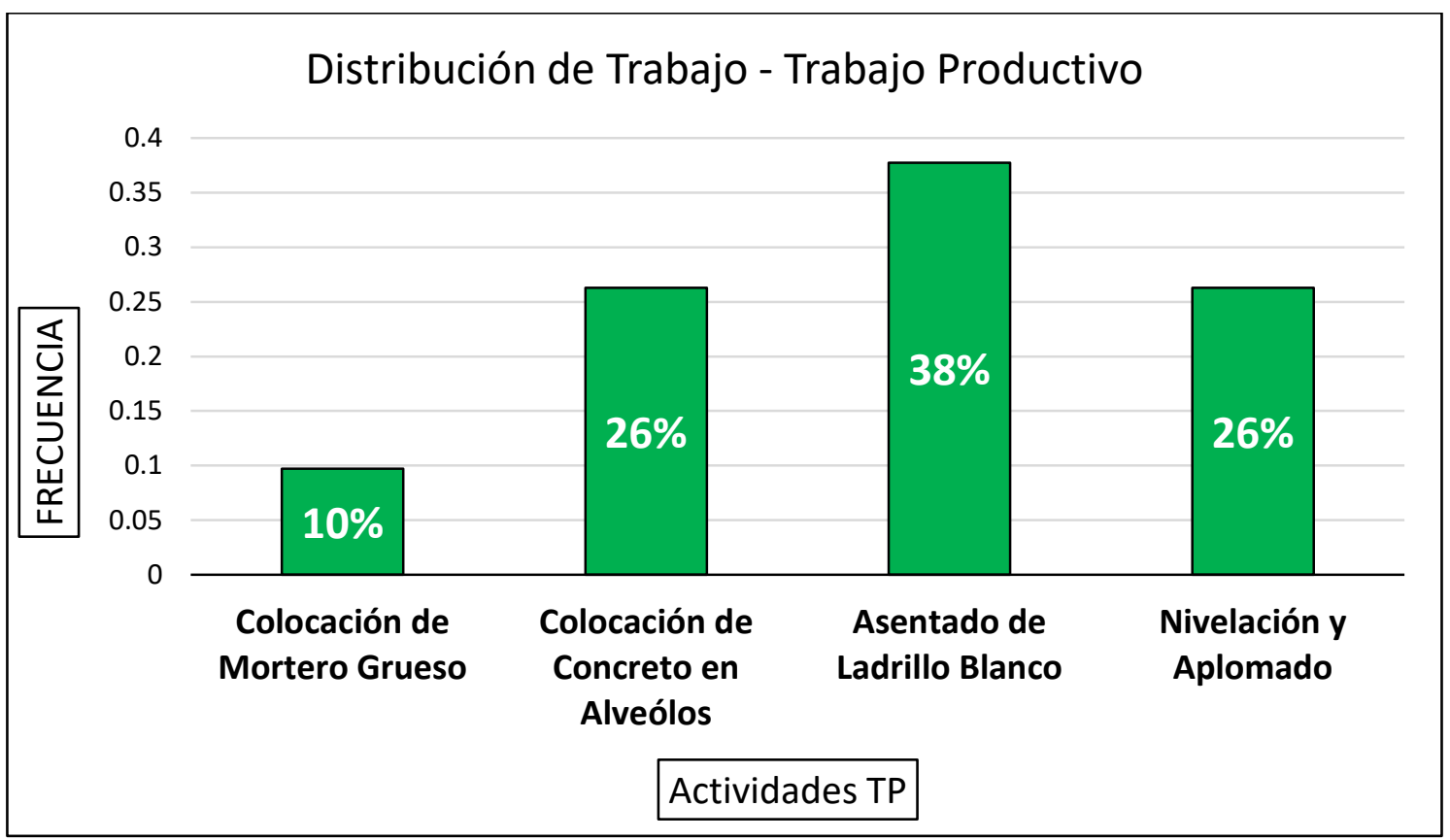

Fuente: Elaboración Propia. 
Figura Nº 73: Distribución de Trabajo Contributorio - Carta Balance ${ }^{\circ} 22$

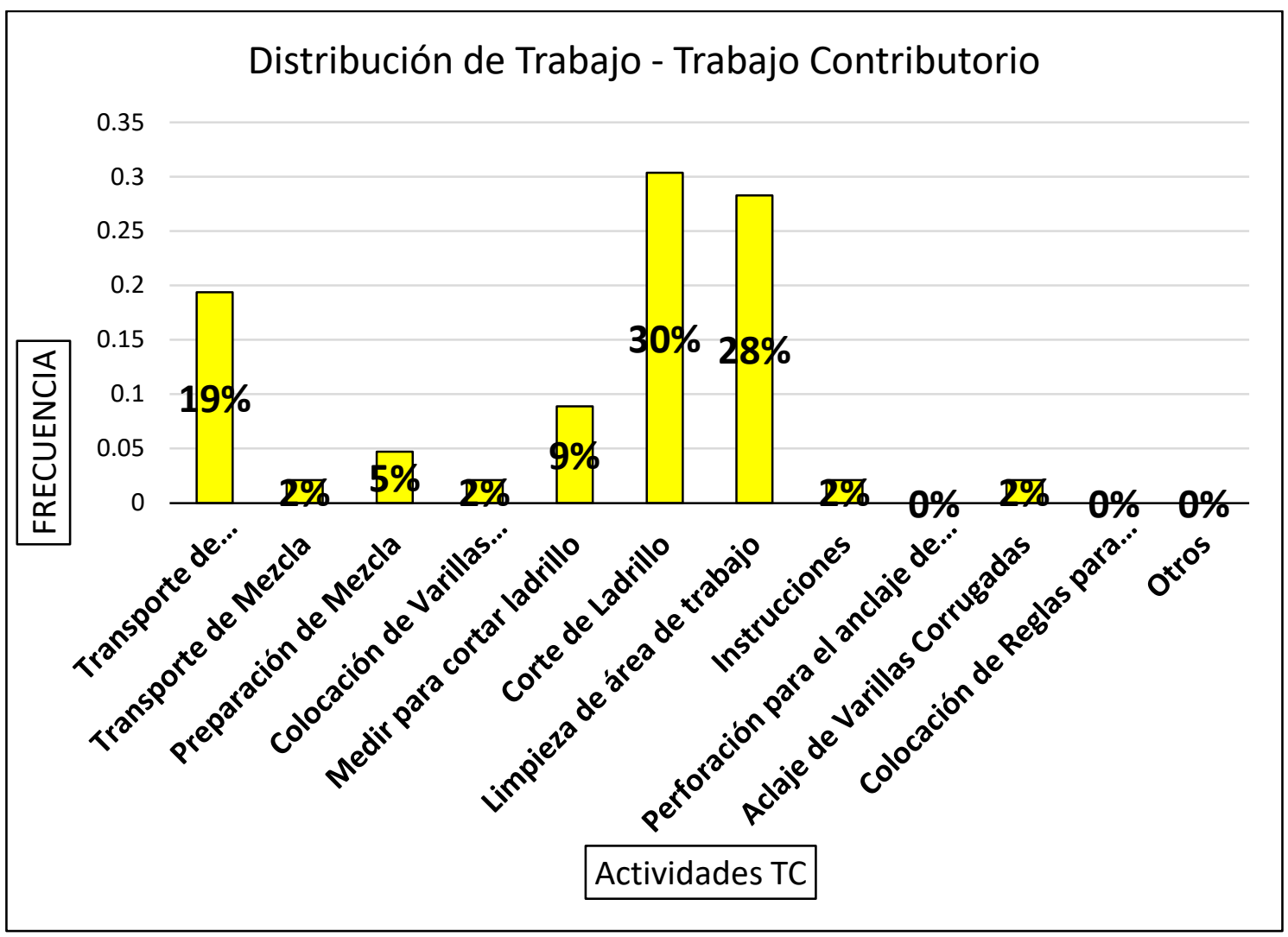

Fuente: Elaboración Propia.

Figura $N^{\circ} 74$ : Distribución de Trabajo No Contributorio - Carta Balance ${ }^{\circ} 22$

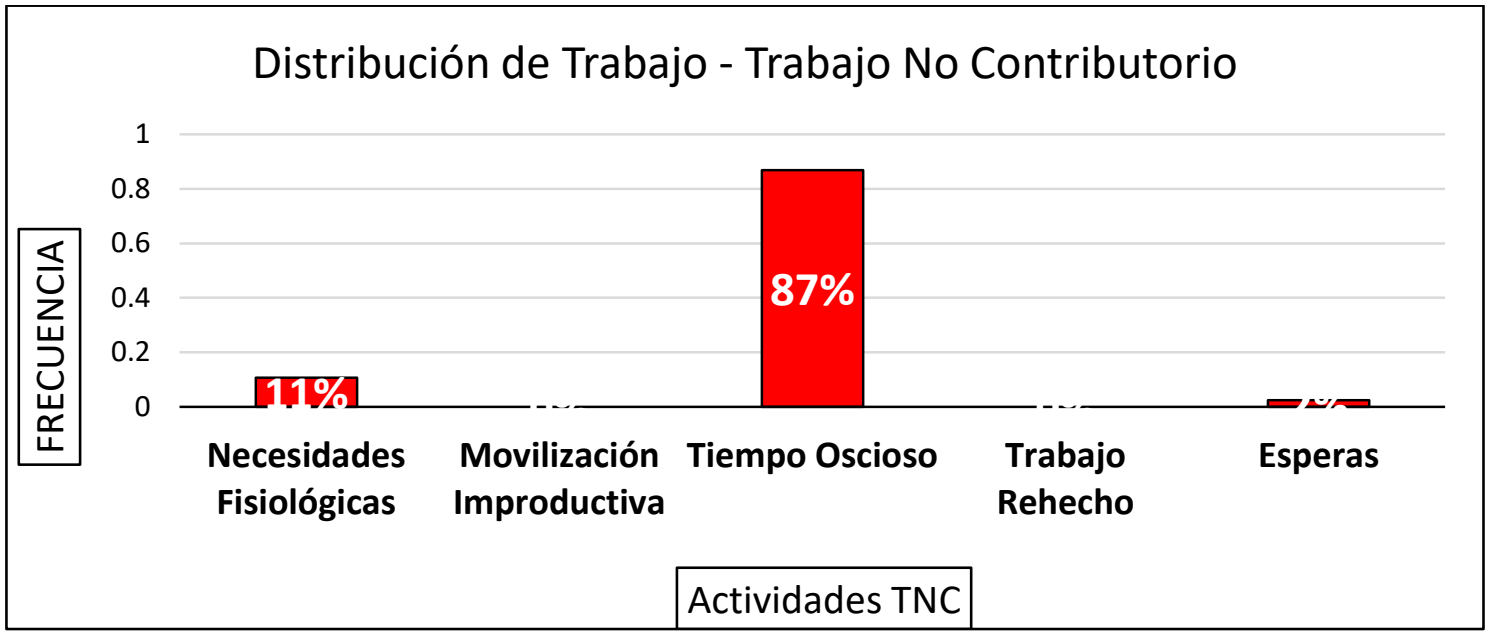

Fuente: Elaboración Propia. 
Tabla No 22: Carta Balance ${ }^{\circ} 23$ - Asentado de Ladrillo

\begin{tabular}{|c|c|c|c|c|c|c|c|c|c|c|c|}
\hline $\mathrm{N}^{0}$ & Obrero\#1 & Obrero\#2 & Obrero \#3 & Obrero \#4 & Obrero\#5 & 46 & $E$ & $\mathrm{TH}$ & NA & $\mathrm{ML}$ & TH \\
\hline 1 & $C A$ & L & $\mathrm{AL}$ & $\mathrm{CL}$ & $E$ & 47 & PM & $\mathrm{TH}$ & NA & $\mathrm{ML}$ & $\mathrm{TH}$ \\
\hline 2 & $\mathrm{CA}$ & $\mathrm{L}$ & $\mathrm{AL}$ & $\mathrm{CL}$ & $E$ & 48 & $\mathrm{CA}$ & $\mathrm{TH}$ & $\mathrm{AL}$ & $\mathrm{ML}$ & $\mathrm{TH}$ \\
\hline 3 & $C A$ & $\mathrm{~L}$ & NA & $\mathrm{TH}$ & $E$ & 49 & $\mathrm{CM}$ & $\mathrm{L}$ & NA & $E$ & $\mathrm{TH}$ \\
\hline 4 & $C A$ & $\mathrm{~L}$ & NA & $M L$ & $E$ & 50 & PM & $\mathrm{L}$ & PM & $\mathrm{ML}$ & $\mathrm{TH}$ \\
\hline 5 & $C A$ & $\mathrm{~L}$ & NA & $\mathrm{CL}$ & $E$ & 51 & $E$ & $\mathrm{PM}$ & $C A$ & $E$ & $\mathrm{TH}$ \\
\hline 6 & $C A$ & $\mathrm{~L}$ & NA & $\mathrm{ML}$ & $E$ & 52 & $C R$ & PM & $C A$ & $\mathrm{ML}$ & $\mathrm{TH}$ \\
\hline 7 & $C A$ & TM & $\mathrm{CA}$ & $\mathrm{CL}$ & $E$ & 53 & $C R$ & $\mathrm{CM}$ & $C A$ & $\mathrm{ML}$ & $\mathrm{TH}$ \\
\hline 8 & $\mathrm{~L}$ & $\mathrm{~L}$ & $C A$ & $\mathrm{~L}$ & $E$ & 54 & $\mathrm{AL}$ & $\mathrm{CM}$ & PM & $\mathrm{TH}$ & $\mathrm{TH}$ \\
\hline 9 & $L$ & $\mathrm{~L}$ & $\mathrm{AL}$ & $\mathrm{CL}$ & $E$ & 55 & $\mathrm{TH}$ & $\mathrm{CM}$ & $\mathrm{NF}$ & $\mathrm{CL}$ & $\mathrm{TH}$ \\
\hline 10 & TM & $\mathrm{TH}$ & L & L & $E$ & 56 & $C R$ & I & $\mathrm{NF}$ & ML & $\mathrm{TH}$ \\
\hline 11 & $\mathrm{TM}$ & $\mathrm{TM}$ & $\mathrm{TH}$ & $L$ & $E$ & 57 & $C R$ & 1 & $\mathrm{NF}$ & $\mathrm{CL}$ & $\mathrm{TH}$ \\
\hline 12 & $E$ & $\mathrm{TH}$ & $\mathrm{TH}$ & $L$ & $E$ & 58 & $C R$ & 1 & $\mathrm{NF}$ & $\mathrm{CL}$ & $\mathrm{TH}$ \\
\hline 13 & $L$ & $\mathrm{~L}$ & PM & $\mathrm{L}$ & $E$ & 59 & $\mathrm{AL}$ & $\mathrm{AL}$ & $\mathrm{NF}$ & $\mathrm{CL}$ & $\mathrm{L}$ \\
\hline 14 & $\mathrm{~L}$ & $C R$ & PM & $\mathrm{L}$ & $E$ & 60 & $\mathrm{CA}$ & $\mathrm{AL}$ & $\mathrm{NF}$ & $\mathrm{CL}$ & $\mathrm{L}$ \\
\hline 15 & $L$ & $C R$ & PM & $\mathrm{CL}$ & $E$ & 61 & $\mathrm{AV}$ & 1 & $C A$ & $\mathrm{CL}$ & $\mathrm{L}$ \\
\hline 16 & L & $C R$ & $\mathrm{TH}$ & $\mathrm{ML}$ & $E$ & 62 & $\mathrm{CH}$ & 1 & CM & $\mathrm{ML}$ & L \\
\hline 17 & $L$ & $C R$ & $\mathrm{CH}$ & $\mathrm{ML}$ & $E$ & 63 & $\mathrm{TM}$ & 1 & $\mathrm{CM}$ & $\mathrm{ML}$ & $\mathrm{TH}$ \\
\hline 18 & NA & $C R$ & $\mathrm{CM}$ & $\mathrm{CL}$ & $E$ & 64 & $\mathrm{AL}$ & $\mathrm{AL}$ & $\mathrm{AL}$ & $\mathrm{CL}$ & $\mathrm{TH}$ \\
\hline 19 & NA & $C R$ & $\mathrm{AL}$ & $\mathrm{E}$ & $E$ & 65 & $\mathrm{AL}$ & $\mathrm{AL}$ & $\mathrm{AL}$ & $\mathrm{TH}$ & $\mathrm{TH}$ \\
\hline 20 & NA & $C R$ & NA & $\mathrm{E}$ & $E$ & 66 & $\mathrm{CH}$ & $\mathrm{AL}$ & $\mathrm{AL}$ & $\mathrm{TH}$ & $\mathrm{TH}$ \\
\hline 21 & 1 & 1 & $\mathrm{CM}$ & $E$ & $E$ & 67 & $\mathrm{CM}$ & $\mathrm{AL}$ & $\mathrm{AL}$ & $\mathrm{CL}$ & $\mathrm{TH}$ \\
\hline 22 & NA & $C R$ & $\mathrm{AL}$ & $E$ & $\mathrm{TH}$ & 68 & $\mathrm{AL}$ & $\mathrm{AL}$ & $\mathrm{AL}$ & $E$ & $\mathrm{TH}$ \\
\hline 23 & $\mathrm{TH}$ & $C R$ & $\mathrm{AL}$ & $E$ & $\mathrm{TH}$ & 69 & $\mathrm{AL}$ & NA & $\mathrm{AL}$ & $\mathrm{CL}$ & $\mathrm{TH}$ \\
\hline 24 & $\mathrm{TH}$ & $C R$ & $\mathrm{AL}$ & $E$ & $\mathrm{TH}$ & 70 & $\mathrm{CA}$ & NA & $\mathrm{AL}$ & $\mathrm{TH}$ & $\mathrm{TH}$ \\
\hline 25 & $C R$ & $C R$ & $\mathrm{AL}$ & $E$ & $\mathrm{TH}$ & 71 & $\mathrm{TH}$ & NA & $\mathrm{AL}$ & $\mathrm{ML}$ & $\mathrm{TH}$ \\
\hline 26 & $C R$ & $C R$ & NA & $E$ & $\mathrm{TH}$ & 72 & $\mathrm{CM}$ & NA & NA & $\mathrm{CL}$ & $\mathrm{TH}$ \\
\hline 27 & $L$ & $C R$ & $\mathrm{AL}$ & $E$ & $\mathrm{TH}$ & 73 & NA & $\mathrm{PM}$ & NA & $\mathrm{ML}$ & $\mathrm{TH}$ \\
\hline 28 & 1 & $C R$ & NA & $E$ & $\mathrm{TH}$ & 74 & NA & $\mathrm{CA}$ & NA & $\mathrm{ML}$ & $\mathrm{TH}$ \\
\hline 29 & $\mathrm{~L}$ & $C R$ & NA & $\mathrm{E}$ & L & 75 & AV & $\mathrm{CA}$ & $C A$ & $\mathrm{CL}$ & $\mathrm{TH}$ \\
\hline 30 & $\mathrm{~L}$ & $C R$ & NA & $E$ & $\mathrm{~L}$ & 76 & $\mathrm{AV}$ & $\mathrm{CA}$ & $C A$ & $\mathrm{CL}$ & $\mathrm{TH}$ \\
\hline 31 & $\mathrm{~L}$ & $C R$ & NA & $E$ & $\mathrm{TH}$ & 77 & $\mathrm{CA}$ & $\mathrm{CA}$ & $C A$ & $\mathrm{CL}$ & $\mathrm{TH}$ \\
\hline 32 & $L$ & $C R$ & NA & $\mathrm{E}$ & $\mathrm{CL}$ & 78 & $\mathrm{PM}$ & $\mathrm{AV}$ & $\mathrm{TH}$ & $\mathrm{ML}$ & $\mathrm{L}$ \\
\hline 33 & $\mathrm{~L}$ & $C R$ & NA & $E$ & $\mathrm{CL}$ & 79 & $\mathrm{CM}$ & CA & $\mathrm{AV}$ & $\mathrm{ML}$ & $\mathrm{L}$ \\
\hline 34 & $\mathrm{CH}$ & $C R$ & $C A$ & $\mathrm{CL}$ & $\mathrm{CL}$ & 80 & $\mathrm{AL}$ & $\mathrm{PM}$ & $\mathrm{AV}$ & $\mathrm{CL}$ & L \\
\hline 35 & MI & $C R$ & $C A$ & $\mathrm{ML}$ & $\mathrm{CL}$ & 81 & $\mathrm{AL}$ & $\mathrm{CM}$ & $\mathrm{AV}$ & $\mathrm{CL}$ & $\mathrm{L}$ \\
\hline 36 & $\mathrm{AL}$ & $C R$ & $C A$ & $\mathrm{TH}$ & $\mathrm{TH}$ & 82 & $\mathrm{CA}$ & $\mathrm{CM}$ & $\mathrm{CM}$ & $\mathrm{Cl}$ & $\mathrm{TH}$ \\
\hline 37 & NA & $C R$ & PM & $\mathrm{CL}$ & $\mathrm{TH}$ & 83 & $\mathrm{CH}$ & $\mathrm{AL}$ & $\mathrm{CM}$ & $\mathrm{CL}$ & $\mathrm{TH}$ \\
\hline 38 & $\mathrm{AL}$ & $C R$ & $\mathrm{CM}$ & $\mathrm{ML}$ & $\mathrm{TH}$ & 84 & $\mathrm{CM}$ & $\mathrm{AL}$ & $\mathrm{CM}$ & $\mathrm{CL}$ & $\mathrm{TH}$ \\
\hline 39 & NA & $C R$ & $\mathrm{CM}$ & $\mathrm{ML}$ & $\mathrm{TH}$ & 85 & $\mathrm{CH}$ & $\mathrm{AL}$ & $\mathrm{AL}$ & $\mathrm{CL}$ & $\mathrm{TH}$ \\
\hline 40 & 1 & 1 & $\mathrm{CM}$ & $\mathrm{CL}$ & $\mathrm{TH}$ & 86 & $\mathrm{AL}$ & $\mathrm{AL}$ & $\mathrm{AL}$ & $\mathrm{CL}$ & $\mathrm{TH}$ \\
\hline 41 & 1 & 1 & $\mathrm{AL}$ & $\mathrm{CL}$ & $\mathrm{TH}$ & 87 & NA & $\mathrm{AL}$ & $\mathrm{AL}$ & $\mathrm{ML}$ & $\mathrm{TH}$ \\
\hline 42 & 1 & 1 & $\mathrm{AL}$ & $\mathrm{CL}$ & TH & 88 & NA & $\mathrm{CH}$ & NA & $\mathrm{ML}$ & $\mathrm{TH}$ \\
\hline 43 & NA & $C R$ & $\mathrm{AL}$ & $\mathrm{CL}$ & $\mathrm{TH}$ & 89 & $C A$ & $\mathrm{AL}$ & $\mathrm{AL}$ & $\mathrm{CL}$ & $\mathrm{TH}$ \\
\hline 44 & NA & $C R$ & $\mathrm{TH}$ & $\mathrm{ML}$ & $L$ & 90 & PM & $\mathrm{AL}$ & NA & $\mathrm{CL}$ & $\mathrm{TH}$ \\
\hline 45 & NA & $C R$ & NA & L & $\mathrm{TH}$ & & & & & & \\
\hline
\end{tabular}

Fuente: Elaboración Propia. 
Figura N 75: Distribución de Trabajo de Asentado de Ladrillo - Carta Balance ${ }^{\circ} 23$

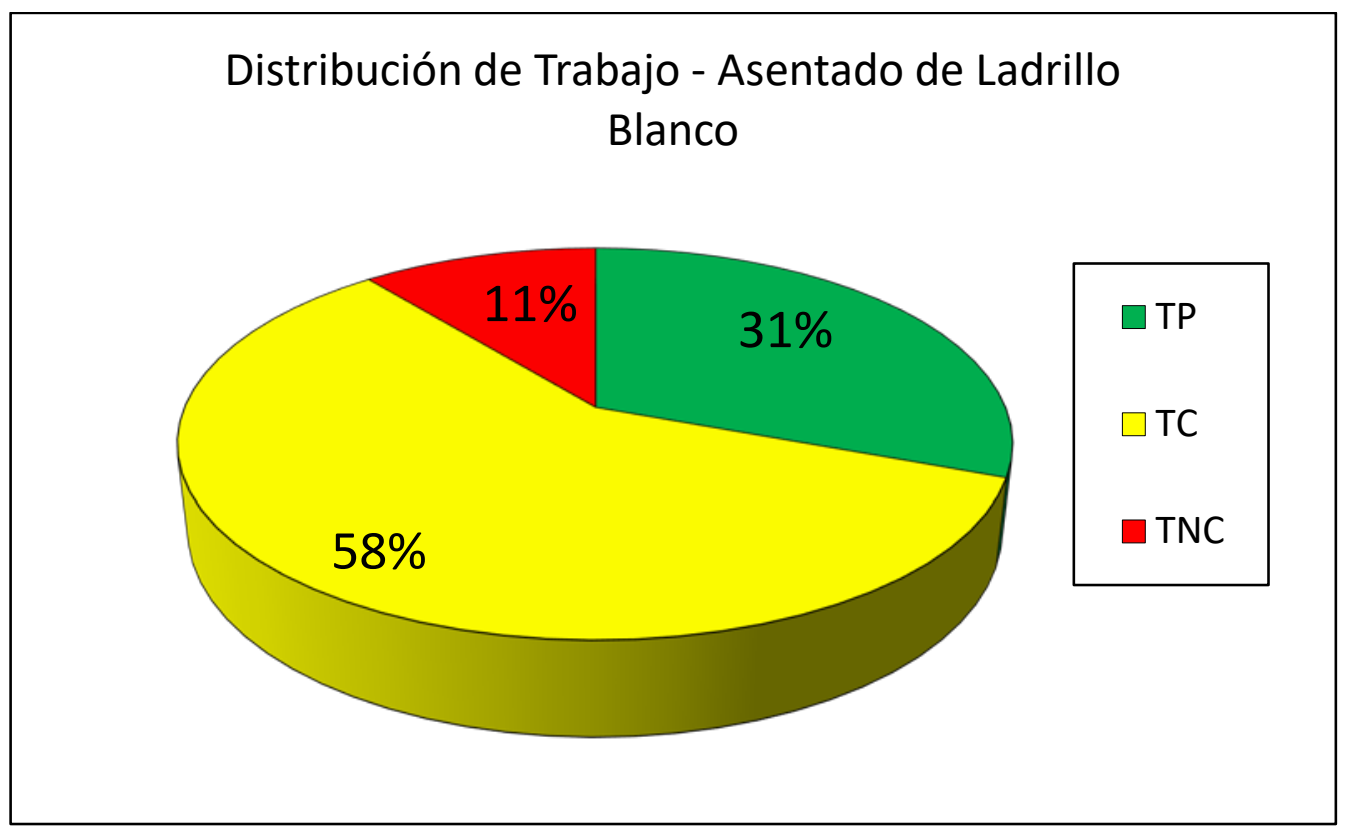

Fuente: Elaboración Propia.

Figura Nº 76: Distribución de Trabajo Productivo - Carta Balance ${ }^{\circ} 23$

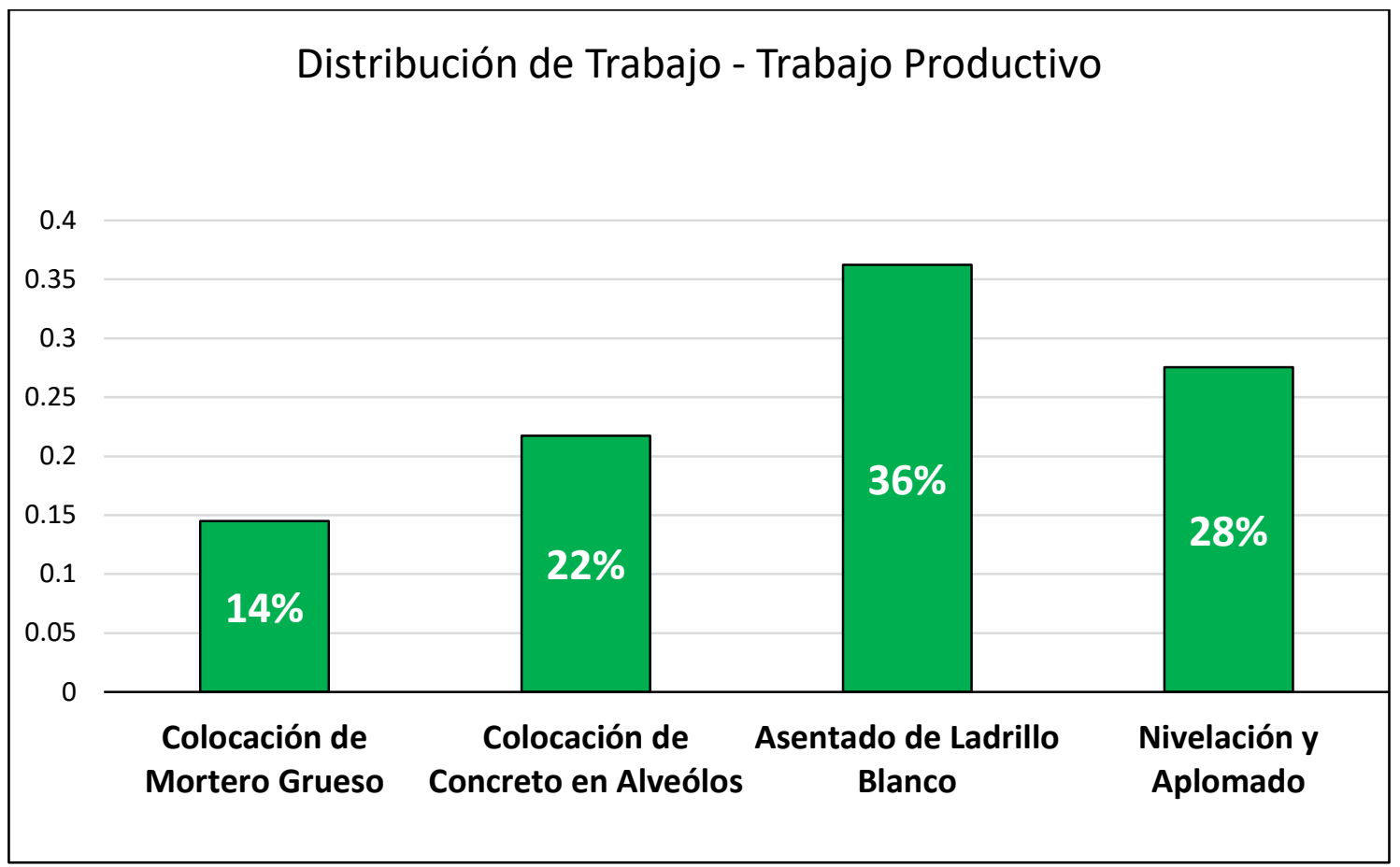

Fuente: Elaboración Propia.

Figura Nº 77: Distribución de Trabajo Contributorio - Carta Balance ${ }^{\circ} 23$ 


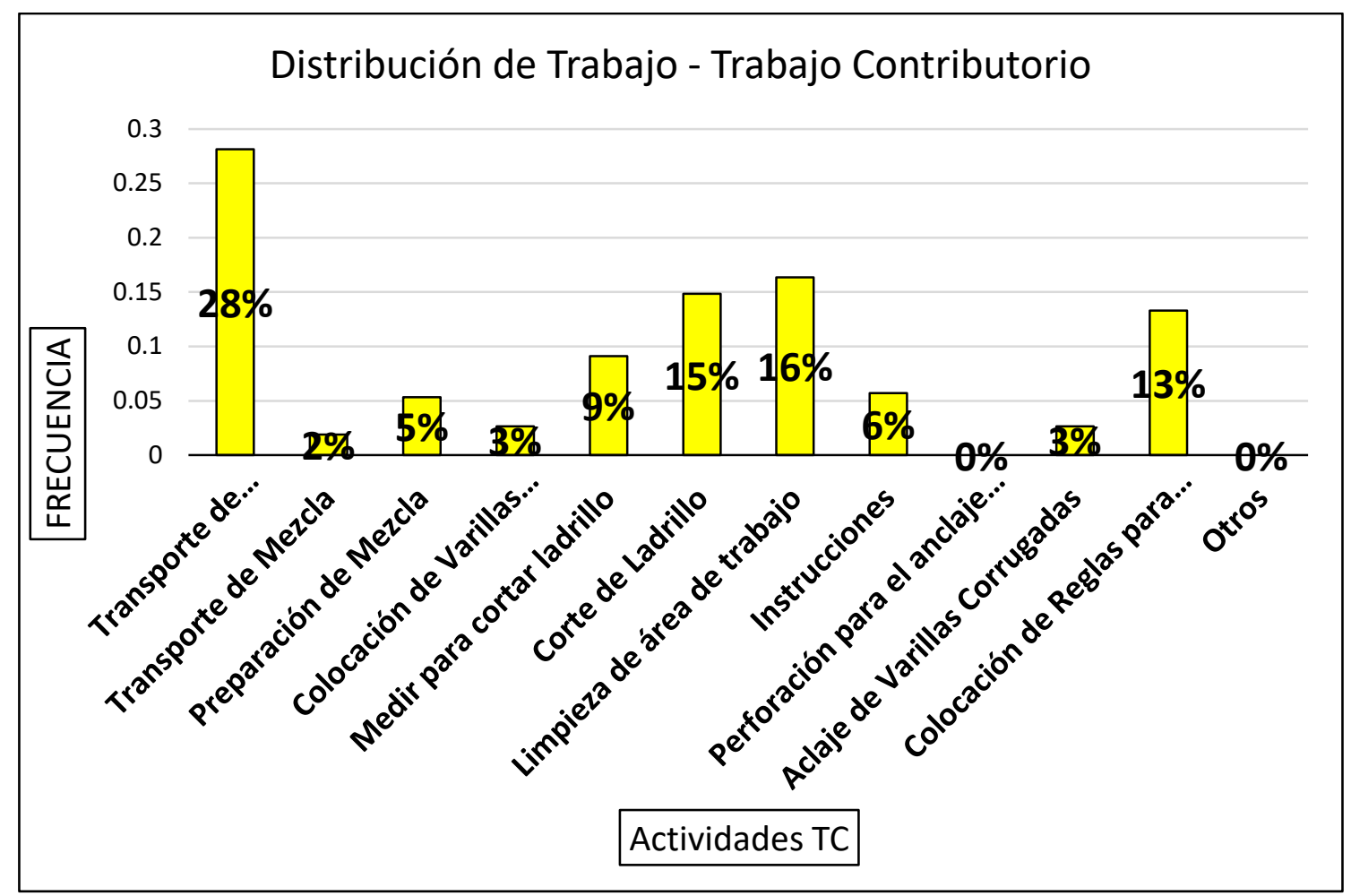

Fuente: Elaboración Propia.

Figura No 78: Distribución de Trabajo No Contributorio - Carta Balance ${ }^{\circ} 23$

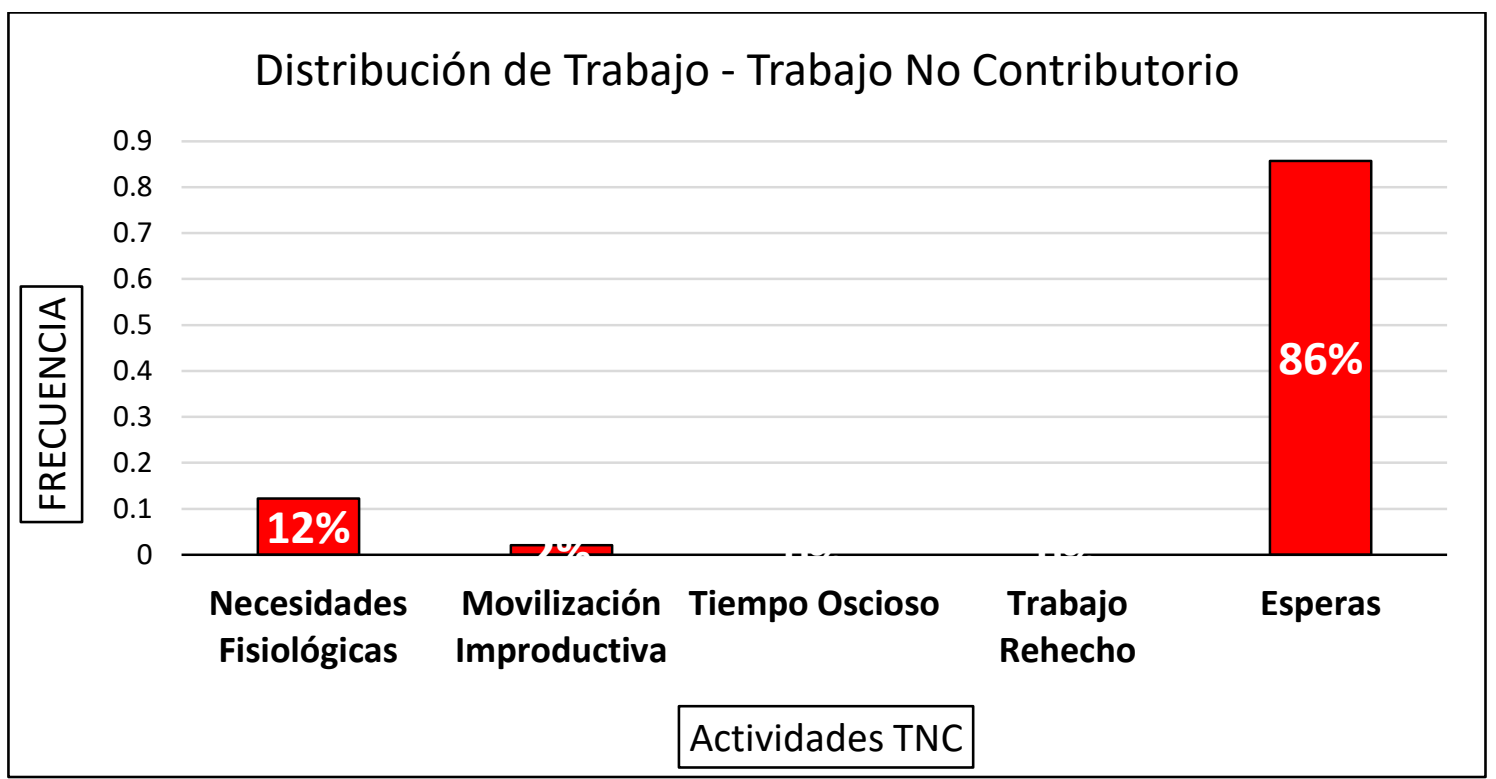

Fuente: Elaboración Propia. 
Tabla No 23: Carta Balance ${ }^{\circ} 24$ - Asentado de Ladrillo

\begin{tabular}{|c|c|c|c|c|c|c|c|c|c|c|c|}
\hline $\mathrm{N}^{0}$ & Obrero\#1 & Obrero\#2 & Obrero \#3 & Obrero\#4 & Obrero \#5 & 46 & $C R$ & NA & $E$ & $\mathrm{CL}$ & $\mathrm{TH}$ \\
\hline 1 & $C A$ & NA & NA & $\mathrm{CL}$ & $\mathrm{TH}$ & 47 & $C R$ & $\mathrm{AL}$ & $\mathrm{AL}$ & $\mathrm{CL}$ & $\mathrm{TH}$ \\
\hline 2 & $C A$ & NA & $C A$ & $\mathrm{CL}$ & $\mathrm{TH}$ & 48 & $C R$ & $\mathrm{AL}$ & NA & $\mathrm{CL}$ & $\mathrm{TH}$ \\
\hline 3 & $E$ & NA & $C A$ & $\mathrm{CL}$ & $\mathrm{TH}$ & 49 & $C R$ & $\mathrm{AL}$ & NA & ML & TO \\
\hline 4 & $\mathrm{CM}$ & NA & $C A$ & $\mathrm{CL}$ & $\mathrm{TH}$ & 50 & $C R$ & $\mathrm{AL}$ & NA & $\mathrm{ML}$ & TO \\
\hline 5 & $\mathrm{CM}$ & NA & PM & $\mathrm{CL}$ & $\mathrm{TH}$ & 51 & $C R$ & $\mathrm{AL}$ & NA & $\mathrm{CL}$ & TO \\
\hline 6 & $\mathrm{AL}$ & NA & AV & $\mathrm{CL}$ & $\mathrm{TH}$ & 52 & $C R$ & $\mathrm{AL}$ & $C A$ & $\mathrm{TH}$ & TO \\
\hline 7 & NA & NA & $\mathrm{CH}$ & $\mathrm{CL}$ & $\mathrm{L}$ & 53 & $C R$ & $\mathrm{AL}$ & $C A$ & $\mathrm{CL}$ & TO \\
\hline 8 & $C A$ & NA & PM & $\mathrm{CL}$ & $L$ & 54 & I & $\mathrm{AL}$ & $C A$ & $\mathrm{CL}$ & TO \\
\hline 9 & $C A$ & NA & $\mathrm{CM}$ & $\mathrm{CL}$ & $L$ & 55 & I & $\mathrm{AL}$ & $C A$ & TO & TO \\
\hline 10 & $\mathrm{CA}$ & NA & $\mathrm{CM}$ & $\mathrm{CL}$ & $L$ & 56 & $C R$ & NA & NA & TO & $\mathrm{TH}$ \\
\hline 11 & NA & NA & $\mathrm{CM}$ & $\mathrm{TH}$ & $\mathrm{L}$ & 57 & $C R$ & TO & $C A$ & $\mathrm{TH}$ & TO \\
\hline 12 & $\mathrm{AL}$ & PM & $\mathrm{CM}$ & $\mathrm{TH}$ & $\mathrm{L}$ & 58 & $C R$ & TO & TO & $\mathrm{CL}$ & TO \\
\hline 13 & NA & $C A$ & $\mathrm{AL}$ & $\mathrm{ML}$ & $\mathrm{L}$ & 59 & $C R$ & TO & TO & $\mathrm{Cl}$ & TO \\
\hline 14 & $C A$ & $C A$ & NA & $\mathrm{ML}$ & $\mathrm{L}$ & 60 & $C R$ & TO & TO & ML & TO \\
\hline 15 & $C A$ & $C A$ & $\mathrm{AL}$ & $\mathrm{ML}$ & $\mathrm{L}$ & 61 & $C R$ & $\mathrm{PM}$ & $\mathrm{AL}$ & $\mathrm{CL}$ & $\mathrm{L}$ \\
\hline 16 & $C A$ & $\mathrm{CH}$ & NA & $\mathrm{ML}$ & $\mathrm{L}$ & 62 & $C R$ & PM & NA & $\mathrm{ML}$ & $\mathrm{L}$ \\
\hline 17 & $\mathrm{~L}$ & $\mathrm{CH}$ & $\mathrm{AL}$ & $\mathrm{ML}$ & L & 63 & $\mathrm{TH}$ & $C A$ & $\mathrm{AL}$ & $\mathrm{CL}$ & $\mathrm{L}$ \\
\hline 18 & $\mathrm{~L}$ & $\mathrm{CM}$ & $\mathrm{AL}$ & $\mathrm{ML}$ & $\mathrm{L}$ & 64 & $\mathrm{TH}$ & $C A$ & $\mathrm{AL}$ & $\mathrm{CL}$ & $\mathrm{L}$ \\
\hline 19 & $\mathrm{~L}$ & $\mathrm{CM}$ & $\mathrm{AL}$ & $\mathrm{ML}$ & $L$ & 65 & $\mathrm{TH}$ & $C A$ & NA & $L$ & $\mathrm{~L}$ \\
\hline 20 & $L$ & $\mathrm{AL}$ & $\mathrm{AL}$ & $\mathrm{ML}$ & $L$ & 66 & $\mathrm{TH}$ & $C A$ & $\mathrm{AL}$ & $\mathrm{Cl}$ & $\mathrm{L}$ \\
\hline 21 & L & $\mathrm{AL}$ & NA & $\mathrm{ML}$ & $\mathrm{L}$ & 67 & $L$ & $\mathrm{~L}$ & $\mathrm{AL}$ & $\mathrm{Cl}$ & $\mathrm{L}$ \\
\hline 22 & $L$ & $\mathrm{AL}$ & NA & $\mathrm{ML}$ & $L$ & 68 & $\mathrm{TH}$ & $\mathrm{CH}$ & $\mathrm{AL}$ & $\mathrm{CL}$ & $\mathrm{L}$ \\
\hline 23 & $L$ & $\mathrm{AL}$ & NA & $\mathrm{CL}$ & $L$ & 69 & $L$ & $\mathrm{PM}$ & NA & $\mathrm{CL}$ & $\mathrm{L}$ \\
\hline 24 & L & $\mathrm{AL}$ & NA & $\mathrm{TH}$ & $\mathrm{TH}$ & 70 & $L$ & $\mathrm{PM}$ & $\mathrm{CA}$ & $L$ & $\mathrm{~L}$ \\
\hline 25 & $\mathrm{~L}$ & $\mathrm{AL}$ & $\mathrm{PM}$ & $\mathrm{TH}$ & TH & 71 & $L$ & $\mathrm{CM}$ & $C A$ & $\mathrm{CL}$ & TH \\
\hline 26 & $C R$ & $\mathrm{AL}$ & $C A$ & $\mathrm{CL}$ & $\mathrm{CL}$ & 72 & $C R$ & $\mathrm{CM}$ & $C A$ & $\mathrm{Cl}$ & TH \\
\hline 27 & $C R$ & $\mathrm{AL}$ & $C A$ & $\mathrm{TH}$ & $\mathrm{CL}$ & 73 & $C R$ & $\mathrm{AL}$ & $C A$ & $\mathrm{ML}$ & $\mathrm{TH}$ \\
\hline 28 & $C R$ & $\mathrm{AL}$ & $\mathrm{CA}$ & $\mathrm{CL}$ & $\mathrm{CL}$ & 74 & $C R$ & $\mathrm{AL}$ & $C A$ & $\mathrm{ML}$ & $\mathrm{TH}$ \\
\hline 29 & $C R$ & NA & $C A$ & $\mathrm{CL}$ & $\mathrm{CL}$ & 75 & $C R$ & $\mathrm{AL}$ & $\mathrm{CA}$ & $\mathrm{CL}$ & $\mathrm{TH}$ \\
\hline 30 & $C R$ & NA & 1 & $\mathrm{CL}$ & $\mathrm{TH}$ & 76 & $C R$ & $\mathrm{AL}$ & $C A$ & $\mathrm{CL}$ & $\mathrm{TH}$ \\
\hline 31 & $L$ & NA & 1 & $\mathrm{CL}$ & $\mathrm{TH}$ & 77 & $\mathrm{CM}$ & $\mathrm{AL}$ & $\mathrm{L}$ & $\mathrm{ML}$ & $\mathrm{TH}$ \\
\hline 32 & $\mathrm{~L}$ & NA & $\mathrm{CA}$ & $\mathrm{ML}$ & $\mathrm{TH}$ & 78 & $\mathrm{CM}$ & $\mathrm{AL}$ & NA & $\mathrm{ML}$ & $\mathrm{TH}$ \\
\hline 33 & $L$ & $\mathrm{PM}$ & $\mathrm{CA}$ & $\mathrm{CL}$ & $\mathrm{TH}$ & 79 & $\mathrm{CM}$ & $\mathrm{AL}$ & NA & $\mathrm{ML}$ & $\mathrm{TH}$ \\
\hline 34 & $\mathrm{TH}$ & $C A$ & $C A$ & $\mathrm{CL}$ & $\mathrm{TH}$ & 80 & $\mathrm{CM}$ & $\mathrm{AL}$ & NA & $\mathrm{ML}$ & $\mathrm{TH}$ \\
\hline 35 & $\mathrm{TH}$ & $C A$ & $\mathrm{CM}$ & $E$ & $\mathrm{TH}$ & 81 & NA & $\mathrm{AL}$ & NA & $\mathrm{Cl}$ & $\mathrm{TH}$ \\
\hline 36 & $\mathrm{~L}$ & $C A$ & NA & $E$ & TH & 82 & NA & $\mathrm{AL}$ & NA & $\mathrm{Cl}$ & $\mathrm{TH}$ \\
\hline 37 & $\mathrm{~L}$ & $C A$ & $\mathrm{AL}$ & $\mathrm{CL}$ & $\mathrm{TH}$ & 83 & $\mathrm{AL}$ & NA & $\mathrm{L}$ & $\mathrm{ML}$ & $\mathrm{TH}$ \\
\hline 38 & 1 & $\mathrm{PM}$ & $\mathrm{AL}$ & $\mathrm{CL}$ & $\mathrm{TH}$ & 84 & $\mathrm{AL}$ & NA & $L$ & $\mathrm{ML}$ & $\mathrm{TH}$ \\
\hline 39 & $C R$ & $\mathrm{PM}$ & $\mathrm{AL}$ & $\mathrm{CL}$ & $\mathrm{TH}$ & 85 & $\mathrm{AL}$ & NA & $\mathrm{L}$ & $\mathrm{ML}$ & $\mathrm{TH}$ \\
\hline 40 & $C R$ & PM & $\mathrm{AL}$ & $\mathrm{CL}$ & $\mathrm{TH}$ & 86 & $\mathrm{TH}$ & $\mathrm{PM}$ & $\mathrm{L}$ & $\mathrm{ML}$ & $\mathrm{TH}$ \\
\hline 41 & $C R$ & $\mathrm{~L}$ & $\mathrm{AL}$ & $\mathrm{ML}$ & $\mathrm{TH}$ & 87 & $\mathrm{TH}$ & $C A$ & $\mathrm{~L}$ & $\mathrm{CL}$ & $\mathrm{TH}$ \\
\hline 42 & $C R$ & PM & $\mathrm{AL}$ & $\mathrm{TH}$ & $\mathrm{TH}$ & 88 & NA & $C A$ & L & $\mathrm{ML}$ & $\mathrm{TH}$ \\
\hline 43 & $C R$ & $\mathrm{CM}$ & NA & $\mathrm{CL}$ & $\mathrm{L}$ & 89 & NA & PM & $\mathrm{L}$ & $\mathrm{CL}$ & $\mathrm{TH}$ \\
\hline 44 & $C R$ & $\mathrm{CM}$ & $\mathrm{AL}$ & $\mathrm{CL}$ & $L$ & 90 & $\mathrm{PM}$ & AV & $\mathrm{L}$ & $\mathrm{ML}$ & $\mathrm{TH}$ \\
\hline 45 & $C R$ & $\mathrm{AL}$ & $\mathrm{AL}$ & $\mathrm{CL}$ & $\mathrm{TH}$ & & & & & & \\
\hline
\end{tabular}

Fuente: Elaboración Propia. 
Figura N 79: Distribución de Trabajo de Asentado de Ladrillo - Carta Balance ${ }^{\circ} 24$

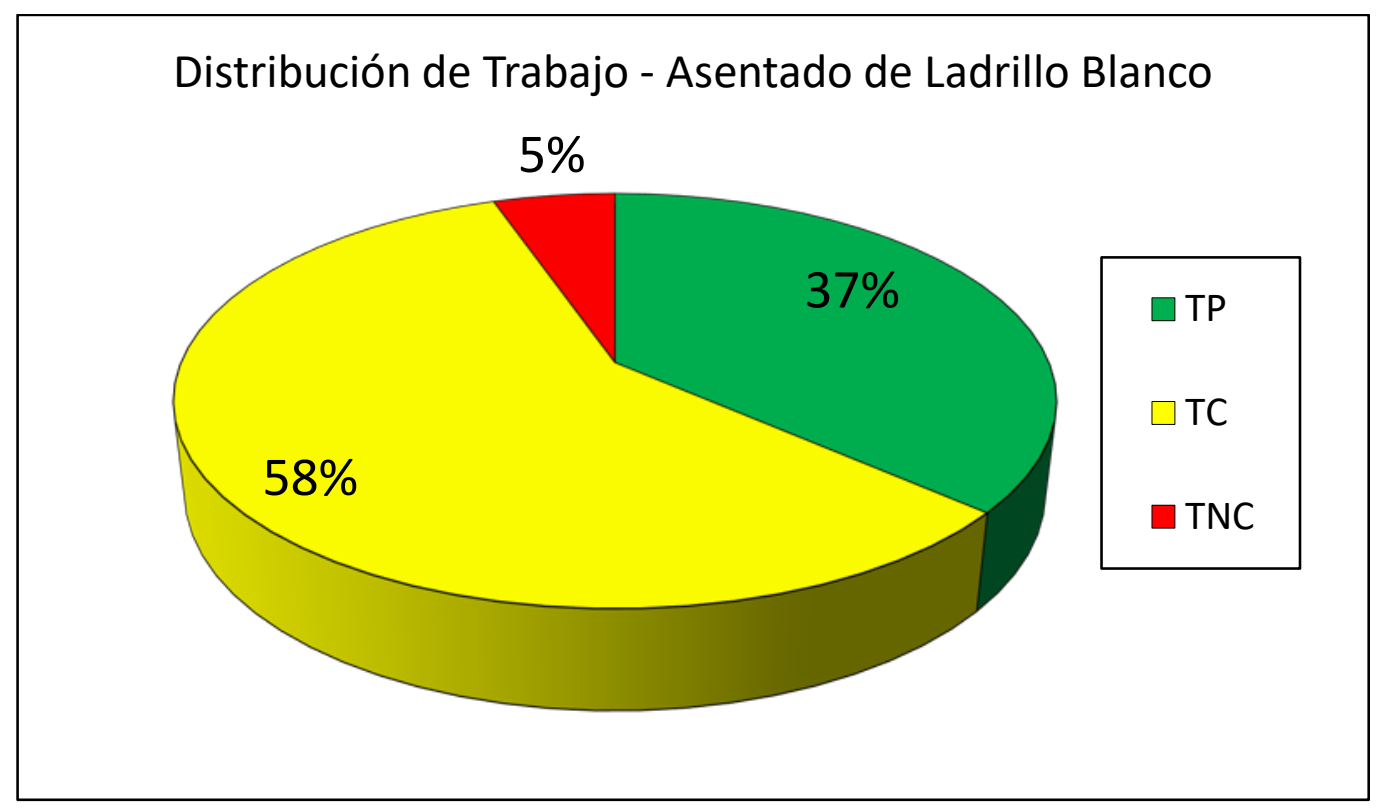

Fuente: Elaboración Propia.

Figura N 80: Distribución de Trabajo Productivo - Carta Balance ${ }^{\circ} 24$

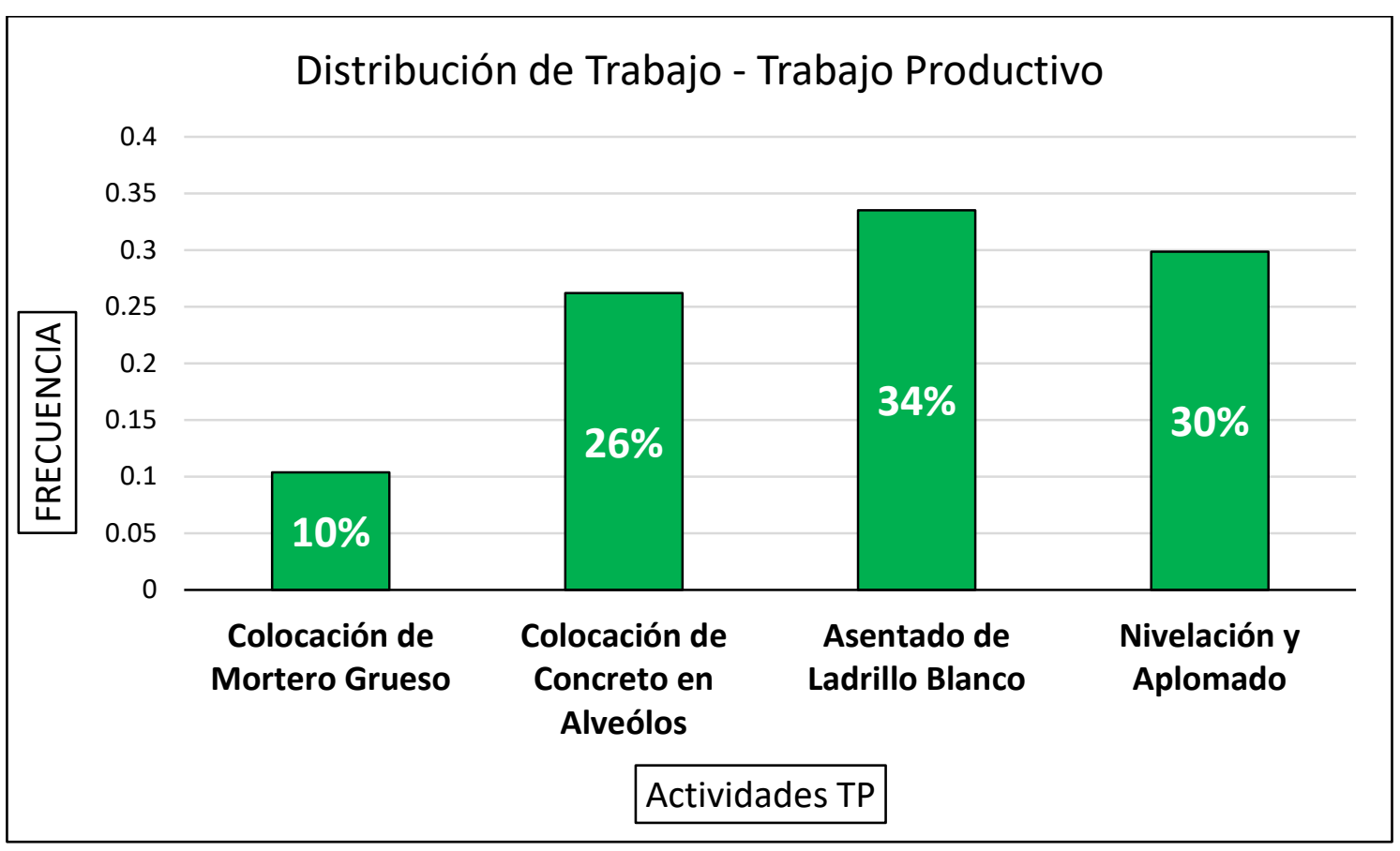

Fuente: Elaboración Propia. 
Figura Nº 81: Distribución de Trabajo Contributorio - Carta Balance ${ }^{\circ} 24$

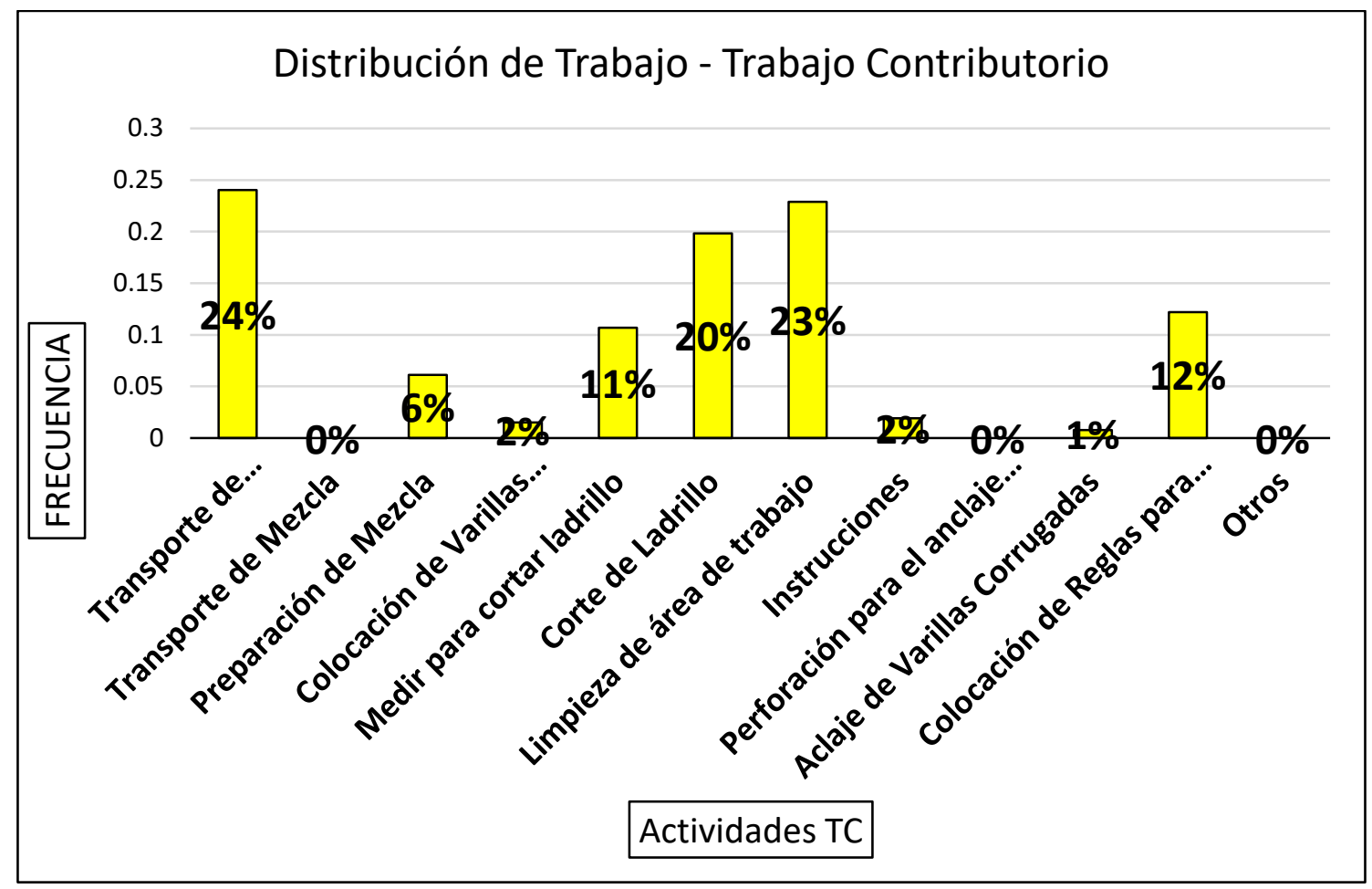

Fuente: Elaboración Propia.

Figura No 82: Distribución de Trabajo No Contributorio - Carta Balance ${ }^{\circ} 24$

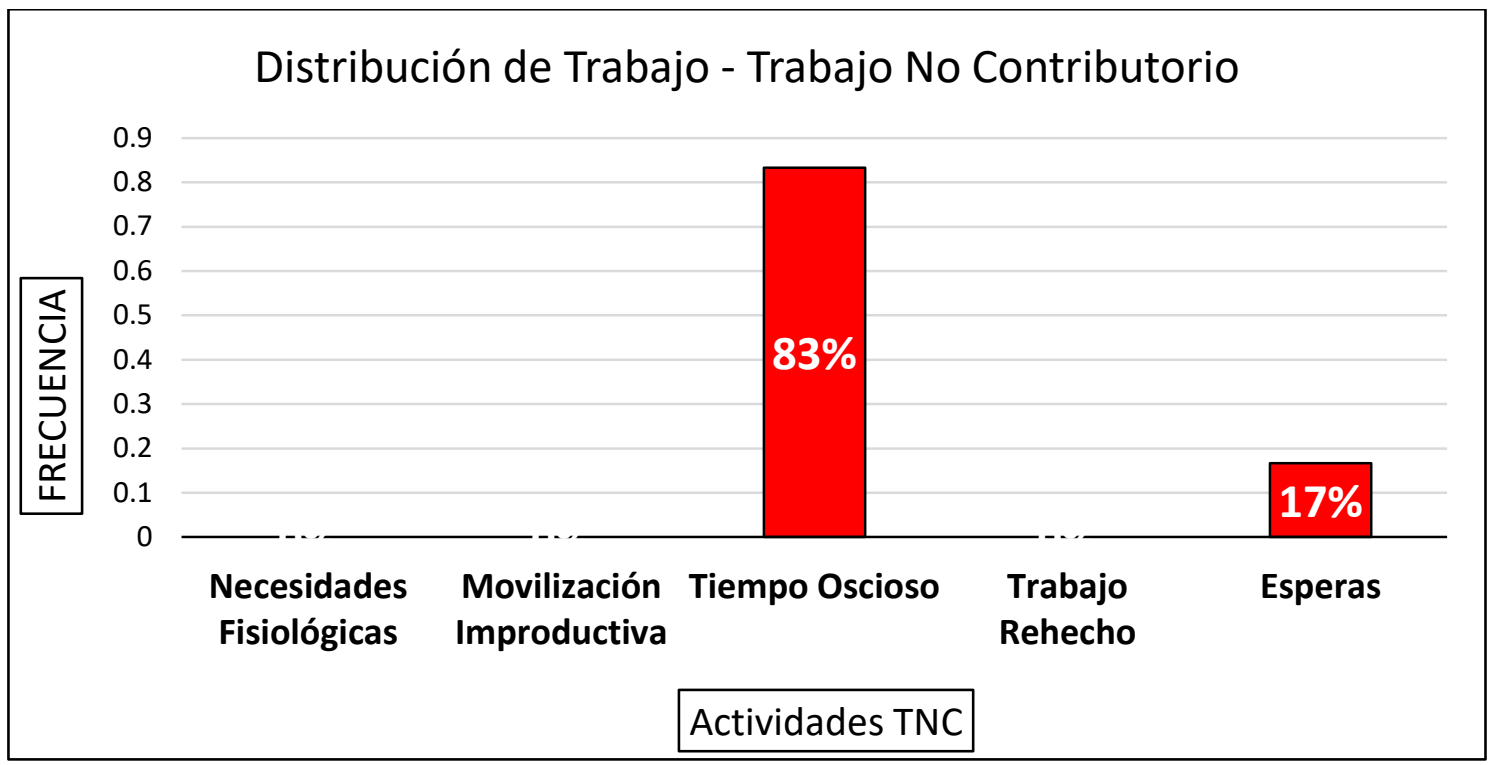

Fuente: Elaboración Propia. 
Tabla N 24: Carta Balance ${ }^{\circ} 9$ - Enchape de Cerámico

\begin{tabular}{|c|c|c|c|c|c|c|c|c|}
\hline $\mathrm{N}^{*}$ & Obrero \#1 & Obrero \#2 & 31 & MI & MI & 61 & $\mathrm{TM}$ & TM \\
\hline 1 & 1 & E & 32 & 1 & 1 & 62 & $\mathrm{TM}$ & TM \\
\hline 2 & $\mathrm{TM}$ & $E$ & 33 & MI & MI & 63 & $\mathrm{TM}$ & TM \\
\hline 3 & $\mathrm{TM}$ & E & 34 & MI & MI & 64 & $\mathrm{TM}$ & TM \\
\hline 4 & BM & $E$ & 35 & MI & MI & 65 & TM & TM \\
\hline 5 & COE & E & 36 & $\operatorname{COE}$ & E & 66 & TM & TO \\
\hline 6 & COE & $E$ & 37 & $\operatorname{coE}$ & $E$ & 67 & $\mathrm{TM}$ & TO \\
\hline 7 & COE & E & 38 & $C E$ & E & 68 & TM & TM \\
\hline 8 & TE & $E$ & 39 & $C P$ & TM & 69 & MI & TM \\
\hline 9 & $C P$ & E & 40 & $\operatorname{COE}$ & TM & 70 & $\mathrm{TM}$ & TM \\
\hline 10 & $C P$ & E & 41 & E & E & 71 & L & TO \\
\hline 11 & $C P$ & $E$ & 42 & TM & $E$ & 72 & $\underline{t}$ & TO \\
\hline 12 & 1 & 1 & 43 & $\mathrm{TM}$ & $\mathbf{E}$ & 73 & 1 & TO \\
\hline 13 & 1 & 1 & 44 & MI & $E$ & 74 & $\mathrm{TM}$ & TO \\
\hline 14 & 1 & 1 & 45 & MI & MI & 75 & TE & TO \\
\hline 15 & $C P$ & MI & 46 & MI & $E$ & 76 & 1 & TO \\
\hline 16 & $\mathrm{CE}$ & E & 47 & MI & $E$ & 77 & $E$ & E \\
\hline 17 & $C P$ & E & 48 & MI & $E$ & 78 & 1 & $E$ \\
\hline 18 & $C P$ & E & 49 & MI & E & 79 & 1 & $\mathrm{E}$ \\
\hline 19 & AP & E & 50 & MI & MI & 80 & 1 & $E$ \\
\hline 20 & AP & $\mathrm{E}$ & 51 & MI & MI & 81 & $\mathrm{E}$ & $\mathrm{E}$ \\
\hline 21 & $C P$ & E & 52 & MI & MI & 82 & 1 & E \\
\hline 22 & $C P$ & $\mathrm{E}$ & 53 & MI & MI & 83 & 1 & $\mathrm{E}$ \\
\hline 23 & AP & E & 54 & MI & MI & 84 & 1 & L \\
\hline 24 & $C C$ & $E$ & SS & MI & MI & 85 & 1 & MI \\
\hline 25 & 1 & 1 & 56 & $\mathrm{TM}$ & MI & 86 & 1 & MI \\
\hline 26 & COE & $E$ & 57 & 1 & MI & 87 & 1 & MI \\
\hline 27 & $\mathrm{COE}$ & $\mathrm{E}$ & 58 & 1 & $E$ & 88 & 1 & MI \\
\hline 28 & MI & MI & 59 & 1 & $E$ & 89 & 1 & MI \\
\hline 29 & MI & MI & 60 & 1 & 1 & 90 & 1 & MI \\
\hline 30 & MI & MI & & & & & & \\
\hline
\end{tabular}

Fuente: Elaboración Propia. 
Figura Nº 83: Distribución de Trabajo de Enchape de Cerámico - Carta Balance ${ }^{\circ} 9$

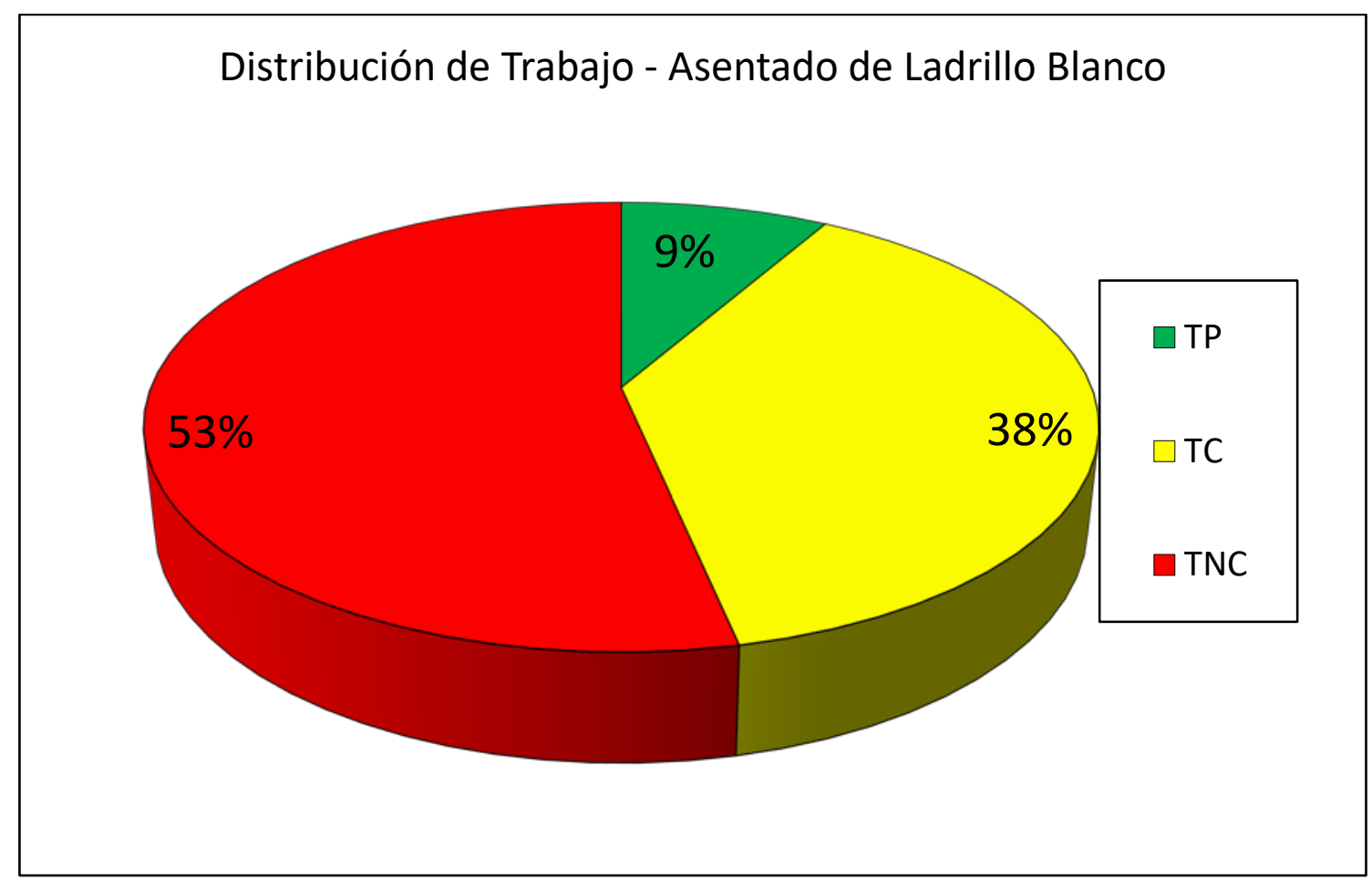

Fuente: Elaboración Propia.

Figura $N^{\circ}$ 84: Distribución de Trabajo Productivo - Carta Balance ${ }^{\circ 9}$

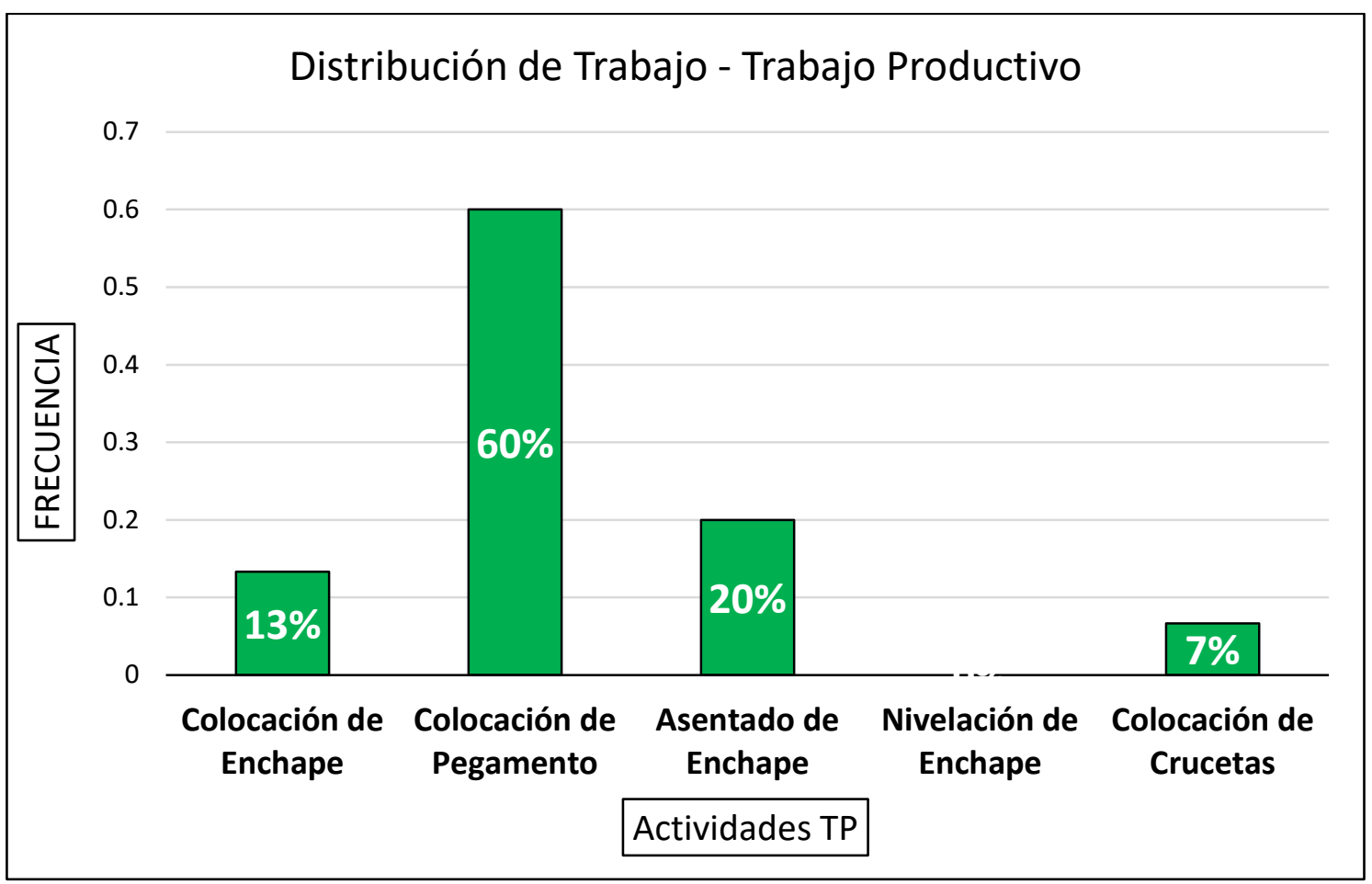

Fuente: Elaboración Propia. 
Figura Nº 85: Distribución de Trabajo Contributorio - Carta Balance ${ }^{\circ} 9$

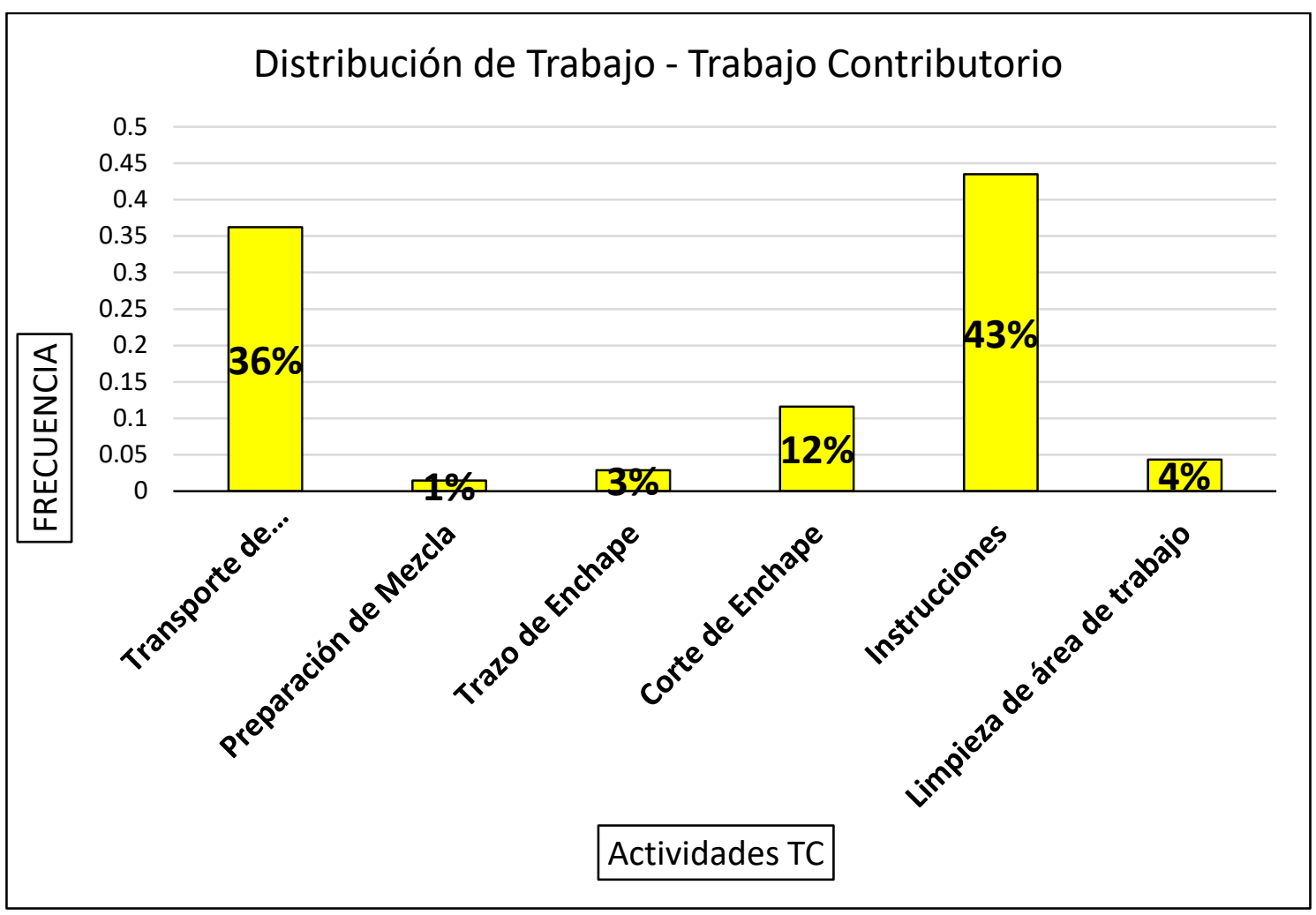

Fuente: Elaboración Propia.

Figura N 86: Distribución de Trabajo No Contributorio - Carta Balance ${ }^{\circ} 9$

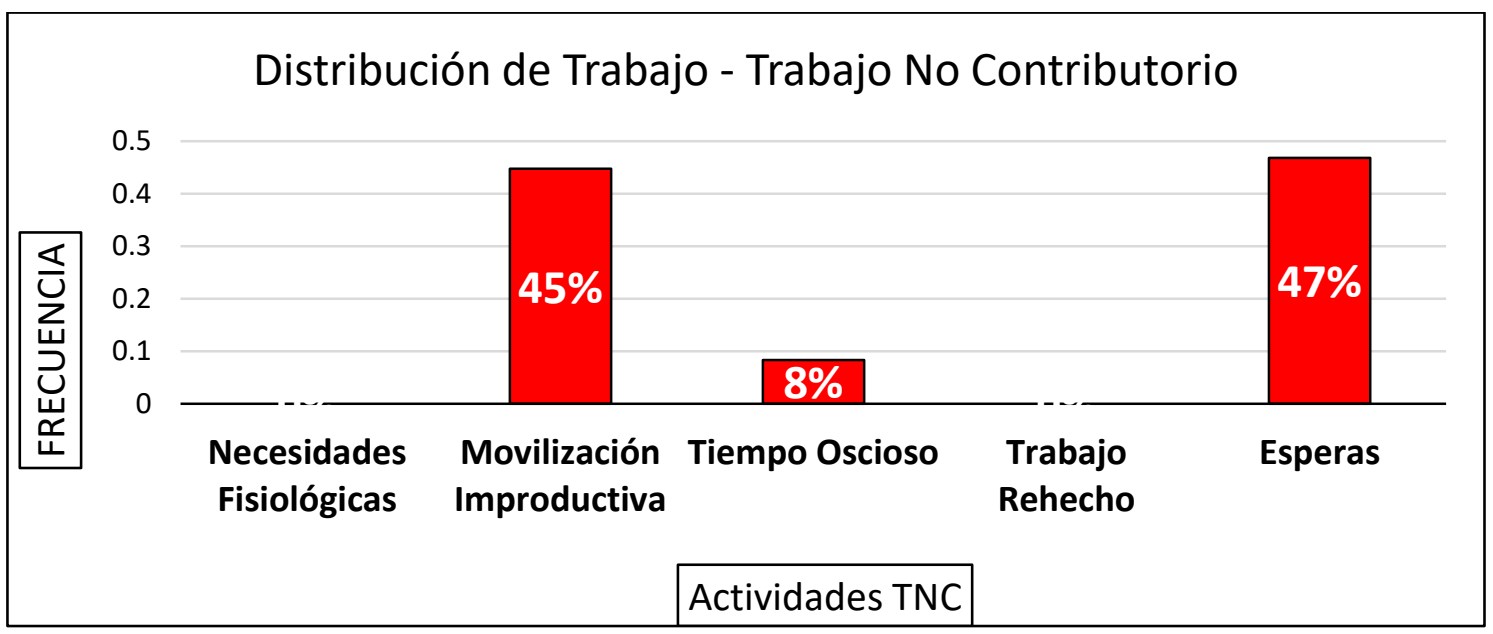

Fuente: Elaboración Propia. 
Tabla Nº 25: Carta Balance 10 - Enchape de Cerámico

\begin{tabular}{|c|c|c|c|c|c|c|c|c|}
\hline $\mathrm{N}^{*}$ & Obrero $\| 1$ & Obrero & 31 & $A P$ & $\mathrm{~L}$ & 61 & $C P$ & $E$ \\
\hline 1 & BM & $E$ & 32 & TE & L & 62 & $\mathrm{TE}$ & $E$ \\
\hline 2 & $\mathrm{TM}$ & L & 33 & CE & $\mathrm{TM}$ & 63 & CE & $E$ \\
\hline 3 & TE & $\mathrm{TM}$ & 34 & $A P$ & $\mathrm{TM}$ & 64 & TE & $E$ \\
\hline 4 & TE & $\mathrm{TM}$ & 35 & $\mathrm{CC}$ & $\mathrm{TM}$ & 65 & $\mathrm{CC}$ & $E$ \\
\hline 5 & COE & L & 36 & $\mathrm{CC}$ & $\mathrm{TM}$ & 66 & NE & $E$ \\
\hline 6 & COE & $\mathrm{L}$ & 37 & $C C$ & $\mathrm{TM}$ & 67 & TE & $E$ \\
\hline 7 & COE & L & 38 & $C C$ & $\mathrm{TM}$ & 68 & TE & $E$ \\
\hline 8 & COE & I & 39 & TE & $\mathrm{TM}$ & 69 & COE & E \\
\hline 9 & $C P$ & $\mathrm{l}$ & 40 & TE & $\mathrm{TM}$ & 70 & COE & $\mathrm{E}$ \\
\hline 10 & $C P$ & L & 41 & $\operatorname{COE}$ & $\mathrm{TM}$ & 71 & $\operatorname{COE}$ & $E$ \\
\hline 11 & CE & L & 42 & $\mathrm{CC}$ & $E$ & 72 & 1 & $E$ \\
\hline 12 & CE & L & 43 & TE & $E$ & 73 & $\mathrm{TM}$ & $E$ \\
\hline 13 & $A P$ & L & 44 & $\mathrm{CC}$ & $E$ & 74 & $\mathrm{TM}$ & $\mathrm{TM}$ \\
\hline 14 & $C E$ & L & 45 & $A P$ & $E$ & 75 & $\operatorname{COE}$ & $E$ \\
\hline 15 & AP & L & 46 & NE & $E$ & 76 & COE & $E$ \\
\hline 16 & CE & $\mathrm{TM}$ & 47 & NE & $E$ & 77 & COE & E \\
\hline 17 & COE & TM & 48 & NE & $E$ & 78 & TE & E \\
\hline 18 & $C E$ & E & 49 & NE & $E$ & 79 & $\mathrm{COE}$ & $E$ \\
\hline 19 & AP & E & 50 & TE & $E$ & 80 & COE & $E$ \\
\hline 20 & AP & $E$ & 51 & $A P$ & $E$ & 81 & $C P$ & $E$ \\
\hline 21 & AP & $E$ & 52 & $C C$ & $E$ & 82 & $\mathrm{CP}$ & $\mathrm{E}$ \\
\hline 22 & $C C$ & $\mathrm{E}$ & 53 & COE & $E$ & 83 & $\mathrm{CE}$ & $\mathrm{E}$ \\
\hline 23 & $\mathrm{CC}$ & $\mathrm{E}$ & 54 & COE & $E$ & 84 & $\mathrm{CE}$ & $E$ \\
\hline 24 & TE & $E$ & 5S & $\mathrm{CC}$ & $E$ & 85 & $A P$ & $E$ \\
\hline 25 & TE & $E$ & 56 & L & $E$ & 86 & TE & $E$ \\
\hline 26 & COE & E & 57 & TR & $E$ & 87 & $\mathrm{TE}$ & $E$ \\
\hline 27 & TE & L & 58 & 1 & 1 & 88 & COE & $E$ \\
\hline 28 & $C P$ & L & 59 & TE & $E$ & 89 & $A P$ & TM \\
\hline 29 & $C P$ & L & 60 & $C P$ & $E$ & 90 & NE & TM \\
\hline 30 & $C E$ & l & & & & & & \\
\hline
\end{tabular}

Fuente: Elaboración Propia. 
Figura Nº 87: Distribución de Trabajo de Enchape de Cerámico - Carta Balance `10

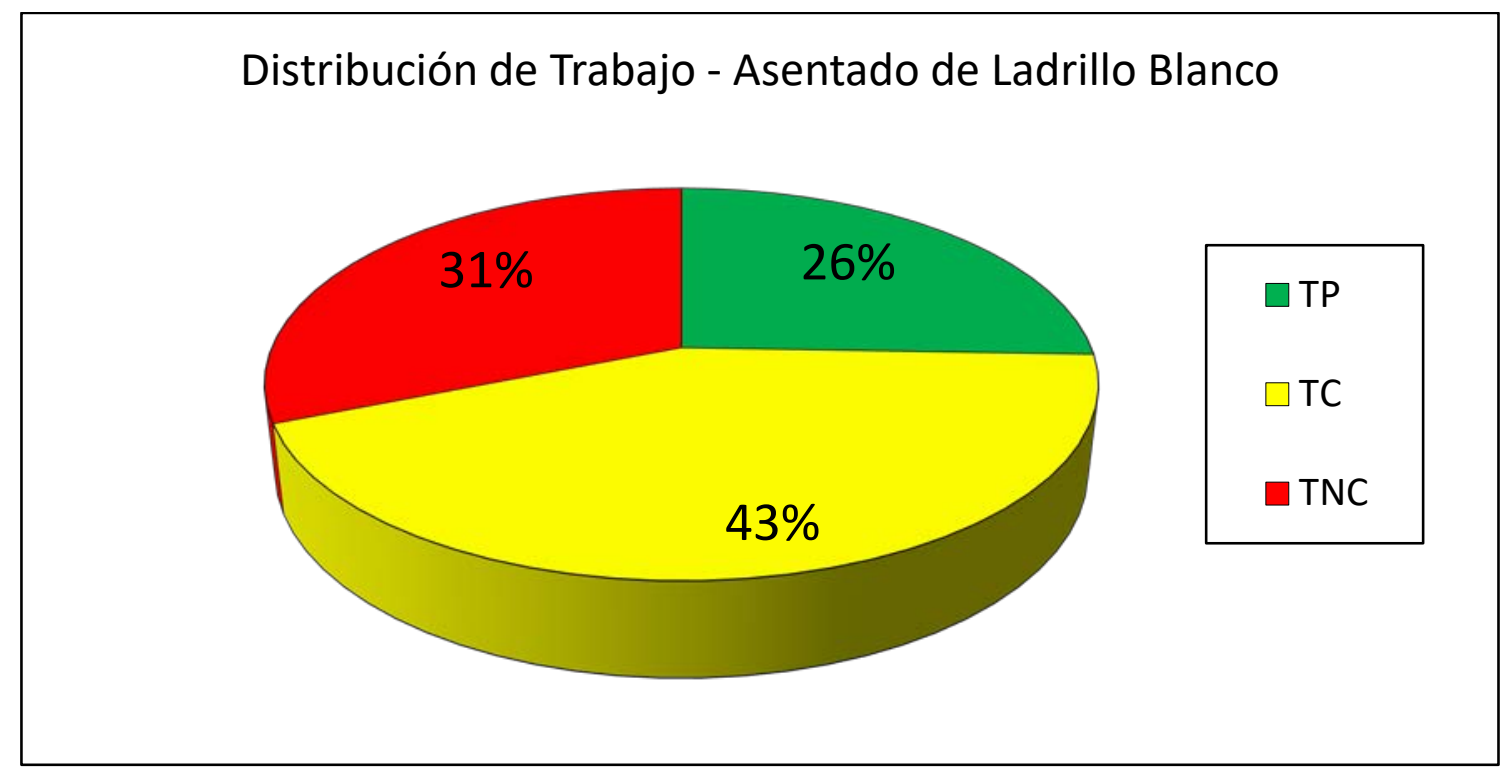

Fuente: Elaboración Propia.

Figura $N^{\circ}$ 88: Distribución de Trabajo Productivo - Carta Balance ${ }^{\circ} 10$

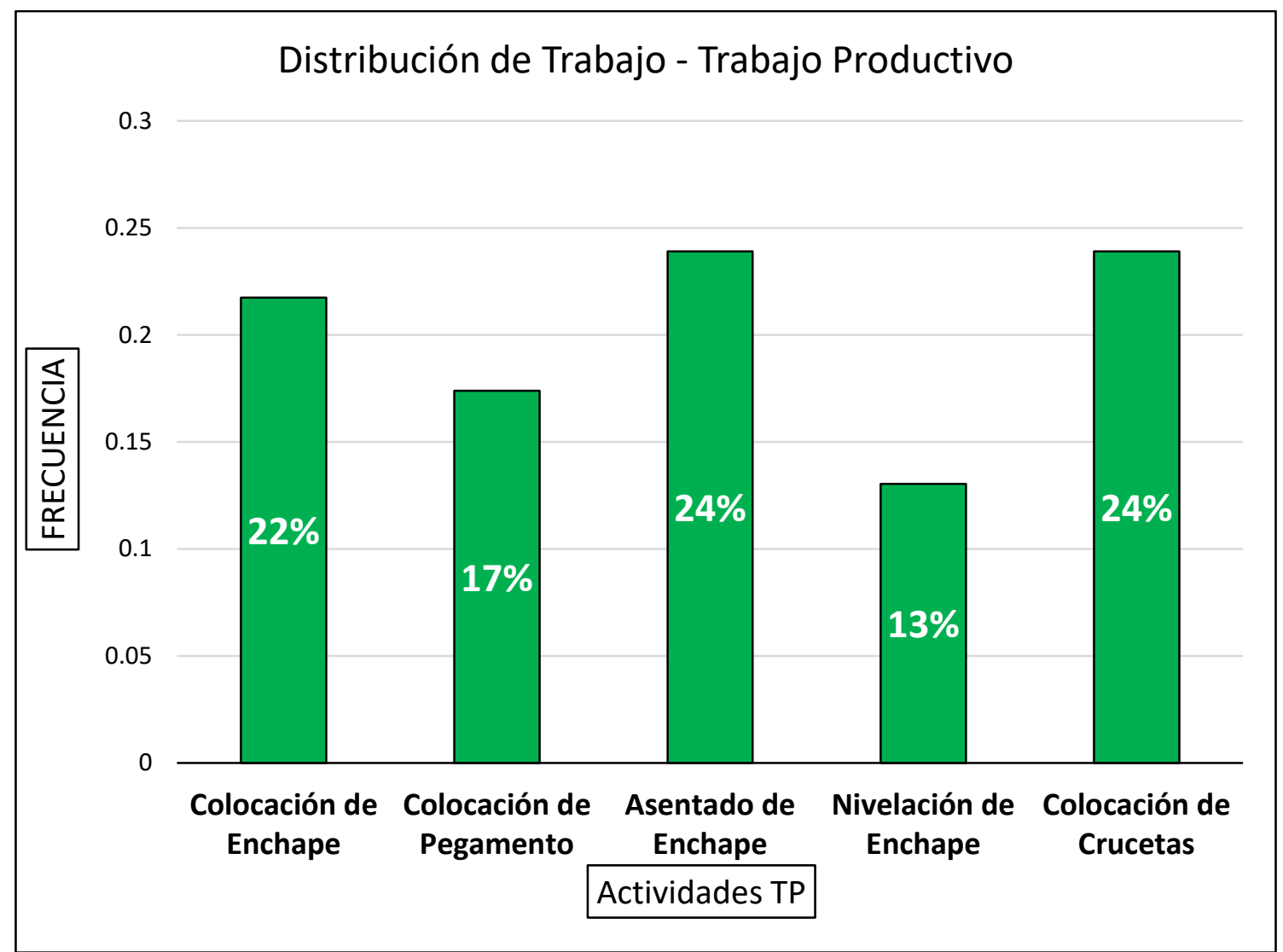

Fuente: Elaboración Propia. 
Figura Nº 89: Distribución de Trabajo Contributorio - Carta Balance ${ }^{\circ} 10$

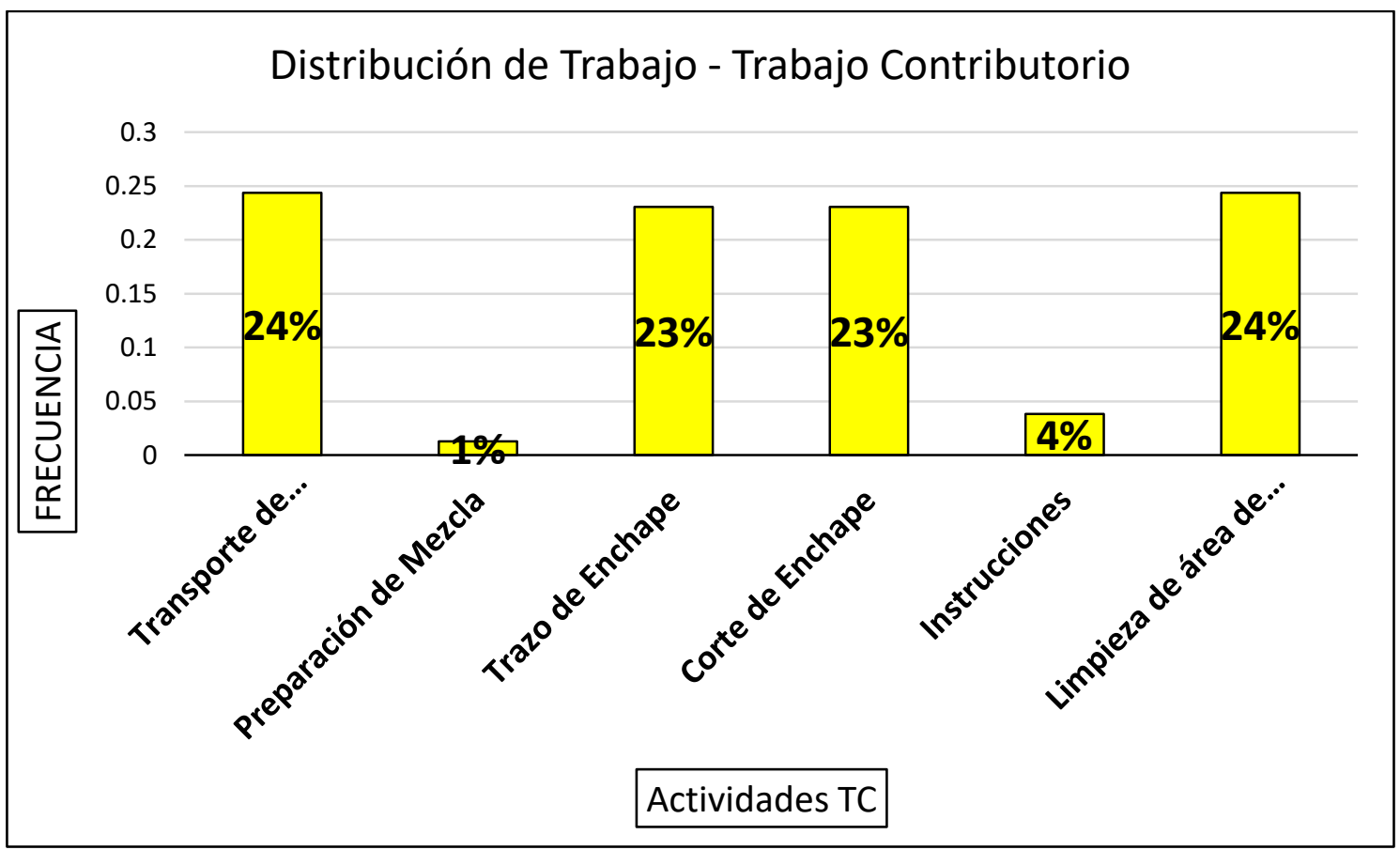

Fuente: Elaboración Propia.

Figura N 90: Distribución de Trabajo No Contributorio - Carta Balance ${ }^{\circ} 10$

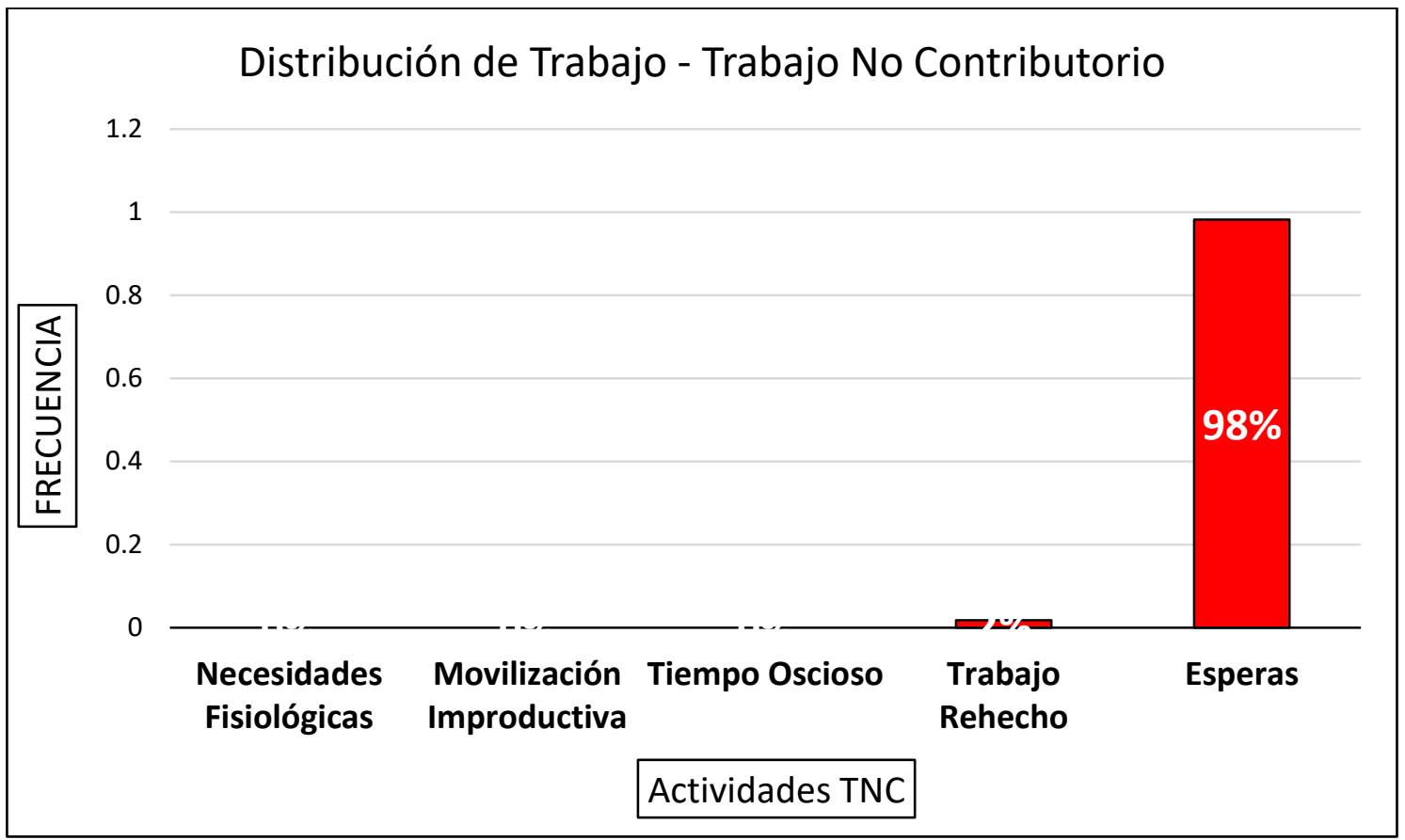

Fuente: Elaboración Propia. 
Tabla N² 26: Carta Balance ${ }^{\circ} 11$ - Enchape de Cerámico

\begin{tabular}{|c|c|c|c|c|c|c|c|c|}
\hline $\mathrm{N}^{*}$ & Obrero \#1 & Obrero $\# 2$ & 31 & $\mathrm{CE}$ & $E$ & 61 & $C P$ & $E$ \\
\hline 1 & AP & E & 32 & $C P$ & E & 62 & $C C$ & E \\
\hline 2 & $\mathrm{COE}$ & E & 33 & $L$ & $E$ & 63 & $C P$ & E \\
\hline 3 & NE & E & 34 & BM & $E$ & 64 & AP & $\mathrm{E}$ \\
\hline 4 & TE & E & 35 & $\mathrm{TM}$ & E & 65 & $C P$ & $\mathrm{E}$ \\
\hline 5 & $\mathrm{COE}$ & $\mathrm{E}$ & 36 & $\mathrm{TM}$ & $E$ & 66 & $\mathrm{CC}$ & $\mathrm{E}$ \\
\hline 6 & $\mathrm{COE}$ & $\mathrm{E}$ & 37 & $\mathrm{TM}$ & TM & 67 & $A P$ & $\mathrm{E}$ \\
\hline 7 & $C P$ & $\mathrm{E}$ & 38 & L & TM & 68 & NE & $\mathrm{E}$ \\
\hline 8 & NE & $E$ & 39 & L & $E$ & 69 & TE & $E$ \\
\hline 9 & NE & $E$ & 40 & $t$ & $E$ & 70 & $\operatorname{COE}$ & $E$ \\
\hline 10 & CE & $E$ & 41 & $L$ & $E$ & 71 & $\mathrm{COE}$ & $E$ \\
\hline 11 & $A P$ & E & 42 & TE & $E$ & 72 & TE & E \\
\hline 12 & TM & E & 43 & TE & E & 73 & $C P$ & $E$ \\
\hline 13 & $A P$ & E & 44 & COE & E & 74 & $C E$ & $E$ \\
\hline 14 & $A P$ & E & 45 & COE & $E$ & 75 & $C P$ & E \\
\hline 15 & AP & $E$ & 46 & $C P$ & E & 76 & NE & E \\
\hline 16 & TE & $E$ & 47 & $C P$ & $E$ & 77 & $C C$ & E \\
\hline 17 & $C P$ & $\mathrm{E}$ & 48 & $C C$ & $E$ & 78 & $\mathrm{TM}$ & $\mathrm{E}$ \\
\hline 18 & $\mathrm{CE}$ & $\mathrm{E}$ & 49 & AP & E & 79 & $C P$ & $\mathrm{E}$ \\
\hline 19 & AP & $\mathrm{E}$ & 50 & $\mathrm{CC}$ & $\mathrm{E}$ & 80 & $C P$ & $\mathrm{E}$ \\
\hline 20 & NE & $\mathrm{E}$ & 51 & NE & $E$ & 81 & $\mathrm{CE}$ & $\mathrm{E}$ \\
\hline 21 & L & $\mathrm{E}$ & $\$ 2$ & TE & $E$ & 82 & $A P$ & $\mathrm{E}$ \\
\hline 22 & NE & $E$ & 53 & $\operatorname{COE}$ & $E$ & 83 & $C C$ & $\mathrm{E}$ \\
\hline 23 & $A P$ & $E$ & 54 & $L$ & $E$ & 84 & $C P$ & $E$ \\
\hline 24 & L & E & 55 & $C E$ & $E$ & 85 & $A P$ & $\mathrm{E}$ \\
\hline 25 & $C P$ & E & 56 & $C P$ & $E$ & 86 & $C C$ & $E$ \\
\hline 26 & AP & E & 57 & AP & $\mathbf{E}$ & 87 & $\mathrm{~L}$ & E \\
\hline 27 & $C P$ & E & 58 & NE & E & 88 & L & $\mathbf{E}$ \\
\hline 28 & TE & E & 59 & AP & E & 89 & $\mathrm{~L}$ & $E$ \\
\hline 29 & AP & $E$ & 60 & $C P$ & $E$ & 90 & $\mathrm{~L}$ & E \\
\hline 30 & TE & E & & & & & & \\
\hline
\end{tabular}

Fuente: Elaboración Propia. 
Figura Nº 91: Distribución de Trabajo de Enchape de Cerámico - Carta Balance ${ }^{\circ} 11$

Distribución de Trabajo - Asentado de Ladrillo Blanco

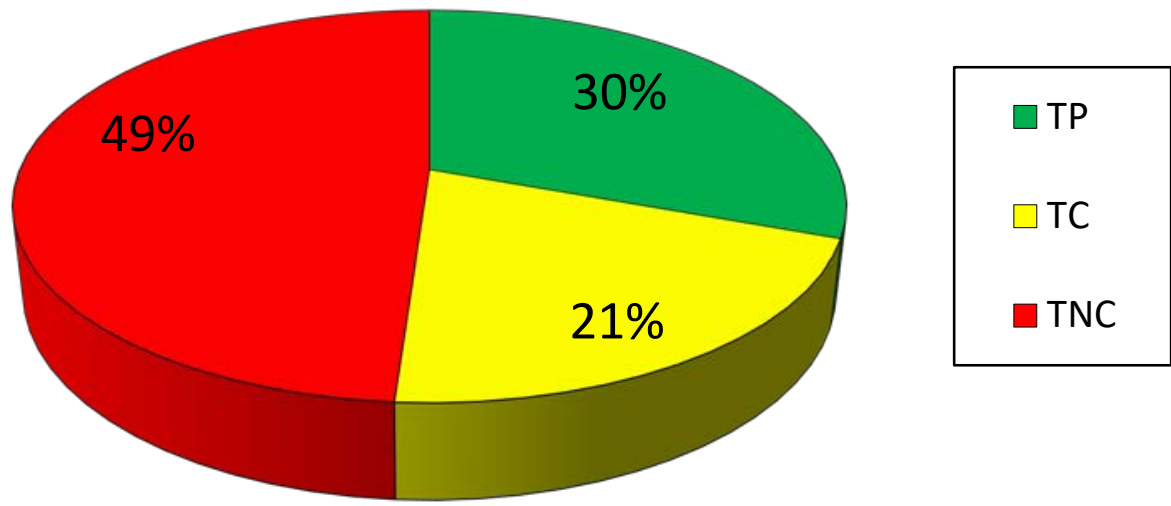

Fuente: Elaboración Propia.

Figura Nº 92: Distribución de Trabajo Productivo - Carta Balance ${ }^{\circ} 11$

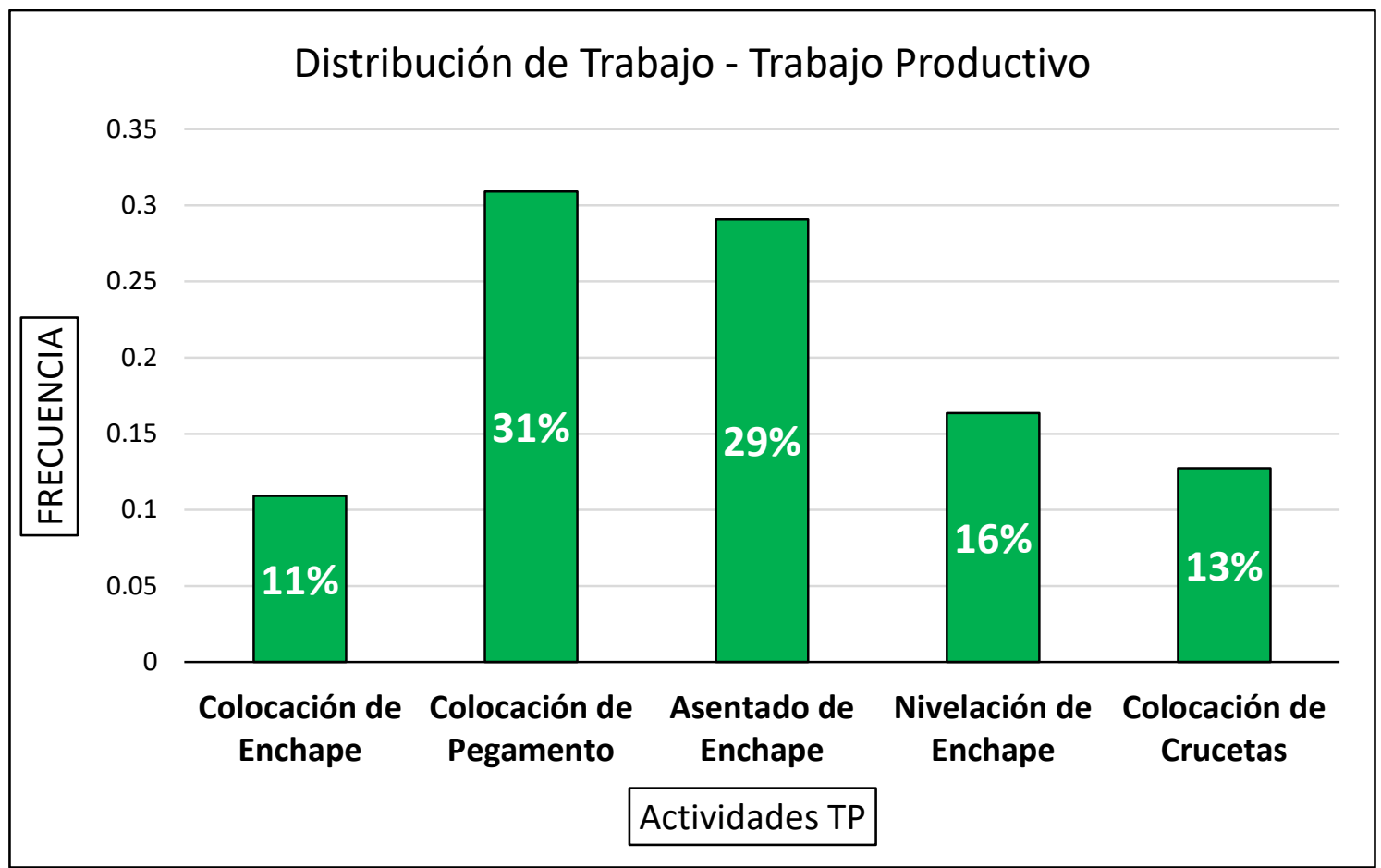

Fuente: Elaboración Propia. 
Figura N 93: Distribución de Trabajo Contributorio - Carta Balance ${ }^{\circ} 11$

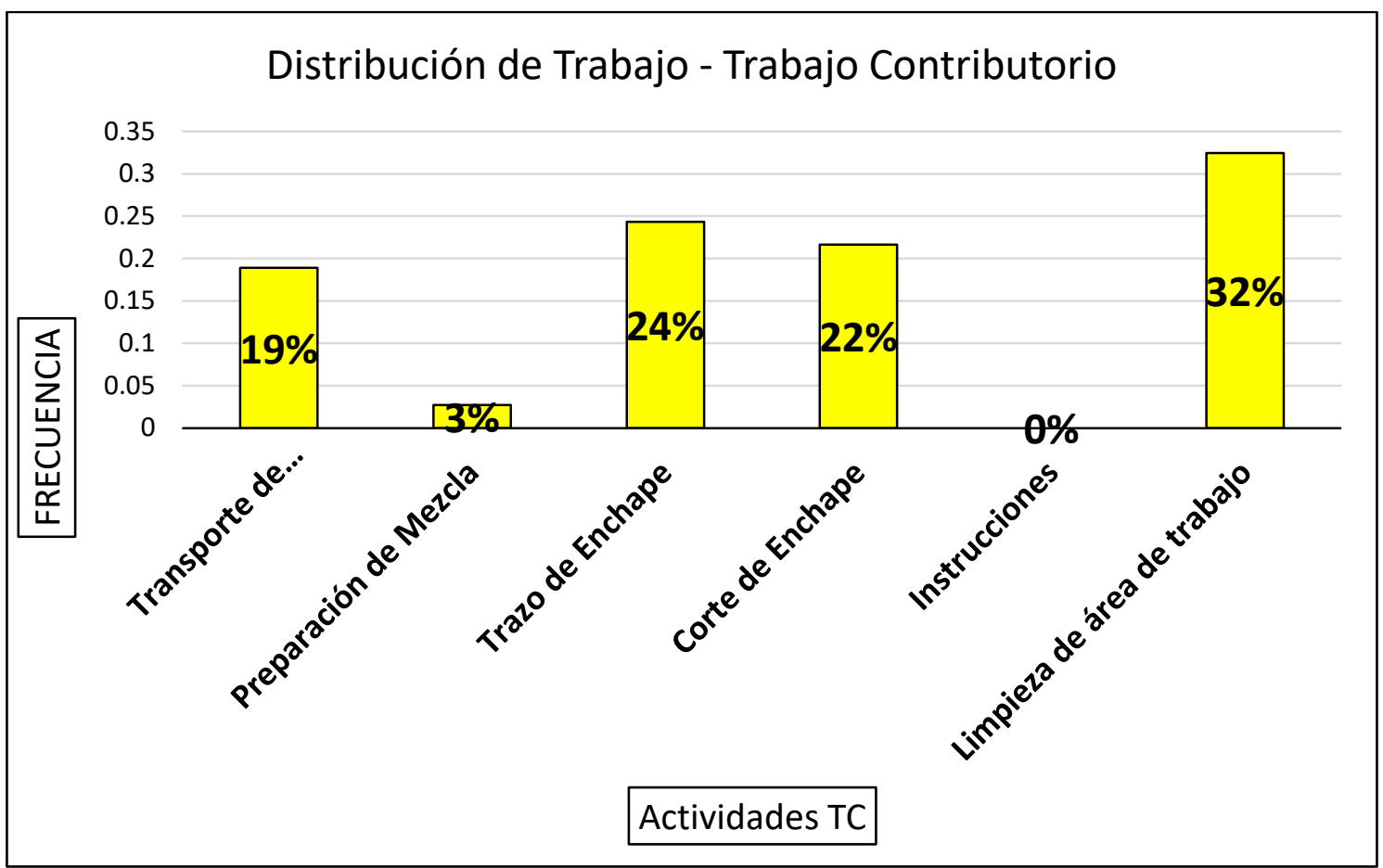

Fuente: Elaboración Propia.

Figura $N^{\circ}$ 94: Distribución de Trabajo No Contributorio - Carta Balance ${ }^{\circ} 11$

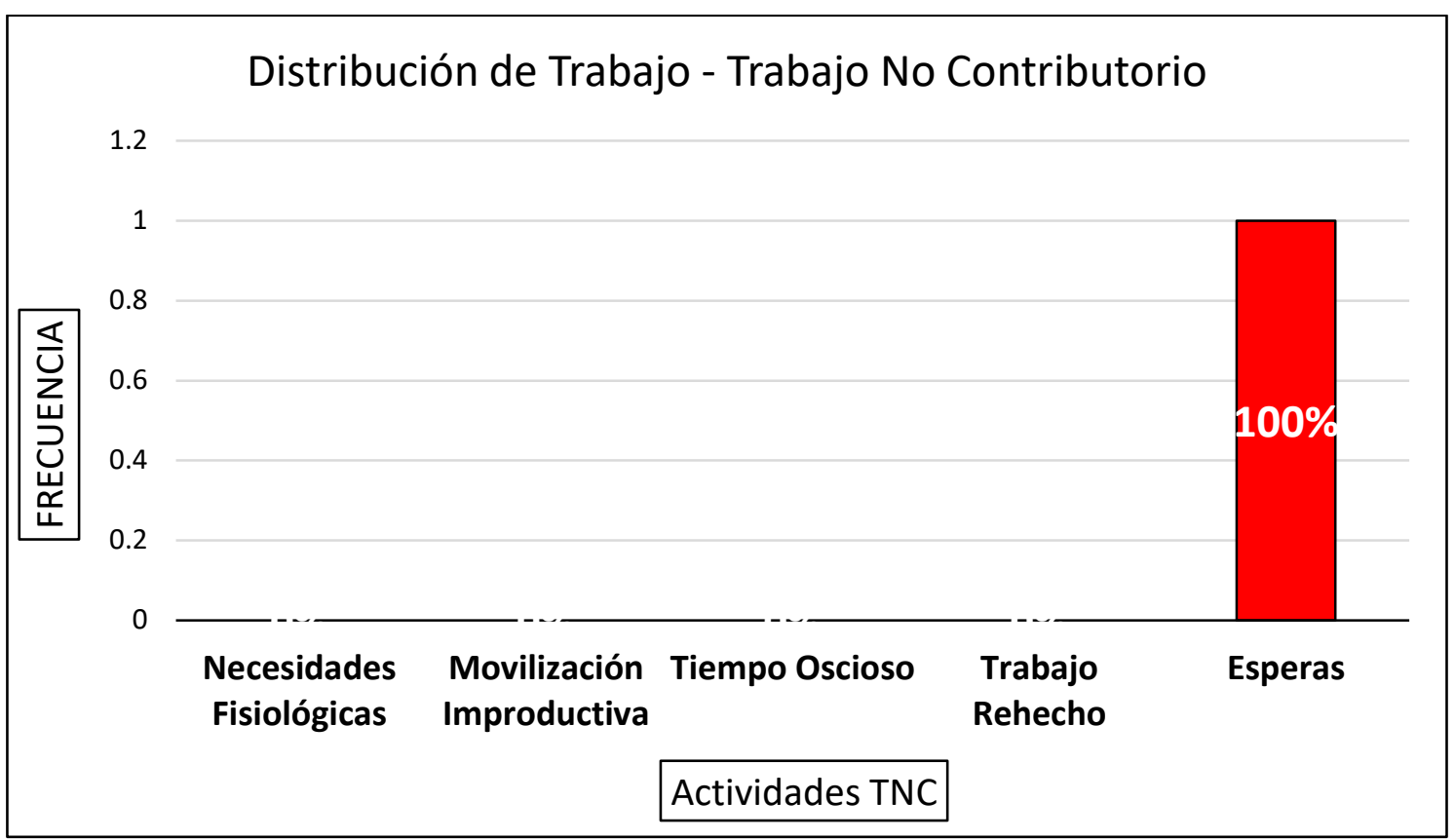

Fuente: Elaboración Propia. 
Tabla N² 27: Carta Balance ${ }^{\circ} 12$ - Enchape de Cerámico

\begin{tabular}{|c|c|c|c|c|c|c|c|c|}
\hline $\mathrm{N}^{*}$ & Obrero \#1 & Obrero \#2 & 31 & TE & $\mathrm{L}$ & 61 & $A P$ & $E$ \\
\hline 1 & $\mathrm{COE}$ & $E$ & 32 & $C P$ & $\mathrm{~L}$ & 62 & $C C$ & E \\
\hline 2 & TE & $E$ & 33 & $\mathrm{CE}$ & $\mathrm{L}$ & 63 & NE & $\mathrm{E}$ \\
\hline 3 & TE & $E$ & 34 & $A P$ & $t$ & 64 & TE & $E$ \\
\hline 4 & TM & $E$ & 35 & $\mathrm{CC}$ & $E$ & 65 & $\mathrm{TE}$ & $E$ \\
\hline 5 & $\mathrm{TM}$ & E & 36 & $C P$ & $E$ & 66 & COE & E \\
\hline 6 & TM & E & 37 & $\mathrm{CP}$ & E & 67 & COE & E \\
\hline 7 & TM & $E$ & 38 & $\mathrm{CE}$ & $E$ & 68 & $\mathrm{COE}$ & E \\
\hline 8 & $B M$ & $E$ & 39 & $\mathrm{CE}$ & $\mathrm{E}$ & 69 & $C P$ & $\mathrm{E}$ \\
\hline 9 & $B M$ & $E$ & 40 & $A P$ & $E$ & 70 & CE & $\varepsilon$ \\
\hline 10 & $\mathrm{TE}$ & $L$ & 41 & $A P$ & $E$ & 71 & $A P$ & $E$ \\
\hline 11 & $\mathrm{TE}$ & $L$ & 42 & NE & $E$ & 72 & $C C$ & $E$ \\
\hline 12 & TE & L & 43 & BM & E & 73 & $C C$ & E \\
\hline 13 & $\mathrm{COE}$ & $\mathrm{L}$ & 44 & MI & $E$ & 74 & TE & $E$ \\
\hline 14 & $\mathrm{COE}$ & $E$ & 45 & $C P$ & $\mathrm{E}$ & 75 & $\mathrm{COE}$ & $E$ \\
\hline 15 & $\cot$ & L & 46 & $A P$ & $E$ & 76 & $\mathrm{COE}$ & $\varepsilon$ \\
\hline 16 & $\mathrm{TE}$ & $L$ & 47 & $\mathrm{CC}$ & $E$ & $n$ & $C P$ & $E$ \\
\hline 17 & $C P$ & E & 48 & TE & $E$ & 78 & $C E$ & $E$ \\
\hline 18 & $C C$ & E & 49 & COE & E & 79 & AP & E \\
\hline 19 & $\mathrm{NE}$ & $E$ & 50 & AP & E & 80 & CE & $E$ \\
\hline 20 & $\mathrm{NE}$ & $E$ & 51 & $A P$ & $\mathrm{E}$ & 81 & TE & $\mathrm{E}$ \\
\hline 21 & NE & $E$ & 52 & $A P$ & $E$ & 82 & $A P$ & $E$ \\
\hline 22 & $\mathrm{CC}$ & $E$ & 53 & $\mathrm{NE}$ & E & 83 & AP & $E$ \\
\hline 23 & MI & E & 54 & $\mathrm{CC}$ & E & 84 & $C C$ & E \\
\hline 24 & MI & E & 55 & TE & E & 85 & $\mathrm{~L}$ & E \\
\hline 25 & TE & E & 56 & $\mathrm{NE}$ & $E$ & 86 & $\mathrm{COE}$ & $E$ \\
\hline 26 & $\mathrm{MI}$ & $E$ & 57 & TE & $E$ & 87 & CE & $\mathrm{E}$ \\
\hline 27 & $\mathrm{MI}$ & $t$ & 58 & MI & $E$ & 88 & $C C$ & $E$ \\
\hline 28 & MI & $L$ & 59 & COE & L & 89 & MI & $E$ \\
\hline 29 & $\mathrm{COE}$ & L & 60 & $C P$ & E & 90 & 1 & $E$ \\
\hline 30 & $\mathrm{COE}$ & L & & & & & & \\
\hline
\end{tabular}

Fuente: Elaboración Propia. 
Figura Nº 95: Distribución de Trabajo de Enchape de Cerámico - Carta Balance ${ }^{\circ} 12$

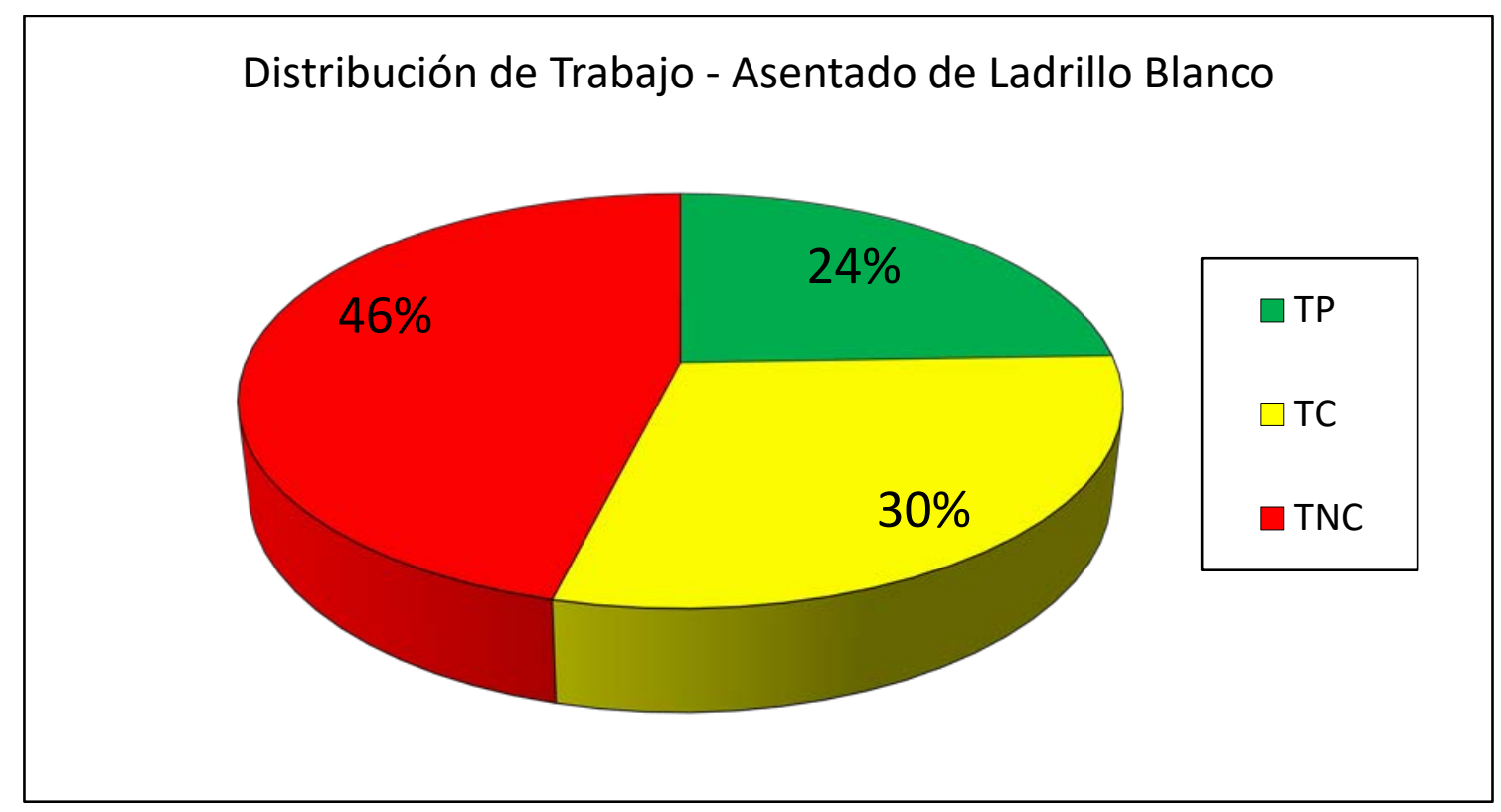

Fuente: Elaboración Propia.

Figura No ${ }^{\circ}$ 96: Distribución de Trabajo Productivo - Carta Balance ${ }^{\circ} 12$

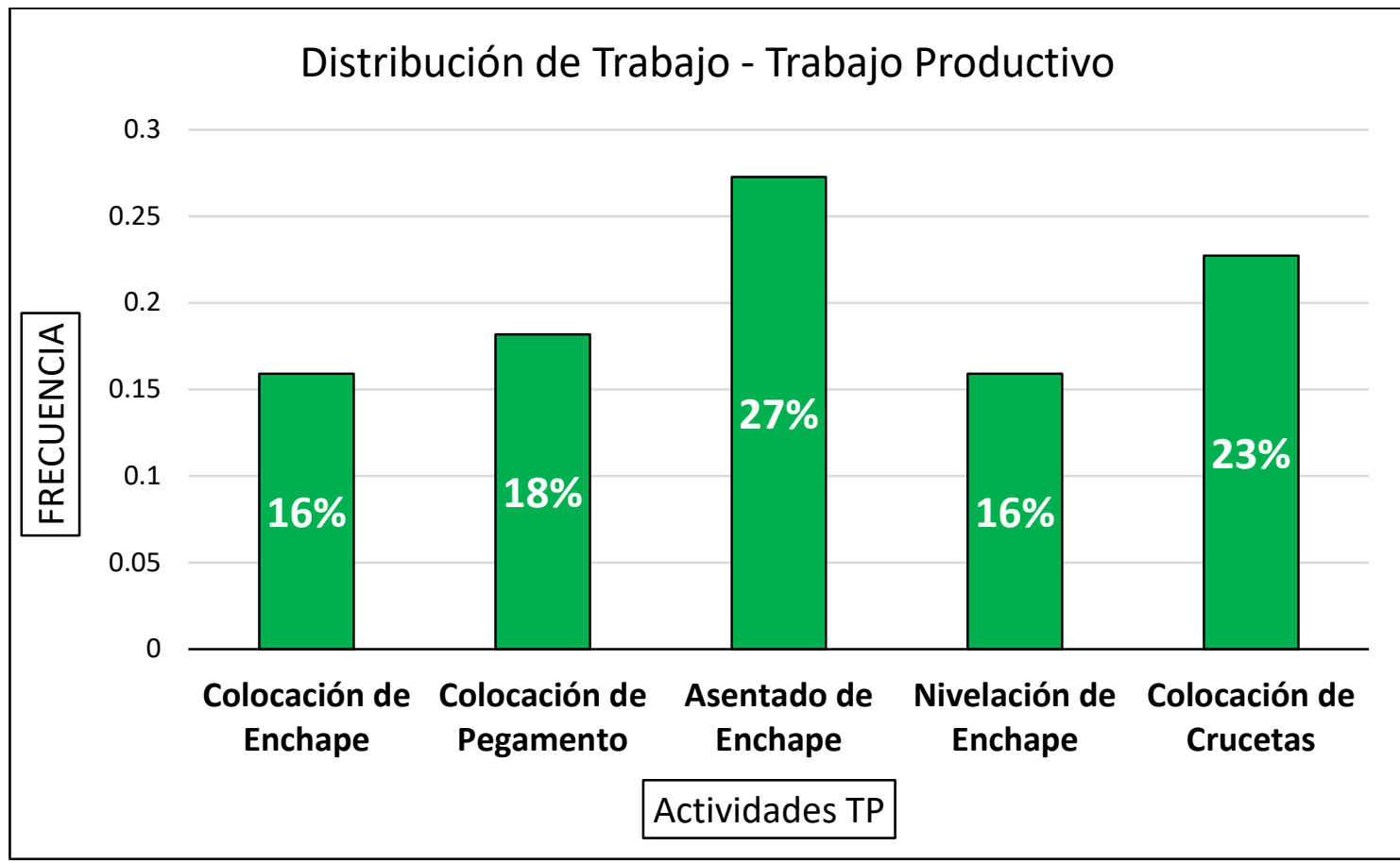

Fuente: Elaboración Propia. 
Figura Nº 97: Distribución de Trabajo Contributorio - Carta Balance ${ }^{\circ} 12$

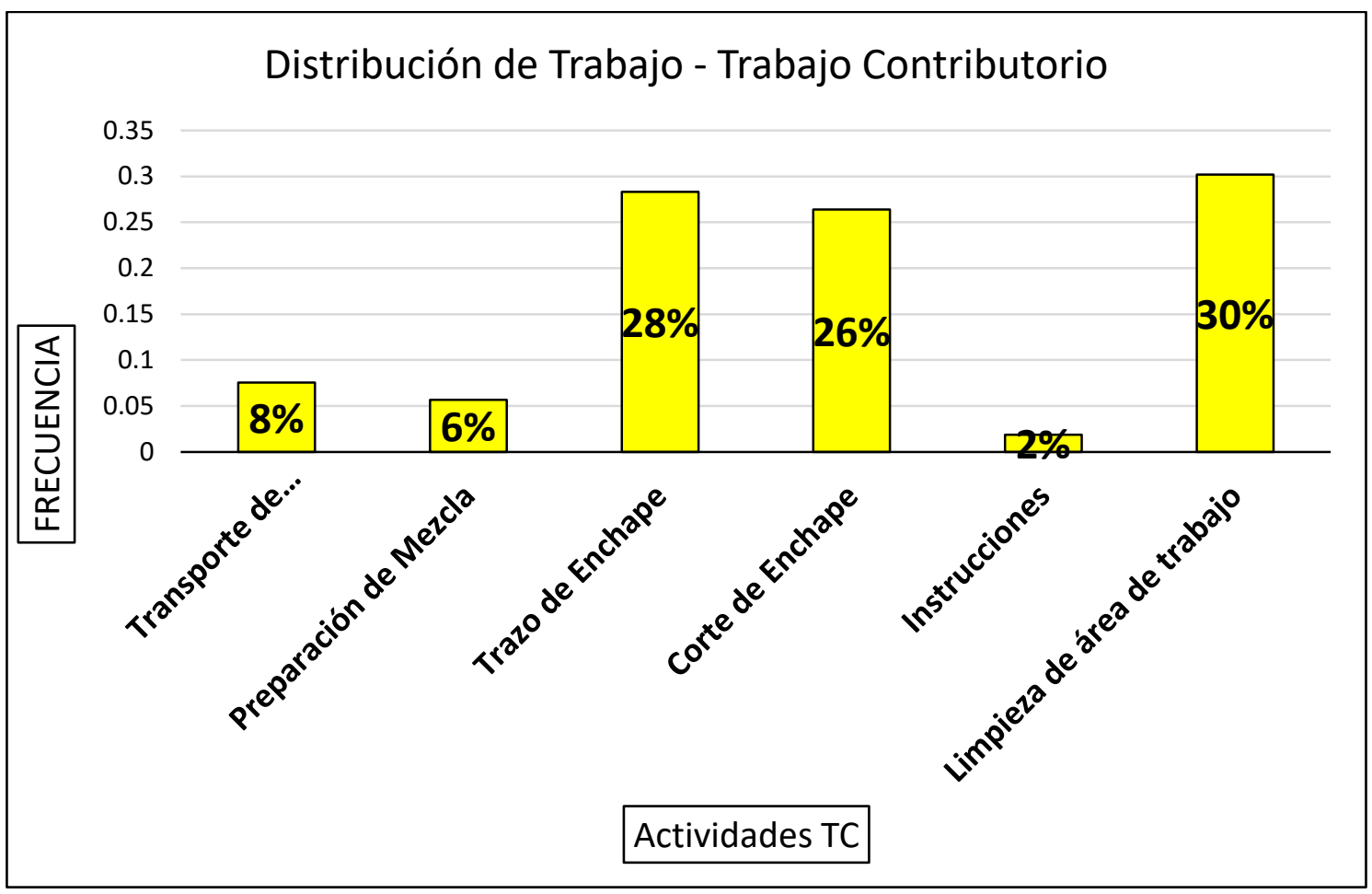

Fuente: Elaboración Propia.

Figura Nº 98:Distribución de Trabajo No Contributorio - Carta Balance ${ }^{\circ} 12$

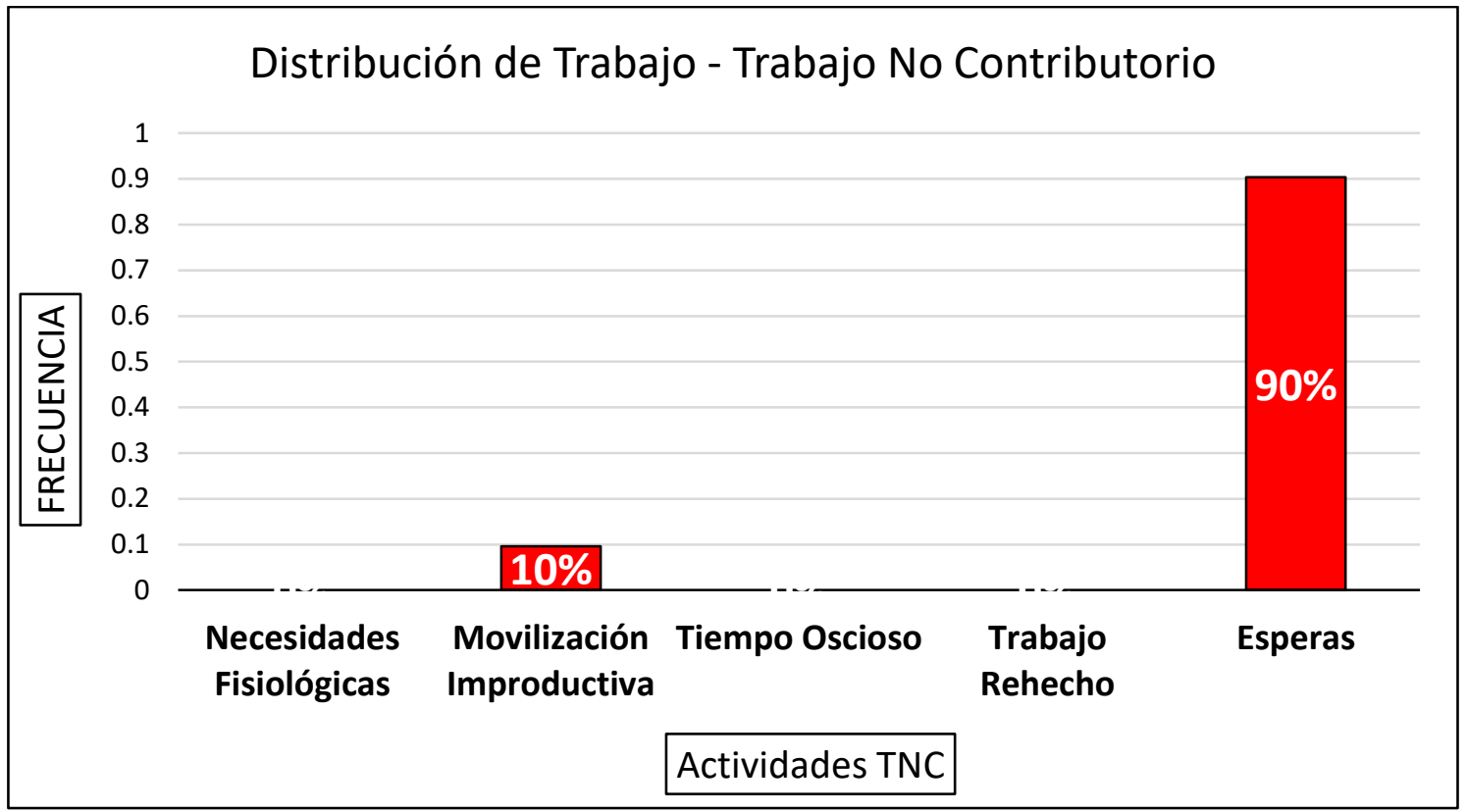

Fuente: Elaboración Propia. 
Tabla N² 28: Carta Balance ${ }^{\circ} 13$ - Enchape de Cerámico

\begin{tabular}{|c|c|c|}
\hline $\mathrm{N}^{*}$ & Obrero $\| 1$ & Obrero \\
\hline 1 & $\operatorname{COE}$ & $E$ \\
\hline 2 & TE & $E$ \\
\hline 3 & TE & E \\
\hline 4 & $\mathrm{TM}$ & E \\
\hline 5 & $\mathrm{TM}$ & $E$ \\
\hline 6 & $\mathrm{TM}$ & $E$ \\
\hline 7 & $\mathrm{TM}$ & $E$ \\
\hline 8 & $B M$ & E \\
\hline 9 & $B M$ & E \\
\hline 10 & TE & L \\
\hline 11 & TE & L \\
\hline 12 & TE & L \\
\hline 13 & $\mathrm{COE}$ & L \\
\hline 14 & COE & $E$ \\
\hline 15 & $\mathrm{COE}$ & $\mathrm{L}$ \\
\hline 16 & TE & $\mathrm{L}$ \\
\hline 17 & $C P$ & E \\
\hline 18 & $\mathrm{CC}$ & E \\
\hline 19 & NE & $E$ \\
\hline 20 & NE & $E$ \\
\hline 21 & NE & $E$ \\
\hline 22 & $C C$ & E \\
\hline 23 & MI & E \\
\hline 24 & MI & $E$ \\
\hline 25 & TE & $E$ \\
\hline 26 & MI & E \\
\hline 27 & MI & L \\
\hline 28 & MI & L \\
\hline 29 & $\cot$ & $\mathrm{l}$ \\
\hline 30 & $\operatorname{COE}$ & L \\
\hline
\end{tabular}

\begin{tabular}{|c|c|c|}
\hline 31 & TE & $\mathrm{L}$ \\
\hline 32 & $C P$ & L \\
\hline 33 & CE & L \\
\hline 34 & AP & L \\
\hline 35 & $\mathrm{CC}$ & E \\
\hline 36 & $C P$ & E \\
\hline 37 & $C P$ & $E$ \\
\hline 38 & $\mathrm{CE}$ & E \\
\hline 39 & $\mathrm{CE}$ & E \\
\hline 40 & AP & $\mathbf{E}$ \\
\hline 41 & AP & $E$ \\
\hline 42 & NE & $E$ \\
\hline 43 & BM & E \\
\hline 44 & MI & E \\
\hline 45 & $C P$ & E \\
\hline 46 & AP & $E$ \\
\hline 47 & $C C$ & $\mathrm{E}$ \\
\hline 48 & TE & E \\
\hline 49 & COE & $\mathbf{E}$ \\
\hline 50 & AP & $E$ \\
\hline 51 & AP & $E$ \\
\hline 52 & AP & $E$ \\
\hline 53 & NE & E \\
\hline 54 & $C C$ & E \\
\hline 55 & TE & $\mathrm{E}$ \\
\hline 56 & NE & $\mathrm{E}$ \\
\hline 57 & TE & E \\
\hline 58 & MI & $E$ \\
\hline 59 & COE & L \\
\hline 60 & $C P$ & $E$ \\
\hline
\end{tabular}

\begin{tabular}{|c|c|c|}
\hline 61 & AP & $E$ \\
\hline 62 & $\mathrm{CC}$ & $E$ \\
\hline 63 & NE & $\mathrm{E}$ \\
\hline 64 & TE & $E$ \\
\hline 65 & TE & E \\
\hline 66 & COE & $E$ \\
\hline 67 & $\operatorname{COE}$ & $E$ \\
\hline 68 & $\mathrm{COE}$ & E \\
\hline 69 & $C P$ & E \\
\hline 70 & CE & E \\
\hline 71 & AP & $E$ \\
\hline 72 & $\mathrm{CC}$ & $\mathrm{E}$ \\
\hline 73 & $\mathrm{CC}$ & E \\
\hline 74 & $\mathrm{TE}$ & E \\
\hline 75 & COE & $E$ \\
\hline 76 & $\operatorname{COE}$ & $E$ \\
\hline 77 & $C P$ & $\mathrm{E}$ \\
\hline 78 & CE & E \\
\hline 79 & AP & E \\
\hline 80 & $C E$ & $E$ \\
\hline 81 & TE & $E$ \\
\hline 82 & AP & $\mathrm{E}$ \\
\hline 83 & AP & E \\
\hline 84 & $\mathrm{CC}$ & E \\
\hline 85 & $\mathrm{~L}$ & $E$ \\
\hline 86 & $\mathrm{COE}$ & $\mathrm{E}$ \\
\hline 87 & $\mathrm{CE}$ & $E$ \\
\hline 88 & $\mathrm{CC}$ & E \\
\hline 89 & MI & $E$ \\
\hline 90 & 1 & $E$ \\
\hline
\end{tabular}

Fuente: Elaboración Propia. 
Figura Nº 99: Distribución de Trabajo de Enchape de Cerámico - Carta Balance ${ }^{\circ} 13$

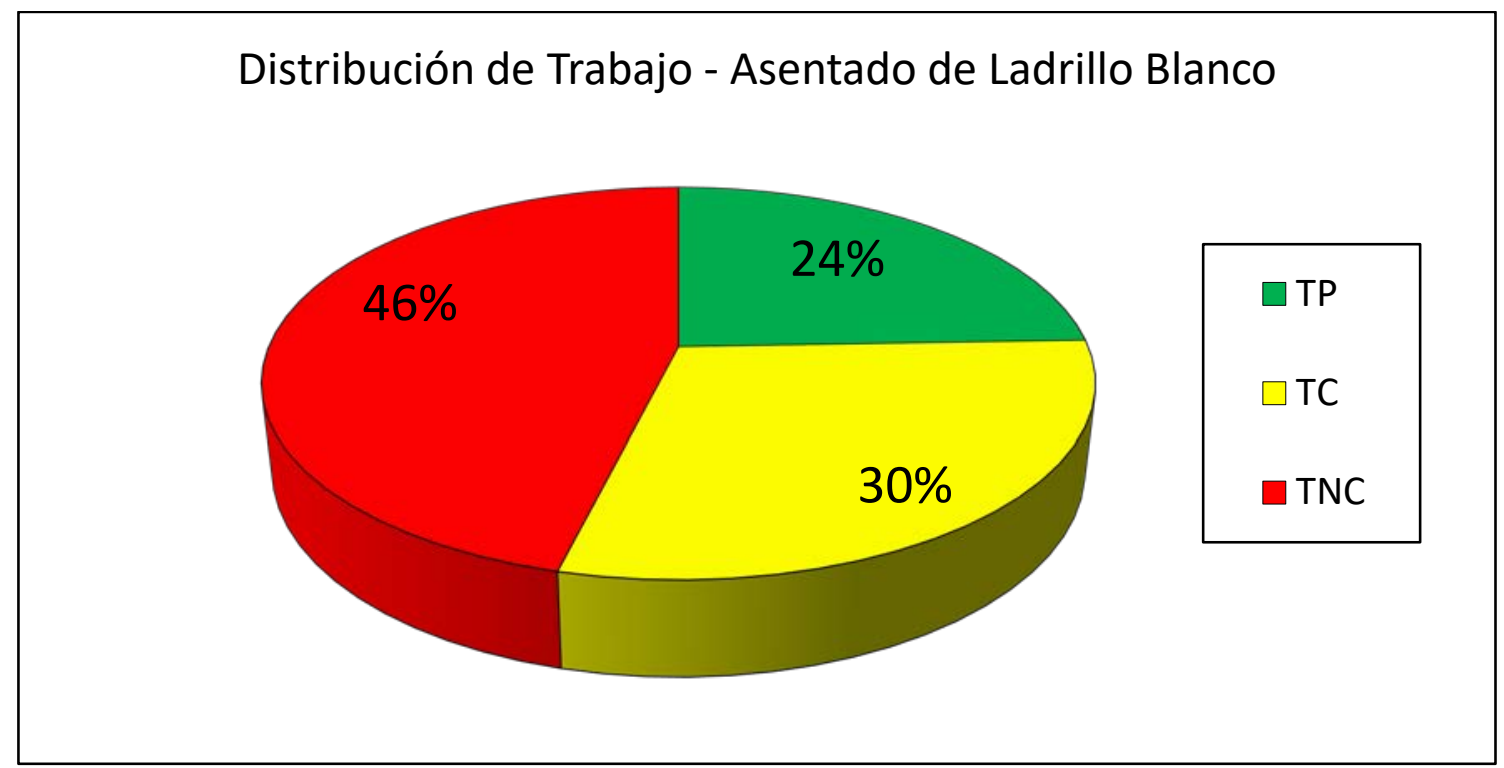

Fuente: Elaboración Propia.

Figura $N^{\circ}$ 100: Distribución de Trabajo Productivo - Carta Balance ${ }^{\circ} 13$

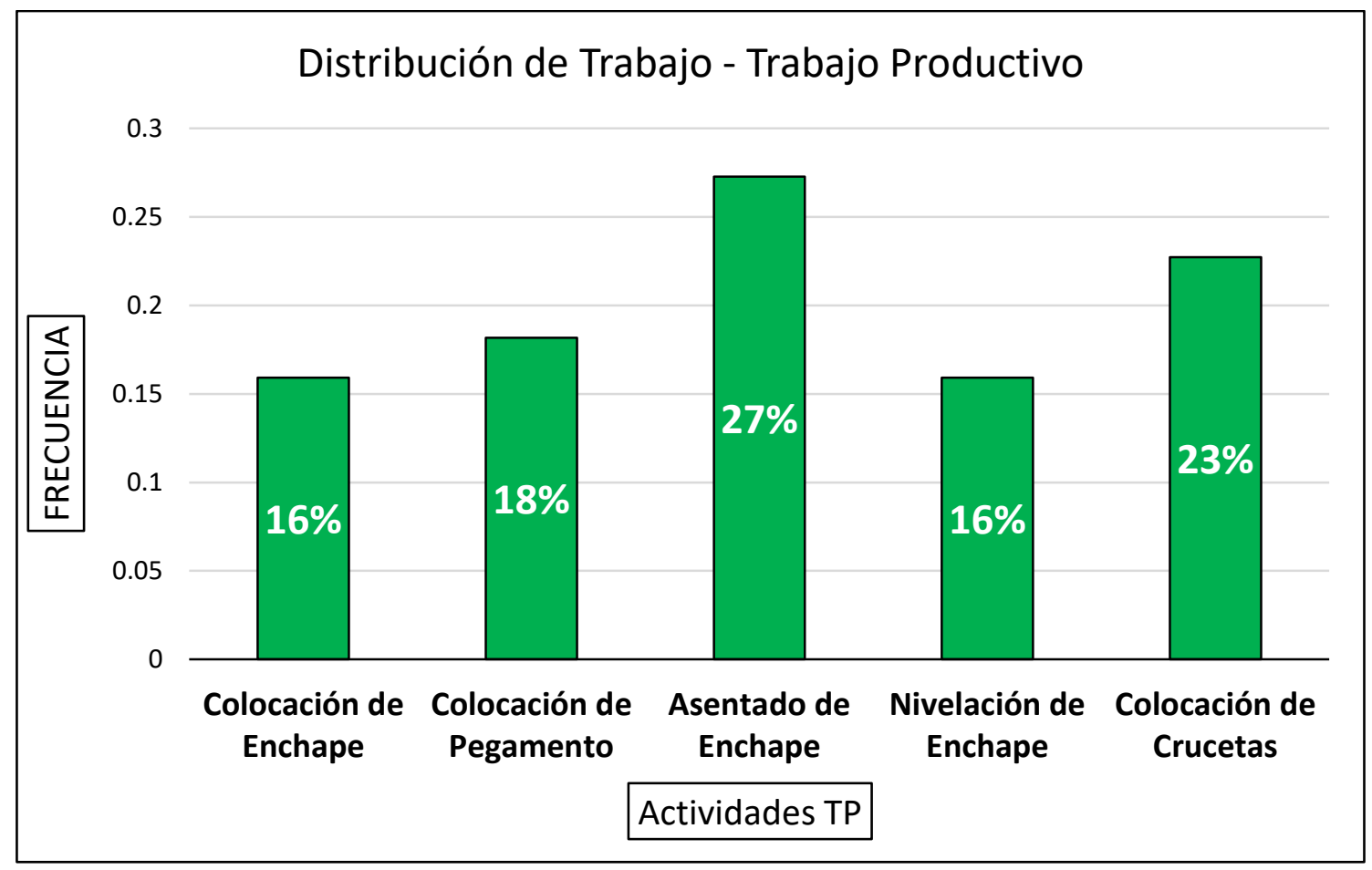

Fuente: Elaboración Propia. 
Figura $N^{\circ} 101$ : Distribución de Trabajo Contributorio - Carta Balance ${ }^{\circ} 13$

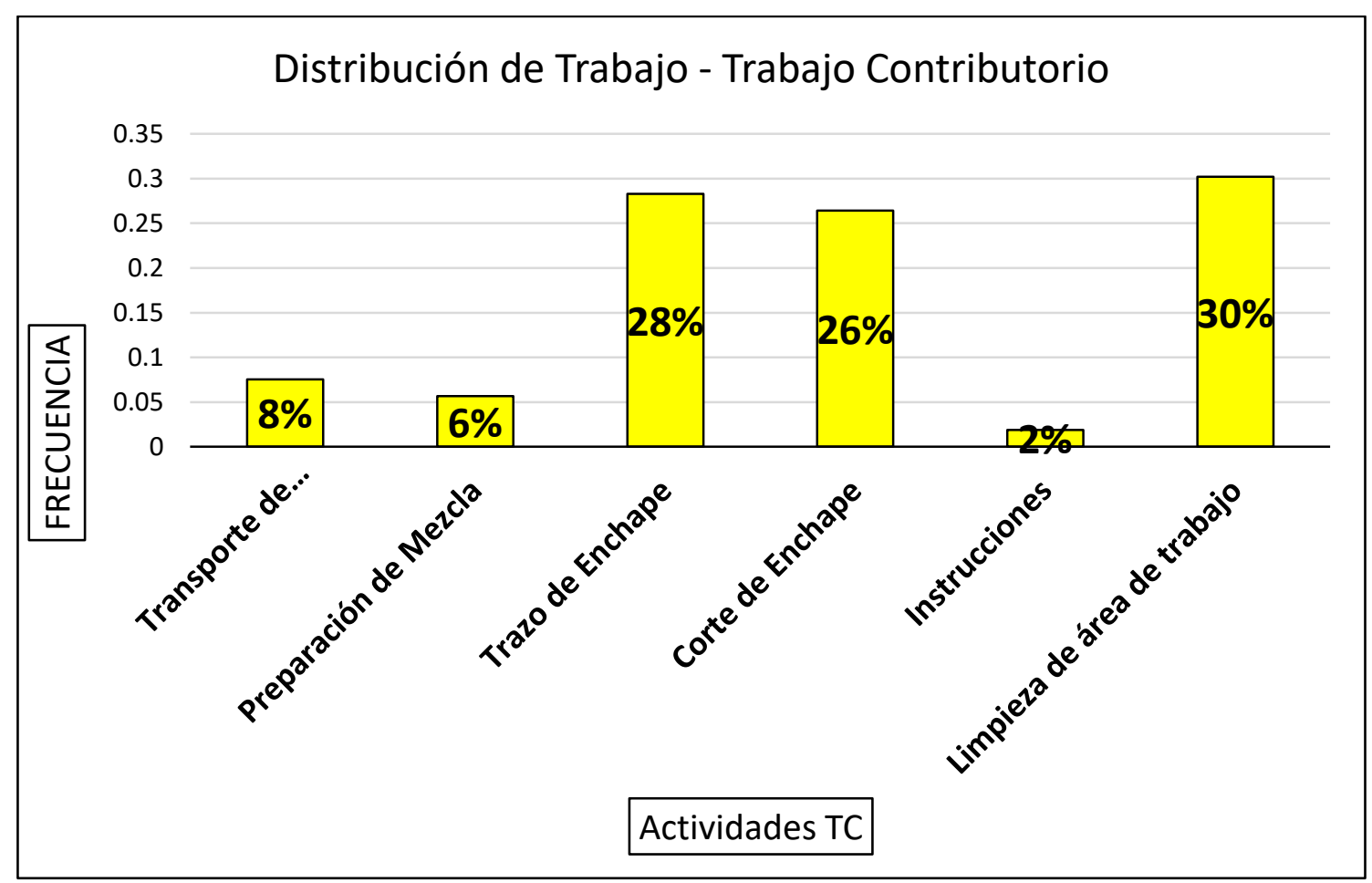

Fuente: Elaboración Propia.

Figura N ${ }^{\circ}$ 102: Distribución de Trabajo No Contributorio - Carta Balance ${ }^{\circ} 13$

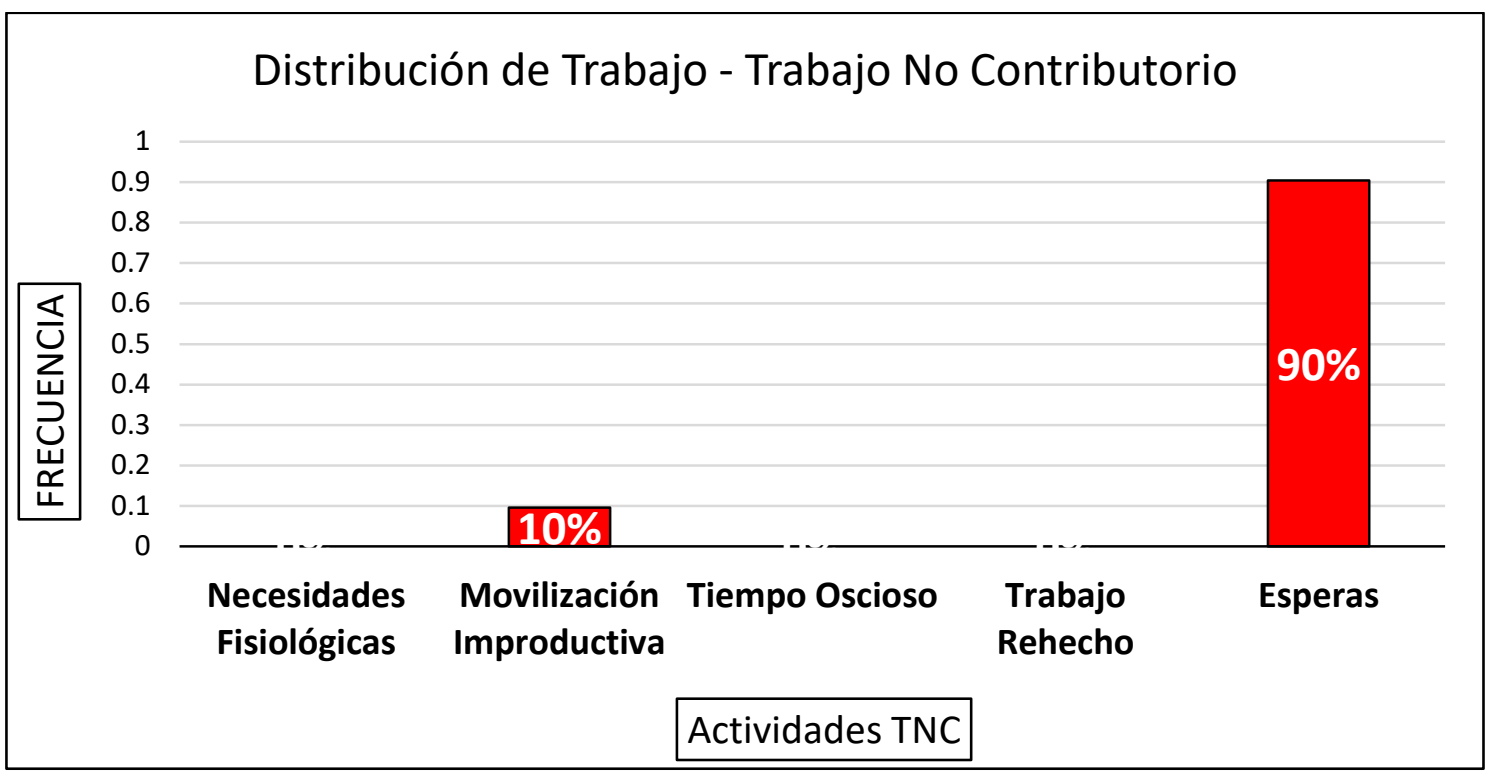

Fuente: Elaboración Propia. 
Tabla N² 29: Carta Balance ${ }^{\circ} 14$ - Enchape de Cerámico

\begin{tabular}{|c|c|c|c|c|c|c|c|c|}
\hline $\mathrm{N}^{*}$ & Obrero $\| 1$ & Obrero 112 & 31 & $C C$ & TO & 61 & L & l \\
\hline 1 & $\operatorname{COE}$ & $E$ & 32 & 1 & $\mathrm{l}$ & 62 & $\mathrm{CE}$ & L \\
\hline 2 & $\mathrm{NE}$ & E & 33 & $\mathrm{~L}$ & $E$ & 63 & AP & L \\
\hline 3 & $C P$ & E & 34 & $\mathrm{TM}$ & E & 64 & L & L \\
\hline 4 & $C C$ & E & 35 & $\mathrm{COE}$ & $E$ & 65 & $\operatorname{COE}$ & L \\
\hline 5 & $A P$ & $E$ & 36 & $\mathrm{COE}$ & $E$ & 66 & $A P$ & L \\
\hline 6 & $A P$ & $\mathrm{E}$ & 37 & $C P$ & $E$ & 67 & L & $\mathrm{L}$ \\
\hline 7 & L & E & 38 & AP & L & 68 & L & L \\
\hline 8 & $C P$ & E & 39 & L & L & 69 & L & L \\
\hline 9 & $\mathrm{CC}$ & $E$ & 40 & TE & $\mathrm{L}$ & 70 & L & L \\
\hline 10 & $A P$ & $E$ & 41 & TE & E & 71 & L & $\mathrm{E}$ \\
\hline 11 & TE & $E$ & 42 & TE & E & 72 & L & E \\
\hline 12 & CE & E & 43 & COE & E & 73 & $L$ & E \\
\hline 13 & $A P$ & $E$ & 44 & $A P$ & $E$ & 74 & 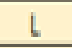 & $E$ \\
\hline 14 & TE & $\mathrm{TM}$ & 45 & NE & $E$ & 75 & $\mathrm{TM}$ & $\mathrm{TM}$ \\
\hline 15 & NE & $\mathrm{TM}$ & 46 & $C C$ & E & 76 & L & $\mathrm{TM}$ \\
\hline 16 & $C C$ & $\mathrm{TM}$ & 47 & L & E & 77 & L & $\mathrm{TM}$ \\
\hline 17 & TE & $\mathrm{TM}$ & 48 & TE & $E$ & 78 & L & $\mathrm{TM}$ \\
\hline 18 & TE & $\mathrm{TM}$ & 49 & TE & $E$ & 79 & $\mathrm{~L}$ & $\mathrm{TM}$ \\
\hline 19 & COE & $\mathrm{TM}$ & 50 & TE & E & 80 & COE & $\mathrm{TM}$ \\
\hline 20 & COE & $\mathrm{TM}$ & 51 & COE & E & 81 & COE & TO \\
\hline 21 & $\mathrm{CC}$ & $\mathrm{TM}$ & 52 & $\mathrm{COE}$ & $E$ & 82 & $\mathrm{TM}$ & TO \\
\hline 22 & TE & $E$ & 53 & $C P$ & $E$ & 83 & $\mathrm{BM}$ & $\mathrm{TM}$ \\
\hline 23 & NE & $E$ & 54 & $C P$ & E & 84 & $B M$ & E \\
\hline 24 & NE & $\mathrm{TM}$ & 55 & $C P$ & E & 85 & $\mathrm{TM}$ & $E$ \\
\hline 25 & NE & $\mathrm{TM}$ & 56 & $A P$ & $E$ & 86 & $\cot$ & $\mathrm{TM}$ \\
\hline 26 & $\mathrm{NE}$ & $\mathrm{TM}$ & 57 & $C C$ & E & 87 & $C P$ & $\mathrm{TM}$ \\
\hline 27 & NE & $\mathrm{TM}$ & 58 & $\mathrm{COE}$ & $E$ & 88 & CP & $E$ \\
\hline 28 & 1 & TO & 59 & AP & L & 89 & $A P$ & $\mathrm{TM}$ \\
\hline 29 & 1 & $\mathrm{TM}$ & 60 & TE & $t$ & 90 & NE & $E$ \\
\hline 30 & $C P$ & TO & & & & & & \\
\hline
\end{tabular}

Fuente: Elaboración Propia. 
Figura Nº 103: Distribución de Trabajo de Enchape de Cerámico - Carta Balance 14

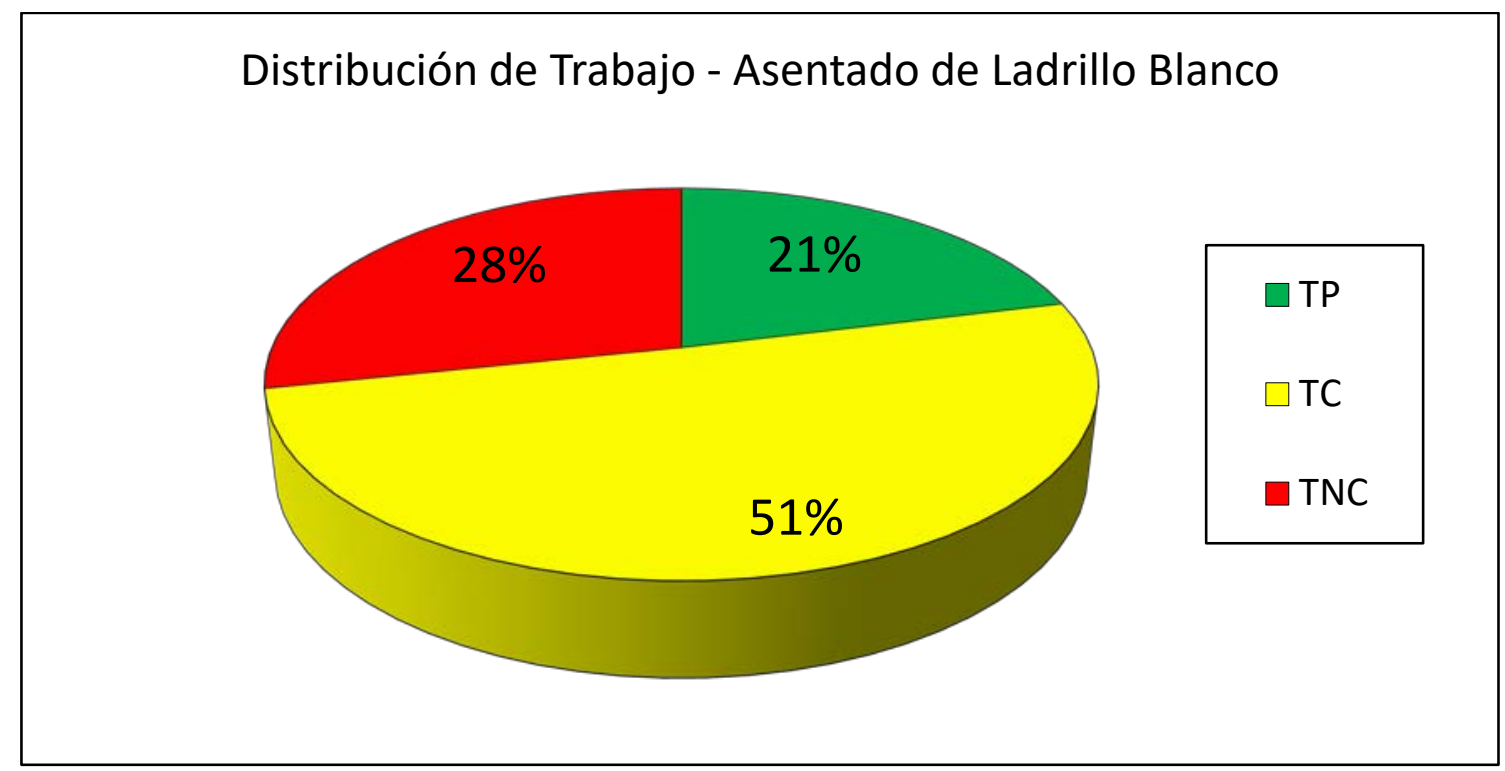

Fuente: Elaboración Propia.

Figura $N^{\circ}$ 104: Distribución de Trabajo Productivo - Carta Balance ${ }^{\circ} 14$

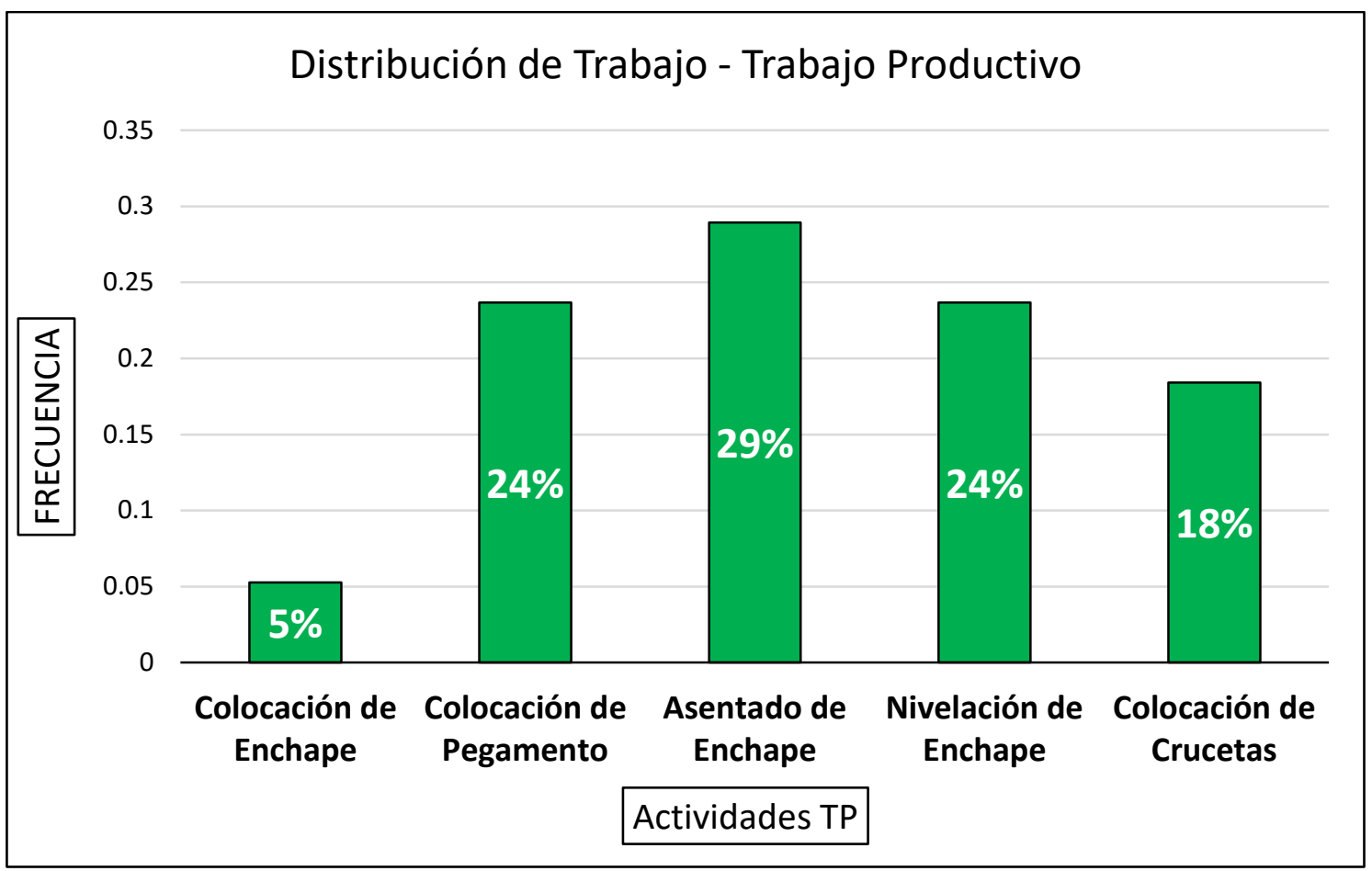

Fuente: Elaboración Propia. 
Figura $N^{\circ} 105$ : Distribución de Trabajo Contributorio - Carta Balance ${ }^{\circ} 14$

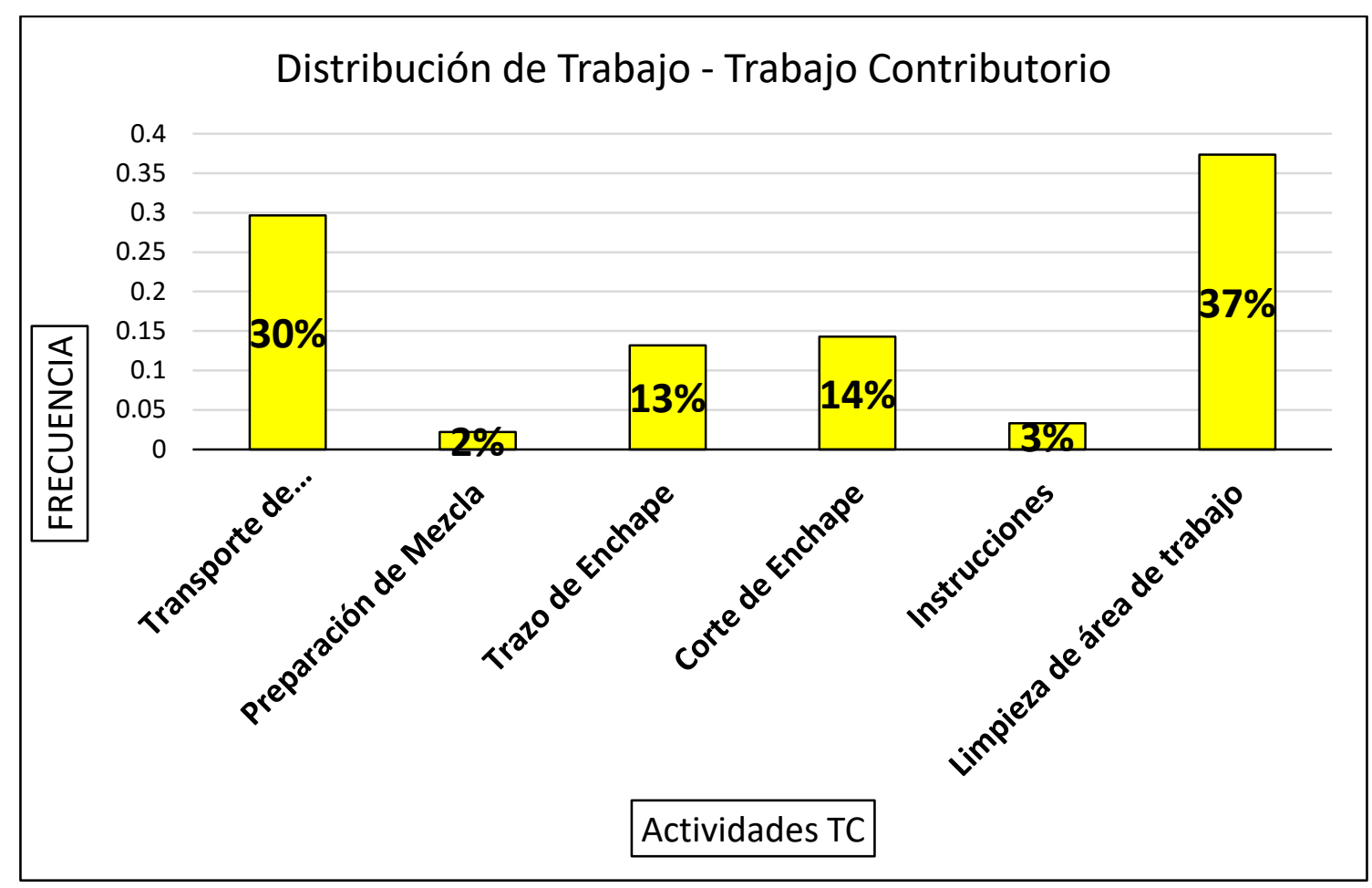

Fuente: Elaboración Propia.

Figura N ${ }^{\circ}$ 106: Distribución de Trabajo No Contributorio - Carta Balance ${ }^{\circ} 14$

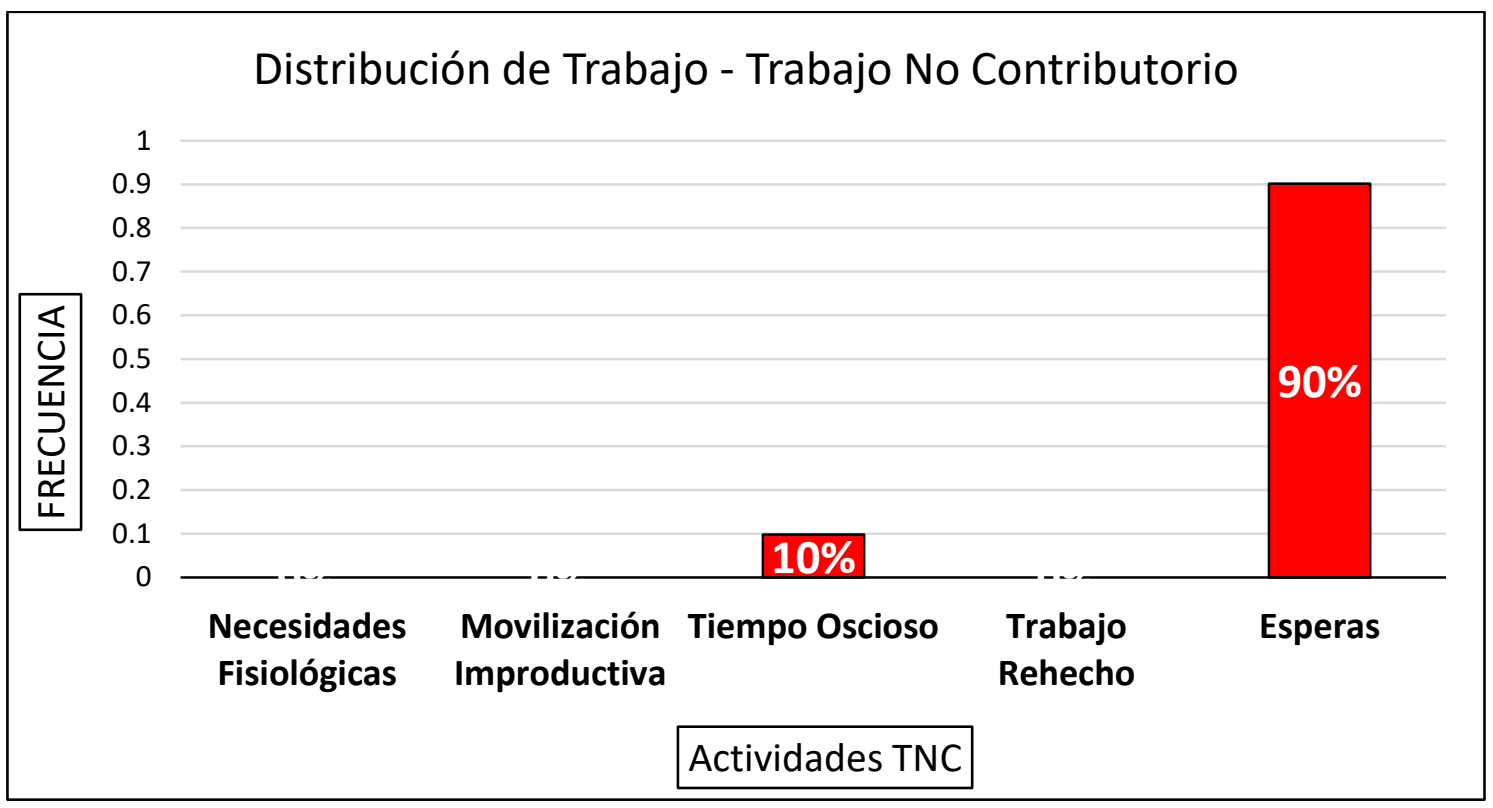

Fuente: Elaboración Propia. 
Tabla N 30: Carta Balance ${ }^{\circ} 15$ - Enchape de Cerámico

\begin{tabular}{|c|c|c|c|c|c|c|c|c|}
\hline $\mathrm{N}^{*}$ & Obrero \#1 & Obrero $\mathrm{H2}$ & 31 & $\mathrm{CC}$ & $E$ & 61 & $\mathrm{TM}$ & TM \\
\hline 1 & BM & E & 32 & $c C$ & $E$ & 62 & $\mathrm{TM}$ & TM \\
\hline 2 & $B M$ & E & 33 & $C C$ & $E$ & 63 & $\mathrm{TM}$ & TM \\
\hline 3 & BM & E & 34 & $L$ & $E$ & 64 & $\mathrm{TM}$ & $\mathrm{TM}$ \\
\hline 4 & L & $E$ & 35 & BM & TO & 65 & $\mathrm{TM}$ & TM \\
\hline 5 & $C P$ & E & 36 & $B M$ & TO & 66 & TM & $\mathrm{TM}$ \\
\hline 6 & $C E$ & TO & 37 & $B M$ & TO & 67 & L & L \\
\hline 7 & CE & TO & 38 & $\mathrm{BM}$ & TO & 68 & L & L \\
\hline 8 & CE & TO & 39 & $C P$ & TO & 69 & $C P$ & L \\
\hline 9 & $C C$ & TO & 40 & $\mathrm{CP}$ & TO & 70 & $\mathrm{CP}$ & $L$ \\
\hline 10 & $\mathrm{CC}$ & TO & 41 & $C P$ & TO & 71 & $\mathrm{CE}$ & L \\
\hline 11 & $\mathrm{CC}$ & TO & 42 & COE & TO & 72 & CE & TM \\
\hline 12 & $\mathrm{CC}$ & TO & 43 & $\mathrm{CE}$ & TO & 73 & $\mathrm{CE}$ & TM \\
\hline 13 & $C P$ & $E$ & 44 & CE & TO & 74 & CE & $\mathrm{TM}$ \\
\hline 14 & $C P$ & $E$ & 45 & $\mathrm{CE}$ & TO & 75 & $C P$ & $\mathrm{TM}$ \\
\hline 15 & CE & E & 46 & $\mathrm{CE}$ & TO & 76 & $\mathrm{CE}$ & E \\
\hline 16 & CE & E & 47 & CE & TO & 77 & L & E \\
\hline 17 & $\mathrm{CE}$ & E & 48 & $L$ & TO & 78 & $\mathrm{CC}$ & $\mathrm{TM}$ \\
\hline 18 & CE & $E$ & 49 & $C P$ & TO & 79 & $\mathrm{CC}$ & TM \\
\hline 19 & L & $L$ & 50 & $\mathrm{CE}$ & TO & 80 & $\mathrm{CC}$ & $L$ \\
\hline 20 & $C C$ & $L$ & 51 & $C C$ & TO & 81 & $\mathrm{CC}$ & L \\
\hline 21 & $\mathrm{CC}$ & L & 52 & $C C$ & TO & 82 & $\mathrm{CC}$ & L \\
\hline 22 & $C C$ & L & 53 & $C C$ & TO & 83 & $C P$ & L \\
\hline 23 & $C P$ & E & 54 & $C C$ & TO & 84 & $C P$ & $\mathrm{TM}$ \\
\hline 24 & L & E & 55 & $C C$ & TO & 85 & CE & $\mathrm{TM}$ \\
\hline 25 & $C P$ & $E$ & 56 & L & TO & 86 & $C E$ & $\mathrm{TM}$ \\
\hline 26 & CE & E & 57 & 1 & 1 & 87 & $\mathrm{NE}$ & $E$ \\
\hline 27 & CE & $\mathbf{E}$ & 58 & 1 & 1 & 88 & $\mathrm{NE}$ & $E$ \\
\hline 28 & CE & $E$ & 59 & $\mathrm{TM}$ & $\mathrm{TM}$ & 89 & $\mathrm{CE}$ & $E$ \\
\hline 29 & CE & $E$ & 60 & $\mathrm{TM}$ & $\mathrm{TM}$ & 90 & $\mathrm{CE}$ & $E$ \\
\hline 30 & CE & $E$ & & & & & & \\
\hline
\end{tabular}

Fuente: Elaboración Propia. 
Figura Nº 107: Distribución de Trabajo de Enchape de Cerámico - Carta Balance ${ }^{\circ} 15$

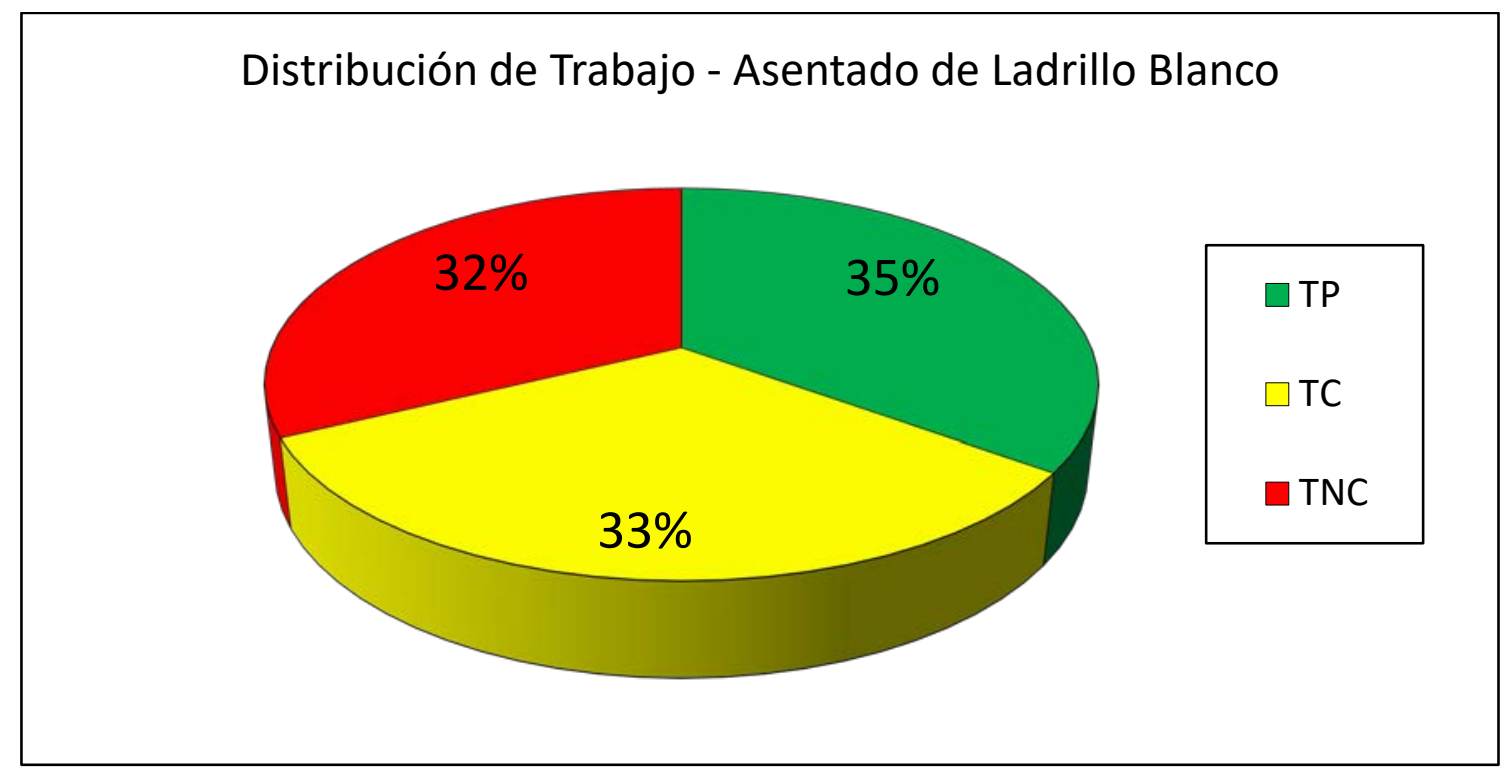

Fuente: Elaboración Propia.

Figura $N^{\circ}$ 108: Distribución de Trabajo Productivo - Carta Balance ${ }^{\circ} 15$

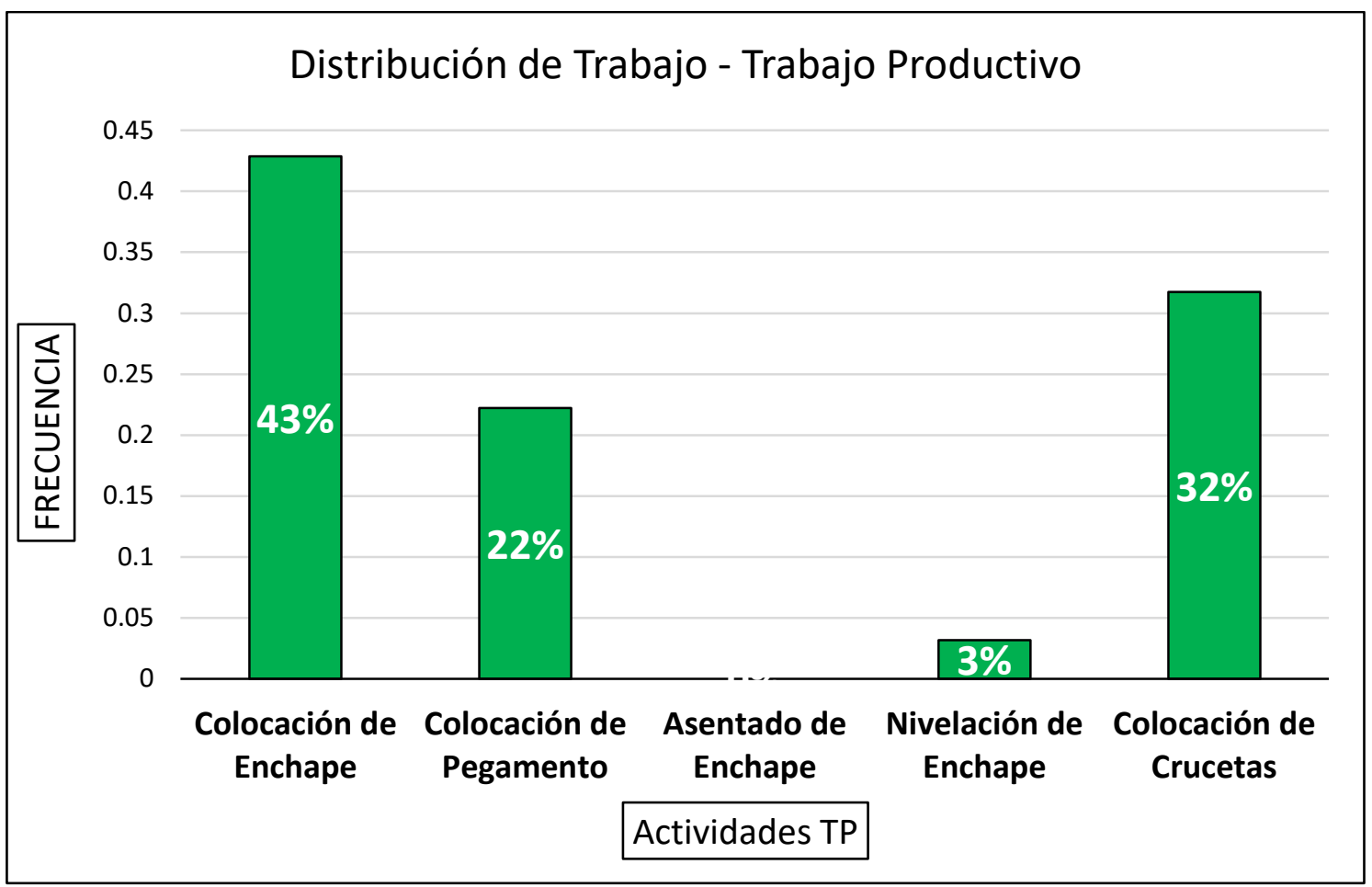

Fuente: Elaboración Propia. 
Figura $N^{\circ} 109$ : Distribución de Trabajo Contributorio - Carta Balance ${ }^{\circ} 15$

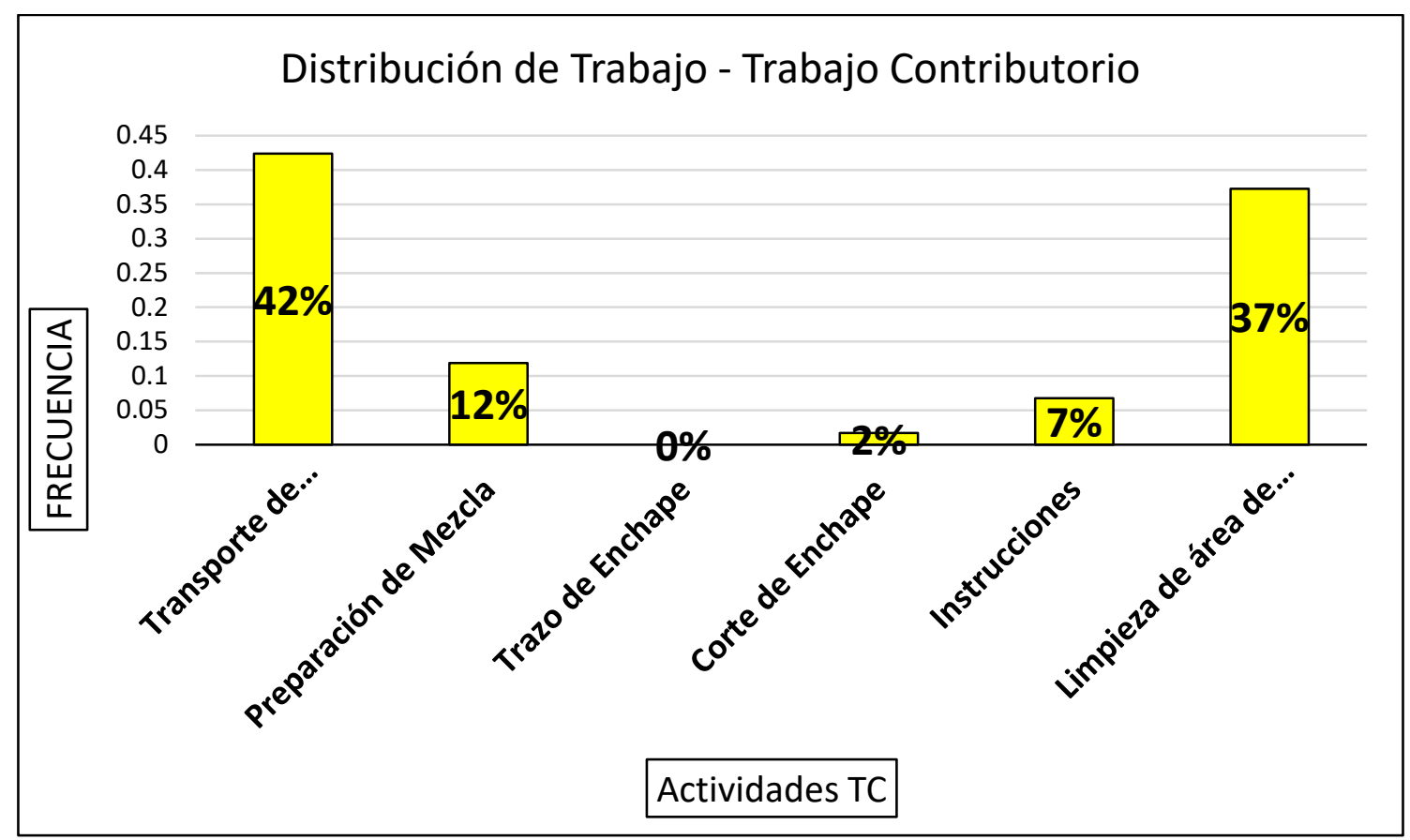

Fuente: Elaboración Propia.

Figura $N^{\circ} 110$ : Distribución de Trabajo No Contributorio - Carta Balance ${ }^{\circ} 15$

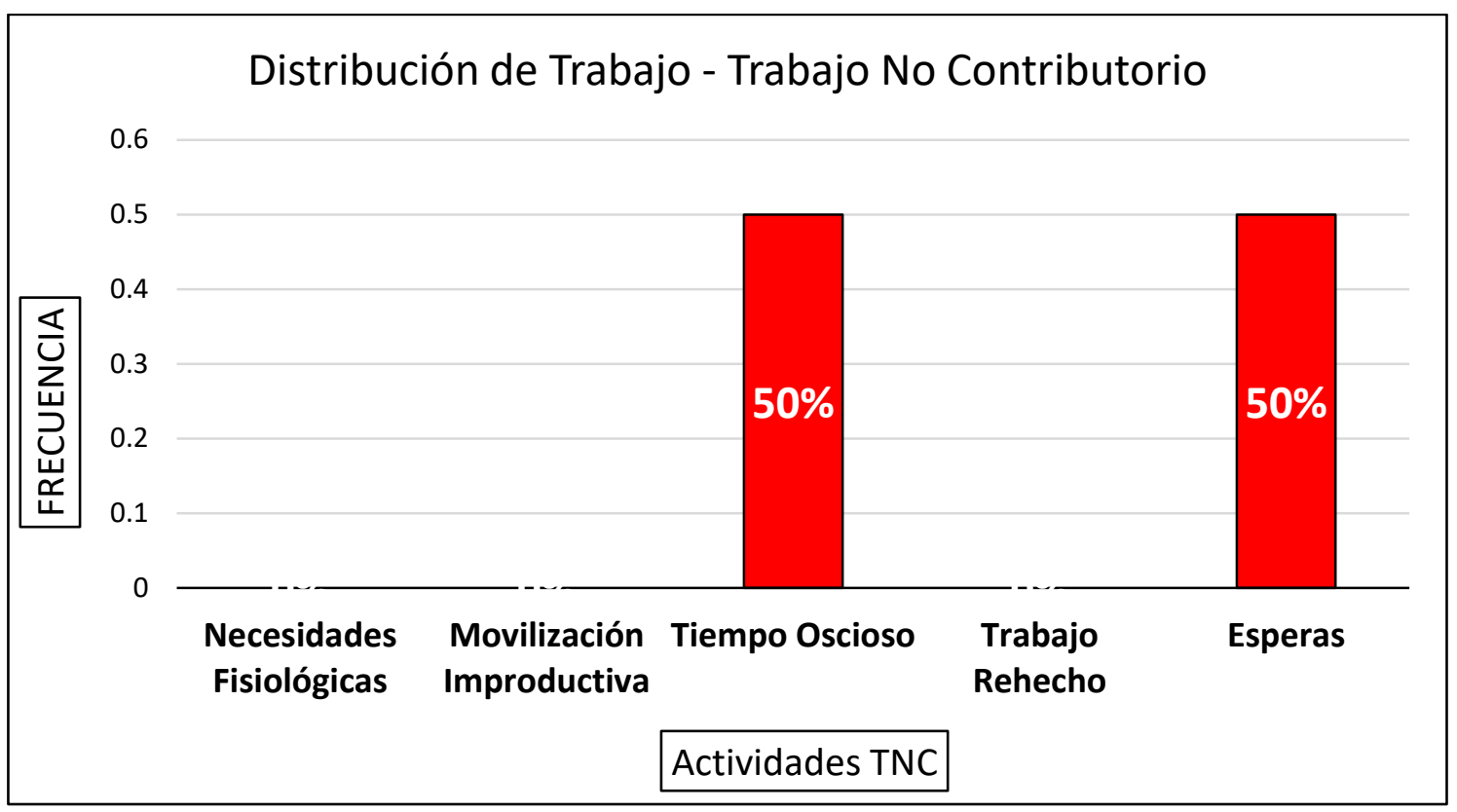

Fuente: Elaboración Propia. 
Tabla N 31: Carta Balance ${ }^{\circ} 16$ - Enchape de Cerámico

\begin{tabular}{|c|c|c|c|c|c|c|c|c|}
\hline $\mathrm{N}^{*}$ & Obrero \#1 & Obrero \#2 & 31 & $\mathrm{TM}$ & $\mathrm{TM}$ & 61 & $\mathrm{CE}$ & TM \\
\hline 1 & TM & $E$ & 32 & $\mathrm{TM}$ & $\mathrm{TM}$ & 62 & CE & TM \\
\hline 2 & BM & E & 33 & $\mathrm{TM}$ & $\mathrm{TM}$ & 63 & $C P$ & $\mathrm{TM}$ \\
\hline 3 & BM & E & 34 & L & $\mathrm{TM}$ & 64 & $\mathrm{CP}$ & TM \\
\hline 4 & BM & $E$ & 35 & l & $\mathrm{TM}$ & 65 & $\mathrm{CE}$ & TM \\
\hline 5 & $C P$ & $E$ & 36 & $L$ & $\mathrm{TM}$ & 66 & $C C$ & TM \\
\hline 6 & $C P$ & E & 37 & L & $\mathrm{TM}$ & 67 & $C C$ & TM \\
\hline 7 & NE & $E$ & 38 & l & $\mathrm{TM}$ & 68 & $C C$ & TM \\
\hline 8 & CE & $E$ & 39 & L & $\mathrm{TM}$ & 69 & $C C$ & $\mathrm{TM}$ \\
\hline 9 & CE & $E$ & 40 & L & $\mathrm{TM}$ & 70 & $C C$ & $\mathrm{TM}$ \\
\hline 10 & CE & $E$ & 41 & $C P$ & $\mathrm{TM}$ & 71 & $\mathrm{BM}$ & TM \\
\hline 11 & CE & $\mathrm{TM}$ & 42 & $C P$ & $\mathrm{TM}$ & 72 & $B M$ & $\mathrm{TM}$ \\
\hline 12 & CE & $\mathrm{TM}$ & 43 & NE & $\mathrm{TM}$ & 73 & $C P$ & TM \\
\hline 13 & CE & TM & 44 & $\mathrm{CE}$ & $\mathrm{TM}$ & 74 & NE & TM \\
\hline 14 & $\mathrm{CC}$ & $\mathrm{TM}$ & 45 & $C E$ & $\mathrm{TM}$ & 75 & NE & TM \\
\hline 15 & $C C$ & TM & 46 & NE & $\mathrm{TM}$ & 76 & NE & TM \\
\hline 16 & $\mathrm{CC}$ & $\mathrm{TM}$ & 47 & CE & $\mathrm{TM}$ & 77 & $\mathrm{CE}$ & TM \\
\hline 17 & L & TM & 48 & $C E$ & $\mathrm{TM}$ & 78 & $C E$ & TM \\
\hline 18 & L & $\mathrm{TM}$ & 49 & NE & $\mathrm{TM}$ & 79 & $C E$ & TM \\
\hline 19 & NE & $\mathrm{TM}$ & so & CE & $\mathrm{TM}$ & 80 & AP & $\mathrm{TM}$ \\
\hline 20 & $\mathrm{TM}$ & $E$ & 51 & NE & $\mathrm{TM}$ & 81 & L & $\mathrm{TM}$ \\
\hline 21 & L & E & 52 & $C P$ & $\mathrm{TM}$ & 82 & $\mathrm{TM}$ & $\mathrm{TM}$ \\
\hline 22 & 1 & $E$ & 53 & NE & $\mathrm{TM}$ & 83 & $\mathrm{l}$ & $\mathrm{TM}$ \\
\hline 23 & L & $\mathrm{TM}$ & 54 & $C P$ & $\mathrm{TM}$ & 84 & L & $\mathrm{TM}$ \\
\hline 24 & $\mathrm{TM}$ & $\mathrm{TM}$ & 55 & NE & $\mathrm{TM}$ & 85 & L & $\mathrm{TM}$ \\
\hline 25 & TM & $\mathrm{TM}$ & 56 & CE & $\mathrm{TM}$ & 86 & NE & $\mathrm{TM}$ \\
\hline 26 & $\mathrm{TM}$ & $\mathrm{TM}$ & 57 & NE & $\mathrm{TM}$ & 87 & $\mathrm{TM}$ & L \\
\hline 27 & TM & $\mathrm{TM}$ & 58 & NE & $\mathrm{TM}$ & 88 & TM & L \\
\hline 28 & TM & $\mathrm{TM}$ & 59 & NE & $\mathrm{TM}$ & 89 & $\mathrm{TM}$ & ᄂ \\
\hline 29 & $L$ & $E$ & 60 & $\mathrm{NE}$ & $\mathrm{TM}$ & 90 & COE & $L$ \\
\hline 30 & L & L & & & & & & \\
\hline
\end{tabular}

Fuente: Elaboración Propia. 
Figura Nº 111: Distribución de Trabajo de Enchape de Cerámico - Carta Balance ${ }^{\circ} 16$

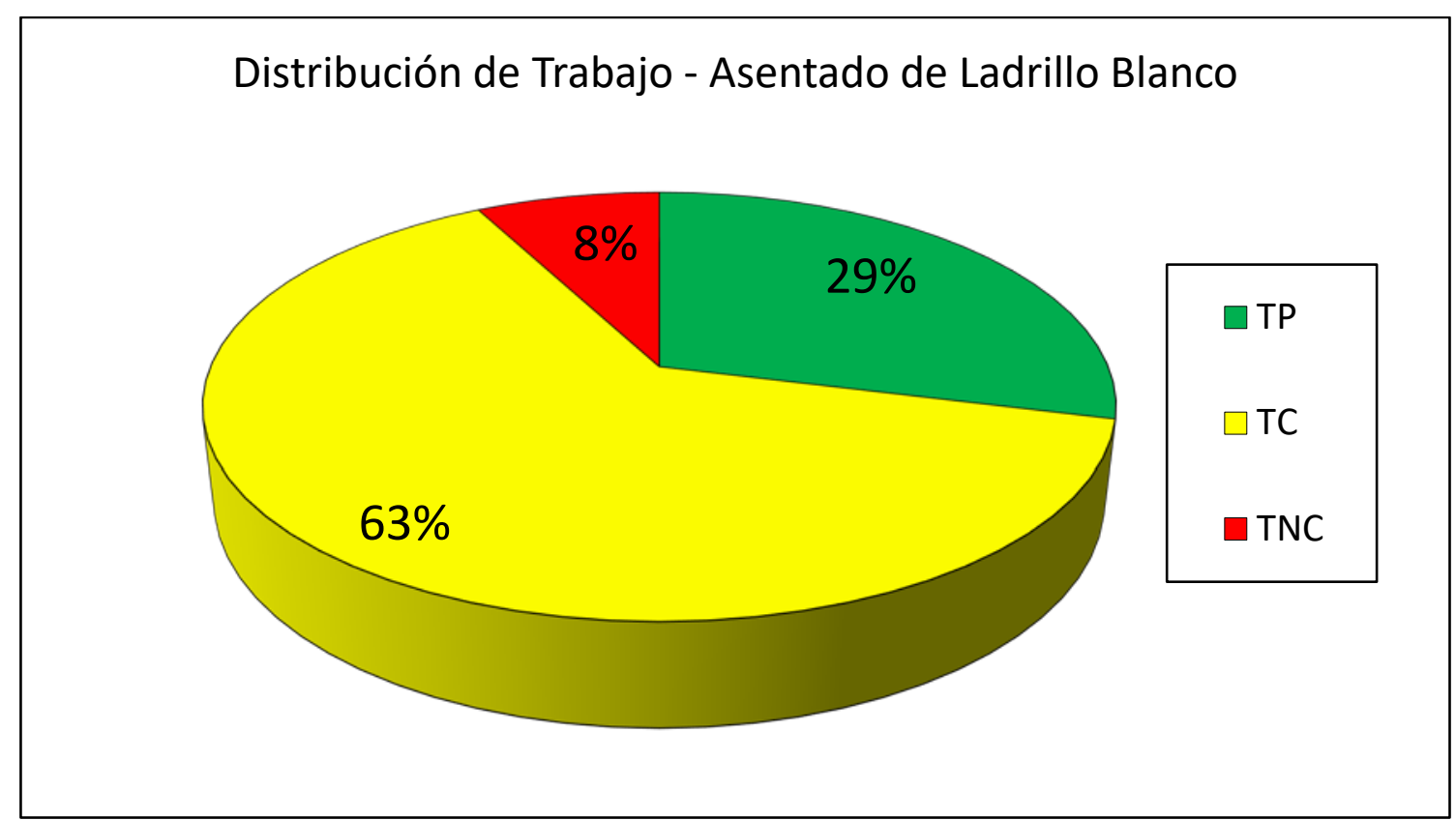

Fuente: Elaboración Propia.

Figura $N^{\circ} 112$ : Distribución de Trabajo Productivo - Carta Balance ${ }^{\circ} 16$

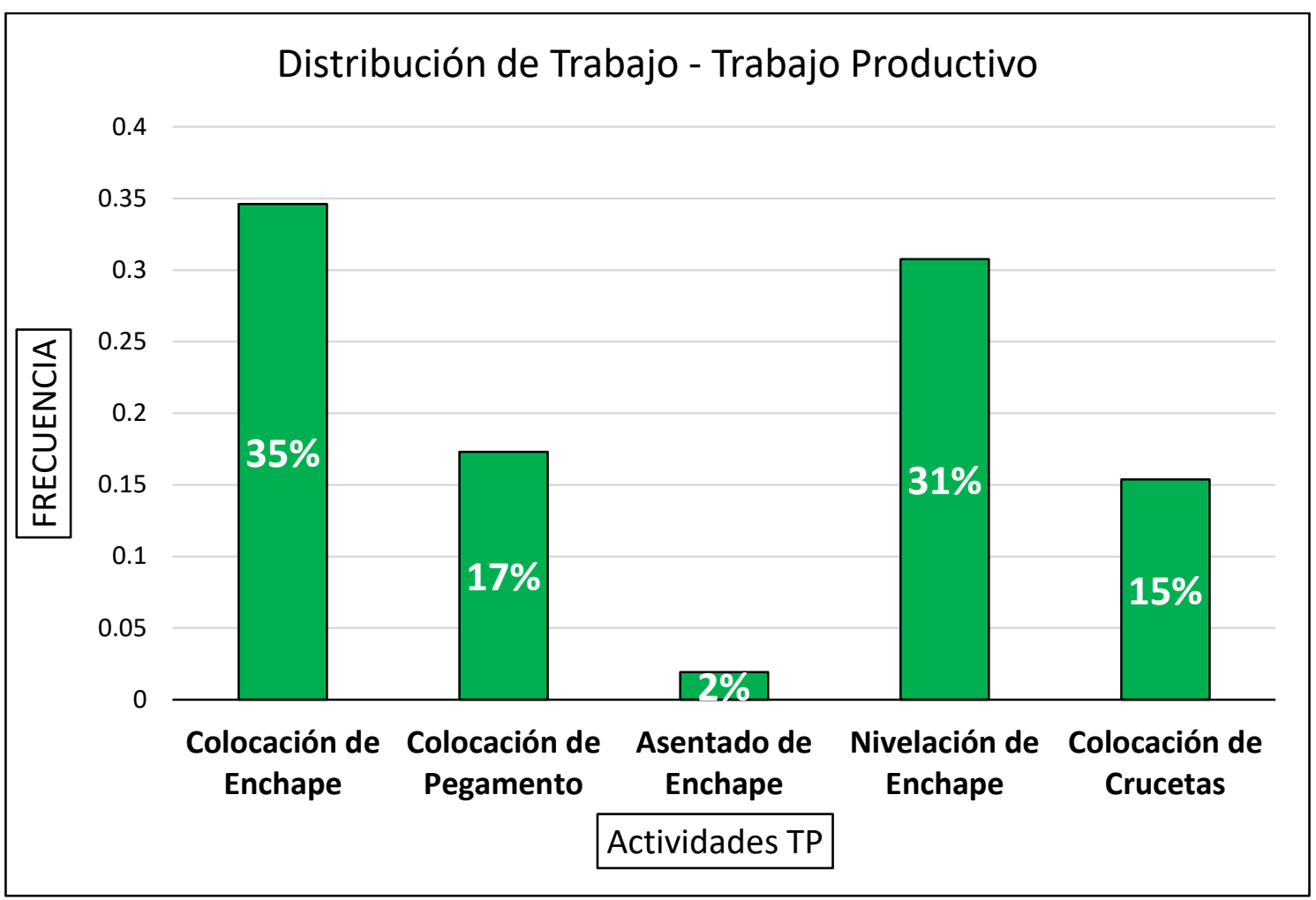

Fuente: Elaboración Propia. 
Figura $N^{\circ}$ 113: Distribución de Trabajo Contributorio - Carta Balance ${ }^{\circ} 16$

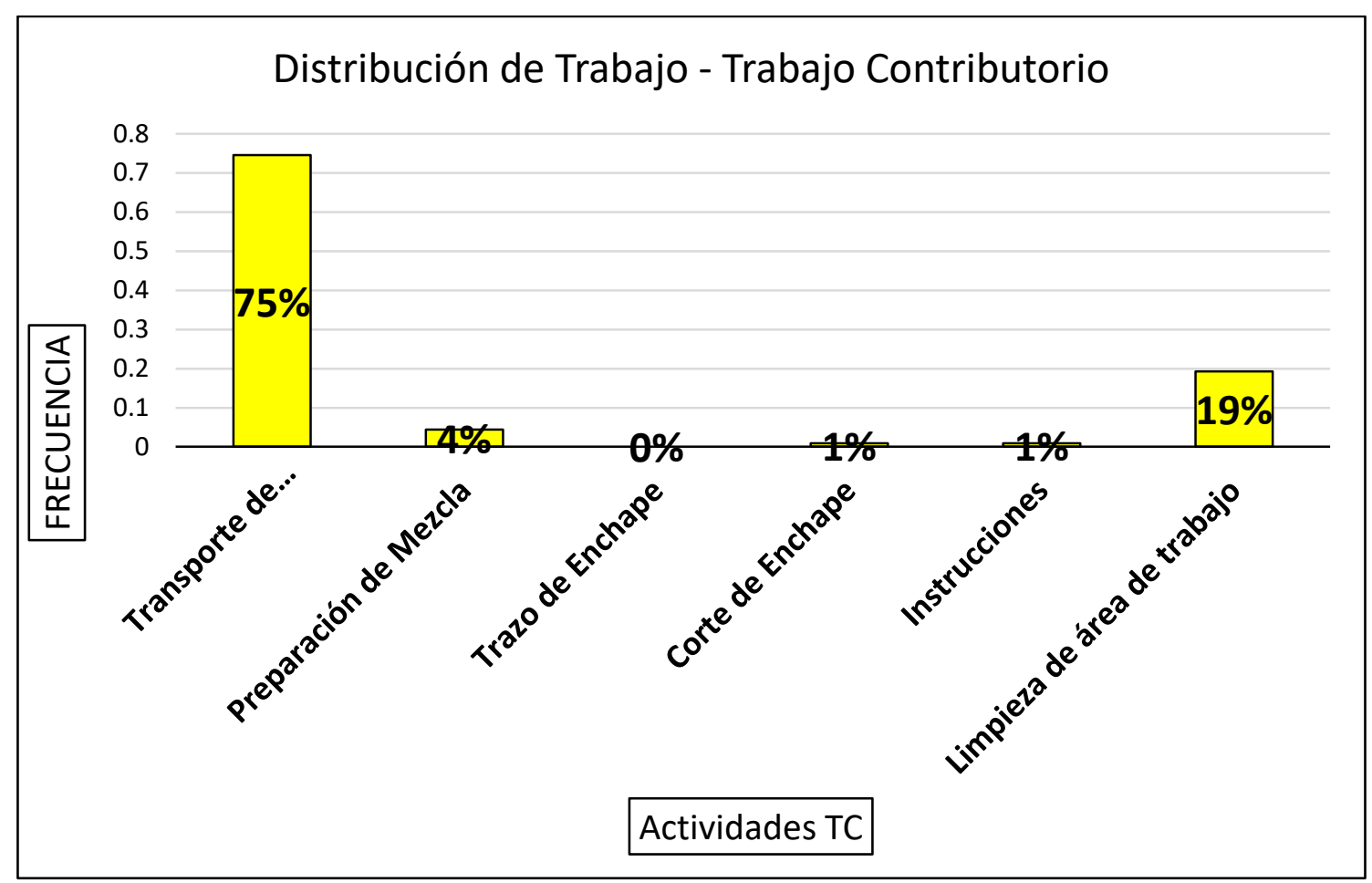

Fuente: Elaboración Propia.

Figura N ${ }^{\circ} 114$ : Distribución de Trabajo No Contributorio - Carta Balance ${ }^{\circledR} 16$

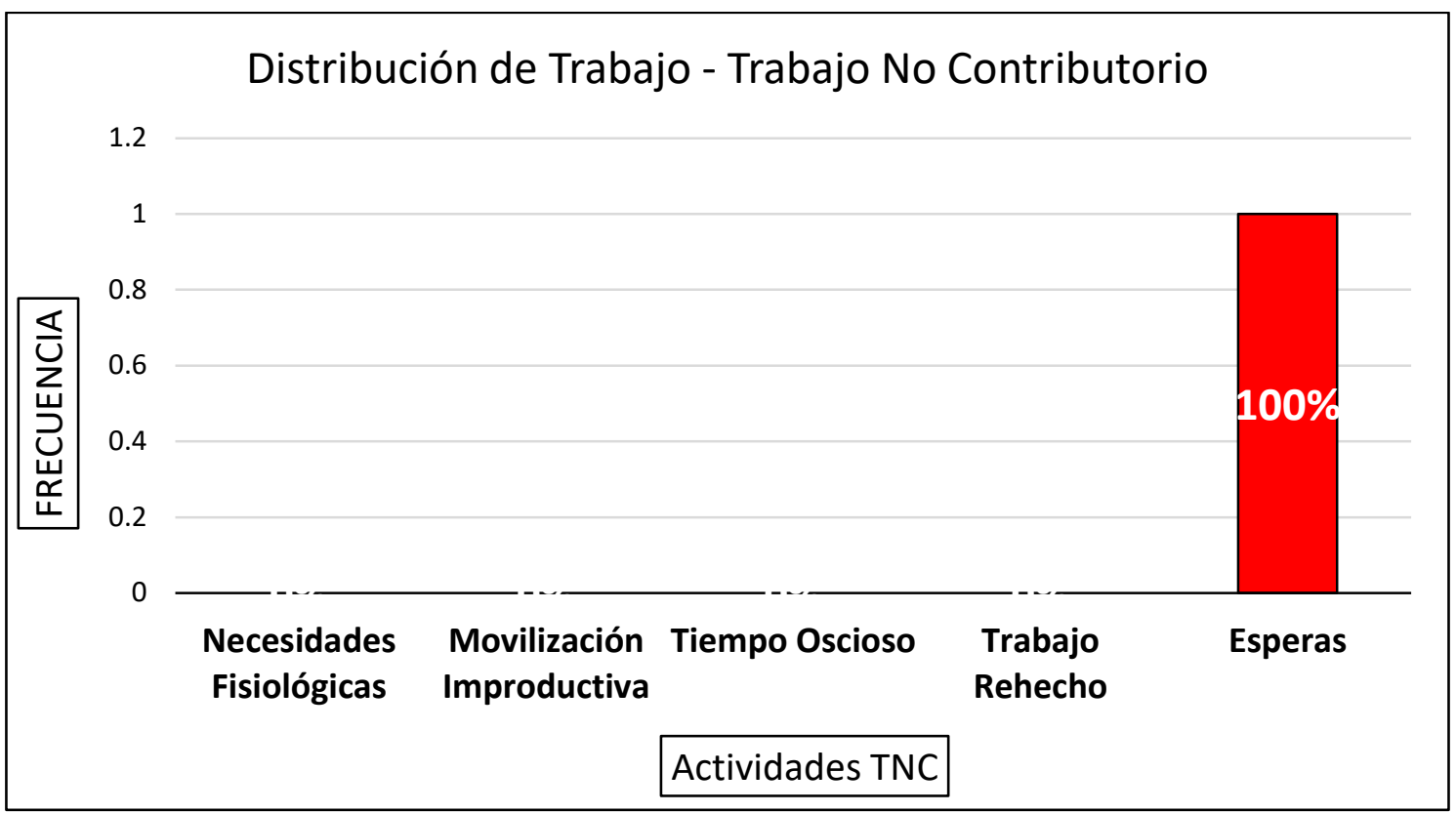

Fuente: Elaboración Propia. 
Tabla N 32: Carta Balance ${ }^{\circ} 17$ - Enchape de Cerámico

\begin{tabular}{|c|c|c|}
\hline $\mathbf{N}^{*}$ & Obrero \#1 & Obrero \#2 \\
\hline 1 & $\mathrm{CP}$ & $\mathrm{E}$ \\
\hline 2 & $\mathrm{CP}$ & $\mathrm{E}$ \\
\hline 3 & $\mathrm{CE}$ & $\mathrm{E}$ \\
\hline 4 & $\mathrm{CE}$ & $\mathrm{E}$ \\
\hline 5 & $\mathrm{CC}$ & $\mathrm{E}$ \\
\hline 6 & $\mathrm{CE}$ & $\mathrm{E}$ \\
\hline 7 & $\mathrm{CP}$ & $\mathrm{E}$ \\
\hline 8 & $\mathrm{NE}$ & $\mathrm{E}$ \\
\hline 9 & $\mathrm{CP}$ & $\mathrm{E}$ \\
\hline 10 & $\mathrm{CE}$ & $\mathrm{E}$ \\
\hline 11 & $\mathrm{CP}$ & $\mathrm{E}$ \\
\hline 12 & $\mathrm{CC}$ & $\mathrm{E}$ \\
\hline 13 & $\mathrm{CC}$ & $\mathrm{E}$ \\
\hline 14 & $\mathrm{CE}$ & $\mathrm{E}$ \\
\hline 15 & $\mathrm{CE}$ & $\mathrm{E}$ \\
\hline 16 & $\mathrm{CC}$ & $\mathrm{L}$ \\
\hline 17 & $\mathrm{COE}$ & $\mathrm{L}$ \\
\hline 18 & $\mathrm{NE}$ & $\mathrm{L}$ \\
\hline 19 & $\mathrm{CP}$ & $\mathrm{E}$ \\
\hline 20 & $\mathrm{AP}$ & $\mathrm{E}$ \\
\hline 21 & $\mathrm{CC}$ & $\mathrm{E}$ \\
\hline 22 & $\mathrm{TE}$ & $\mathrm{E}$ \\
\hline 23 & $\mathrm{COE}$ & $\mathrm{E}$ \\
\hline 24 & $\mathrm{COE}$ & $\mathrm{E}$ \\
\hline 25 & $\mathrm{COE}$ & $\mathrm{E}$ \\
\hline 26 & $\mathrm{COE}$ & $\mathrm{E}$ \\
\hline 27 & $\mathrm{CE}$ & $\mathrm{E}$ \\
\hline 28 & $\mathrm{CE}$ & $\mathrm{E}$ \\
\hline 29 & $\mathrm{CE}$ & $\mathrm{E}$ \\
\hline 30 & $\mathrm{CP}$ & $\mathrm{E}$ \\
\hline
\end{tabular}

\begin{tabular}{|c|c|c|}
\hline 31 & CE & E \\
\hline 32 & CP & E \\
\hline 33 & CP & E \\
\hline 34 & CP & E \\
\hline 35 & TE & E \\
\hline 36 & CE & E \\
\hline 37 & NE & E \\
\hline 38 & COE & E \\
\hline 39 & TE & L \\
\hline 40 & COE & L \\
\hline 41 & CP & L \\
\hline 42 & NE & L \\
\hline 43 & NE & L \\
\hline 44 & BM & L \\
\hline 45 & BM & L \\
\hline 46 & BM & E \\
\hline 47 & CP & E \\
\hline 48 & CC & E \\
\hline 49 & CE & E \\
\hline 50 & CC & E \\
\hline 51 & CE & E \\
\hline 52 & CP & E \\
\hline 53 & NE & E \\
\hline 54 & CE & E \\
\hline 55 & CC & E \\
\hline 56 & CP & E \\
\hline 57 & NE & E \\
\hline 58 & CC & E \\
\hline 59 & CP & E \\
\hline 60 & CP & E \\
\hline & & \\
\hline
\end{tabular}

\begin{tabular}{|c|c|c|}
\hline 61 & $\mathrm{CE}$ & $\mathrm{E}$ \\
\hline 62 & $\mathrm{CC}$ & $\mathrm{E}$ \\
\hline 63 & $\mathrm{CP}$ & $\mathrm{E}$ \\
\hline 64 & $\mathrm{CE}$ & $\mathrm{E}$ \\
\hline 65 & $\mathrm{CP}$ & $\mathrm{E}$ \\
\hline 66 & $\mathrm{CC}$ & $\mathrm{E}$ \\
\hline 67 & $\mathrm{CP}$ & $\mathrm{E}$ \\
\hline 68 & $\mathrm{MI}$ & $\mathrm{E}$ \\
\hline 69 & $\mathrm{CC}$ & $\mathrm{E}$ \\
\hline 70 & $\mathrm{CP}$ & $\mathrm{E}$ \\
\hline 71 & $\mathrm{CE}$ & $\mathrm{E}$ \\
\hline 72 & $\mathrm{CC}$ & $\mathrm{E}$ \\
\hline 73 & $\mathrm{CP}$ & $\mathrm{E}$ \\
\hline 74 & $\mathrm{CE}$ & $\mathrm{E}$ \\
\hline 75 & $\mathrm{CC}$ & $\mathrm{E}$ \\
\hline 76 & $\mathrm{I}$ & $\mathrm{E}$ \\
\hline 77 & $\mathrm{TM}$ & $\mathrm{E}$ \\
\hline 78 & $\mathrm{BM}$ & $\mathrm{E}$ \\
\hline 79 & $\mathrm{BM}$ & $\mathrm{E}$ \\
\hline 80 & $\mathrm{BM}$ & $\mathrm{E}$ \\
\hline 81 & $\mathrm{TM}$ & $\mathrm{E}$ \\
\hline 82 & $\mathrm{I}$ & $\mathrm{E}$ \\
\hline 83 & $\mathrm{CP}$ & $\mathrm{TM}$ \\
\hline 84 & $\mathrm{CP}$ & $\mathrm{TM}$ \\
\hline 85 & $\mathrm{CE}$ & $\mathrm{TM}$ \\
\hline 86 & $\mathrm{CP}$ & $\mathrm{TM}$ \\
\hline 87 & $\mathrm{CE}$ & $\mathrm{TM}$ \\
\hline 88 & $\mathrm{CC}$ & $\mathrm{TM}$ \\
\hline 89 & $\mathrm{CC}$ & $\mathrm{TM}$ \\
\hline 90 & $\mathrm{CC}$ & $\mathrm{TM}$ \\
\hline & & \\
\hline
\end{tabular}

Fuente: Elaboración Propia. 
Figura Nº 115: Distribución de Trabajo de Enchape de Cerámico - Carta Balance ${ }^{\circ} 17$

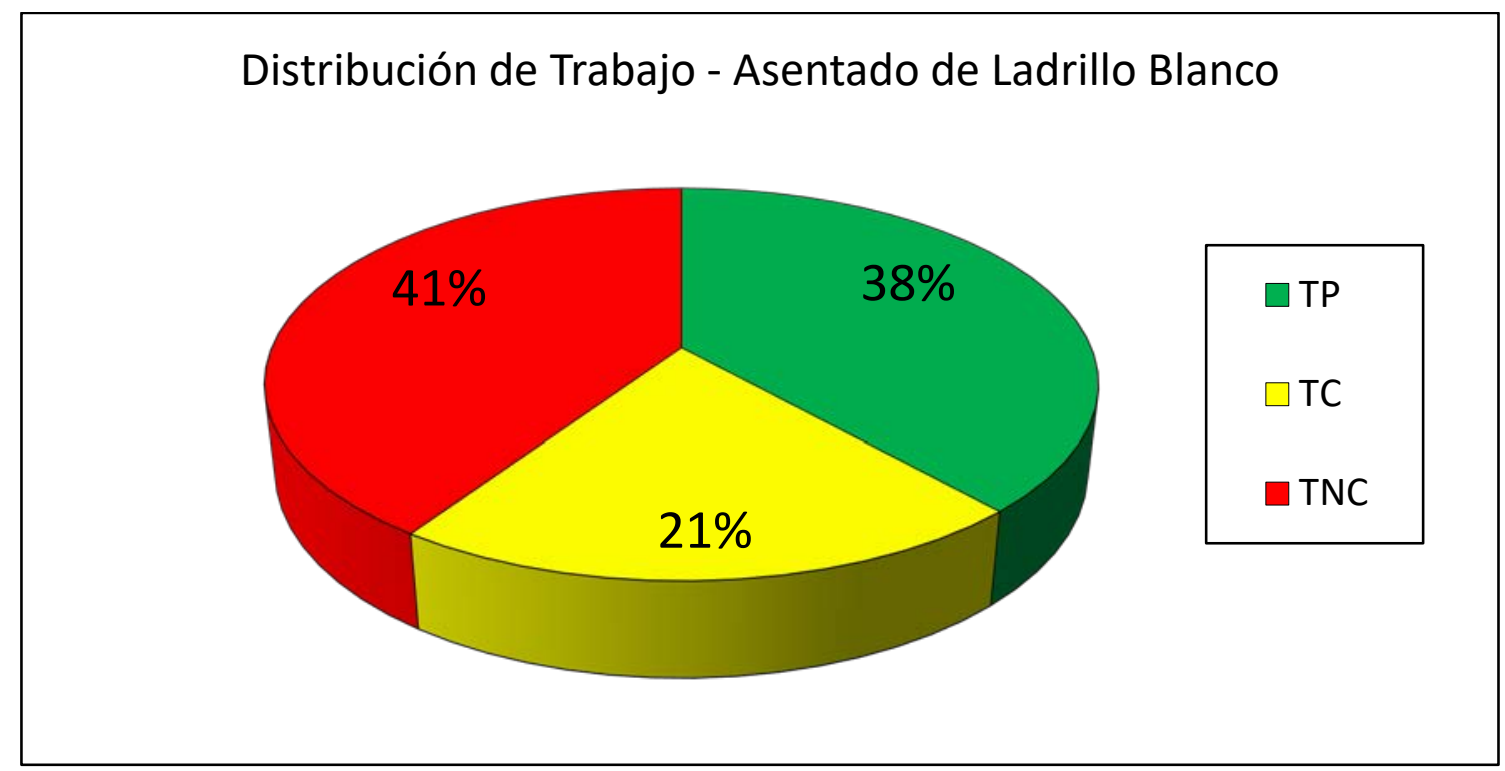

Fuente: Elaboración Propia.

Figura $N^{\circ} 116$ : Distribución de Trabajo Productivo - Carta Balance ${ }^{\circ} 17$

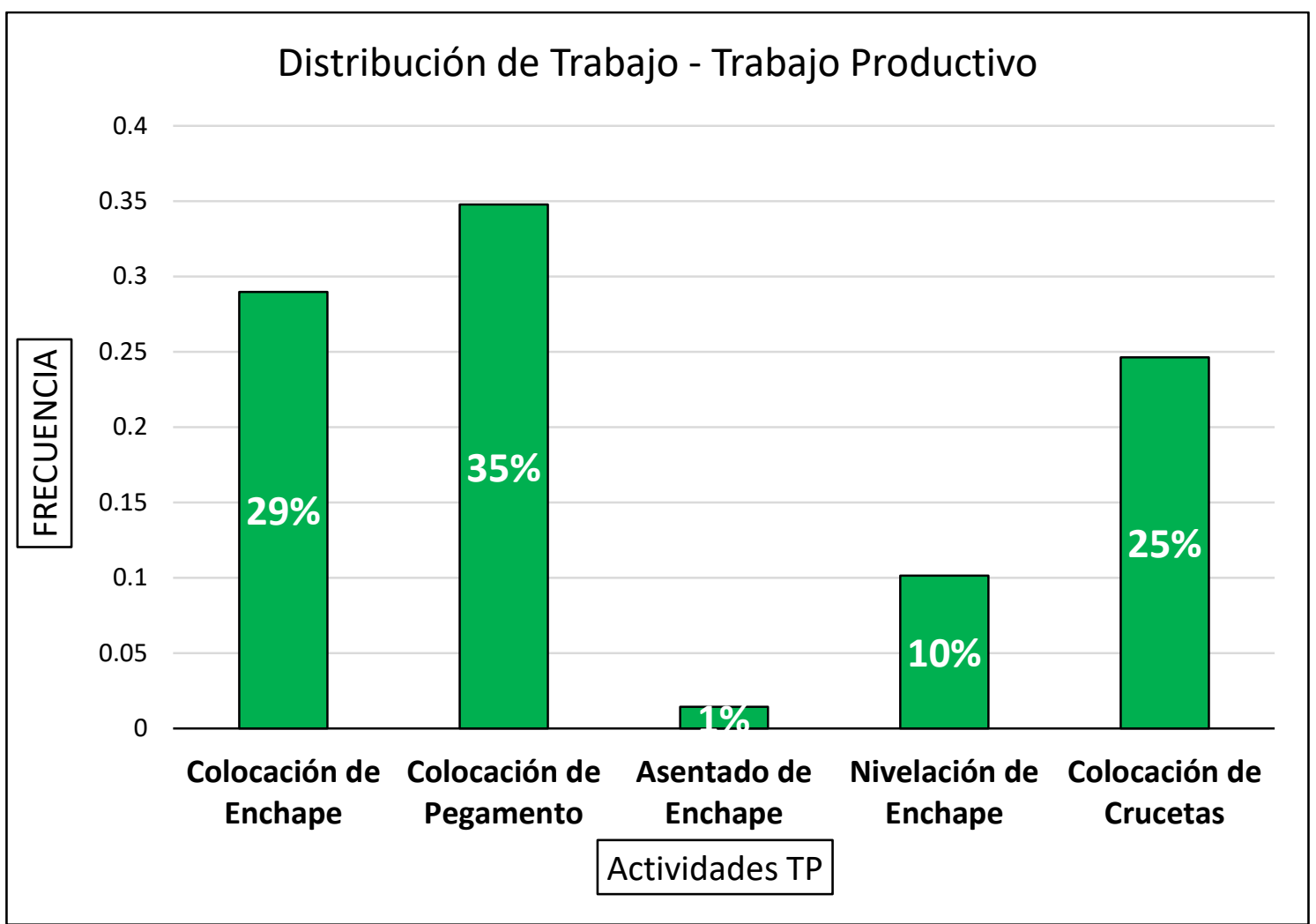

Fuente: Elaboración Propia. 
Figura $N^{\circ} 117$ : Distribución de Trabajo Contributorio - Carta Balance ${ }^{\circ} 17$

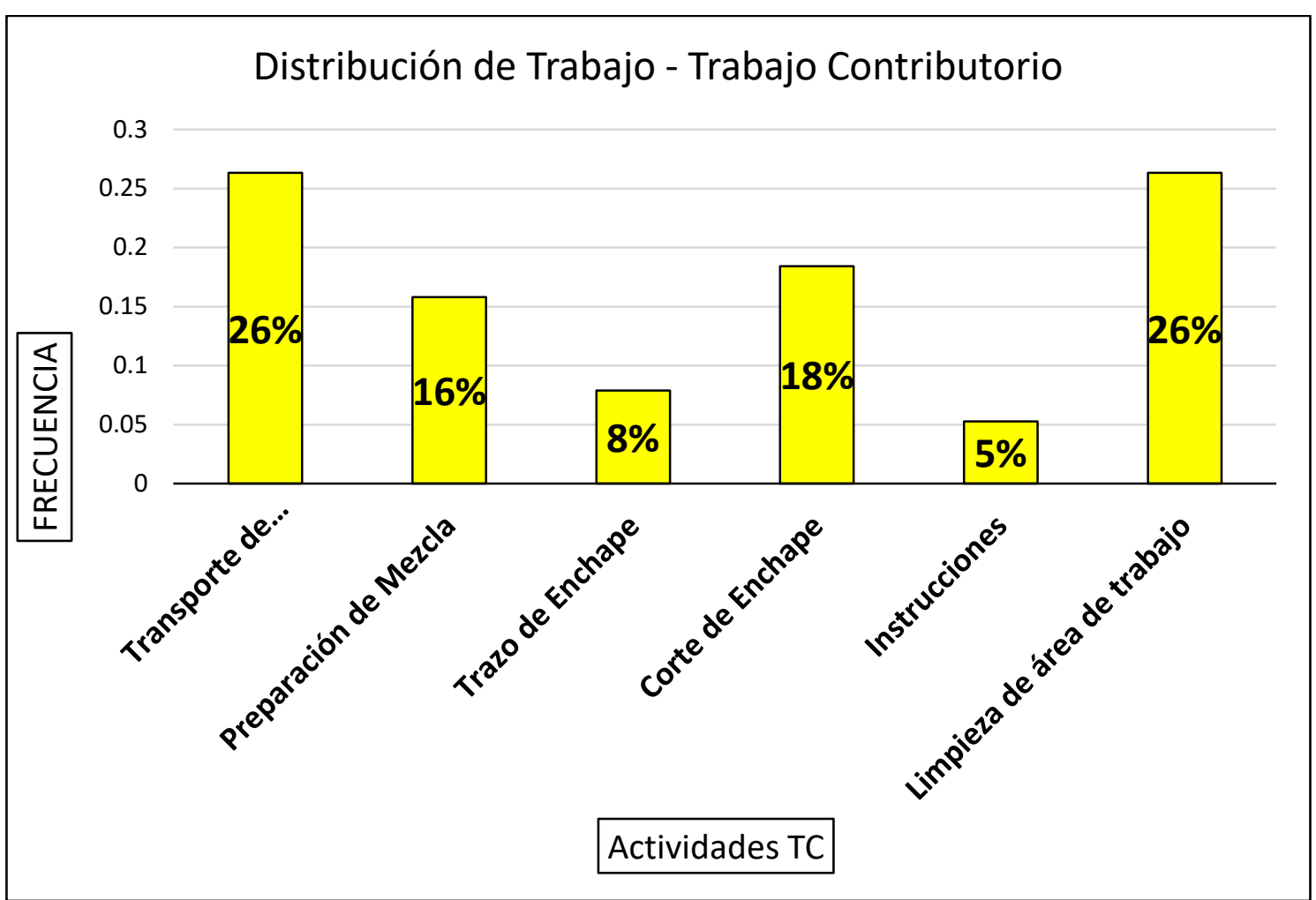

Fuente: Elaboración Propia.

Figura $N^{\circ} 118$ : Distribución de Trabajo No Contributorio - Carta Balance ${ }^{\circ} 17$

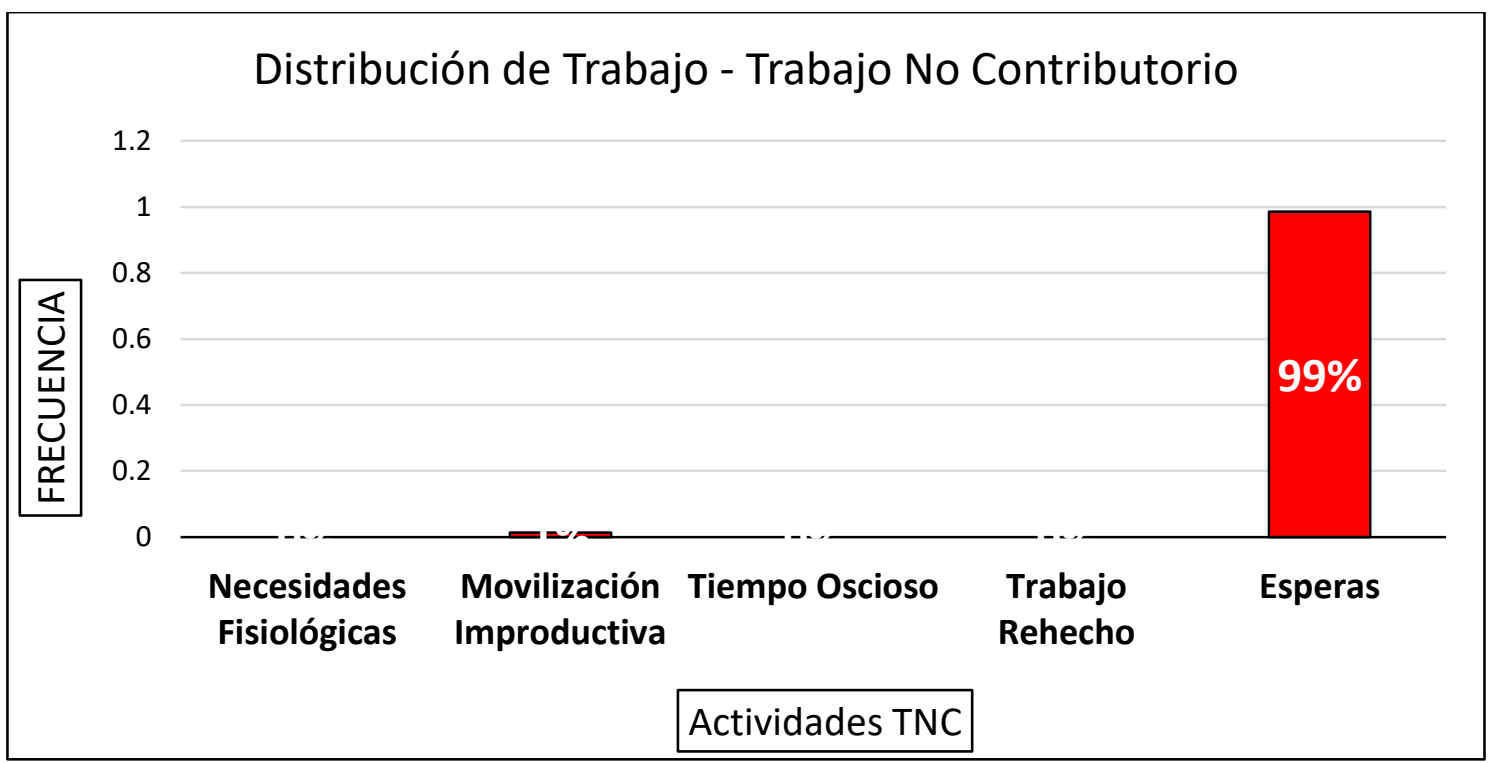

Fuente: Elaboración Propia. 
Tabla Nº 33: Carta Balance ${ }^{\circ} 18$ - Enchape de Cerámico

\begin{tabular}{|c|c|c|c|c|c|c|c|c|}
\hline $\mathrm{N}^{*}$ & Obrero \#1 & Obrero $\# 2$ & 31 & $C P$ & $E$ & 61 & CE & $E$ \\
\hline 1 & $A P$ & L & 32 & $A P$ & E & 62 & $C P$ & $\mathrm{E}$ \\
\hline 2 & AP & l & 33 & $\mathrm{CP}$ & E & 63 & CE & E \\
\hline 3 & $C C$ & TO & 34 & $\mathrm{CE}$ & $E$ & 64 & $C P$ & $E$ \\
\hline 4 & NE & TO & 35 & $C P$ & E & 65 & $\mathrm{NE}$ & $E$ \\
\hline 5 & $\mathrm{COE}$ & TO & 36 & $\mathrm{CE}$ & E & 66 & 1 & $\mathrm{E}$ \\
\hline 6 & $\mathrm{COE}$ & 1 & 37 & $\mathrm{CE}$ & $E$ & 67 & $B M$ & $E$ \\
\hline 7 & COE & TM & 38 & NE & E & 68 & $B M$ & $E$ \\
\hline 8 & COE & $\mathrm{TM}$ & 39 & TE & $E$ & 69 & $\mathrm{BM}$ & $\mathrm{E}$ \\
\hline 9 & NE & $\mathrm{TM}$ & 40 & COE & $E$ & 70 & 1 & $\mathrm{E}$ \\
\hline 10 & $C P$ & $\mathrm{TM}$ & 41 & NE & E & 71 & $\mathrm{CP}$ & $E$ \\
\hline 11 & $C E$ & $\mathrm{TM}$ & 42 & $\mathrm{CC}$ & $E$ & 72 & CE & $\mathrm{E}$ \\
\hline 12 & $A P$ & $\mathrm{TM}$ & 43 & $\mathrm{~L}$ & $E$ & 73 & CE & $\mathrm{E}$ \\
\hline 13 & TE & $E$ & 44 & $\mathrm{TM}$ & E & 74 & $\mathrm{CP}$ & $E$ \\
\hline 14 & CE & $E$ & 45 & MI & $E$ & 75 & $\mathrm{CE}$ & $\mathrm{E}$ \\
\hline 15 & NE & $E$ & 46 & MI & $E$ & 76 & CE & $\mathrm{E}$ \\
\hline 16 & $C P$ & E & 47 & $\mathrm{TM}$ & E & 77 & $\mathrm{CP}$ & $E$ \\
\hline 17 & NE & $E$ & 48 & $\mathrm{TM}$ & $\mathrm{E}$ & 78 & $\mathrm{CC}$ & $\mathrm{E}$ \\
\hline 18 & $\mathrm{CE}$ & $E$ & 49 & $t$ & $E$ & 79 & CE & $E$ \\
\hline 19 & $C E$ & $E$ & 50 & $\mathrm{~L}$ & E & 80 & $\mathrm{CP}$ & $\mathrm{E}$ \\
\hline 20 & CE & $E$ & 51 & $\mathrm{~L}$ & E & 81 & CE & $\mathrm{E}$ \\
\hline 21 & NE & $E$ & 52 & TE & $E$ & 82 & $\mathrm{CC}$ & $\mathrm{E}$ \\
\hline 22 & $C P$ & $E$ & 53 & TE & $E$ & 83 & $\mathrm{NE}$ & $\mathrm{E}$ \\
\hline 23 & $\mathrm{CE}$ & E & 54 & COE & E & 84 & $\mathrm{CP}$ & E \\
\hline 24 & $C C$ & $E$ & 55 & COE & $E$ & 85 & CE & $\mathrm{E}$ \\
\hline 25 & $\mathrm{CE}$ & $E$ & 56 & $\operatorname{COE}$ & $E$ & 86 & $\mathrm{CC}$ & $\mathrm{E}$ \\
\hline 26 & $C P$ & $E$ & 57 & $C P$ & $E$ & 87 & $C P$ & $\mathrm{E}$ \\
\hline 27 & $\mathrm{CE}$ & $E$ & 58 & $C P$ & $E$ & 88 & TO & TO \\
\hline 28 & $C C$ & $E$ & 59 & $\mathrm{CC}$ & $E$ & 89 & $C P$ & $\mathrm{E}$ \\
\hline 29 & $C P$ & $E$ & 60 & $\mathrm{CE}$ & $E$ & 90 & $\mathrm{CC}$ & $E$ \\
\hline 30 & TE & $E$ & & & & & & \\
\hline
\end{tabular}

Fuente: Elaboración Propia. 
Figura Nº 119: Distribución de Trabajo de Enchape de Cerámico - Carta Balance ${ }^{\circ} 18$

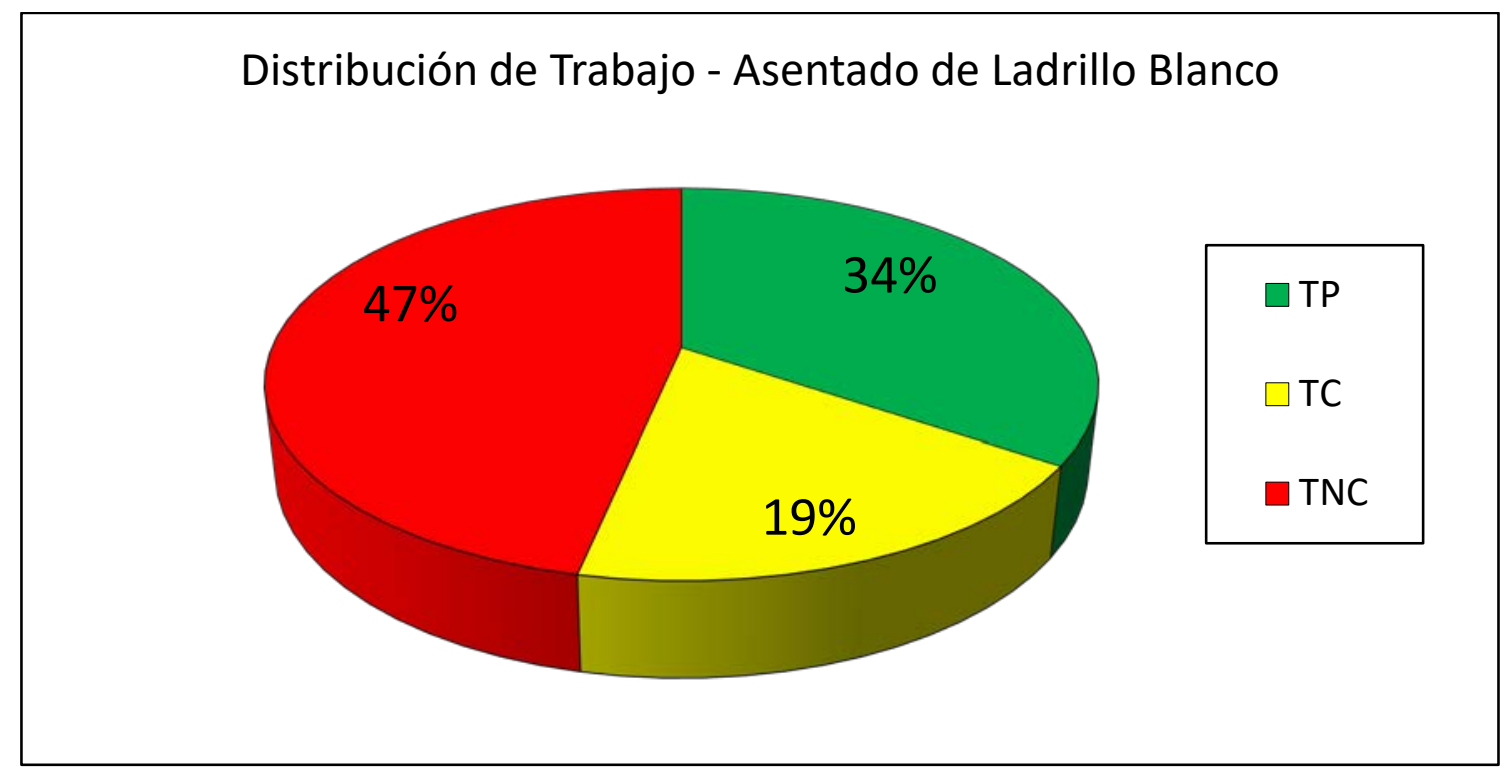

Fuente: Elaboración Propia.

Figura $N^{\circ} 120$ : Distribución de Trabajo Productivo - Carta Balance ${ }^{\circ} 18$

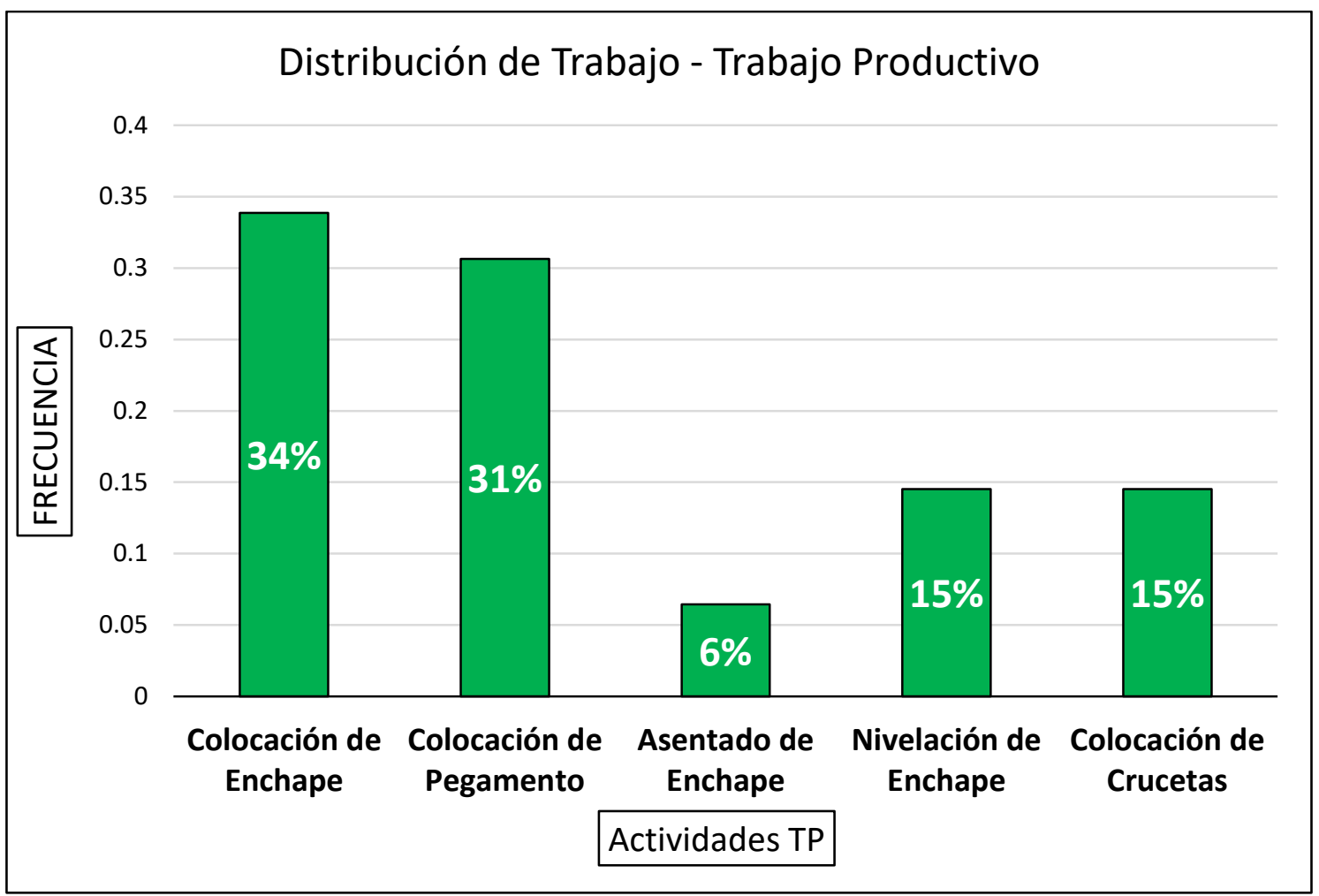

Fuente: Elaboración Propia. 
Figura $N^{\circ} 121$ : Distribución de Trabajo Contributorio - Carta Balance ${ }^{\circ} 18$

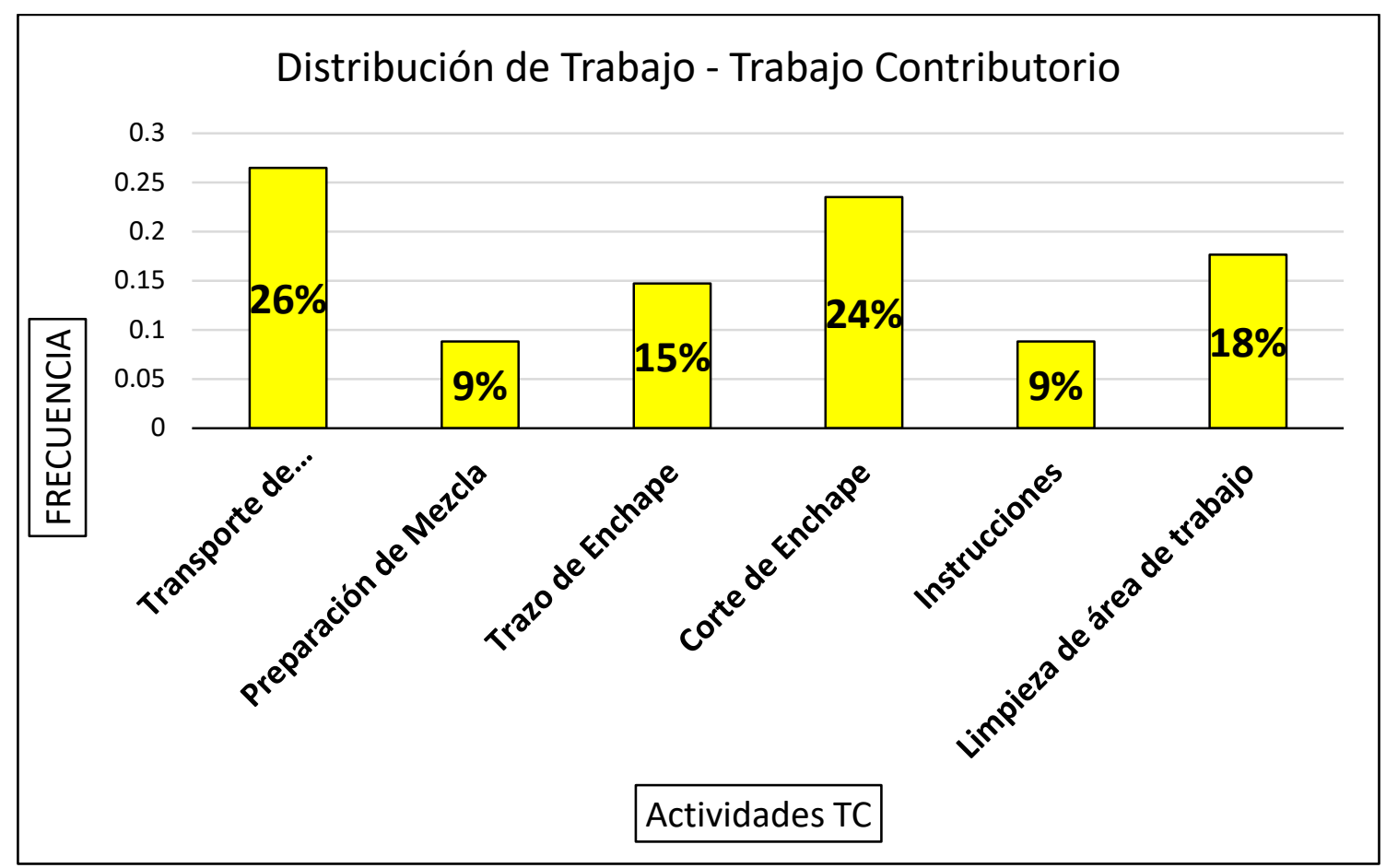

Fuente: Elaboración Propia.

Figura N 122: Distribución de Trabajo No Contributorio - Carta Balance ${ }^{\circledR} 18$

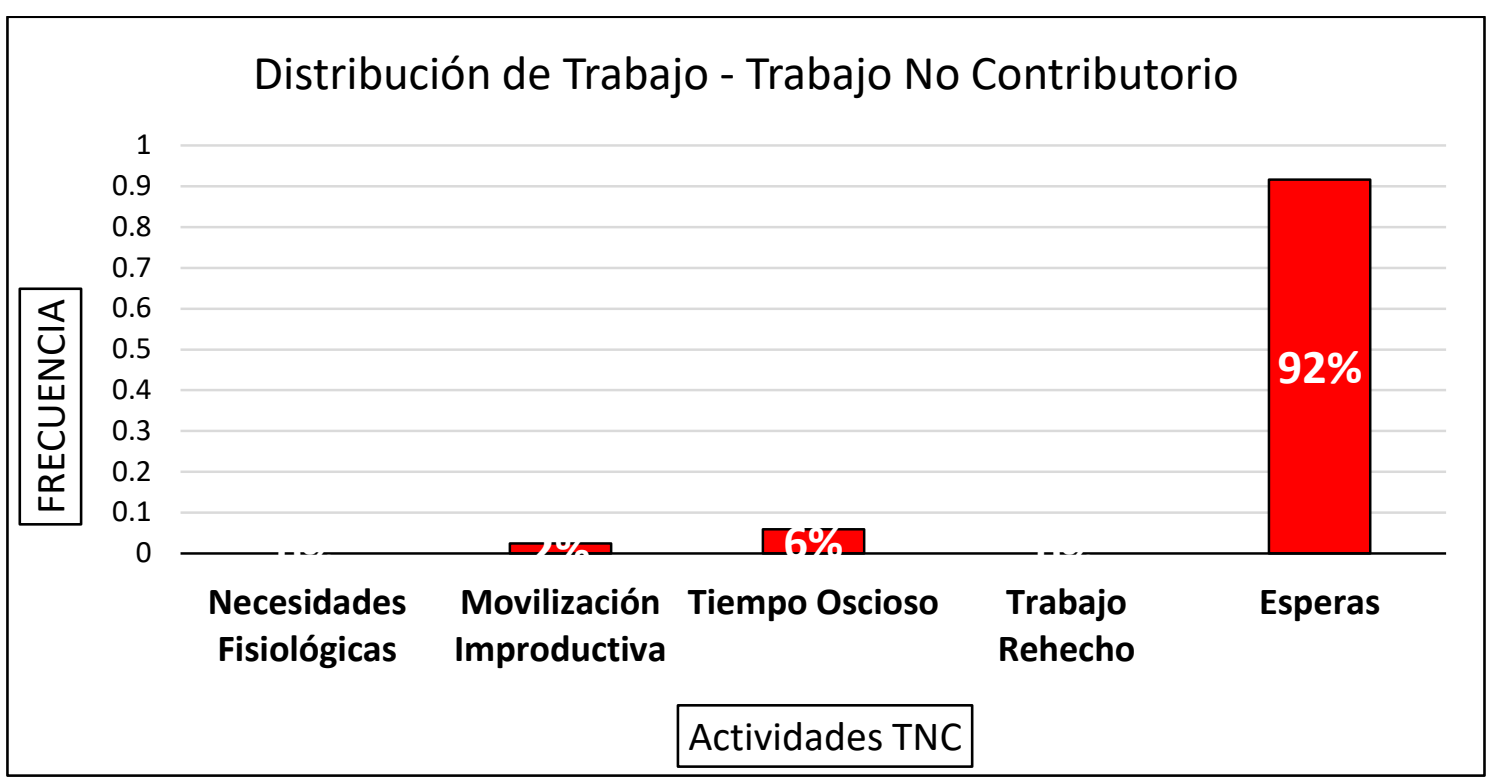

Fuente: Elaboración Propia. 
Tabla N 34: Carta Balance ${ }^{\circ} 19$ - Enchape de Cerámico

\begin{tabular}{|c|c|c|}
\hline $\mathbf{N}^{*}$ & Obrero $\# 1$ & Obrero $\# 2$ \\
\hline 1 & TE & $\mathrm{TM}$ \\
\hline 2 & TE & $\mathrm{TM}$ \\
\hline 3 & TE & $\mathrm{TM}$ \\
\hline 4 & TE & $\mathrm{TM}$ \\
\hline 5 & TE & $\mathrm{TM}$ \\
\hline 6 & L & $\mathrm{MI}$ \\
\hline 7 & L & $E$ \\
\hline 8 & TE & 1 \\
\hline 9 & TE & TE \\
\hline 10 & $\mathrm{TM}$ & $\mathrm{TM}$ \\
\hline 11 & $\mathrm{TM}$ & $\mathrm{TM}$ \\
\hline 12 & L & E \\
\hline 13 & L & E \\
\hline 14 & L & $E$ \\
\hline 15 & L & E \\
\hline 16 & $\mathrm{TM}$ & $E$ \\
\hline 17 & $\mathrm{TM}$ & TO \\
\hline 18 & $\mathrm{TM}$ & TO \\
\hline 19 & L & $E$ \\
\hline 20 & $\mathrm{TM}$ & TO \\
\hline 21 & $\mathrm{BM}$ & $\mathrm{TM}$ \\
\hline 22 & BM & E \\
\hline 23 & $\mathrm{TM}$ & $\mathrm{TM}$ \\
\hline 24 & TE & $E$ \\
\hline 25 & COE & $E$ \\
\hline 26 & COE & E \\
\hline 27 & L & $E$ \\
\hline 28 & L & E \\
\hline 29 & COE & $E$ \\
\hline 30 & COE & E \\
\hline
\end{tabular}

\begin{tabular}{|c|c|c|}
\hline 31 & TM & E \\
\hline 32 & CP & E \\
\hline 33 & CP & E \\
\hline 34 & CE & E \\
\hline 35 & AP & E \\
\hline 36 & CP & TO \\
\hline 37 & CE & TO \\
\hline 38 & CE & TO \\
\hline 39 & CC & TO \\
\hline 40 & CE & TO \\
\hline 41 & CP & TO \\
\hline 42 & AP & TO \\
\hline 43 & CP & TO \\
\hline 44 & CC & TO \\
\hline 45 & $\mathrm{CC}$ & TO \\
\hline 46 & CP & TO \\
\hline 47 & NE & E \\
\hline 48 & CP & TM \\
\hline 49 & CC & TM \\
\hline 50 & CE & TM \\
\hline 51 & TM & TM \\
\hline 52 & NE & TM \\
\hline 53 & BM & TM \\
\hline 54 & BM & TM \\
\hline 55 & BM & TM \\
\hline 56 & BM & TM \\
\hline 57 & BM & TM \\
\hline 58 & NE & TM \\
\hline 59 & NE & TM \\
\hline 60 & CP & TM \\
\hline & & \\
\hline
\end{tabular}

\begin{tabular}{|c|c|c|}
\hline 61 & CC & TM \\
\hline 62 & AP & TM \\
\hline 63 & CP & TM \\
\hline 64 & CC & TM \\
\hline 65 & NE & TM \\
\hline 66 & CC & TM \\
\hline 67 & TM & TM \\
\hline 68 & TM & TM \\
\hline 69 & TM & TM \\
\hline 70 & TM & TM \\
\hline 71 & COE & TM \\
\hline 72 & NE & TM \\
\hline 73 & CP & TM \\
\hline 74 & CE & TM \\
\hline 75 & CC & TM \\
\hline 76 & CE & TM \\
\hline 77 & CP & TM \\
\hline 78 & CE & TM \\
\hline 79 & AP & TM \\
\hline 80 & TO & TM \\
\hline 81 & TO & TM \\
\hline 82 & TO & TM \\
\hline 83 & CP & TM \\
\hline 84 & CE & TM \\
\hline 85 & CP & TM \\
\hline 86 & CE & TM \\
\hline 87 & $C C$ & TM \\
\hline 88 & TM & TM \\
\hline 89 & BM & TM \\
\hline 90 & BM & TM \\
\hline
\end{tabular}

Fuente: Elaboración Propia. 
Figura Nº 123: Distribución de Trabajo de Enchape de Cerámico - Carta Balance 19

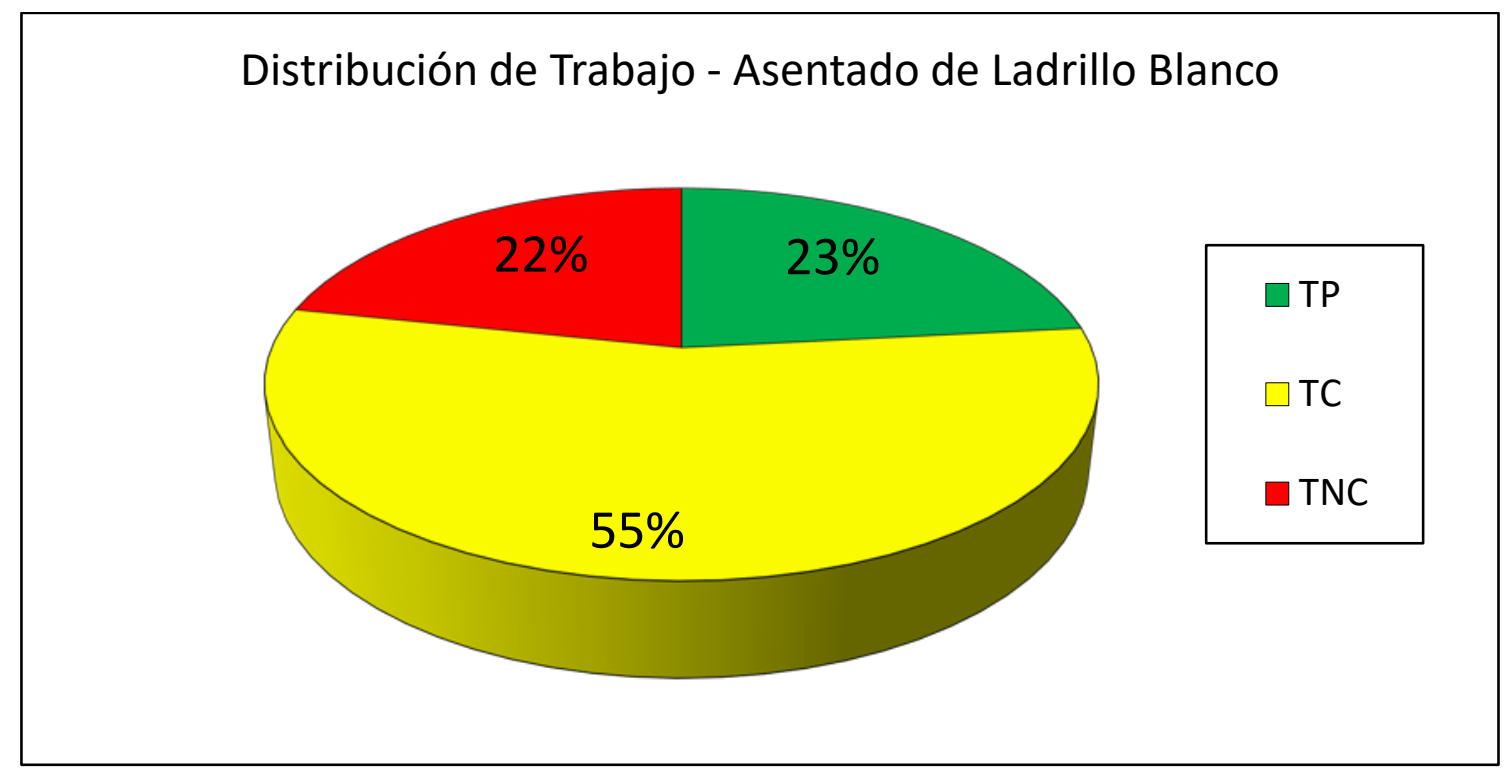

Fuente: Elaboración Propia.

Figura No 124: Distribución de Trabajo Productivo - Carta Balance ${ }^{\circ} 19$

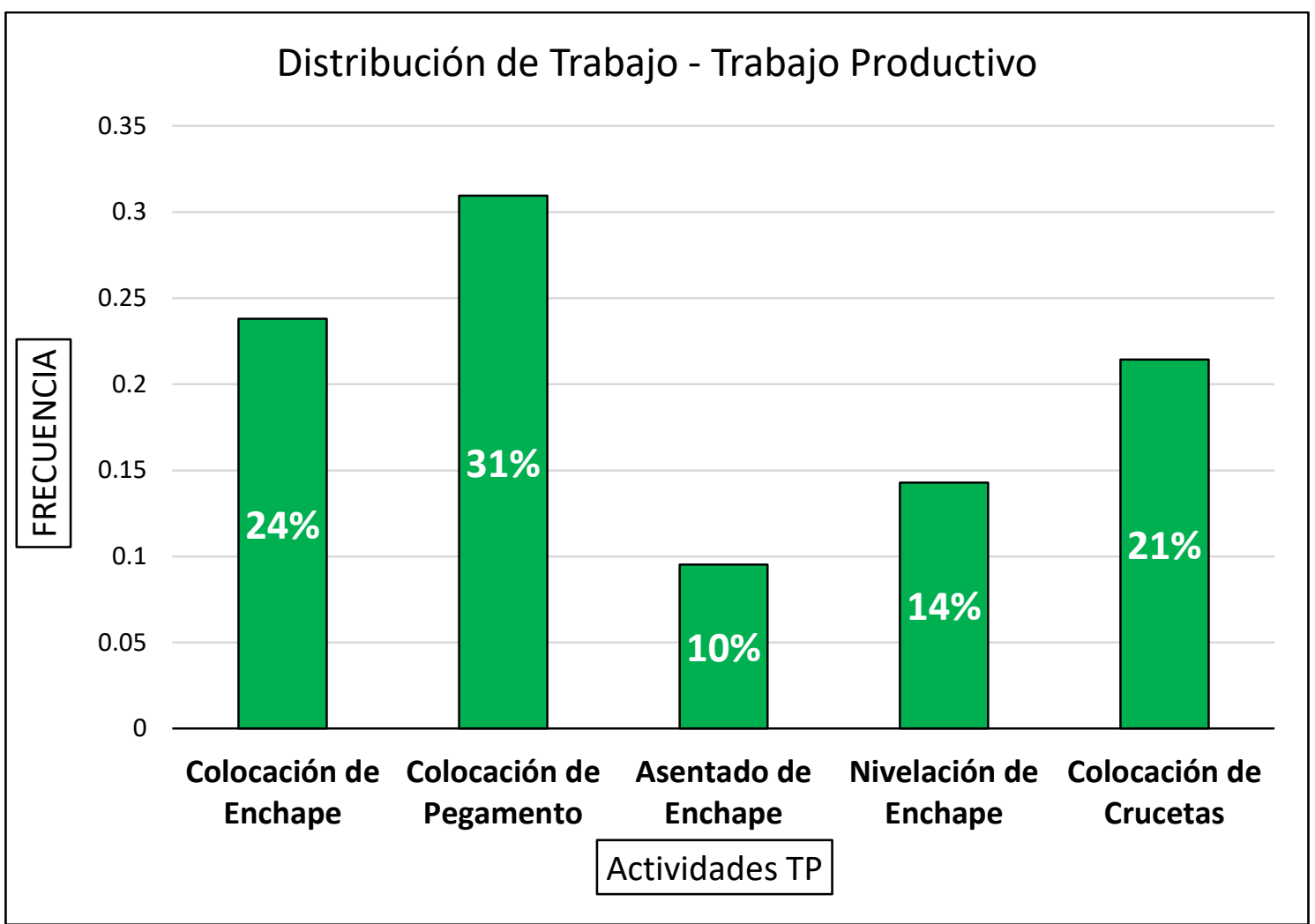

Fuente: Elaboración Propia. 
Figura $N^{\circ} 125$ : Distribución de Trabajo Contributorio - Carta Balance ${ }^{\circ} 19$

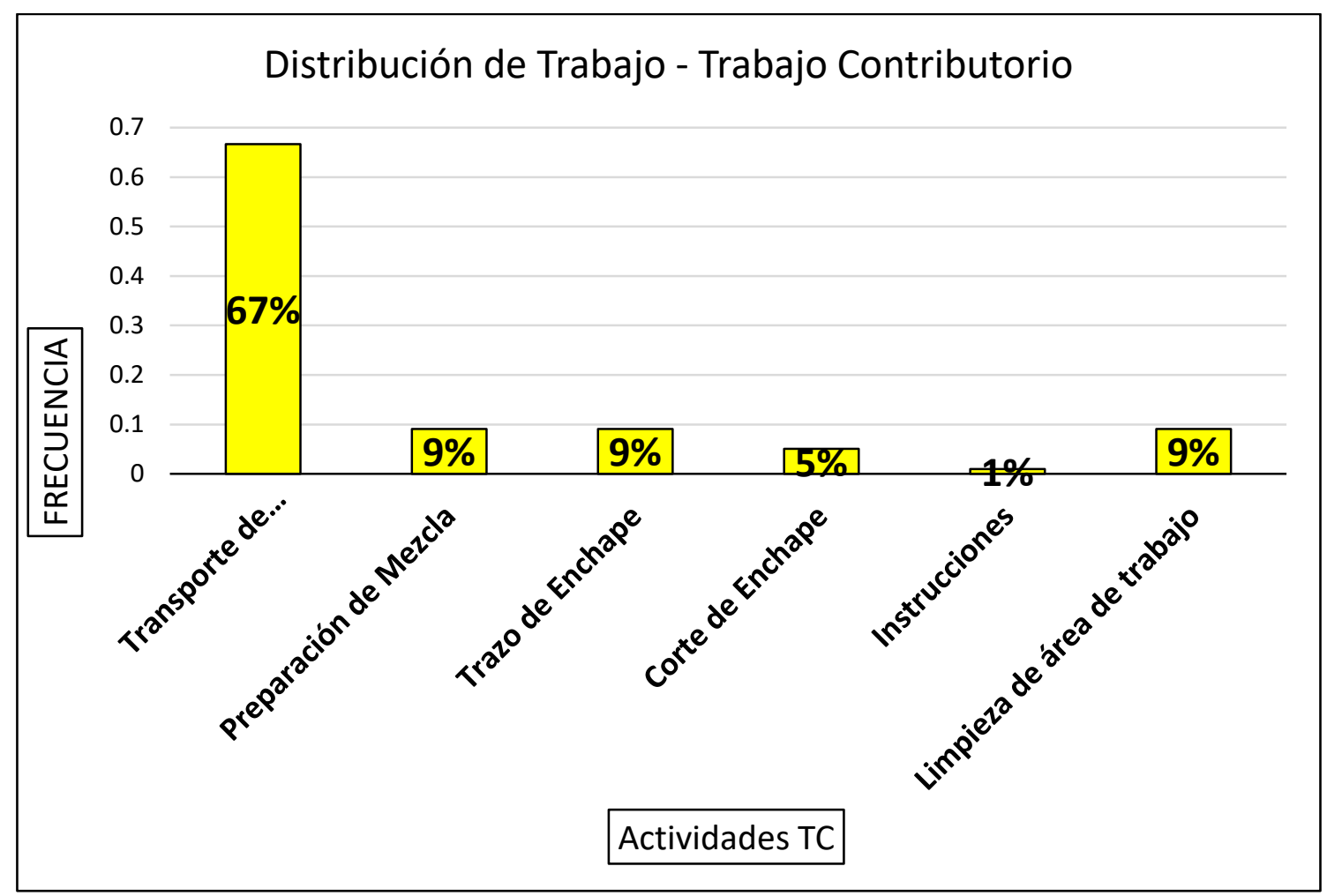

Fuente: Elaboración Propia.

Figura N ${ }^{\circ}$ 126: Distribución de Trabajo No Contributorio - Carta Balance ${ }^{\circ} 19$

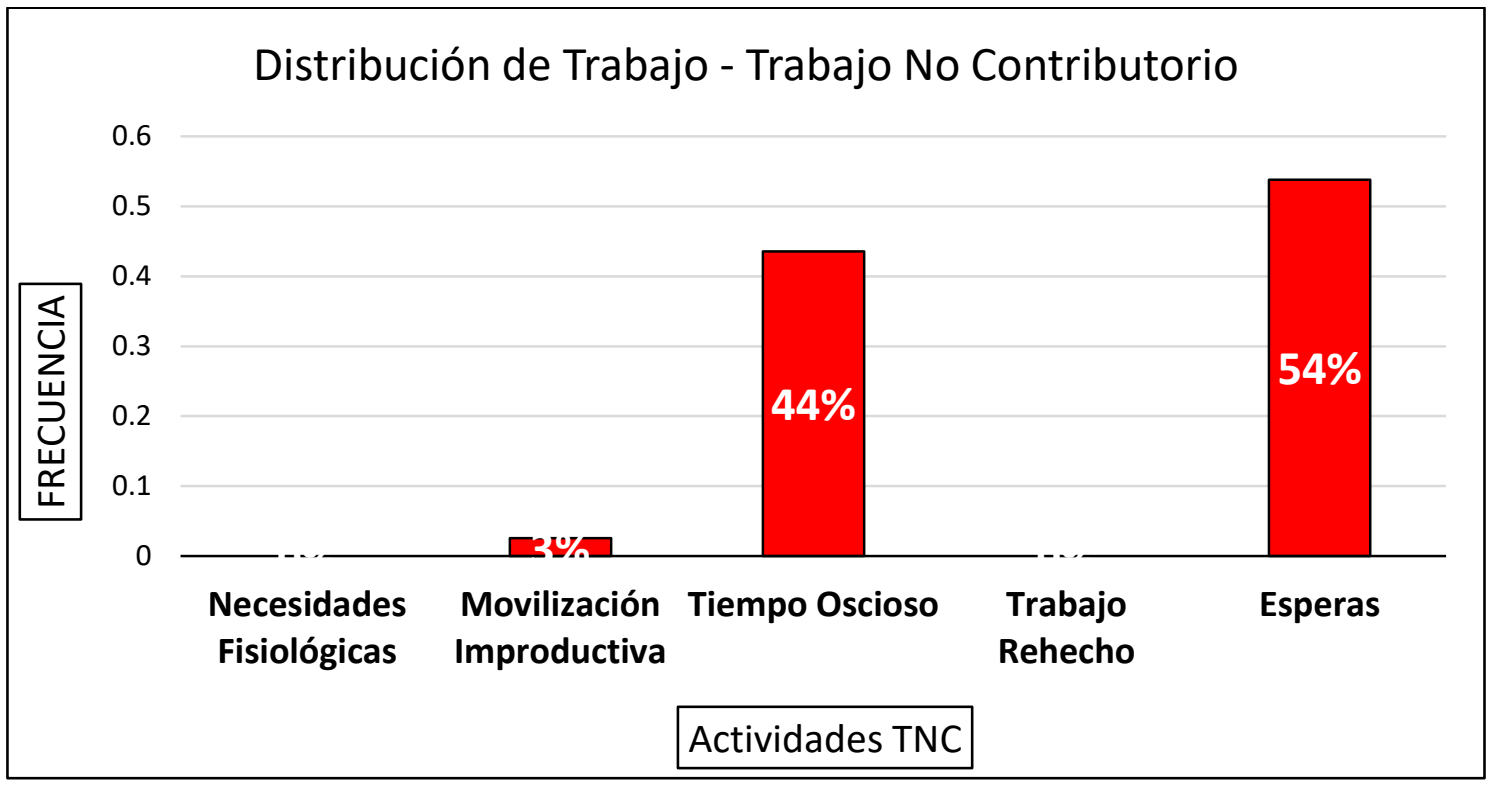

Fuente: Elaboración Propia. 
Tabla Nº 35: Carta Balance ²0 - Enchape de Cerámico

\begin{tabular}{|c|c|c|c|c|c|c|c|c|}
\hline $\mathrm{N}^{*}$ & Obrero \#1 & Obrero $\# 2$ & 31 & $\mathrm{CE}$ & $E$ & 61 & $A P$ & $\mathrm{TM}$ \\
\hline 1 & $\mathrm{CC}$ & E & 32 & $\mathrm{CP}$ & $E$ & 62 & AP & $\mathrm{TM}$ \\
\hline 2 & NE & E & 33 & NE & $E$ & 63 & NE & $\mathrm{TM}$ \\
\hline 3 & $\mathrm{NE}$ & TO & 34 & $\mathrm{CC}$ & $E$ & 64 & $C P$ & $\mathrm{TM}$ \\
\hline 4 & $C P$ & TO & 35 & AP & $E$ & 65 & $C P$ & TM \\
\hline 5 & $C P$ & TO & 36 & $A P$ & E & 66 & $C C$ & $\mathrm{TM}$ \\
\hline 6 & CE & TO & 37 & $\mathrm{CP}$ & $E$ & 67 & $C P$ & $\mathrm{TM}$ \\
\hline 7 & $C P$ & TO & 38 & $\mathrm{CC}$ & $E$ & 68 & CE & $\mathrm{TM}$ \\
\hline 8 & $C C$ & TO & 39 & $\mathrm{CC}$ & $E$ & 69 & $C \mathrm{C}$ & TM \\
\hline 9 & $\mathrm{CC}$ & TO & 40 & $C P$ & $E$ & 70 & $C C$ & $\mathrm{TM}$ \\
\hline 10 & COE & TO & 41 & NE & $E$ & 71 & NE & TM \\
\hline 11 & $\mathrm{CC}$ & TO & 42 & $C P$ & $E$ & 72 & TO & $\mathrm{TM}$ \\
\hline 12 & L & TO & 43 & COE & TM & 73 & AP & TM \\
\hline 13 & $B M$ & TO & 44 & $\mathrm{CE}$ & $\mathrm{TM}$ & 74 & $C C$ & $\mathrm{TM}$ \\
\hline 14 & $B M$ & TO & 45 & $\mathrm{CC}$ & $\mathrm{TM}$ & 75 & $\mathrm{CE}$ & $\mathrm{TM}$ \\
\hline 15 & $B M$ & TO & 46 & NE & $\mathrm{TM}$ & 76 & $C P$ & $\mathrm{TM}$ \\
\hline 16 & L & TO & 47 & NE & $\mathrm{TM}$ & 7 & CE & TM \\
\hline 17 & L & TO & 48 & $C C$ & $\mathrm{TM}$ & 78 & $C P$ & TM \\
\hline 18 & L & TO & 49 & $\mathrm{CP}$ & $\mathrm{TM}$ & 79 & $\triangle C$ & $\mathrm{TM}$ \\
\hline 19 & $C P$ & TO & 50 & $C P$ & $\mathrm{TM}$ & 80 & $C P$ & $\mathrm{TM}$ \\
\hline 20 & $C P$ & TO & 51 & $\mathrm{CE}$ & $\mathrm{TM}$ & 81 & NE & $\mathrm{TM}$ \\
\hline 21 & $C C$ & $\mathrm{TM}$ & 52 & $C P$ & $\mathrm{TM}$ & 82 & $C P$ & $\mathrm{TM}$ \\
\hline 22 & $A P$ & $\mathrm{TM}$ & 53 & NE & $\mathrm{TM}$ & 83 & NE & $\mathrm{TM}$ \\
\hline 23 & CE & TM & 54 & AP & TM & 84 & $\triangle C$ & TM \\
\hline 24 & $\mathrm{CP}$ & TM & 55 & NE & TM & 85 & $\triangle C$ & $\mathrm{TM}$ \\
\hline 25 & $C C$ & $E$ & 56 & $\mathrm{CP}$ & $\mathrm{TM}$ & 86 & $\mathrm{CE}$ & $\mathrm{TM}$ \\
\hline 26 & $C P$ & $E$ & 57 & NE & $\mathrm{TM}$ & 87 & NE & $\mathrm{TM}$ \\
\hline 27 & $A P$ & $E$ & 58 & $t$ & $\mathrm{TM}$ & 88 & TO & $E$ \\
\hline 28 & $C P$ & E & 59 & CE & $\mathrm{TM}$ & 89 & TO & E \\
\hline 29 & NE & $E$ & 60 & NE & $\mathrm{TM}$ & 90 & TM & E \\
\hline 30 & $\mathrm{CP}$ & E & & & & & & \\
\hline
\end{tabular}

Fuente: Elaboración Propia. 
Figura Nº 127: Distribución de Trabajo de Enchape de Cerámico - Carta Balance ²0

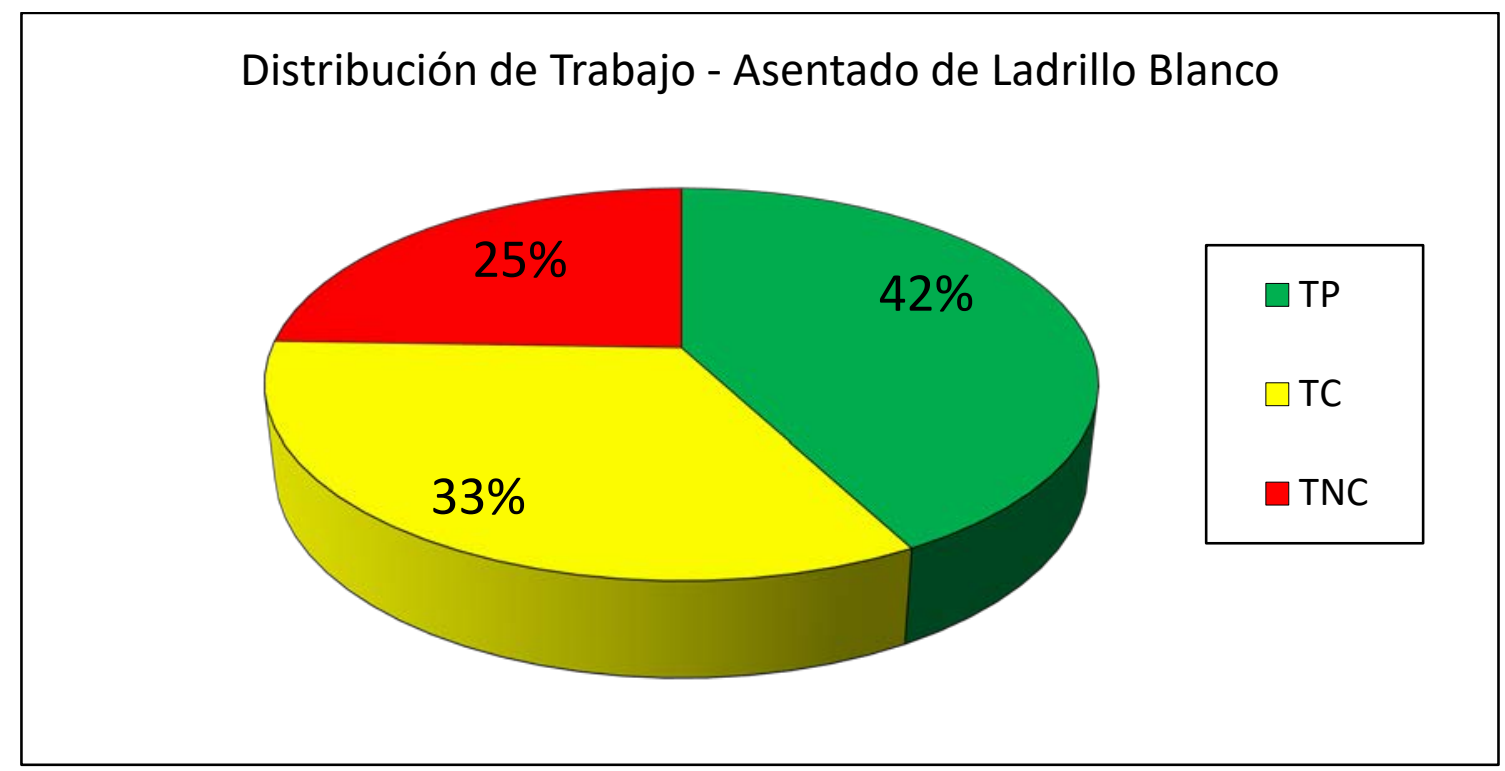

Fuente: Elaboración Propia.

Figura No 128: Distribución de Trabajo Productivo - Carta Balance ${ }^{\circ} 20$

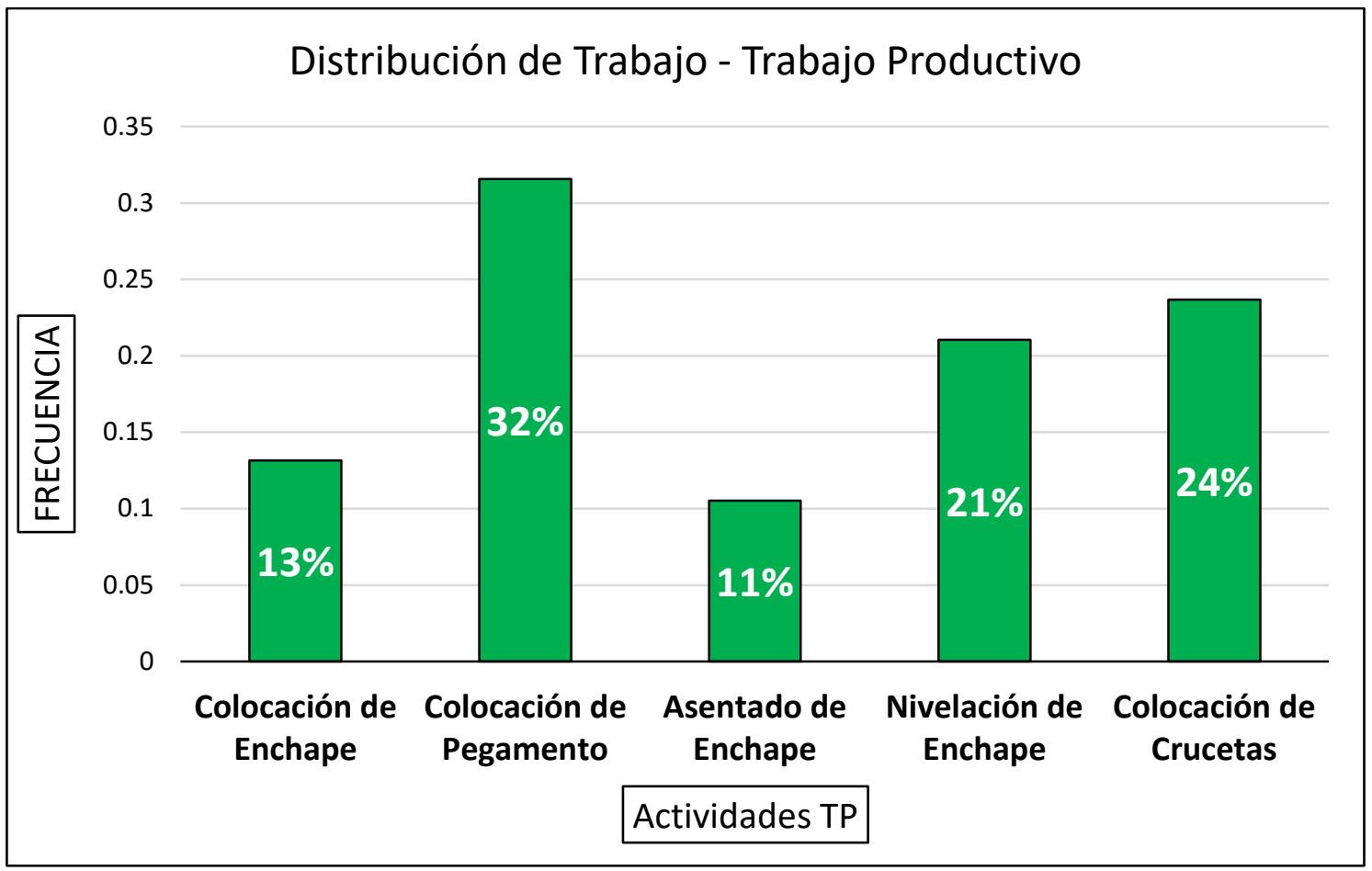

Fuente: Elaboración Propia. 
Figura $N^{\circ}$ 129: Distribución de Trabajo Contributorio - Carta Balance ${ }^{\circ} 20$

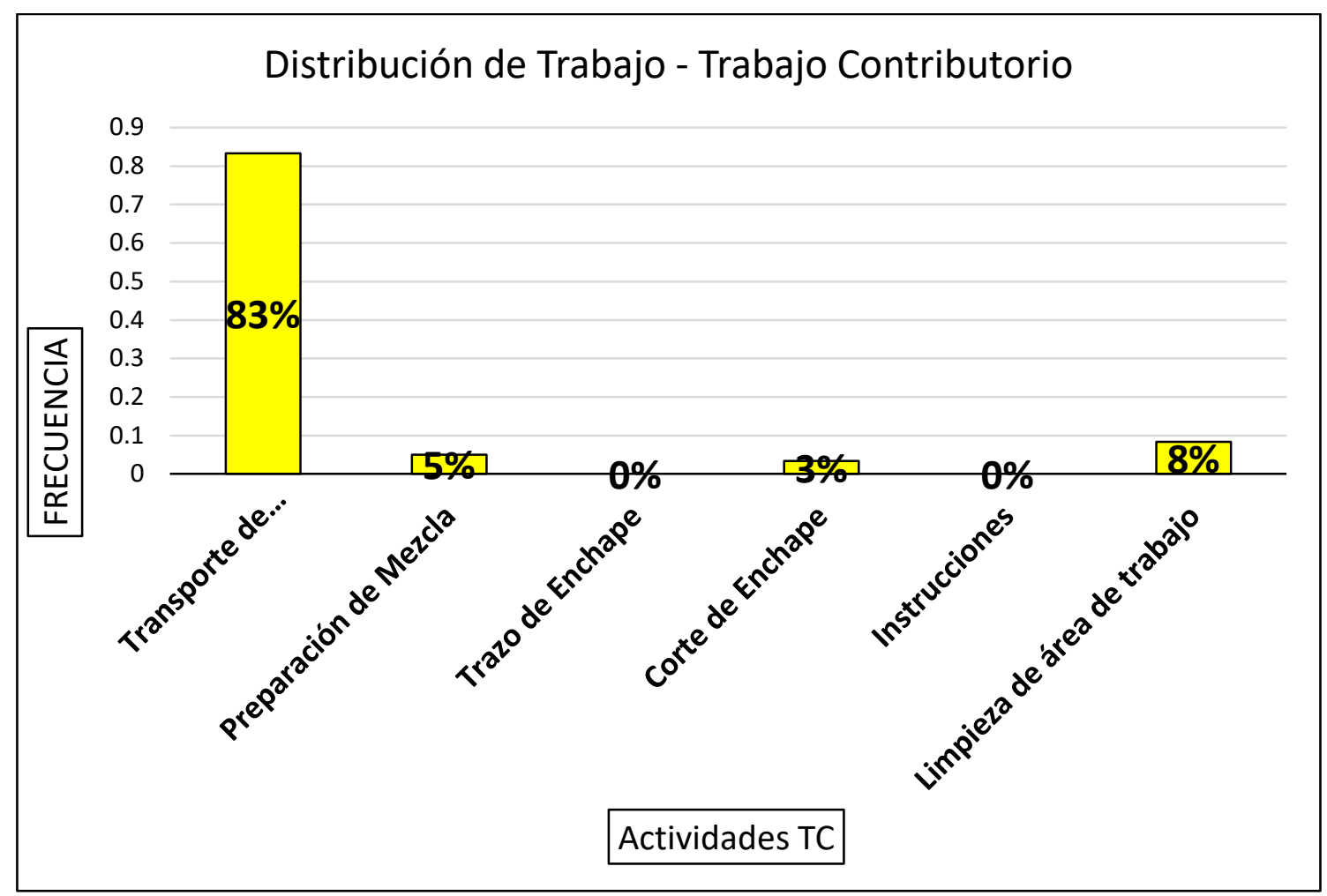

Fuente: Elaboración Propia.

Figura No 130: Distribución de Trabajo No Contributorio - Carta Balance ${ }^{\circ} 20$

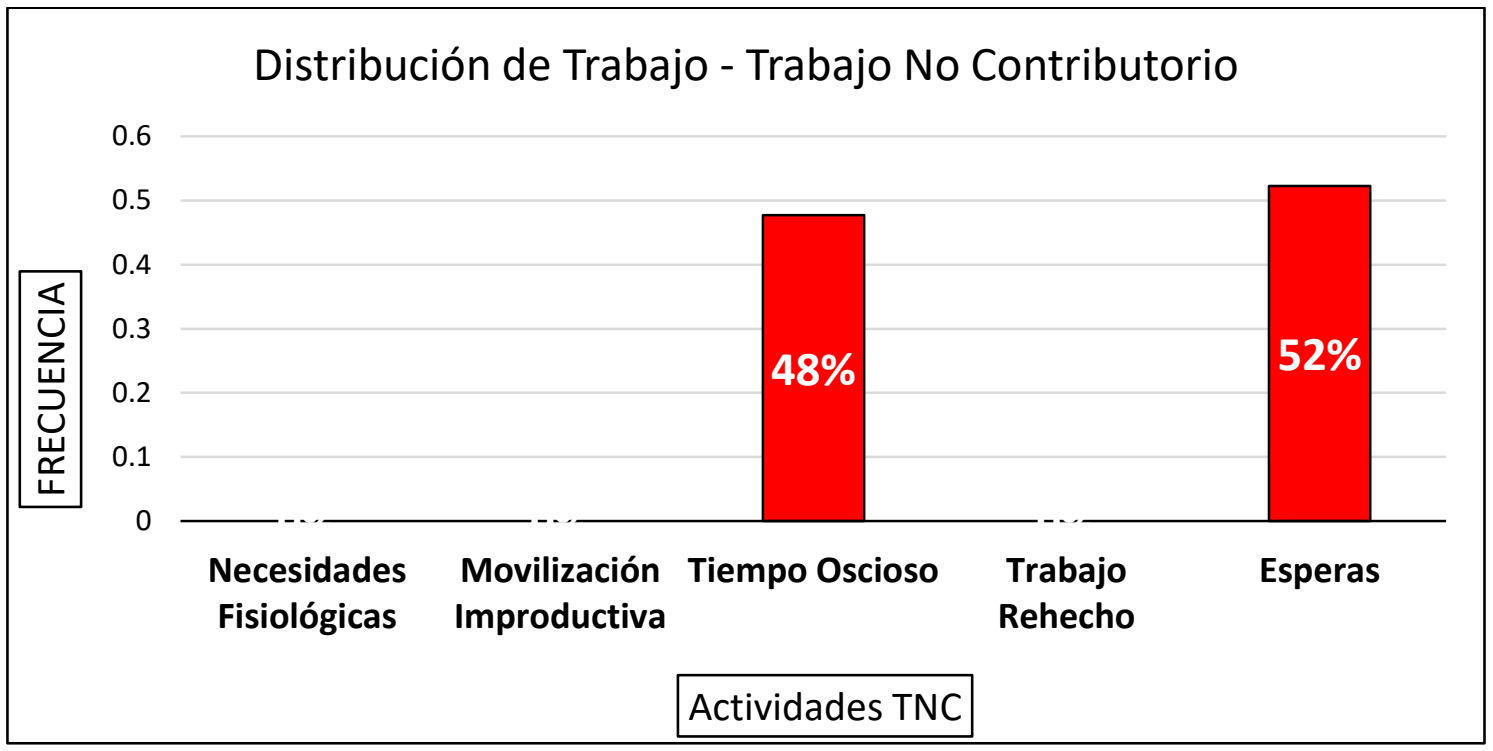

Fuente: Elaboración Propia. 
Tabla N 36: Carta Balance ${ }^{\circ} 21$ - Enchape de Cerámico

\begin{tabular}{|c|c|c|c|c|c|c|c|c|}
\hline $\mathrm{N}^{*}$ & Obrero \#1 & Obrero \#2 & 31 & $\mathrm{CC}$ & $\mathrm{TM}$ & 61 & 1 & TM \\
\hline 1 & $\mathrm{CC}$ & $\mathrm{E}$ & 32 & NE & $\mathrm{TM}$ & 62 & $\mathrm{TM}$ & TM \\
\hline 2 & $C P$ & E & 33 & $\mathrm{CE}$ & $\mathrm{TM}$ & 63 & 1 & $\mathrm{TM}$ \\
\hline 3 & $\mathrm{CC}$ & $E$ & 34 & $\mathrm{CC}$ & $\mathrm{TM}$ & 64 & 1 & TM \\
\hline 4 & $\mathrm{CC}$ & E & 35 & L & $\mathrm{TM}$ & 65 & 1 & TM \\
\hline 5 & CE & E & 36 & $C P$ & $\mathrm{TM}$ & 66 & 1 & E \\
\hline 6 & $C P$ & E & 37 & $C P$ & $\mathrm{TM}$ & 67 & $\mathrm{TM}$ & E \\
\hline 7 & $C P$ & E & 38 & $\mathrm{CP}$ & $\mathrm{TM}$ & 68 & $\mathrm{TM}$ & E \\
\hline 8 & $C P$ & $E$ & 39 & CE & $\mathrm{TM}$ & 69 & $\mathrm{TM}$ & $E$ \\
\hline 9 & $C P$ & $E$ & 40 & $C C$ & $\mathrm{TM}$ & 70 & $\mathrm{TM}$ & $E$ \\
\hline 10 & BM & E & 41 & NE & $\mathrm{TM}$ & 71 & $\mathrm{TM}$ & E \\
\hline 11 & BM & E & 42 & NE & E & 72 & $B M$ & E \\
\hline 12 & BM & E & 43 & NE & $E$ & 73 & $B M$ & $\mathrm{E}$ \\
\hline 13 & $C P$ & $E$ & 44 & $\mathrm{TM}$ & $E$ & 74 & BM & $E$ \\
\hline 14 & CE & E & 45 & $\mathrm{CE}$ & $E$ & 75 & $\mathrm{TM}$ & $\mathrm{E}$ \\
\hline 15 & NE & $E$ & 46 & $\mathrm{CC}$ & $E$ & 76 & L & $E$ \\
\hline 16 & NE & $\mathrm{TM}$ & 47 & $\mathrm{CE}$ & $E$ & 77 & $C P$ & $\mathrm{E}$ \\
\hline 17 & $\mathrm{CC}$ & TM & 48 & CE & $\mathrm{TM}$ & 78 & CE & E \\
\hline 18 & $\mathrm{CC}$ & $\mathrm{TM}$ & 49 & $\mathrm{CP}$ & $\mathrm{TM}$ & 79 & $\mathrm{CC}$ & $E$ \\
\hline 19 & CE & $\mathrm{TM}$ & 50 & CE & $\mathrm{TM}$ & 80 & CE & $E$ \\
\hline 20 & CE & TM & S1 & $\mathrm{CE}$ & $\mathrm{TM}$ & 81 & $C E$ & $E$ \\
\hline 21 & $C P$ & TM & 52 & NE & $E$ & 82 & TE & E \\
\hline 22 & $\mathrm{NE}$ & $\mathrm{TM}$ & 53 & $\mathrm{CE}$ & $E$ & 83 & $\mathrm{CE}$ & $\mathrm{E}$ \\
\hline 23 & $\mathrm{CC}$ & TM & 54 & NE & $\mathrm{TM}$ & 84 & CE & E \\
\hline 24 & $\mathrm{NE}$ & $\mathrm{TM}$ & 55 & $\mathrm{CE}$ & $\mathrm{TM}$ & 85 & $C P$ & $E$ \\
\hline 25 & CE & TM & 56 & CE & $\mathrm{TM}$ & 86 & CE & $\mathrm{E}$ \\
\hline 26 & $C P$ & $\mathrm{TM}$ & 57 & $\mathrm{CP}$ & $\mathrm{TM}$ & 87 & COE & $E$ \\
\hline 27 & $C P$ & $\mathrm{TM}$ & 58 & CE & $\mathrm{TM}$ & 88 & $\mathrm{CC}$ & $E$ \\
\hline 28 & $\mathrm{NE}$ & $\mathrm{TM}$ & 59 & $\mathrm{TM}$ & $\mathrm{TM}$ & 89 & $C P$ & $E$ \\
\hline 29 & $\mathrm{NE}$ & $\mathrm{TM}$ & 60 & $\mathrm{TM}$ & $\mathrm{TM}$ & 90 & $\mathrm{CC}$ & $\mathrm{TM}$ \\
\hline 30 & $C C$ & TM & & & & & & \\
\hline
\end{tabular}

Fuente: Elaboración Propia. 
Figura Nº 131: Distribución de Trabajo de Enchape de Cerámico - Carta Balance ${ }^{\circ} 21$

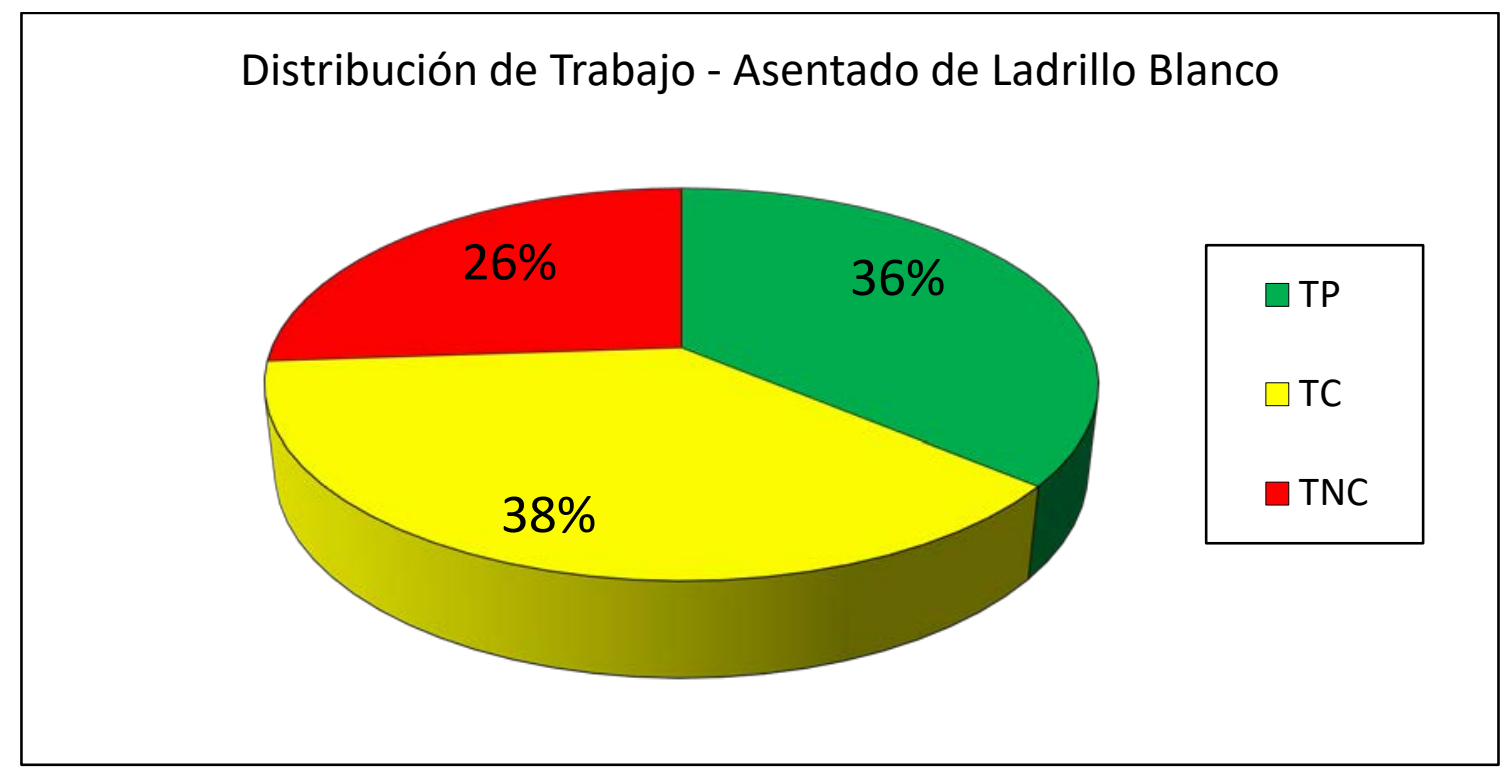

Fuente: Elaboración Propia.

Figura No 132: Distribución de Trabajo Productivo - Carta Balance ${ }^{\circ} 21$

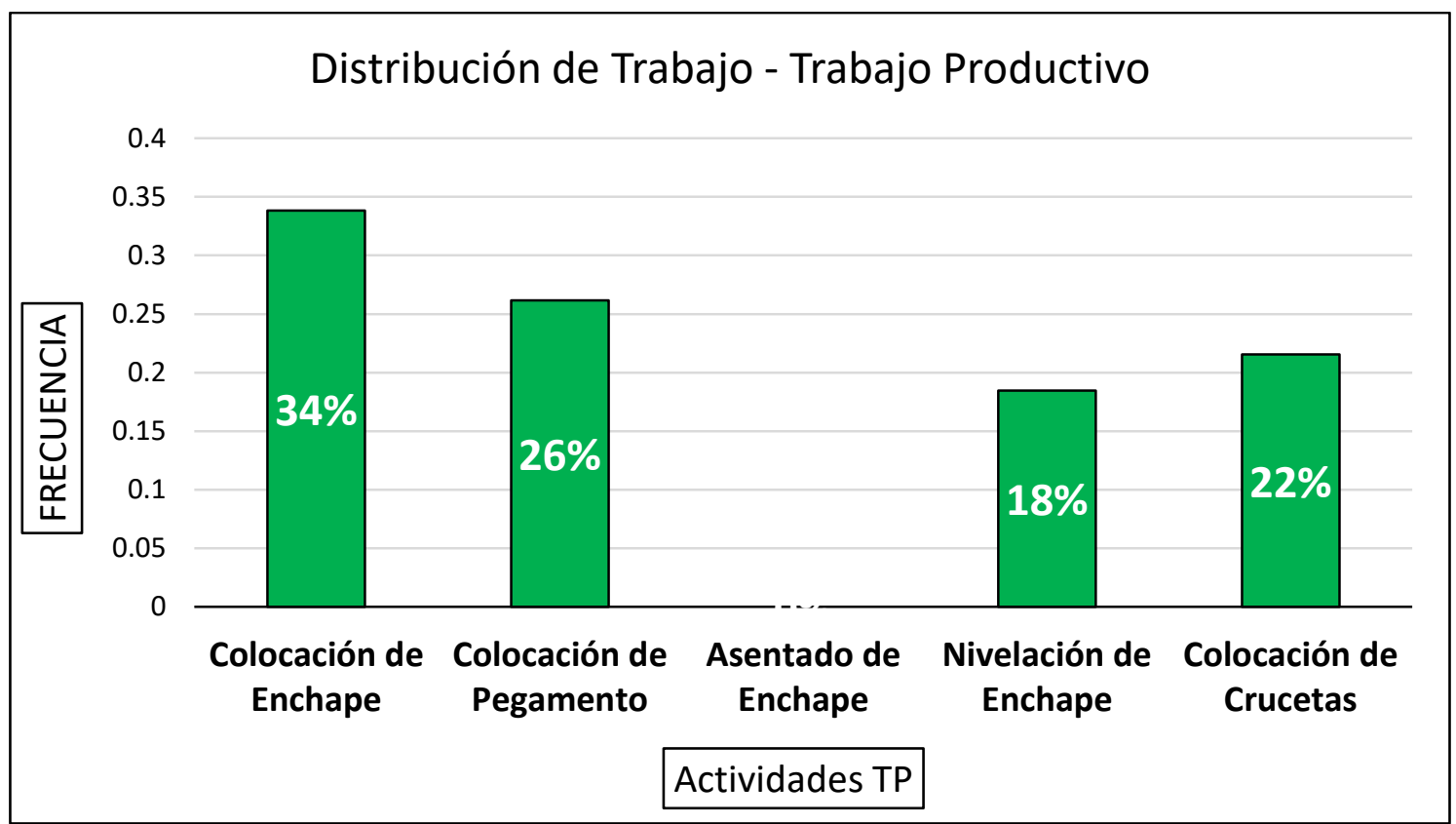

Fuente: Elaboración Propia. 
Figura $N^{\circ}$ 133: Distribución de Trabajo Contributorio - Carta Balance ${ }^{\circ} 21$

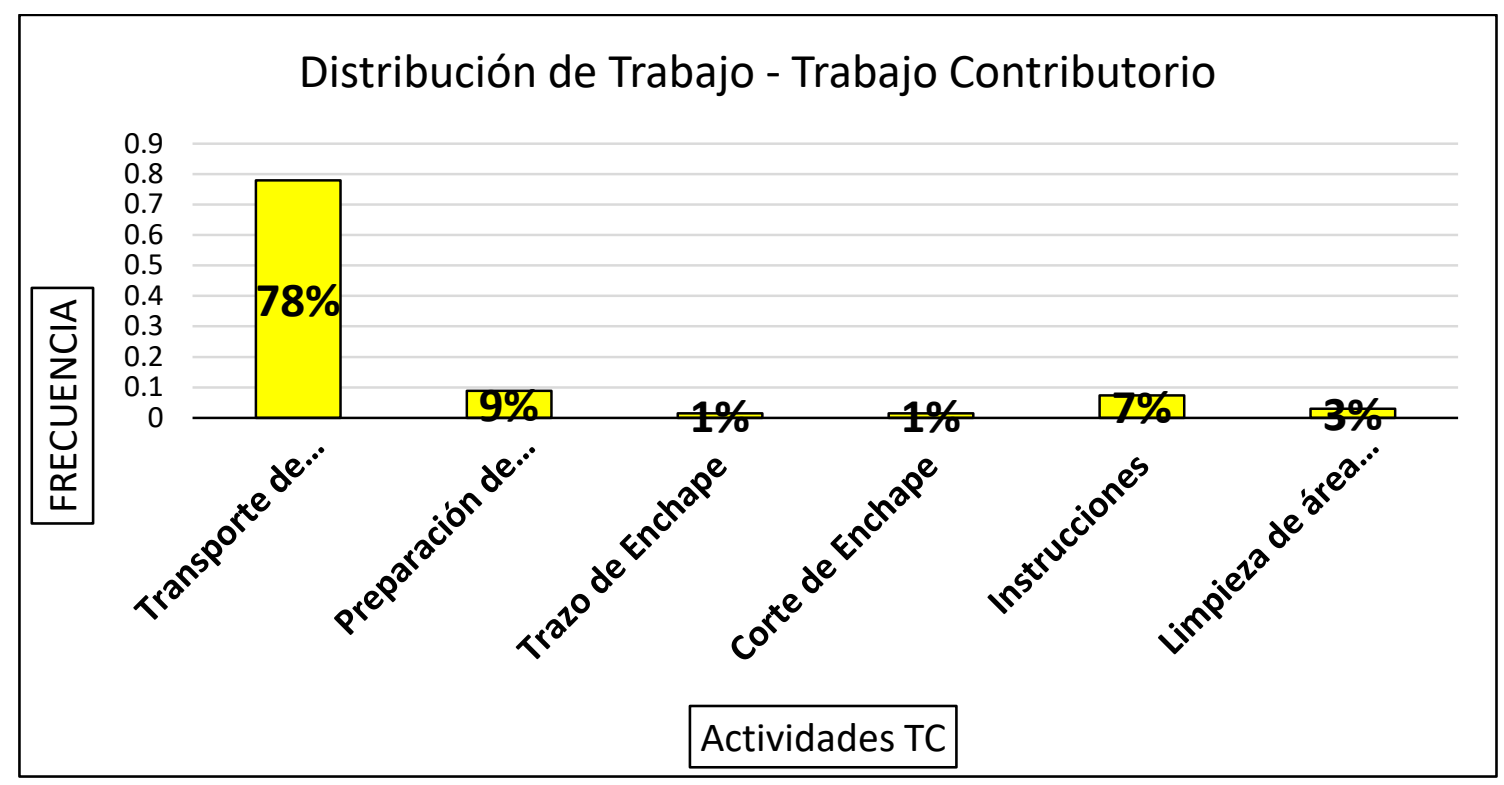

Fuente: Elaboración Propia.

Figura N ${ }^{\circ}$ 134: Distribución de Trabajo No Contributorio - Carta Balance ${ }^{\circ} 21$

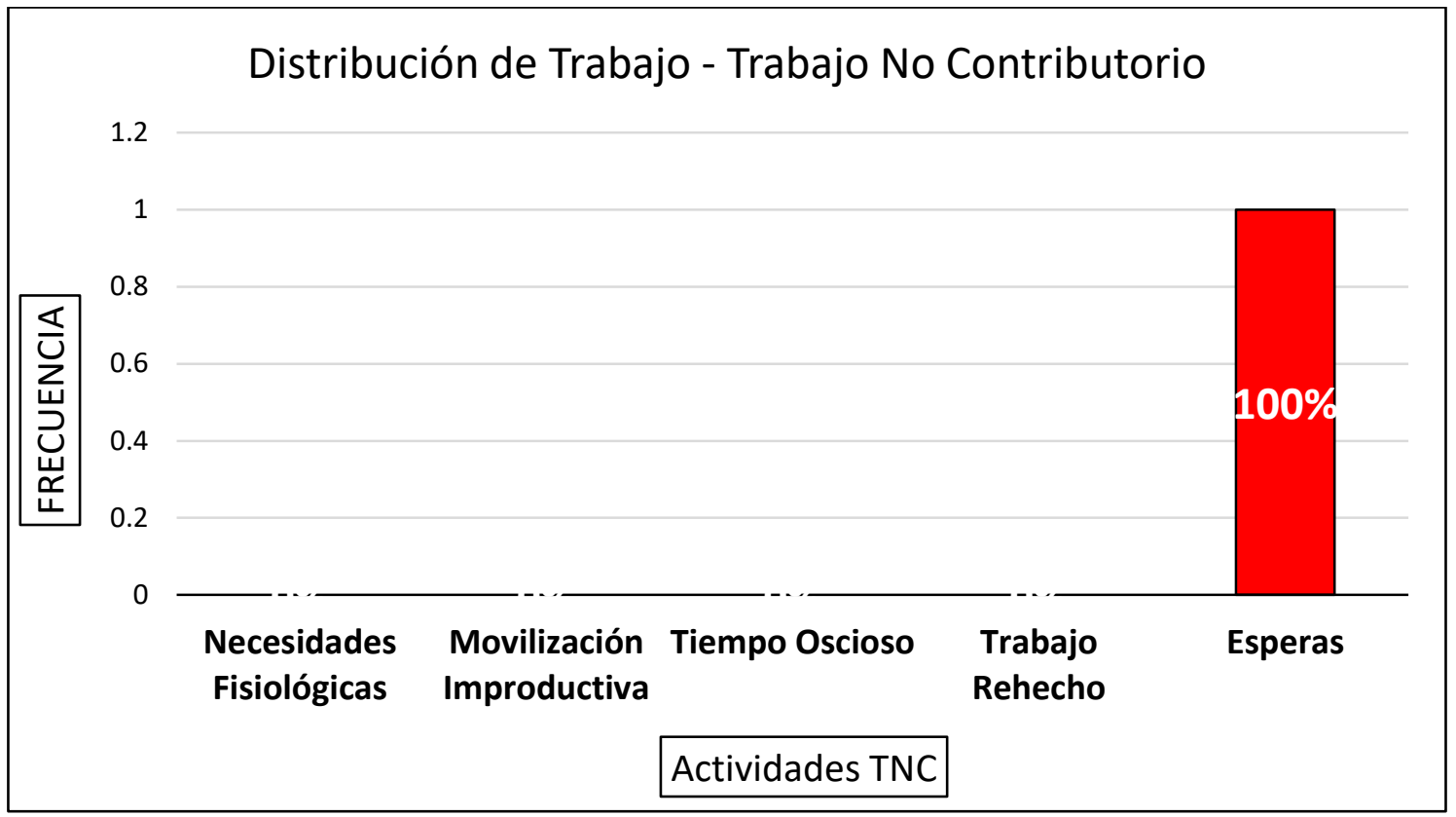

Fuente: Elaboración Propia. 